Supporting Information

\title{
Synthesis of the Antimalarial Peptide Aldehyde, a Precursor of Kozupeptin A, Utilizing a Designed Hydrophobic Anchor Molecule
}

Yumi Hayashi, ${ }^{a}$ Tomoyasu Hirose, ${ }^{a, b}$ Masato Iwatsuki, ${ }^{a, b}$ Satoshi Ōmura, a,b and Toshiaki Sunazuka*,a,b

${ }^{a}$ Graduate School of Infection Control Sciences, Kitasato University, 5-9-1 Shirokane, Minato-ku, Tokyo 108-8641, Japan

bKitasato Institute for Life Sciences, Kitasato University, 5-9-1 Shirokane, Minato-ku, Tokyo 108-8641, Japan 


\section{Table of contents}

$\begin{array}{ll}\text { Table of contents } & \text { p. } 2\end{array}$

$\begin{array}{ll}\text { General remarks } & \text { p. } 3\end{array}$

$\begin{array}{lll}\text { Screening of condensation reagents to suppress the epimerization in our previous report } & \text { p. } 3\end{array}$

$\begin{array}{ll}\text { Experimental procedures and compounds characterization } & \text { p. } 4\end{array}$

$\begin{array}{ll}\text { Preparation of TAGa-type anchor molecule } & \text { p. } 4\end{array}$

$\begin{array}{ll}\text { Preparation of TAGb-type anchor molecule } & \text { p. } 6\end{array}$

$\begin{array}{lr}\text { Peptide elongations (using TAGa-type anchor molecule) } & \text { p. } 7\end{array}$

$\begin{array}{ll}\text { Reduction to afford the aldehyde } & \text { p. } 10\end{array}$

Use of a model substrate p. 12

$\begin{array}{ll}\text { Using TAGb-type anchor molecule } & \text { p. } 14\end{array}$

$\begin{array}{ll}\text { Selective deprotection of TAG benzyl group under acid conditions and reduction to the aldehydes } & \text { p. } 17\end{array}$

$\begin{array}{ll}\text { References } & \text { p. } 18\end{array}$

$\begin{array}{ll}\text { NMR spectra of the products } & \text { p. } 19\end{array}$ 


\section{General remarks}

Infrared (IR) spectra were recorded on a Horiba FT-210 spectrometer. UV spectra were measured with a Beckman DU640 spectrophotometer. NMR spectra were measured on a JEOL JNM-ECA-500 spectrometer with ${ }^{1} \mathrm{H}$ NMR at $500 \mathrm{MHz}$ and ${ }^{13} \mathrm{C}$ NMR at $125 \mathrm{MHz}$. Chemical shifts were reported in ppm from the internal solvent peaks for chloroform- $d_{1}\left(\mathrm{CDCl}_{3}\right)\left({ }^{1} \mathrm{H} ; \delta=7.26 \mathrm{ppm},{ }^{13} \mathrm{C} ; \delta=\right.$ $77.16 \mathrm{ppm})$ or dimethylsulfoxide- $d_{6}\left(\left(\mathrm{CD}_{3}\right)_{2} \mathrm{SO}\right.$, DMSO- $\left.d_{6}\right)\left({ }^{1} \mathrm{H} ; \delta=2.50 \mathrm{ppm},{ }^{13} \mathrm{C} ; \delta=39.52 \mathrm{ppm}\right) .{ }^{1} \mathrm{H}$ NMR data were reported as follows: chemical shift (integration, multiplicity $(s=$ singlet, $d=$ doublet, $t=$ triplet, $q=$ quartet, $m=$ multiplet, $b r=b r o a d, a p p=$ apparent), coupling constants $(\mathrm{Hz})$ ). Liquid chromatography-electrospray ionization mass spectrometry (LC/MS) was performed on a Waters AQUITY UPLC H-Class using acetonitrile ( $\mathrm{MeCN}$ ) solvent system containing $0.05 \%$ formic acid. The high-resolution mass spectra (HRMS) were performed on a JEOL JMS-AX505 HA, a JEOL JMS-700 MStation, or a JEOL JMS-T100LP. Optical rotations were measured on a JASCO P-1010 polarimeter.

For thin layer chromatography (TLC) analysis, Merck precoated TLC plates (silica gel $60 \mathrm{GF}_{254}, 0.25 \mathrm{~mm}$ ) were used. Flash chromatography was carried out with Kanto Chemical silica gel (silica gel 60N, spherical neutral, 0.040-0.050 mm) or Fuji Silysia silica gel (FL60D, avg. $0.060 \mathrm{~mm}$ ). For purification with preparative thin layer chromatography (PLC), Merck precoated PLC plates (silica gel $60 \mathrm{GF}_{254}, 0.5 \mathrm{~mm}$ ) were used.

Unless otherwise noted, reagents and solvents were commercially available and used without further purification. In experiments requiring dry solvents, dichloromethane (DCM) and tetrahydrofuran (THF) were purchased from Kanto Chemical Co. Inc. as "Dehydrated." For Fmoc-protected amino acids, Fmoc-Ala-OH, Fmoc-Asn-OH, Fmoc-Val-OH, and Fmoc-Thr-OH· $\mathrm{H}_{2} \mathrm{O}$ were purchased from Watanabe Chemical Industries, Ltd. Fmoc- $(2 S, 4 R)-4-\mathrm{MePro}-\mathrm{OH}$ was prepared by the procedure described in the literature. ${ }^{[1]}$

\section{Screening of condensation reagents to suppress the epimerization in our previous report $^{[2]}$ (not shown in the previous paper)}

\section{Experimental procedure and determination of the stereochemistry of $\alpha$-position of Ala unit (L or D)}

To a solution of $\mathbf{S} 1(5.0 \mathrm{mg}, 0.01 \mathrm{mmol}, 1.0 \mathrm{eq}$.), $\mathrm{MeO}(\mathrm{Me}) \mathrm{NH} \cdot \mathrm{HCl}$, and condensation reagent in dimethylformamide (DMF) $(0.036 \mathrm{M})$ was added $\mathrm{N}, \mathrm{N}$-diisopropylethylamine (DIPEA) at room temperature. After stirring at room temperature until $\mathbf{S 1}$ was completely consumed, the reaction mixture was then treated with aqueous $1 \mathrm{~N} \mathrm{HCl}$ in an ice bath to quench the excess amine reagent, poured into a separatory funnel containing ethyl acetate (EtOAc) $(20 \mathrm{~mL})$, and washed with aqueous $1 \mathrm{~N} \mathrm{HCl}(2 \times 10 \mathrm{~mL})$ and sat. $\mathrm{NaCl}$ aq. $(10 \mathrm{~mL})$. The organic layer was dried over $\mathrm{Na}_{2} \mathrm{SO}_{4}$, filtered, and concentrated in vacuo to afford the crude product S2.

The diastereoselective ratio (L/D) of $\alpha$-position of Ala unit was determined by advanced Marfey's analysis. ${ }^{[3]} \mathrm{A}$ sample (100 $\left.\mu \mathrm{g}\right)$ of the above crude product $\mathbf{S 2}$ was dissolved in $6 \mathrm{~N} \mathrm{HCl}(500 \mu \mathrm{L})$ and heated for $3 \mathrm{~h}$ at $100{ }^{\circ} \mathrm{C}$. After cooling to room temperature, the hydrolysate was evaporated to dryness in vacuo, and the residue was dissolved in $100 \mu \mathrm{L}$ of water. $50 \mu \mathrm{L}$ of the hydrolysate aq. was treated with $25 \mu \mathrm{L}$ of $1 \mathrm{M} \mathrm{NaHCO}_{3}$ and $50 \mu \mathrm{L}$ of $1 \%$ 1-fluoro-2,4-dinitrophenyl-5-D-leucinamide (D-FDLA) in acetone. The mixture was heated for $1 \mathrm{~h}$ at $37^{\circ} \mathrm{C}$. After cooling to room temperature, the reaction mixture was neutralized with $1 \mathrm{~N} \mathrm{HCl}$, and concentrated in vacuo. The residue was dissolved with $200 \mu \mathrm{L}$ of $\mathrm{MeCN}$, filtered to remove salt and analyzed by UPLC-MS (Waters Co., USA) on reversed-phase column (BEH C18 column; $2.1 \times 50 \mathrm{~mm}, 1.7 \mu \mathrm{m}, 0.5 \mathrm{~mL} / \mathrm{min}$ ) with a linear gradient from $50 \%$ to $100 \%$ aqueous MeCN containing formic acid (mobile phase A; $100 \% \mathrm{MeCN}+0.05 \%$ formic acid and mobile phase $\mathrm{B} ; 90 \% \mathrm{H} \mathrm{H}_{2} \mathrm{O} 10 \% \mathrm{MeCN}+$ $0.05 \%$ formic acid) for $10 \mathrm{~min}$.

Commercial L-Ala-OH and D-Ala-OH standards were adjusted to $1 \mathrm{mM}$ with water and were subjected to advanced Marfey's analysis as described above. The ESI positive mode was used for the detection of L- or D-Ala-D-FDLA derivative, and the L/D ratio of a-position of Ala unit was determined by a comparison of retention time between the Ala-D-FDLA derivatives from the hydrolysate of crude product $\mathbf{S} 2$ and standards. The results were summarized in Table $\mathbf{S 1 .}$ 
Table S1. Screening of condensation reagents in the transformation of carboxylic acid S1 to Weinreb amide S2.

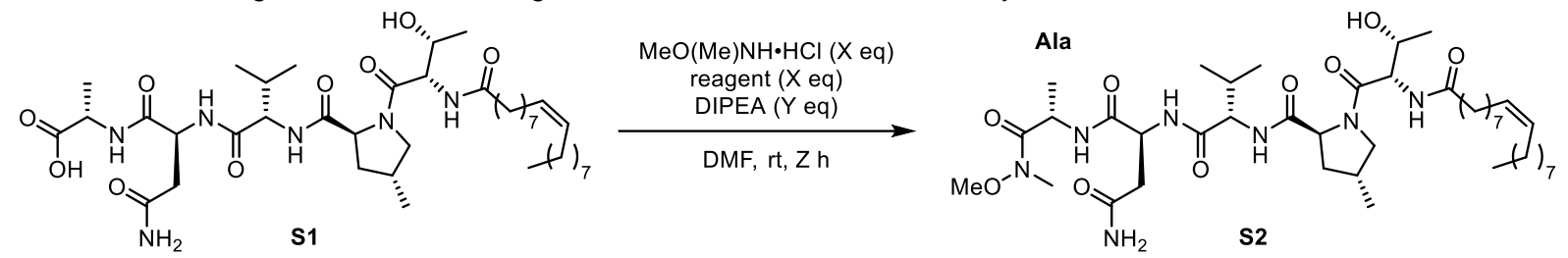

\begin{tabular}{ccccccc}
\hline entry & reagent & $\mathrm{X} \mathrm{eq}$ & $\mathrm{Y} \mathrm{eq}$ & $\mathrm{Z} \mathrm{h}^{[\mathrm{a}]}$ & L/D of Ala unit $^{[\mathrm{b}]}$ & yield (isolated) \\
\hline 1 & HBTU & 1.2 & 2.4 & 3 & $62 / 38$ & - \\
2 & HATU & 1.2 & 2.4 & 4.5 & $82 / 18$ & - \\
3 & COMU & 1.2 & 2.4 & 4.5 & $86 / 14$ & - \\
4 & PyBroP & 1.8 & 4.8 & 5 & $61 / 39$ & - \\
5 & PyAOP & 1.2 & 2.4 & 6 & $89 / 11$ & - \\
6 & DEPBT & 3.2 & 6.4 & 16 & $97 / 3$ & quant. $^{[\mathrm{c}]}$ \\
\hline
\end{tabular}

${ }^{[\mathrm{a}]}$ In all entries, full conv. ${ }^{[\mathrm{b}]}$ Determined by advanced Marfey's analysis after complete hydrolysis.

[c] $120 \mathrm{mg}$ scale of $\mathbf{S} 1(0.15 \mathrm{mmol})$. The crude was purified by flash column chromatography on silica gel $\left(\mathrm{CHCl}_{3} / \mathrm{MeOH}\right)$.<smiles>CN(C)C(On1nnc2ccccc21)=[N+](C)C</smiles>

HBTU<smiles>CN(C)C(On1nnc2cccnc21)N(C)C</smiles>

HATU<smiles>CCOC(=O)C(C#N)=NOC(N1CCOCC1)=[N+](C)C</smiles>

COMU

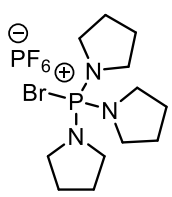

PyBroP

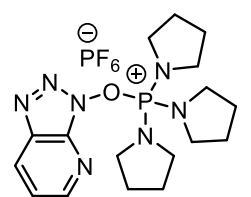

PyAOP<smiles>CCOP(=O)(OCC)On1nnc2ccccc2c1=O</smiles>

DEPBT

\section{Experimental procedures and compounds characterization}

\section{Preparation of TAGa-type anchor molecule}

Scheme S1. Synthesis of a new designed hydrophobic anchor molecule (TAGa-type anchor molecule)

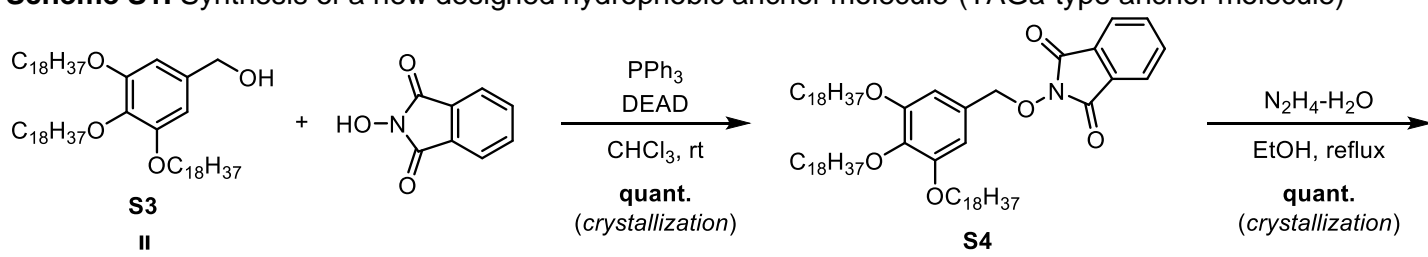

HO-TAGa

(20 g scale)

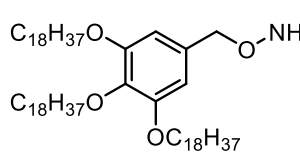

S5

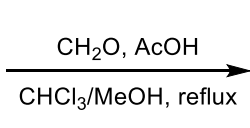

quant.

(crystallization)

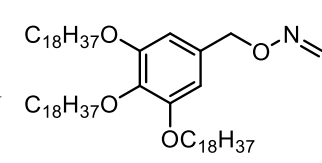

S6

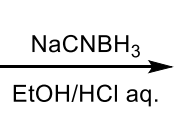

$83 \%$

(silica gel column)

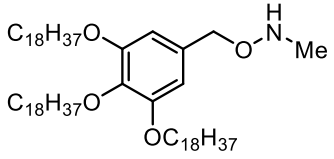

3

TAGa ${ }^{\text {II }}{ }^{-\mathrm{N}}$

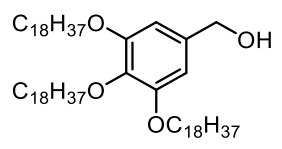

3,4,5-Tris(octadecyloxy)benzyl alcohol (S3) (HO-TAGa)

$\mathrm{HO}-\mathrm{TAGa}(\mathrm{S} 3)$ was prepared by the procedure described in the literature as a white powder. ${ }^{[4]} \mathrm{mp} 68-69{ }^{\circ} \mathrm{C} ;{ }^{1} \mathrm{H} \mathrm{NMR}(500 \mathrm{MHz}$, $\left.\mathrm{CDCl}_{3}\right) \delta 6.56(2 \mathrm{H}, \mathrm{s}), 4.59(2 \mathrm{H}, \mathrm{s}), 3.98-3.92(6 \mathrm{H}, \mathrm{m}$, overlapped), 1.82-1.71 (6H, m, overlapped), 1.50-1.43 (6H, br m, overlapped), 1.35-1.22 (84H, br m, overlapped), $0.88(9 \mathrm{H}, \mathrm{t}, J=7.0 \mathrm{~Hz}) ;{ }^{13} \mathrm{C} \mathrm{NMR}\left(125 \mathrm{MHz}, \mathrm{CDCl}_{3}\right) \delta 153.4,137.7,136.2,105.5,73.6,69.3$, 65.8, 32.1, 30.5, 29.9-29.5 (many signals overlapped), 26.29, 26.26, 22.8, 14.3; IR (KBr) $v\left(\mathrm{~cm}^{-1}\right) 3541,2916,2848,1594,1462$, 1439, 1118, 719; HRMS (FAB, NBA matrix) Calcd. for $\mathrm{C}_{61} \mathrm{H}_{116} \mathrm{O}_{4}: 912.8874\left([\mathrm{M}]^{+}\right)$, Found: 912.8876. 


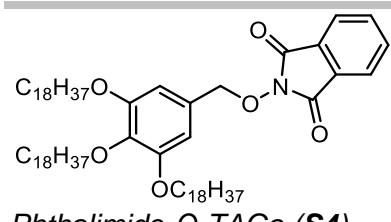

Phthalimide-O-TAGa (S4)

To a solution of $\mathbf{S} 3(20.0 \mathrm{~g}, 21.9 \mathrm{mmol}, 1.0 \mathrm{eq})$, triphenylphosphine $\left(\mathrm{PPh}_{3}\right)(11.5 \mathrm{~g}, 43.8 \mathrm{mmol}, 2.0 \mathrm{eq})$ and $\mathrm{N}$-hydroxyphthalimide $(7.14 \mathrm{~g}, 43.8 \mathrm{mmol}, 2.0 \mathrm{eq})$ in $\mathrm{CHCl}_{3}(274 \mathrm{~mL}, 0.08 \mathrm{M})$ was added dropwise diethyl azodicarboxylate (DEAD) (40\% toluene solution, $19.1 \mathrm{~g}, 43.8 \mathrm{mmol}, 2.0 \mathrm{eq})$ at $0{ }^{\circ} \mathrm{C}$ for $20 \mathrm{~min}$. The mixture was heated to room temperature and stirred for $5 \mathrm{~h}$. Methanol (MeOH) (1370 $\mathrm{mL}, 5$-fold excess of $\mathrm{CHCl}_{3}$ ) was added to the reaction mixture and the resulting heterogeneodus solution was stirred for a further $30 \mathrm{~min}$ at room temperature. The precipitate was filtered and washed with additional $\mathrm{MeOH}$ to afford phthalimide-O-TAGa (S4) (23.2 g, quant.) as a white powder. $\mathrm{mp} 81{ }^{\circ} \mathrm{C} ;{ }^{1} \mathrm{H}$ NMR $\left(500 \mathrm{MHz}, \mathrm{CDCl}_{3}\right) \delta 7.82-7.78(2 \mathrm{H}, \mathrm{m}), 7.74-7.71(2 \mathrm{H}, \mathrm{m}), 6.72(2 \mathrm{H}, \mathrm{s})$, $5.13(2 \mathrm{H}, \mathrm{s}), 3.97-3.92(6 \mathrm{H}, \mathrm{m}$, overlapped), 1.80-1.68 (6H, m, overlapped), 1.48-1.42 (6H, br m, overlapped), 1.35-1.22 (84H, br m, overlapped), $0.88(9 \mathrm{H}, \mathrm{t}, J=7.0 \mathrm{~Hz}) ;{ }^{13} \mathrm{C} \mathrm{NMR}\left(125 \mathrm{MHz}, \mathrm{CDCl}_{3}\right.$, peaks were complex because of rotamers) $\delta 163.6,153.3,139.1$, 134.5, 129.0, 128.6, $123.6108 .4,80.3,73.5,69.3,32.1,30.4,29.9-29.5$ (many signals overlapped), 26.2, 22.8, 14.3; IR (KBr) v (cm1) 2916, 2849, 1739, 1466, 1440, 1112, 700; HRMS (FAB, NBA + Na matrix) Calcd. for $\mathrm{C}_{69} \mathrm{H}_{119} \mathrm{NO}_{6} \mathrm{Na}: 1080.8935$ ([M+Na] $\left.{ }^{+}\right)$, Found: 1080.8942.

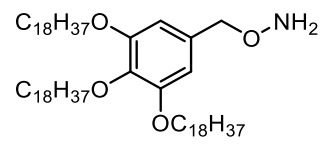

$\mathrm{NH}_{2}-\mathrm{O}-\mathrm{TAGa}$ (S5)

To a solution of $\mathbf{S} 4(23.2 \mathrm{~g}, 21.9 \mathrm{mmol}, 1.0 \mathrm{eq})$ in ethanol $(\mathrm{EtOH})(730 \mathrm{~mL}, 0.03 \mathrm{M})$ was added dropwise $\mathrm{N}_{2} \mathrm{H}_{4} \cdot \mathrm{H}_{2} \mathrm{O}(11.0 \mathrm{~g}, 219$ $\mathrm{mmol}, 10 \mathrm{eq}$ ) at room temperature. The mixture was heated to reflux and stirred for $8 \mathrm{~h}$. After cooling to room temperature, the resulting heterogeneous solution was stirred for a further $30 \mathrm{~min}$ at room temperature. The precipitate was filtered and washed with additional EtOH to afford $\mathrm{NH}_{2}-\mathrm{O}-\mathrm{TAGa}$ (S5) $\left(20.3 \mathrm{~g}\right.$, quant.) as a white powder. mp $69-70{ }^{\circ} \mathrm{C} ;{ }^{1} \mathrm{H} \mathrm{NMR}(500 \mathrm{MHz} \mathrm{CDCl} 3) \delta 6.55(2 \mathrm{H}$, s), $5.39(2 \mathrm{H}, \mathrm{br} \mathrm{s}), 4.59(2 \mathrm{H}, \mathrm{s}), 3.98-3.92(6 \mathrm{H}, \mathrm{m}$, overlapped), 1.82-1.71 $(6 \mathrm{H}, \mathrm{m}$, overlapped $), 1.50-1.43(6 \mathrm{H}$, br m, overlapped), 1.37-1.22 (84H, br m, overlapped), $0.88(9 \mathrm{H}, \mathrm{t}, J=7.0 \mathrm{~Hz}) ;{ }^{13} \mathrm{C} \mathrm{NMR}\left(125 \mathrm{MHz}, \mathrm{CDCl}_{3}\right) \delta 153.4,138.1,132.4,106.8,78.5,73.5$, 69.2, 32.1, 30.5, 29.9-29.5 (many signals overlapped), 26.29, 26.26, 22.8, 14.3; IR (KBr) $v\left(\mathrm{~cm}^{-1}\right) 2914,2848,1596,1469,1243$, 1128, 718; HRMS $\left(E S{ }^{+}\right)$Calcd. for $\mathrm{C}_{61} \mathrm{H}_{118} \mathrm{NO}_{4}: 928.9061\left([\mathrm{M}+\mathrm{H}]^{+}\right)$, Found: 928.9056.

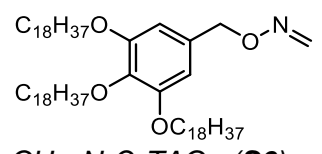

$\mathrm{CH}_{2}=\mathrm{N}-\mathrm{O}-\mathrm{TAGa}(\mathbf{S 6})$

A mixture of $\mathbf{S} 5(20.3 \mathrm{~g}, 21.9 \mathrm{mmol}, 1.0 \mathrm{eq})$ and paraformaldehyde $\left(\left(\mathrm{CH}_{2} \mathrm{O}\right)_{\mathrm{n}}\right)(6.56 \mathrm{~g}, 219 \mathrm{mmol}, 10 \mathrm{eq})$ in $\mathrm{CHCl}_{3} / \mathrm{MeOH}^{2} 5 / 1$ $(375 \mathrm{~mL}, 0.06 \mathrm{M})$ was heated to reflux and stirred for $3 \mathrm{~h}$. After cooling to room temperature, $\mathrm{MeOH}(1560 \mathrm{~mL}, 5$-fold excess of $\mathrm{CHCl}_{3}$ ) was added to the reaction mixture and was stirred for a further $30 \mathrm{~min}$ at room temperature. The precipitate was filtered and washed with additional $\mathrm{MeOH}$ to afford $\mathrm{CH}_{2}=\mathrm{N}-\mathrm{O}-\mathrm{TAGa}(\mathbf{S} 6)\left(20.6 \mathrm{~g}\right.$, quant.) as a white powder. $\mathrm{mp} 71-72{ }^{\circ} \mathrm{C} ;{ }^{1} \mathrm{H} \mathrm{NMR}(500 \mathrm{MHz}$, $\left.\mathrm{CDCl}_{3}\right) \delta 7.09(1 \mathrm{H}, \mathrm{d}, J=8.5 \mathrm{~Hz}), 6.55(2 \mathrm{H}, \mathrm{s}), 6.48(1 \mathrm{H}, \mathrm{d}, J=8.5 \mathrm{~Hz}), 5.02(2 \mathrm{H}, \mathrm{s}), 3.98-3.92(6 \mathrm{H}, \mathrm{m}$, overlapped $), 1.82-1.70(6 \mathrm{H}$, m, overlapped), 1.49-1.43 (6H, br m, overlapped), 1.35-1.22 (84H, br m, overlapped), $0.88(9 \mathrm{H}, \mathrm{t}, \mathrm{J}=7.5 \mathrm{~Hz}) ;{ }^{13} \mathrm{C} \mathrm{NMR}(125 \mathrm{MHz}$ $\left.\mathrm{CDCl}_{3}\right) \delta 153.3,138.1,137.8,132.4,106.9,76.6,73.5,69.2,32.1,30.5,29.9-29.2$ (many signals overlapped), 26.29, 26.26, 22.8, 14.3; IR $(\mathrm{KBr}) \vee\left(\mathrm{cm}^{-1}\right)$ 2914, 2847, 1596, 1469, 1243, 1129, 717; HRMS data is not available because the parent peak was not detected by ESI nor FAB. Only hydrolyzed peaks $\left(928.9\left([\mathbf{S} 5+\mathrm{H}]^{+}\right)\right.$and $\left.959.9\left([\mathbf{S} 5+\mathrm{Na}]^{+}\right)\right)$were observed.

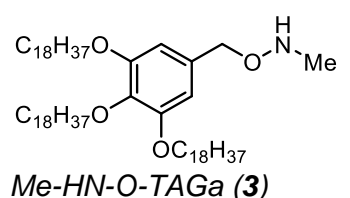

Me-HN-O-TAGa (3)

To a solution of $\mathbf{S 6}(17.6 \mathrm{~g}, 18.7 \mathrm{mmol}, 1.0 \mathrm{eq})$ and $\mathrm{NaBH}_{3} \mathrm{CN}(3.53 \mathrm{~g}, 56.1 \mathrm{mmol}, 3.0 \mathrm{eq})$ in EtOH/THF = $1 / 1(535 \mathrm{~mL}, 0.035 \mathrm{M})$ was added dropwise conc. $\mathrm{HCl}(4.29 \mathrm{~g})$ at room temperature until the $\mathrm{pH}$ reached around 3 . After stirring for $2 \mathrm{~h}, \mathrm{MeOH}(1338 \mathrm{~mL}, 5-$ fold excess of THF) was added to the mixture and the resulting heterogeneous solution was stirred for a further 30 min at room temperature. The precipitate was filtered and washed with additional MeOH to afford Me-HN-O-TAGa (3) (17.7 g, app. quant.) as a white powder. Additional purification was performed by column chromatography on silica gel $\left(\mathrm{CHCl}_{3} / n\right.$-hexane $=1 / 1$ to $5 / 1$ as eluent) to give highly pure $3(14.7 \mathrm{~g}, 83 \%)$ as a white powder. $\mathrm{mp} 53-54{ }^{\circ} \mathrm{C} ;{ }^{1} \mathrm{H}$ NMR $(500 \mathrm{MHz}, \mathrm{CDCl})_{3} \delta 6.55(2 \mathrm{H}, \mathrm{s}), 5.54(1 \mathrm{H}, \mathrm{br} \mathrm{s}), 4.61$ $(2 \mathrm{H}, \mathrm{s}), 3.98-3.91(6 \mathrm{H}, \mathrm{m}$, overlapped), $2.75(3 \mathrm{H}, \mathrm{s}), 1.82-1.70(6 \mathrm{H}, \mathrm{m}$, overlapped), 1.49-1.43 (6H, br m, overlapped), 1.37-1.22 (84H, br m, overlapped), $0.88(9 \mathrm{H}, \mathrm{t}, J=7.5 \mathrm{~Hz}) ;{ }^{13} \mathrm{C}$ NMR $\left(125 \mathrm{MHz}, \mathrm{CDCl}_{3}\right) \delta 153.3,137.9,133.1,106.8,76.1,73.5,69.2,39.4$, 32.1, 30.5, 29.9-29.5 (many signals overlapped), 26.29, 26.26, 22.8, 14.3; IR (KBr) $v\left(\mathrm{~cm}^{-1}\right)$ 2916, 2849, 1588, 1468, 1234, 1116, 720; HRMS (ESI+) Calcd. for $\mathrm{C}_{62} \mathrm{H}_{120} \mathrm{NO}_{4}: 942.9217\left([\mathrm{M}+\mathrm{H}]^{+}\right)$, Found: 942.9241. 


\section{Preparation of TAGb-type anchor molecule}

Scheme S2. Synthesis of TAGb-type anchor molecule<smiles>CCOc1ccc(CO)c(OCC)c1</smiles>

S7

II

HO-TAGb<smiles>O=C1c2ccccc2C(=O)N1O</smiles>

(crystallization)<smiles>CCOc1ccc(CON2C(=O)c3ccccc3C2=O)c(OCC)c1</smiles>

58
$\mathrm{N}_{2} \mathrm{H}_{4}-\mathrm{H}_{2} \mathrm{O}$

EtOH, reflux

quant.

(crystallization)<smiles>CCOc1ccc(CON)c(OCC)c1</smiles>

S9

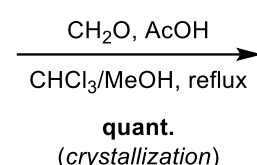

(crystallization)

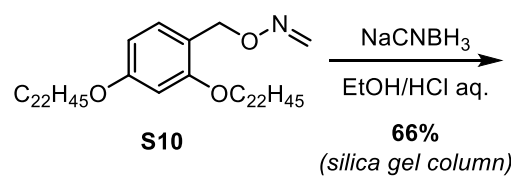

(silica gel column)<smiles>CCOc1ccc(CONC)c(OCC)c1</smiles><smiles>CCOc1ccc(CO)c(OCC)c1</smiles>

2,4-Bis(docosyloxy)benzyl alcohol (S7) (HO-TAGb) ${ }^{[5]}$

HO-TAGb (S7) (11.4 g, $15.1 \mathrm{mmol}$ ) was prepared by the same procedure as HO-TAGa (S3) described above as a white powder. mp 69-70 ${ }^{\circ} \mathrm{C} ;{ }^{1} \mathrm{H}$ NMR $\left(500 \mathrm{MHz}, \mathrm{CDCl}_{3}\right) \delta 7.13(1 \mathrm{H}, \mathrm{d}, J=8.5 \mathrm{~Hz}), 6.45(1 \mathrm{H}, \mathrm{d}, J=2.5 \mathrm{~Hz}), 6.42(1 \mathrm{H}, \mathrm{dd}, J=8.0,2.0 \mathrm{~Hz}), 4.61(2 \mathrm{H}$, d, $J=6.5 \mathrm{~Hz}), 3.98(2 \mathrm{H}, \mathrm{t}, J=6.5 \mathrm{~Hz}), 3.93(2 \mathrm{H}, \mathrm{t}, J=6.5 \mathrm{~Hz}), 2.27(1 \mathrm{H}, \mathrm{t}, J=6.5 \mathrm{~Hz}), 1.83-1.74(4 \mathrm{H}, \mathrm{m}$, overlapped $), 1.48-1.41(4 \mathrm{H}$, br m, overlapped), $1.37-1.22\left(72 \mathrm{H}\right.$, br m, overlapped), $0.88(6 \mathrm{H}, \mathrm{t}, J=7.0 \mathrm{~Hz}) ;{ }^{13} \mathrm{C} \mathrm{NMR}\left(125 \mathrm{MHz}, \mathrm{CDCl}_{3}\right.$, peaks were complex because of rotamers) $\delta 160.3,158.2,129.7,121.8,104.6,99.9,68.3,68.1,62.2,32.1,29.9-29.4$ (many signals overlapped), 26.3, 26.2, 22.8, 14.3; IR $(\mathrm{KBr}) \vee\left(\mathrm{cm}^{-1}\right)$ 2916, 2848, 1614, 1470, 1180, 1121, 718; HRMS (FAB, NBA + Na matrix) Calcd. for $\mathrm{C}_{51} \mathrm{H}_{96} \mathrm{O}_{3} \mathrm{Na}$ : $779.7257\left([\mathrm{M}+\mathrm{Na}]^{+}\right)$, Found: 779.7258 .<smiles>CCOc1ccc(CON2C(=O)c3ccccc3C2=O)c(OCC)c1</smiles>

Phthalimide-O-TAGb (S8)

Following the same procedure described for phthalimide-O-TAGa (S4), HO-TAGb (S7) $(6.30 \mathrm{~g}, 8.3 \mathrm{mmol})$ was converted to phthalimide-O-TAGa (S4) $(7.40 \mathrm{~g}, 99 \%)$ as a white powder. $\mathrm{mp} 85-86{ }^{\circ} \mathrm{C} ;{ }^{1} \mathrm{H}$ NMR $\left(500 \mathrm{MHz}, \mathrm{CDCl}_{3}\right) \delta 7.80-7.76(2 \mathrm{H}, \mathrm{m}), 7.72-7.68$ $(2 \mathrm{H}, \mathrm{m}), 7.32(1 \mathrm{H}, \mathrm{d}, J=8.5 \mathrm{~Hz}), 6.43(1 \mathrm{H}, \mathrm{dd}, J=8.5,2.5 \mathrm{~Hz}), 6.37(1 \mathrm{H}, \mathrm{d}, J=2.0 \mathrm{~Hz}), 5.20(2 \mathrm{H}, \mathrm{s}), 3.92(2 \mathrm{H}, \mathrm{t}, J=6.5 \mathrm{~Hz}), 3.82$ $(2 \mathrm{H}, \mathrm{t}, J=6.5 \mathrm{~Hz}), 1.75(2 \mathrm{H}, \mathrm{m}), 1.68(2 \mathrm{H}, \mathrm{m}), 1.46-1.22\left(76 \mathrm{H}, \mathrm{br} \mathrm{m}\right.$, overlapped), $0.88(6 \mathrm{H}, \mathrm{t}, J=7.0 \mathrm{~Hz}) ;{ }^{13} \mathrm{C} \mathrm{NMR}(125 \mathrm{MHz}$, $\left.\mathrm{CDCl}_{3}\right) \delta 163.6,161.7,159.4,134.2,133.5,129.2,123.4,115.1,105.0,99.7,74.5,68.5,68.1,32.1,29.9-29.4$ (many signals overlapped), 29.1, 26.2, 26.1, 22.8, 14.3; IR (KBr) $v\left(\mathrm{~cm}^{-1}\right)$ 2913, 2849, 1745, 1471, 1187, 969, 695; HRMS (FAB, NBA + Na matrix) Calcd. for $\mathrm{C}_{59} \mathrm{H}_{99} \mathrm{NO}_{5} \mathrm{Na}: 924.7421\left([\mathrm{M}+\mathrm{Na}]^{+}\right)$, Found: 924.7426.<smiles>CCOc1ccc(CON)c(OCC)c1</smiles>

\section{$\mathrm{NH}_{2}-\mathrm{O}-\mathrm{TAGb}$ (S9)}

Following the same procedure described for $\mathrm{NH}_{2}$-O-TAGa (S5), phthalimide-O-TAGb (S8) $(7.30 \mathrm{~g}, 8.1 \mathrm{mmol})$ was converted to $\mathrm{NH}_{2}$-O-TAGb (S9) $\left(6.25 \mathrm{~g}\right.$, quant.) as a white powder. $\mathrm{mp} 71-73{ }^{\circ} \mathrm{C} ;{ }^{1} \mathrm{H}$ NMR $\left(500 \mathrm{MHz}, \mathrm{CDCl}_{3}\right) \delta 7.22(1 \mathrm{H}, \mathrm{m}), 6.46-6.43(2 \mathrm{H}, \mathrm{m}$, overlapped), $5.33(2 \mathrm{H}, \mathrm{s}), 4.69(2 \mathrm{H}, \mathrm{s}), 3.96-3.93(4 \mathrm{H}, \mathrm{m}$, overlapped), 1.82-1.74 $(4 \mathrm{H}, \mathrm{m}$, overlapped), $1.48-1.41(4 \mathrm{H}, \mathrm{br} \mathrm{m}$, overlapped), 1.37-1.22 $\left(72 \mathrm{H}\right.$, br m, overlapped), $0.88(6 \mathrm{H}, \mathrm{t}, J=7.0 \mathrm{~Hz}) ;{ }^{13} \mathrm{C}$ NMR $\left(125 \mathrm{MHz}, \mathrm{CDCl}_{3}\right.$, peaks were complex because of rotamers) $\delta 160.6,158.7,131.4,117.9,104.7,100.0,73.1,68.4,68.2,32.1,29.9-29.3$ (many signals overlapped), 26.23, 26.21, 22.8, 14.3; IR (KBr) v $\left(\mathrm{cm}^{-1}\right) 2915,2848,1618,1471,1200,1178,1041,717$; HRMS (ESI+) Calcd. for $\mathrm{C}_{51} \mathrm{H}_{98} \mathrm{NO}_{3}: 772.7547\left([\mathrm{M}+\mathrm{H}]^{+}\right)$, Found: 772.7550 .<smiles>C=NOCc1ccc(OCC)cc1OCC</smiles>

\section{$\mathrm{CH}_{2}=\mathrm{N}-\mathrm{O}-\mathrm{TAGb}(\mathrm{S} 10)$}

Following the same procedure described for $\mathrm{CH}_{2}=\mathrm{N}-\mathrm{O}-\mathrm{TAGa}(\mathbf{S 6}), \mathrm{NH}_{2}-\mathrm{O}-\mathrm{TAGb}$ (S9) (3.94 g, $\left.5.1 \mathrm{mmol}\right)$ was converted to $\mathrm{CH}_{2}=\mathrm{N}-\mathrm{O}-\mathrm{TAGb}$ (S10) (4.00 g, quant.) as a white powder. mp $64-65^{\circ} \mathrm{C} ;{ }^{1} \mathrm{H} \mathrm{NMR}(500 \mathrm{MHz}, \mathrm{CDCl} 3) \delta 7.22(1 \mathrm{H}, \mathrm{m}), 7.05(1 \mathrm{H}, \mathrm{d}, J=$ $8.5 \mathrm{~Hz}), 6.45-6.42(3 \mathrm{H}, \mathrm{m}$, overlapped), $5.12(2 \mathrm{H}, \mathrm{s}), 3.96-3.92(4 \mathrm{H}, \mathrm{m}$, overlapped), 1.81-1.74 (4H, m, overlapped), 1.48-1.41 (4H, br $\mathrm{m}$, overlapped), 1.37-1.22 (72H, br m, overlapped), $0.88(6 \mathrm{H}, \mathrm{t}, J=7.0 \mathrm{~Hz}) ;{ }^{13} \mathrm{C} \mathrm{NMR}\left(125 \mathrm{MHz}, \mathrm{CDCl}_{3}\right.$, peaks were complex because of rotamers) $\delta 160.6,158.4,137.1,131.1,118.1,104.7,100.0,71.2,68.3,68.2,32.1,29.9-29.3$ (many signals overlapped), 26.2, 22.8, 14.3; IR (KBr) v $\left(\mathrm{cm}^{-1}\right) 2916,2848,1613,1465,1289,1183,1004,817,718$; HRMS (ESI+) Calcd. for $\mathrm{C}_{52} \mathrm{H}_{97} \mathrm{NO}_{3} \mathrm{Na}$ $806.7366\left([\mathrm{M}+\mathrm{Na}]^{+}\right)$, Found: 806.7352 . 
Me-HN-O-TAGb (S11)

Following the same procedure described for Me-HN-O-TAGa (3), $\mathrm{CH}_{2}=\mathrm{N}-\mathrm{O}-\mathrm{TAGb}$ (S10) (3.95 g, $5.0 \mathrm{mmol}$ ) was converted to MeHN-O-TAGb (S11) (2.61 g after purification by column chromatography on silica gel $\left(\mathrm{CHCl}_{3} / n\right.$-hexane $=1 / 1$ to $5 / 1$ as eluent), $\left.66 \%\right)$ as a white powder. mp 60-61 ${ }^{\circ} \mathrm{C}$; ${ }^{1} \mathrm{H}$ NMR $\left(500 \mathrm{MHz}, \mathrm{CDCl}_{3}\right) \delta 7.22(1 \mathrm{H}, \mathrm{m}), 6.44-6.42(2 \mathrm{H}$, m, overlapped), $5.49(1 \mathrm{H}$, br s), $4.70(2 \mathrm{H}$, s), 3.96-3.91 (4H, m, overlapped), $2.75(3 \mathrm{H}, \mathrm{s}), 1.81-1.73(4 \mathrm{H}, \mathrm{m}$, overlapped), 1.48-1.41 (4H, br m, overlapped), 1.37-1.22 (72H, br $\mathrm{m}$, overlapped), $0.88(6 \mathrm{H}, \mathrm{t}, J=7.5 \mathrm{~Hz}) ;{ }^{13} \mathrm{C} \mathrm{NMR}\left(125 \mathrm{MHz} \mathrm{CDCl}_{3}\right.$, peaks were complex because of rotamers) $\delta 160.4,158.5$, $131.2,118.5,104.7,100.0,70.3,68.3,68.2,39.3,32.1,29.9-29.4$ (many signals overlapped), 26.3, 26.2, 22.8, 14.3; IR (KBr) v (cm ${ }^{-1}$ ) 2915, 2848, 1613, 1466, 1287, 1178, 718; HRMS (ESI $\left.{ }^{+}\right)$Calcd. for $\mathrm{C}_{52} \mathrm{H}_{100} \mathrm{NO}_{3}: 786.7703\left([\mathrm{M}+\mathrm{H}]^{+}\right)$, Found: 786.7685.

\section{Peptide elongations}

\section{General procedure for elongation of peptide chain (condensation with Fmoc-protected amino acid)}

To a solution of free amine (1.0 eq) in DCM $(0.05 \mathrm{M}$ for substrate) was added Fmoc protected amino acid (1.1 eq), 1hydroxybenzotriazole (HOBt) (1.2 eq), and $N, N^{\prime}$-diisopropylcarbodiimide (DIC) (1.2 eq (2.3 eq when Fmoc protected amino acid was monohydrate)) at room temperature to $40{ }^{\circ} \mathrm{C}$ and stirred until the reaction completed (1.5 to $5 \mathrm{~h}$ ). The reaction mixture was subsequently cooled to $0^{\circ} \mathrm{C}$ and $\mathrm{MeOH}$ (generally 5 -fold excess of $\mathrm{DCM}$ ) was added. The resulting heterogeneous solution was stirred for a further $30 \mathrm{~min}$ at $0{ }^{\circ} \mathrm{C}$, and the precipitate was filtered and washed with additional $\mathrm{MeOH}$ to afford the corresponding Fmoc protected peptide with an anchor molecule as a white to off-white powder.

\section{General procedure for Fmoc deprotection}

The Fmoc protected amino acid or peptide was dissolved into or $10 \%$ piperidine/DCM or $1 \%$ piperidine/1\% 1,8-diazabicyclo[5.4.0]7-undecene (DBU) $/ \mathrm{CHCl}_{3}(0.036 \mathrm{M}$ for substrate) at room temperature, and the solution was stirred until the reaction was completed (generally $0.5 \mathrm{~h}$ to $2 \mathrm{~h}$ ). The reaction mixture was subsequently cooled to $0{ }^{\circ} \mathrm{C}$ and $\mathrm{MeOH}$ (generally 5 -fold excess of $\mathrm{DCM}^{\circ}$ or $\mathrm{CHCl}_{3}$ ) was added. The resulting heterogeneous solution was stirred for a further $30 \mathrm{~min}$ at $0{ }^{\circ} \mathrm{C}$, and the precipitate was filtered and washed with additional $\mathrm{MeOH}$ to afford the corresponding amine as a white to off-white powder.

\section{Using TAGa-type anchor molecule}

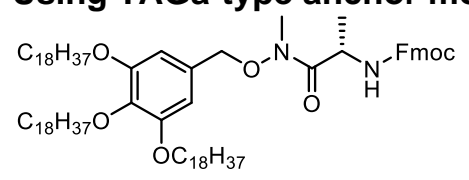

Fmoc-Ala-(Me)N-O-TAGa (S12)

Following the general procedure described for condensation, Me-HN-O-TAGa (3) $(5.00 \mathrm{~g}, 5.3 \mathrm{mmol})$ was converted to Fmoc-Ala(Me)N-O-TAGa (S12) (6.56 g, quant.) as a white powder. [a $]_{D}^{24.3}=+12.1\left(c 0.1, \mathrm{CHCl}_{3}\right) ; \mathrm{mp} 56-59{ }^{\circ} \mathrm{C} ;{ }^{1} \mathrm{H} \mathrm{NMR}(500 \mathrm{MHz}, \mathrm{CDCl})_{3} \delta$ $7.77(2 \mathrm{H}, \mathrm{d}, J=7.5 \mathrm{~Hz}), 7.62(2 \mathrm{H}, \mathrm{t}, J=7.5 \mathrm{~Hz}), 7.40(2 \mathrm{H}, \mathrm{t}, J=7.5 \mathrm{~Hz}), 7.32(2 \mathrm{H}, \mathrm{t}, J=7.5 \mathrm{~Hz}), 6.63(2 \mathrm{H}, \mathrm{s}), 5.62(1 \mathrm{H}, \mathrm{br} d, J=8.5$ $\mathrm{Hz}), 4.88(1 \mathrm{H}, \mathrm{br} \mathrm{m}), 4.86(2 \mathrm{H}, \mathrm{s}), 4.40-4.34(2 \mathrm{H}, \mathrm{m}$, overlapped), $4.24(1 \mathrm{H}, \mathrm{t}, J=7.5 \mathrm{~Hz}), 4.00-3.94(6 \mathrm{H}$, m, overlapped), $3.24(3 \mathrm{H}$, s ), $1.83-1.71\left(6 \mathrm{H}, \mathrm{m}\right.$, overlapped), $1.50-1.44\left(6 \mathrm{H}\right.$, br m, overlapped), 1.37-1.22 (87H, br m, overlapped), $0.89(9 \mathrm{H}, \mathrm{t}, \mathrm{J}=7.0 \mathrm{~Hz}) ;{ }^{13} \mathrm{C}$ $\operatorname{NMR}\left(125 \mathrm{MHz}, \mathrm{CDCl}_{3}\right) \delta 173.9,155.9,153.5,144.1,143.9,141.40,141.38,138.9,129.1,127.8,127.2,125.29,125.25,120.1$, 107.8, 77.7, 73.5, 69.3, 67.1, 47.4, 47.3, 33.9, 32.1, 30.5, 29.9-29.5 (many signals overlapped), 26.2, 22.8, 18.6, 14.2; IR (KBr) $v\left(\mathrm{~cm}^{-}\right.$ $\left.{ }^{1}\right)$ 2916, 2849, 1658, 1467, 1243, 1119, 740, 720; HRMS (FAB, NBA + Nal matrix) Calcd. for $\mathrm{C}_{80} \mathrm{H}_{134} \mathrm{O}_{7} \mathrm{~N}_{2} \mathrm{Na}: 1258.0089\left([\mathrm{M}+\mathrm{Na}]^{+}\right)$, Found: 1258.0090 .

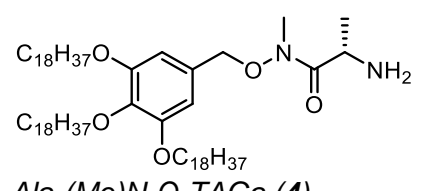

Ala-(Me)N-O-TAGa (4)

Following the general procedure described for Fmoc deprotection, Fmoc-Ala-(Me)N-O-TAGa (S12) (3.90 g, $3.2 \mathrm{mmol})$ was converted to Ala-(Me)N-O-TAGa (4) $\left(3.20 \mathrm{~g}\right.$, quant.) as a white powder. $[\alpha]_{D}{ }^{24.3}=+5.6\left(c 0.1, \mathrm{CHCl}_{3}\right) ; \mathrm{mp} 64-65^{\circ} \mathrm{C} ;{ }^{1} \mathrm{H} \mathrm{NMR}(500$ $\left.\mathrm{MHz}, \mathrm{CDCl}_{3}\right) \delta 6.53(2 \mathrm{H}, \mathrm{s}), 4.74(2 \mathrm{H}$, app dd, $J=18.5,10.5 \mathrm{~Hz}), 3.97-3.93(6 \mathrm{H}, \mathrm{m}$, overlapped), $3.85(1 \mathrm{H}, \mathrm{br} \mathrm{d}, J=6.0 \mathrm{~Hz}), 3.22$ $(3 \mathrm{H}, \mathrm{s}), 1.82-1.70(6 \mathrm{H}, \mathrm{m}$, overlapped), $1.49-1.43(6 \mathrm{H}, \mathrm{br} \mathrm{m}$, overlapped), $1.37-1.22(87 \mathrm{H}, \mathrm{br} \mathrm{m}$, overlapped), $0.87(9 \mathrm{H}, \mathrm{t}, J=7.0 \mathrm{~Hz})$; ${ }^{13} \mathrm{C}$ NMR $\left(125 \mathrm{MHz}, \mathrm{CDCl}_{3}\right) \delta 178.2,153.5,139.0,129.3,107.7,77.0,73.6,69.4,47.3,34.0,32.1,30.5,29.9-29.5$ (many signals overlapped), 26.2, 22.8, 20.8, 14.3; IR (KBr) $v\left(\mathrm{~cm}^{-1}\right) 2915,2848,1641,1469,1335,1240,1123,719 ; \mathrm{HRMS}$ (FAB, NBA + Nal matrix) Calcd. for $\mathrm{C}_{65} \mathrm{H}_{124} \mathrm{O}_{5} \mathrm{~N}_{2} \mathrm{Na}$ : $1035.9408\left([\mathrm{M}+\mathrm{Na}]^{+}\right)$, Found: 1035.9396.

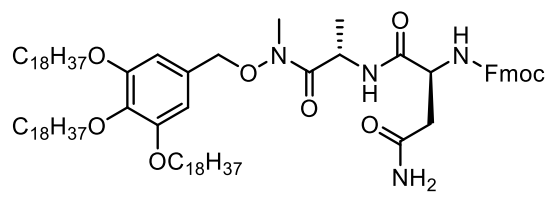

Fmoc-Asn-Ala-(Me)N-O-TAGa (S13)

Following the general procedure described for condensation, Ala-(Me)N-O-TAGa (4) $(5.00 \mathrm{~g}, 4.9 \mathrm{mmol})$ was converted to FmocAsn-Ala-(Me)N-O-TAGa (S13) $(6.54 \mathrm{~g}, 98 \%)$ as a white powder. [a $]_{\mathrm{D}}^{24.4}=+11.9\left(\mathrm{c} 0.1, \mathrm{CHCl}_{3}\right) ; \mathrm{mp} 134-135^{\circ} \mathrm{C} ;{ }^{1} \mathrm{H} \mathrm{NMR}(500 \mathrm{MHz}$, $\left.\mathrm{CDCl}_{3}\right) \delta 7.75(2 \mathrm{H}, \mathrm{d}, J=7.5 \mathrm{~Hz}), 7.60(2 \mathrm{H}, \mathrm{br} \mathrm{dd}, J=7.0,4.5 \mathrm{~Hz}), 7.54(1 \mathrm{H}, \mathrm{br} \mathrm{d}, J=7.5 \mathrm{~Hz}), 7.39(2 \mathrm{H}, \mathrm{t}, J=7.5 \mathrm{~Hz}), 7.31(2 \mathrm{H}, \mathrm{td}, J$ $=7.5,1.0 \mathrm{~Hz}), 6.58(2 \mathrm{H}, \mathrm{s}), 6.36(1 \mathrm{H}, \mathrm{br} \mathrm{d}, J=7.5 \mathrm{~Hz}), 6.09(1 \mathrm{H}, \mathrm{br} \mathrm{s}), 5.63(1 \mathrm{H}, \mathrm{br} \mathrm{s}), 4.93(1 \mathrm{H}, \mathrm{br} \mathrm{m}), 4.84(2 \mathrm{H}, \mathrm{app} \mathrm{dd}, J=19.5$, 
$10.0 \mathrm{~Hz}), 4.60(1 \mathrm{H}, \mathrm{br} \mathrm{m}), 4.41-4.35(2 \mathrm{H}, \mathrm{m}$, overlapped), $4.22(1 \mathrm{H}, \mathrm{t}, J=7.5 \mathrm{~Hz}), 3.98-3.93(6 \mathrm{H}, \mathrm{m}$, overlapped), $3.20(3 \mathrm{H}, \mathrm{s}), 2.91$ $(1 \mathrm{H}$, br m), $2.63(1 \mathrm{H} \mathrm{br} \mathrm{m}), 1.82-1.71(6 \mathrm{H}, \mathrm{m}$, overlapped $), 1.49-1.44(6 \mathrm{H}, \mathrm{br} \mathrm{m}$, overlapped $), 1.34-1.22(87 \mathrm{H}$, br m, overlapped), 0.88 $(9 \mathrm{H}, \mathrm{t}, J=7.0 \mathrm{~Hz}) ;{ }^{13} \mathrm{C}$ NMR $\left(125 \mathrm{MHz}, \mathrm{CDCl}_{3}\right) \delta 173.4$ (two signals), 170.5, 156.2, 153.5, 144.0, 143.8, 141.4, 138.7, 129.3, 127.8, 127.2, 125.3, 120.1, 107.7, 73.6, 69.3, 67.4, 51.3, 47.2, 46.5, 37.7, 34.0, 32.1, 30.5, 29.9-29.5 (many signals overlapped), 26.3, 22.8, 17.7, 14.3; IR (KBr) v $\left(\mathrm{cm}^{-1}\right) 3403,3298,2916,2848,1668,1655,1468,1262,1125,990,740,719$; HRMS (FAB, NBA + Nal matrix) Calcd. for $\mathrm{C}_{84} \mathrm{H}_{140} \mathrm{O}_{9} \mathrm{~N}_{4} \mathrm{Na}: 1372.0518\left([\mathrm{M}+\mathrm{Na}]^{+}\right)$, Found: 1372.0538.

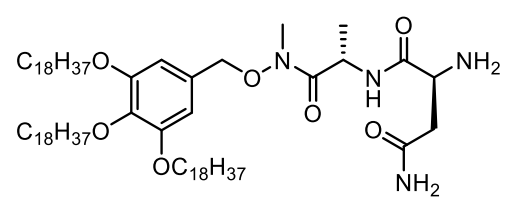

Asn-Ala-(Me)N-O-TAGa (5)

Following the general procedure described for Fmoc deprotection, Fmoc-Asn-Ala-(Me)N-O-TAGa (S13) (5.00 g, $3.7 \mathrm{mmol})$ was converted to Asn-Ala-(Me)N-O-TAGa (5) (4.18 g, quant.) as a white powder. [a] $]_{\mathrm{D}}^{24.5}=-4.2\left(\mathrm{c} 0.1, \mathrm{CHCl}_{3}\right) ; \mathrm{mp}^{67-69}{ }^{\circ} \mathrm{C} ;{ }^{1} \mathrm{H} \mathrm{NMR}$ $\left(500 \mathrm{MHz}, \mathrm{CDCl}_{3}\right) \delta 7.92(1 \mathrm{H}$, br d, $J=7.5 \mathrm{~Hz}), 6.59(2 \mathrm{H}, \mathrm{s}), 6.35(1 \mathrm{H}$, br s$), 5.62(1 \mathrm{H}$, br s), $4.97(1 \mathrm{H}$, br m$), 4.84(2 \mathrm{H}$, app dd, $J=$ 21.5, $10.5 \mathrm{~Hz}), 3.98-3.92(6 \mathrm{H}, \mathrm{m}$, overlapped), $3.70(1 \mathrm{H}, \mathrm{m}), 3.20(3 \mathrm{H}, \mathrm{s}), 2.67(1 \mathrm{H}, \mathrm{br} \mathrm{m}), 2.53(1 \mathrm{H}, \mathrm{br} \mathrm{m}), 1.82-1.70(6 \mathrm{H}, \mathrm{m}$, overlapped), 1.49-1.43 (6H, br m, overlapped), 1.37-1.21 (87H, br m, overlapped), $0.87(9 \mathrm{H}, \mathrm{t}, J=7.0 \mathrm{~Hz}) ;{ }^{13} \mathrm{C} \mathrm{NMR}(125 \mathrm{MHz}$ $\mathrm{CDCl}_{3}$, Signals were complex due to the rotamers.) $\delta 173.75,173.69,173.55,173.50,173.45,153.4,138.8,129.3,107.8,77.4,73.6$, 69.3, 52.4, 45.8, 45.6, 40.6, 34.0, 32.1, 30.5, 29.9-29.5 (many signals overlapped), 26.3, 22.8, 18.08, 18.05, 14.3; IR (KBr) $v\left(\mathrm{~cm}^{-1}\right)$ 3366, 2916, 2848, 1659, 1468, 1334, 1122, 719; HRMS (FAB, NBA matrix) Calcd. for $\mathrm{C}_{69} \mathrm{H}_{131} \mathrm{O}_{7} \mathrm{~N}_{4}: 1128.0018\left([\mathrm{M}+\mathrm{H}]^{+}\right)$, Found: 1128.0023.

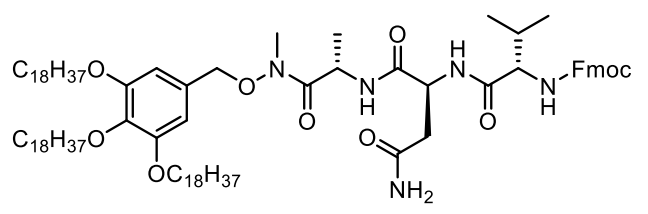

Fmoc-Val-Asn-Ala-(Me)N-O-TAGa (S14)

Following the general procedure described for condensation, Asn-Ala-(Me)N-O-TAGa (5) $(4.10 \mathrm{~g}, 3.6 \mathrm{mmol})$ was converted to Fmoc-Val-Asn-Ala-(Me)N-O-TAGa (S14) $(5.27 \mathrm{~g} \text {, quant.) as a white powder. [a] }]^{24.5}=-0.1\left(\mathrm{c} 0.1, \mathrm{CHCl}_{3}\right) ; \mathrm{mp}^{174}-179{ }^{\circ} \mathrm{C} ;{ }^{1} \mathrm{H} \mathrm{NMR}$ $\left(500 \mathrm{MHz}, \mathrm{CDCl}_{3}\right.$, Signals were complex due to rotamers of the peptide bonds.) $\delta 7.75(1 \mathrm{H}, \mathrm{br} \mathrm{s}), 7.73(2 \mathrm{H}, \mathrm{dd}, \mathrm{J}=7.5,4.0 \mathrm{~Hz}), 7.63$ $(1 \mathrm{H}$, br d, $J=7.0 \mathrm{~Hz}), 7.58(2 \mathrm{H}$, br d, $J=7.5 \mathrm{~Hz}), 7.36(2 \mathrm{H}$, app q, $J=7.0 \mathrm{~Hz}), 7.28(2 \mathrm{H}, \mathrm{br} \mathrm{t}, J=7.5 \mathrm{~Hz}), 6.55(2 \mathrm{H}, \mathrm{s}), 6.30(1 \mathrm{H}, \mathrm{br}$ s), $5.85(1 \mathrm{H}, \mathrm{br} \mathrm{s}), 5.75(1 \mathrm{H}, \mathrm{br} \mathrm{d}, J=8.0 \mathrm{~Hz}), 4.93-4.77(4 \mathrm{H}, \mathrm{br} \mathrm{m}$, overlapped), $4.40(1 \mathrm{H}, \mathrm{br} \mathrm{dd}, J=10.5,8.0 \mathrm{~Hz}), 4.31(1 \mathrm{H}, \mathrm{br} \mathrm{dd}, J$ $=10.5,7.5 \mathrm{~Hz}), 4.19(1 \mathrm{H}, \mathrm{br} \mathrm{t}, J=7.0 \mathrm{~Hz}), 4.14(1 \mathrm{H}, \mathrm{br} \mathrm{t}, J=7.0 \mathrm{~Hz}), 3.97-3.92(6 \mathrm{H}, \mathrm{br} \mathrm{m}$, overlapped), $3.17(3 \mathrm{H}, \mathrm{s}), 2.83(1 \mathrm{H}, \mathrm{br} \mathrm{m})$, $2.62(1 \mathrm{H}$, br m), $2.16(1 \mathrm{H}, \mathrm{br} \mathrm{m}), 1.81-1.71(6 \mathrm{H}, \mathrm{m}$, overlapped), 1.49-1.43 $(6 \mathrm{H}$, br m, overlapped), 1.35-1.22 (87H, br m, overlapped), $0.99(3 \mathrm{H}, \mathrm{d}, J=6.0 \mathrm{~Hz}), 0.94(3 \mathrm{H}, \mathrm{d}, J=7.0 \mathrm{~Hz}), 0.88(9 \mathrm{H}, \mathrm{t}, J=7.0 \mathrm{~Hz}) ;{ }^{13} \mathrm{C} \mathrm{NMR}\left(125 \mathrm{MHz}, \mathrm{CDCl}_{3}\right.$, Signals were complex due to the rotamers.) $\delta 173.6,173.4,171.6,170.2,156.7,153.4,144.0,143.9,141.4,138.8,129.3,127.8,127.22,127.18,125.30,125.25$, $120.07,120.05,107.7,73.6,69.3,67.3,60.4,49.8,47.3,46.4,37.1,34.0,32.1,31.5,30.5,29.9-29.5$ (many signals overlapped), 26.3, 22.8, 19.4, 17.9, 17.6, 14.3; IR (KBr) $v\left(\mathrm{~cm}^{-1}\right)$ 3272, 2917, 2849, 1639, 1468, 1247,1120, 719; HRMS (FAB, NBA + Nal matrix) Calcd. for $\mathrm{C}_{89} \mathrm{H}_{149} \mathrm{O}_{10} \mathrm{~N}_{5} \mathrm{Na}: 1471.1202\left([\mathrm{M}+\mathrm{Na}]^{+}\right)$, Found: 1471.0200 .

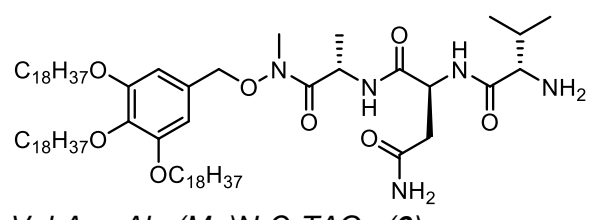

Val-Asn-Ala-(Me)N-O-TAGa (6)

Following the general procedure described for Fmoc deprotection, Fmoc-Val-Asn-Ala-(Me)N-O-TAGa (S14) (5.21 g, $3.6 \mathrm{mmol})$ was converted to Val-Asn-Ala-(Me)N-O-TAGa (6) (4.41 g, quant.) as a white powder. [a $]_{\mathrm{D}}^{24.6}=-2.8\left(\mathrm{c} 0.1, \mathrm{CHCl}_{3}\right) ; \mathrm{mp}^{81-83}{ }^{\circ} \mathrm{C} ;{ }^{1} \mathrm{H}$ NMR $\left(500 \mathrm{MHz}, \mathrm{CDCl}_{3}\right.$, Signals were complex due to rotamers of the peptide bonds.) $\delta 8.40(1 \mathrm{H}, \mathrm{d}, \mathrm{J}=8.0 \mathrm{~Hz}), 7.66(1 \mathrm{H}, \mathrm{d}, \mathrm{J}=7.0$ $\mathrm{Hz}), 6.57(2 \mathrm{H}, \mathrm{s}), 6.47(1 \mathrm{H}$, br s$), 5.75(1 \mathrm{H}$, br s$), 4.92-4.77(4 \mathrm{H}, \mathrm{br} \mathrm{m}$, overlapped), 3.99-3.92 $(6 \mathrm{H}$, br m, overlapped $), 3.26(1 \mathrm{H}$, br d, $J=3.0 \mathrm{~Hz}), 3.18(3 \mathrm{H}, \mathrm{s}), 2.83(1 \mathrm{H}, \mathrm{m}), 2.63(1 \mathrm{H}, \mathrm{m}), 2.24(1 \mathrm{H}, \mathrm{m}), 1.82-1.70(6 \mathrm{H}, \mathrm{m}$, overlapped), 1.49-1.43 $(6 \mathrm{H}$, br m, overlapped), 1.37-1.22 (87H, br m, overlapped), $0.98(3 \mathrm{H}, \mathrm{d}, J=7.0 \mathrm{~Hz}), 0.87(9 \mathrm{H}, \mathrm{t}, J=7.5 \mathrm{~Hz}), 0.83(3 \mathrm{H}, \mathrm{d}, J=6.5 \mathrm{~Hz}) ;{ }^{13} \mathrm{C} \mathrm{NMR}(125 \mathrm{MHz}$, $\mathrm{CDCl}_{3}$, Signals were complex due to the rotamers.) $\delta 175.11,175.05,173.5,173.41,173.36,170.6,170.5,153.4,138.7,129.3$, 107.7, 73.6, 69.3, 60.3, 49.5, 49.4, 46.45, 46.35, 37.8, 34.0, 32.1, 31.2, 30.5, 29.9-29.5 (many signals overlapped), 26.3, 22.8, 19.8, 17.6, 16.3, 14.2; IR $(\mathrm{KBr}) \vee\left(\mathrm{cm}^{-1}\right) 3277,2916,2849,1637,1468,1236,1121,720$; HRMS (FAB, NBA matrix) Calcd. for $\mathrm{C}_{74} \mathrm{H}_{140} \mathrm{O}_{8} \mathrm{~N}_{5}$ : $1227.0702\left([\mathrm{M}+\mathrm{H}]^{+}\right)$, Found: 1227.0693 .

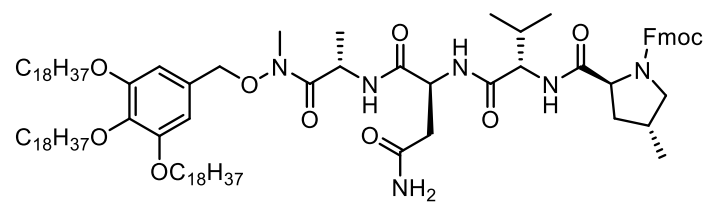

Fmoc-4-MePro-Val-Asn-Ala-(Me)N-O-TAGa (S15)

Following the general procedure described for condensation, Val-Asn-Ala-(Me)N-O-TAGa (6) (4.40 g, $3.6 \mathrm{mmol})$ was converted to

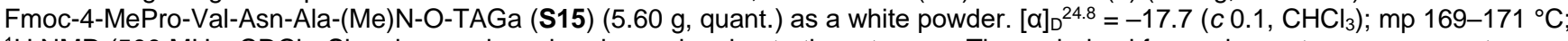
${ }^{1} \mathrm{H}$ NMR $\left(500 \mathrm{MHz}, \mathrm{CDCl}_{3}\right.$, Signals were broad and complex due to the rotamers. Those derived from minor rotamers were not described here.) $\delta 7.76(2 \mathrm{H}, \mathrm{br} \mathrm{d}, J=7.5 \mathrm{~Hz}), 7.70(1 \mathrm{H}, \mathrm{br} \mathrm{d}, J=7.5 \mathrm{~Hz}), 7.58(2 \mathrm{H}, \mathrm{br} \mathrm{d}, J=7.0 \mathrm{~Hz}), 7.56(1 \mathrm{H}, \mathrm{br} \mathrm{m}), 7.39(2 \mathrm{H}, \mathrm{br} \mathrm{t}, J$ 
$=7.5 \mathrm{~Hz}), 7.31(2 \mathrm{H}, \mathrm{td}, J=7.5,1.5 \mathrm{~Hz}), 6.57(2 \mathrm{H}, \mathrm{br} \mathrm{s}), 6.45(1 \mathrm{H}, \mathrm{br} \mathrm{s}), 5.93(1 \mathrm{H}, \mathrm{br} \mathrm{s}), 5.51(1 \mathrm{H}$, ap p br d, J= $48 \mathrm{~Hz}), 4.92(1 \mathrm{H}, \mathrm{br}$ s), 4.86-4.78 (3H, m, overlapped), 4.46-4.33 (3H, br m, overlapped), $4.22(1 \mathrm{H}$, br t, $J=7.0 \mathrm{~Hz}), 3.97-3.92(6 \mathrm{H}, \mathrm{m}$, overlapped), 3.65 $(1 \mathrm{H}, \mathrm{brt}, J=8.5 \mathrm{~Hz}), 3.17(3 \mathrm{H}, \mathrm{s}), 2.95(1 \mathrm{H}, \mathrm{br} \mathrm{t}, J=10.0 \mathrm{~Hz}), 2.77(1 \mathrm{H}, \mathrm{br} \mathrm{m}), 2.66(1 \mathrm{H}, \mathrm{br} \mathrm{m}), 2.50-2.06(4 \mathrm{H}$, br m, overlapped), 1.81-1.70 $(6 \mathrm{H}, \mathrm{m}$, overlapped), $1.65(1 \mathrm{H}, \mathrm{br} \mathrm{m}), 1.49-1.43(6 \mathrm{H}, \mathrm{br} \mathrm{m}$, overlapped $), 1.37-1.22(87 \mathrm{H}$, br m, overlapped $), 1.06(3 \mathrm{H}, \mathrm{br} \mathrm{d}$, $J=6.5 \mathrm{~Hz}), 0.93(3 \mathrm{H}, \mathrm{d}, J=7.0 \mathrm{~Hz}), 0.87(12 \mathrm{H}$, app t, $J=7.0 \mathrm{~Hz}) ;{ }^{13} \mathrm{C} \mathrm{NMR}\left(125 \mathrm{MHz}, \mathrm{CDCl}_{3}\right.$, Signals were broad and complex due to the rotamers. Those derived from minor rotamers were not described here.) $\delta 173.4$ (two signals), 172.4, 171.3, 170.2, 156.3, 153.4, 144.0, 143.8, 141.4, 138.7, 129.4, 127.9, 127.2, 125.1, 120.1, 107.7, 73.6, 69.3, 68.1, 61.3, 58.7, 54.0, 49.9, 47.2, 46.3, 37.2, $36.7,34.1,32.7,32.0,30.9,30.5,29.9-29.5$ (many signals overlapped), 26.3, 22.8, 19.5, 17.8, 17.7, 17.3, 14.2; IR $(\mathrm{KBr}) v\left(\mathrm{~cm}{ }^{-1}\right)$ 3272, 2916, 2849, 1661, 1638, 1468, 1236, 1122, 739, 720; HRMS (FAB, NBA + Nal matrix) Calcd. for $\mathrm{C}_{95} \mathrm{H}_{158} \mathrm{O}_{11} \mathrm{~N}_{6} \mathrm{Na}_{1} 1582.1886$ $\left([\mathrm{M}+\mathrm{Na}]^{+}\right)$, Found: 1582.1896 .

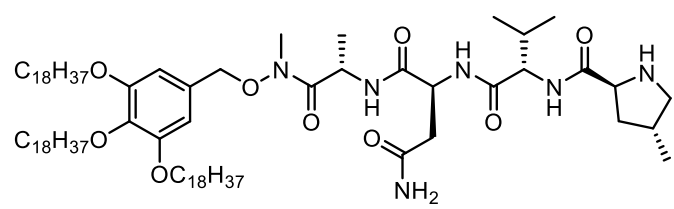

4-MePro-Val-Asn-Ala-(Me)N-O-TAGa (7)

Following the general procedure described for Fmoc deprotection, Fmoc-4-MePro-Val-Asn-Ala-(Me)N-O-TAGa (S15) (5.55 g, 3.6 mmol) was converted to 4-MePro-Val-Asn-Ala-(Me)N-O-TAGa (7) (4.76 g, quant.) as a white powder. [a] $]_{D}^{24.9}=-9.1\left(c 0.1, \mathrm{CHCl}_{3}\right)$; $\mathrm{mp} 100-102{ }^{\circ} \mathrm{C} ;{ }^{1} \mathrm{H}$ NMR $\left(500 \mathrm{MHz}, \mathrm{CDCl}_{3}\right.$, Signals were broad and complex due to the rotamers. Those derived from minor rotamers were not described here.) $\delta 8.31(1 \mathrm{H}, \mathrm{d}, J=8.5 \mathrm{~Hz}), 7.81(1 \mathrm{H}, \mathrm{br} \mathrm{s}), 7.61(1 \mathrm{H}, \mathrm{d}, J=6.5 \mathrm{~Hz}), 6.60(1 \mathrm{H}, \mathrm{br} \mathrm{s}), 6.57(2 \mathrm{H}, \mathrm{s})$, $5.88(1 \mathrm{H}$, br s), 4.91-4.74 $(4 \mathrm{H}$, br m, overlapped), $4.26(1 \mathrm{H}, \mathrm{br} \mathrm{m}), 4.00-3.91(6 \mathrm{H}, \mathrm{m}$, overlapped), $3.81(1 \mathrm{H}, \mathrm{br} \mathrm{d}, \mathrm{J}=9.0 \mathrm{~Hz}), 3.39$ $(1 \mathrm{H}, \mathrm{br} \mathrm{m}), 3.17(3 \mathrm{H}, \mathrm{s}), 3.08(1 \mathrm{H}, \mathrm{br} \mathrm{m}), 2.81-2.55(3 \mathrm{H}, \mathrm{br} \mathrm{m}$, overlapped), 2.30-2.03 (3H, br m, overlapped), 1.81-1.63 (6H, m, overlapped), 1.49-1.43 (6H, br m, overlapped), 1.35-1.22 (87H, br m, overlapped), $0.99(3 \mathrm{H}, \mathrm{d}, \mathrm{J}=6.0 \mathrm{~Hz}), 0.95(3 \mathrm{H}, \mathrm{d}, \mathrm{J}=6.5 \mathrm{~Hz})$, $0.91(3 \mathrm{H}, \mathrm{d}, J=7.0 \mathrm{~Hz}), 0.87(9 \mathrm{H}, \mathrm{t}, J=7.0 \mathrm{~Hz}) ;{ }^{13} \mathrm{C} \mathrm{NMR}\left(125 \mathrm{MHz}, \mathrm{CDCl}_{3}\right.$, Signals were broad and complex due to the rotamers. Those derived from minor rotamers were not described here.) $\delta 176.4,173.6,173.5,171.6,170.4,153.4,138.7,129.4,107.7,73.6$, $69.3,60.4,58.1,54.7,54.2,49.9,48.7,46.3,39.0,36.9,34.1,33.3,32.1,30.8,30.5,29.9-29.5$ (many signals overlapped), 26.3, 22.8, 19.6, 17.9, 17.58, 17.56, 14.2; IR (KBr) $v\left(\mathrm{~cm}^{-1}\right)$ 3270, 2916, 2849, 1635, 1468, 1236, 1121, 720; HRMS (FAB, NBA matrix) Calcd. for $\mathrm{C}_{80} \mathrm{H}_{149} \mathrm{O}_{9} \mathrm{~N}_{6}: 1338.1386\left([\mathrm{M}+\mathrm{H}]^{+}\right)$, Found: 1338.1384 .

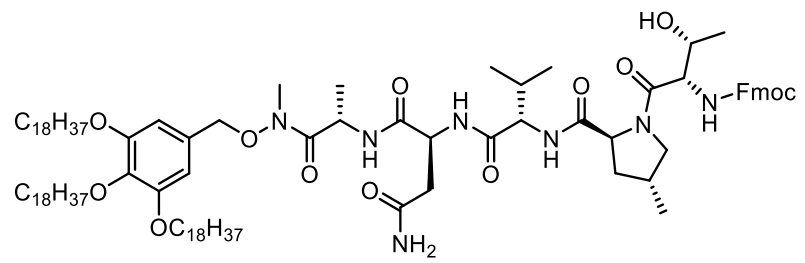

Fmoc-Thr-4-MePro-Val-Asn-Ala-(Me)N-O-TAGa (S16)

Following the general procedure described for condensation, 4-MePro-Val-Asn-Ala-(Me)N-O-TAGa (7) (4.70 g, $3.5 \mathrm{mmol})$ was converted to Fmoc-Thr-4-MePro-Val-Asn-Ala-(Me)N-O-TAGa (S16) $(5.84 \mathrm{~g} \text {, quant.) as a white powder. [a }]_{\mathrm{D}}^{25.0}=-22.8(\mathrm{c} 0.1, \mathrm{CHCl})_{3}$; $\mathrm{mp} 151-154{ }^{\circ} \mathrm{C} ;{ }^{1} \mathrm{H}$ NMR $\left(500 \mathrm{MHz}, \mathrm{CDCl}_{3}\right.$, Signals were broad and complex due to the rotamers. Those derived from minor rotamers were not described here.) $\delta 7.80-7.71(3 \mathrm{H}$, br m, overlapped), $7.59(2 \mathrm{H}$, br d, J=7.0 Hz), $7.37(2 \mathrm{H}$, br t, J = 7.5 Hz), $7.32-$ $7.28(3 \mathrm{H}$, br m, overlapped), $7.13(1 \mathrm{H}$, br d, $J=9.5 \mathrm{~Hz}), 6.95(1 \mathrm{H}, \mathrm{br} \mathrm{s}), 6.57(2 \mathrm{H}, \mathrm{s}), 6.31-6.22(2 \mathrm{H}$, br m, overlapped), 5.01-4.95 $(2 \mathrm{H}$, br m, overlapped), $4.84(1 \mathrm{H}, \mathrm{d}, J=10.5 \mathrm{~Hz}), 4.79(1 \mathrm{H}, \mathrm{d}, J=10.5 \mathrm{~Hz}), 4.70(1 \mathrm{H}, \mathrm{dd}, J=9.5,3.5 \mathrm{~Hz}), 4.63(1 \mathrm{H}, \mathrm{dd}, J=9.5,4.0$ $\mathrm{Hz}), 4.56(1 \mathrm{H}, \mathrm{br} \mathrm{m}), 4.46(1 \mathrm{H}, \mathrm{dd}, J=11.0,7.5 \mathrm{~Hz}), 4.35(1 \mathrm{H}, \mathrm{dd}, J=10.5,7.0 \mathrm{~Hz}), 4.30(1 \mathrm{H}, \mathrm{br} \mathrm{m}), 4.19(1 \mathrm{H}, \mathrm{t}, J=6.5 \mathrm{~Hz}), 4.03$ $(1 \mathrm{H}$, br t,$J=8.0 \mathrm{~Hz}), 3.97-3.92(6 \mathrm{H}, \mathrm{m}$, overlapped), $3.32(1 \mathrm{H}, \mathrm{brt}, J=9.0 \mathrm{~Hz}), 3.17(3 \mathrm{H}, \mathrm{s}), 2.81-2.69(2 \mathrm{H}$, br m, overlapped), 2.47 $(1 \mathrm{H}, \mathrm{br} \mathrm{m}), 2.24-2.16(2 \mathrm{H}$, br m, overlapped $), 1.93(1 \mathrm{H}, \mathrm{m}), 1.80-1.70(6 \mathrm{H}, \mathrm{m}$, overlapped $), 1.49-1.42(6 \mathrm{H}$, br m, overlapped), 1.35$1.22\left(90 \mathrm{H}\right.$, br m, overlapped), $1.09(3 \mathrm{H}, \mathrm{d}, J=6.0 \mathrm{~Hz}), 0.94(3 \mathrm{H}, \mathrm{d}, J=6.5 \mathrm{~Hz}), 0.87(12 \mathrm{H}$, app t, $J=7.0 \mathrm{~Hz}) ;{ }^{13} \mathrm{C} \mathrm{NMR}(125 \mathrm{MHz}$, $\mathrm{CDCl}_{3}$, Signals were broad and complex due to the rotamers. Those derived from minor rotamers were not described here.) $\delta 173.9$, 173.3, 172.2, 171.7, 171.3, 169.9, 156.4, 153.4, 143.9, 143.8, 141.4, 138.7, 129.5, 127.9, 127.2, 125.21, 125.16, 120.1, 107.7, 73.6, $69.3,68.0,67.0,61.5,58.1,57.0,54.6,50.5,47.3,46.2,42.3,37.8,37.1,34.2,33.0,32.1,31.0,30.5,29.9-29.5$ (many signals overlapped), 26.3, 23.6, 22.8, 19.5, 18.8, 18.0, 17.9, 17.4, 14.3; IR (KBr) v (cm-1) 3286, 2917, 2849, 1638, 1467, 1439, 1236, 1119, 720; HRMS (FAB, NBA + Nal matrix) Calcd. for $\mathrm{C}_{99} \mathrm{H}_{165} \mathrm{O}_{13} \mathrm{~N}_{7} \mathrm{Na}: 1683.2363\left([\mathrm{M}+\mathrm{Na}]^{+}\right)$, Found: 1683.2360.

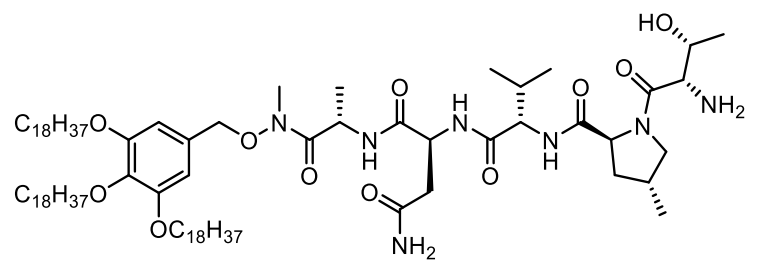

Thr-4-MePro-Val-Asn-Ala-(Me)N-O-TAGa (8)

Following the general procedure described for Fmoc deprotection, Fmoc-Thr-4-MePro-Val-Asn-Ala-(Me)N-O-TAGa (S16) (5.78 g, $3.5 \mathrm{mmol}$ ) was converted to Thr-4-MePro-Val-Asn-Ala-(Me)N-O-TAGa (8) (5.01 g, quant.) as a white powder. [a] $]_{D}^{25.1}=-35.3(c 0.1$, $\left.\mathrm{CHCl}_{3}\right)$; mp ca. $190{ }^{\circ} \mathrm{C}$ (decomp.); ${ }^{1} \mathrm{H}$ NMR $\left(500 \mathrm{MHz}, \mathrm{CDCl}_{3}\right.$, Signals were broad and complex due to the rotamers. Those derived from minor rotamers were not described here.) $\delta 7.34-7.30(3 \mathrm{H}, \mathrm{br} \mathrm{m}$, overlapped), $7.17(1 \mathrm{H}, \mathrm{br} \mathrm{s}), 6.81(1 \mathrm{H}, \mathrm{br} \mathrm{s}), 6.58(2 \mathrm{H}, \mathrm{s}), 4.97$ $(1 \mathrm{H}, \mathrm{br} \mathrm{m}), 4.93(1 \mathrm{H}, \mathrm{dd} J=9.5,4.5 \mathrm{~Hz}), 4.85(1 \mathrm{H}, \mathrm{d}, J=10.0 \mathrm{~Hz}), 4.79(1 \mathrm{H}, \mathrm{d}, J=10.5 \mathrm{~Hz}), 4.66-4.58(2 \mathrm{H}, \mathrm{br} \mathrm{m}), 4.11(1 \mathrm{H}, \mathrm{br} \mathrm{m})$, 3.98-3.92 $(6 \mathrm{H}, \mathrm{m}$, overlapped), $3.80(1 \mathrm{H}, \mathrm{br} \mathrm{m}), 3.30(1 \mathrm{H}, \mathrm{br} \mathrm{m}), 3.17(3 \mathrm{H}, \mathrm{s}), 3.16(1 \mathrm{H}, \mathrm{br} \mathrm{m}), 2.78-2.63(2 \mathrm{H}$, br m, overlapped), 2.46 $(1 \mathrm{H}, \mathrm{br} \mathrm{m}), 2.22(1 \mathrm{H}, \mathrm{m}), 2.12(2 \mathrm{H}, \mathrm{m}), 1.83(1 \mathrm{H}, \mathrm{m}$, overlapped $), 1.81-1.70(6 \mathrm{H}, \mathrm{m}$, overlapped $), 1.49-1.43(6 \mathrm{H}$, br m, overlapped), 1.37-1.22 (90H, br m, overlapped), $1.09(3 \mathrm{H}, \mathrm{d}, J=6.0 \mathrm{~Hz}), 0.94(3 \mathrm{H}, \mathrm{d}, J=7.0 \mathrm{~Hz}), 0.87(12 \mathrm{H}$, app t, $J=7.0 \mathrm{~Hz}) ;{ }^{13} \mathrm{C} \mathrm{NMR}(125$ 
$\mathrm{MHz}, \mathrm{CDCl}_{3}$, Signals were broad and complex due to the rotamers. Those derived from minor rotamers were not described here.) $\delta$ 174.2, 173.3 (two signals), 172.4, 171.5, 170.1, 153.4, 138.8, 129.4, 107.7, 73.6, 70.1, 69.3, 61.7, 57.7, 56.4, 54.5, 50.5, 46.0, 37.6, 37.2, 34.1, 32.9, 32.1, 30.5, 29.9-29.5 (many signals overlapped), 26.3, 22.8, 19.5, 18.0, 17.9, 17.5, 17.3, 14.2; IR $(\mathrm{KBr}) v\left(\mathrm{~cm}^{-1}\right)$ 3292, 2917, 2849, 1644, 1468, 1237, 1117, 720; HRMS (FAB, NBA matrix) Calcd. for $\mathrm{C}_{84} \mathrm{H}_{155} \mathrm{O}_{11} \mathrm{~N}_{7}: 1439.1863$ ([M + H] $]^{+}$), Found: 1439.1868.

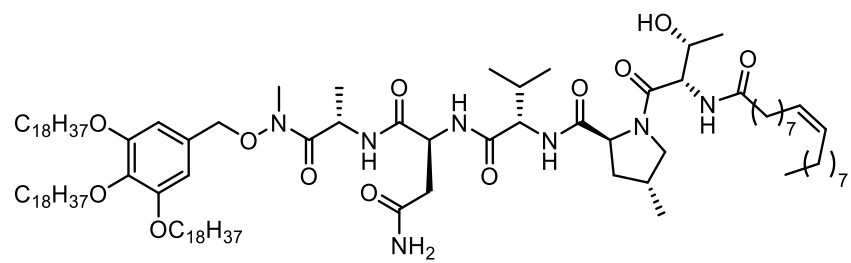

Oleic acid-Thr-4-MePro-Val-Asn-Ala-(Me)N-O-TAGa (9)

Following the general procedure described for condensation using oleic acid instead of Fmoc protected amino acid, Thr-4-MeProVal-Asn-Ala-(Me)N-O-TAGa (8) (2.97 g, $2.1 \mathrm{mmol}$ ) was converted to oleic acid-Thr-4-MePro-Val-Asn-Ala-(Me)N-O-TAGa (9) (3.52 g, quant.) as a white powder. Advanced Marfey's method for all the amino acid residues after complete acid hydrolysis in the same way as described above in "Screening of condensation reagents to suppress the epimerization in our previous report" section confirmed that no epimerization occurred. [a] $]_{\mathrm{D}}^{25.1}=-11.9\left(\mathrm{c} 0.1, \mathrm{CHCl}_{3}\right) ; \mathrm{mp} 151-152{ }^{\circ} \mathrm{C} ;{ }^{1} \mathrm{H} \mathrm{NMR}\left(500 \mathrm{MHz}, \mathrm{CDCl}_{3}\right) \delta 7.78(1 \mathrm{H}, \mathrm{br} \mathrm{d}, J=8.0$ $\mathrm{Hz}), 7.30(1 \mathrm{H}, \mathrm{br} \mathrm{d}, J=7.5 \mathrm{~Hz}), 7.09(1 \mathrm{H}, \mathrm{d}, J=9.5 \mathrm{~Hz}), 7.03(1 \mathrm{H}, \mathrm{br} \mathrm{s}), 6.77(1 \mathrm{H}, \mathrm{br} \mathrm{d}, J=8.5 \mathrm{~Hz}), 6.57(2 \mathrm{H}, \mathrm{s}), 6.37(1 \mathrm{H}, b r \mathrm{~s})$, 5.37-5.29 $(2 \mathrm{H}, \mathrm{m}$, overlapped), $4.98(1 \mathrm{H}, \mathrm{dd}, J=7.5,4.0 \mathrm{~Hz}), 4.95-4.89(2 \mathrm{H}, \mathrm{br} \mathrm{m}$, overlapped), $4.85(1 \mathrm{H}, \mathrm{d}, J=10.5 \mathrm{~Hz}), 4.80(1 \mathrm{H}$, $\mathrm{d}, J=10.5 \mathrm{~Hz}), 4.59(1 \mathrm{H}, \mathrm{dd}, J=9.0,4.0 \mathrm{~Hz}), 4.55(1 \mathrm{H}, \mathrm{dd}, J=9.0,6.5 \mathrm{~Hz}), 4.46(1 \mathrm{H}, \mathrm{d}, J=6.0 \mathrm{~Hz}), 4.29(1 \mathrm{H}, \mathrm{m}), 4.03(1 \mathrm{H}, \mathrm{dd}, J=$ 9.5, 7.5 Hz), 3.98-3.92 (6H, m, overlapped), $3.36(1 \mathrm{H}, \mathrm{brt}, J=9.5 \mathrm{~Hz}), 3.18(3 \mathrm{H}, \mathrm{s}), 2.79(1 \mathrm{H}, \mathrm{m}), 2.70(1 \mathrm{H}, \mathrm{m}), 2.46(1 \mathrm{H}, \mathrm{m}), 2.34$ $(2 \mathrm{H}, \mathrm{t}, J=8.0 \mathrm{~Hz}), 2.20-2.15(2 \mathrm{H}, \mathrm{br} \mathrm{m}$, overlapped), 2.20-1.98 $(3 \mathrm{H}, \mathrm{m}$, overlapped), $1.90(1 \mathrm{H}, \mathrm{m}), 1.82-1.72(6 \mathrm{H}, \mathrm{m}$, overlapped), 1.66-1.58 (2H, br m, overlapped), 1.49-1.43 (6H, br m, overlapped), 1.37-1.22 (110H, br m, overlapped), $1.09(3 \mathrm{H}, \mathrm{d}, J=7.0 \mathrm{~Hz})$, $0.94(3 \mathrm{H}, \mathrm{d}, J=6.5 \mathrm{~Hz}), 0.89-0.86\left(15 \mathrm{H}, \mathrm{m}\right.$, overlapped); ${ }^{13} \mathrm{C}$ NMR $\left(125 \mathrm{MHz}, \mathrm{CDCl}_{3}\right) \delta 173.8,173.4$ (two signals), $172.2,171.7$, 171.5, 169.9, 153.5, 138.8, 130.1, 129.9, 129.4, 107.7, 73.6, 69.4, 67.9, 61.5, 58.0, 54.7, 50.6, 46.2, 37.7, 37.1, 36.6, 34.1, 33.0, 32.1, 31.3, 30.5, 29.9-29.3 (many signals overlapped), 27.4, 27.3, 26.3, 25.7, 22.8, 19.5, 18.9, 17.93, 17.86, 17.3, 14.3; IR (KBr) v $\left(\mathrm{cm}^{-1}\right) 3279,2917,2849,1640,1438,1238,1121,719$; HRMS (FAB, NBA + Nal matrix) Calcd. for $\mathrm{C}_{102} \mathrm{H}_{187} \mathrm{O}_{12} \mathrm{~N}_{7} \mathrm{Na}: 1725.4135$ ([M + $\mathrm{Na}]^{+}$), Found: 1725.4131 .

\section{Reduction to afford the aldehyde}

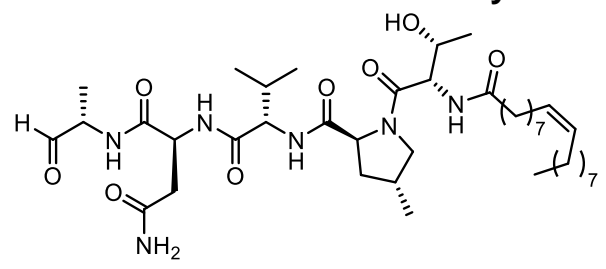

Oleic acid-Thr-4-MePro-Val-Asn-Ala-H (2)

To a solution of $9(850 \mathrm{mg}, 0.50 \mathrm{mmol}, 1.0 \mathrm{eq})$ in dehydrated THF $(50 \mathrm{~mL}, 0.01 \mathrm{M})$ was added dropwise $1.0 \mathrm{M} \mathrm{LiAlH}(\mathrm{Ot} \text { - } \mathrm{Bu})_{3}$ in dehydrated THF (4.99 mL, $10 \mathrm{eq}$, prepared by the procedure described in the literature. $\left.{ }^{[6]}\right)$ at room temperature. After stirring for $1 \mathrm{~h}$ at room temperature, the reaction mixture was then treated with aqueous $1 \mathrm{~N} \mathrm{HCl}(25 \mathrm{~mL})$ at $0{ }^{\circ} \mathrm{C}$ to quench the excess $\mathrm{LiAlH}(\mathrm{O}$ $\mathrm{Bu})_{3}$. After stirring for $10 \mathrm{~min}$ at room temperature, $\mathrm{MeOH}(250 \mathrm{~mL})$ was added. The resulting heterogeneous solution was stirred for a further $30 \mathrm{~min}$ at room temperature, and the precipitate was filtered and washed with additional $\mathrm{MeOH}$. The filtrate was roughly concentrated in vacuo, poured into a separatory funnel containing aqueous $1 \mathrm{~N} \mathrm{HCl}(20 \mathrm{~mL})$, and extracted with $\mathrm{CHCl}_{3}(3 \times 20 \mathrm{~mL})$. The combined organic extracts were dried with $\mathrm{Na}_{2} \mathrm{SO}_{4}$, filtered, and concentrated in vacuo. The residue was purified by flash column chromatography on silica gel $\left(\mathrm{CHCl}_{3} / \mathrm{MeOH}=50 / 1\right.$ to $10 / 1$ as eluent) to afford aldehyde $2(240.0 \mathrm{mg}, 63 \%)$ as a white powder and MePro-Thr amide bond-cleaved alcohol $\mathbf{S 1 7}(37.5 \mathrm{mg}, 20 \%$ yield) as a colorless oil (the structure is shown below).

$[\alpha]_{D}^{24.6}=-33.8$ (c 0.1, DMSO), $[\alpha]_{D}^{24.6}=-29.6\left(c 0.1, \mathrm{CHCl}_{3}\right) ; \mathrm{mp} 170-175^{\circ} \mathrm{C} ;{ }^{1} \mathrm{H}$ NMR $\left(500 \mathrm{MHz}\right.$, DMSO- $d_{6}$, Signals derived from the diastereomer of the a-position of alaninal moiety were not observed at all.) $\delta 9.34(1 \mathrm{H}, \mathrm{s}), 8.14(1 \mathrm{H}, \mathrm{d}, J=6.5 \mathrm{~Hz}), 8.11(1 \mathrm{H}, \mathrm{d}, J$ $=7.5 \mathrm{~Hz}), 7.87(2 \mathrm{H}$, app dd, $J=8.0,1.5 \mathrm{~Hz}), 7.37(1 \mathrm{H}, \mathrm{br} \mathrm{s}), 6.93(1 \mathrm{H}, \mathrm{br} \mathrm{s}), 5.35-5.29(2 \mathrm{H}, \mathrm{m}$, overlapped $), 4.67(1 \mathrm{H}, \mathrm{d}, J=6.0 \mathrm{~Hz})$, 4.55-4.47 (2H, m, overlapped), $4.41(1 \mathrm{H}, \mathrm{t}, J=7.5 \mathrm{~Hz}), 4.09-3.98(2 \mathrm{H}, \mathrm{m}$, overlapped), 3.83-3.76 (2H, m, overlapped), $3.27(1 \mathrm{H}, \mathrm{t}, J=$ $9.5 \mathrm{~Hz}), 2.55-2.46(2 \mathrm{H}, \mathrm{m}$, overlapped with solvent residual signals), $2.34(1 \mathrm{H}, \mathrm{m}), 2.17-2.06(2 \mathrm{H}, \mathrm{m}$, overlapped), $2.01-1.94(6 \mathrm{H}, \mathrm{m}$, overlapped), $1.65(1 \mathrm{H}, \mathrm{m}), 1.48-1.42(2 \mathrm{H}, \mathrm{m}$, overlapped), 1.32-1.19 $(20 \mathrm{H}, \mathrm{br} \mathrm{m}$, overlapped), $1.14(3 \mathrm{H}, \mathrm{d}, J=7.5 \mathrm{~Hz}), 1.08(3 \mathrm{H}, \mathrm{d}, J$ $=6.0 \mathrm{~Hz}), 0.97(3 \mathrm{H}, \mathrm{d}, J=7.0 \mathrm{~Hz}), 0.86-0.82\left(9 \mathrm{H}, \mathrm{m}\right.$, overlapped); ${ }^{13} \mathrm{C}$ NMR $\left(125 \mathrm{MHz}, \mathrm{DMSO}-d_{6}\right) \delta 201.4,172.2,171.9,171.4$, 171.3, 170.6, 169.5, 129.7, 66.9, 59.2, 58.0, 56.2, 54.0, 53.9, 49.5, 36.8, 36.5, 34.9, 32.0, 31.3, 30.4, 29.2-28.6 (many signals overlapped), 26.64, 26.60, 25.3, 22.1, 19.4, 19.1, 18.0, 17.2, 14.0, 13.6; IR (KBr) v(cm-1) 3285, 2924, 2853, 1736, 1639, 1542, 1426, 1235; HRMS (ESI+) Calcd. for $\mathrm{C}_{40} \mathrm{H}_{70} \mathrm{~N}_{6} \mathrm{O}_{8} \mathrm{Na:} 785.5153\left([\mathrm{M}+\mathrm{Na}]^{+}\right)$, Found: 785.5151 
MePro-Thr amide bond-cleaved alcohol S17

$[\alpha]_{\mathrm{D}}^{25.2}=-25.7\left(\mathrm{c} 0.1, \mathrm{CHCl}_{3}\right) ;{ }^{1} \mathrm{H}$ NMR $\left(500 \mathrm{MHz}\right.$, DMSO- $\left.d_{6}\right) \delta 7.24(1 \mathrm{H}, \mathrm{br} \mathrm{d}, J=9.0 \mathrm{~Hz}), 5.35-5.28(2 \mathrm{H}, \mathrm{m}$, overlapped), $4.55-$ $4.51(2 \mathrm{H}$, br m, overlapped), $3.85(1 \mathrm{H}, \mathrm{br} \mathrm{m}), 3.62(1 \mathrm{H}, \mathrm{m}), 3.42(1 \mathrm{H}, \mathrm{br} \mathrm{m}), 3.29(1 \mathrm{H}, \mathrm{br} \mathrm{m}), 2.15-2.04(2 \mathrm{H}, \mathrm{m}$, overlapped), $2.02-1.93$ (4H, br m, overlapped), 1.51-1.43 (2H, br m, overlapped), 1.30-1.20 $(20 \mathrm{H}, \mathrm{br} \mathrm{m}$, overlapped), $0.97(3 \mathrm{H}, \mathrm{d}, \mathrm{J}=6.0 \mathrm{~Hz}), 0.85(3 \mathrm{H}, \mathrm{t}, \mathrm{J}=$ $7.0 \mathrm{~Hz}) ;{ }^{13} \mathrm{C}$ NMR $\left(125 \mathrm{MHz}\right.$, DMSO- $\left.d_{6}\right) \delta 172.4,129.6,64.2,60.6,55.4,35.4,31.3,29.2-28.6$ (many signals overlapped), 26.64 , 26.60, 25.5, 22.1, 20.1, 14.0; IR (KBr) $v\left(\mathrm{~cm}^{-1}\right) 3294,2923,2853,1632,1541,1458,1065$; HRMS $\left(\mathrm{ESI}{ }^{+}\right) \mathrm{Calcd}_{\text {. for }} \mathrm{C}_{22} \mathrm{H}_{43} \mathrm{NO}_{3} \mathrm{Na}$ $392.3141\left([\mathrm{M}+\mathrm{Na}]^{+}\right)$, Found: 392.3139 .

\section{Recovery of benzyloxy methyl amine anchor molecule 3}

To a solution of $9(50.0 \mathrm{mg}, 0.029 \mathrm{mmol}, 1.0 \mathrm{eq})$ in dehydrated THF $(2.9 \mathrm{~mL}, 0.01 \mathrm{M})$ was added dropwise $1.0 \mathrm{M} \mathrm{LiAlH}(t-\mathrm{BuO})_{3}$ in dehydrated THF $\left(0.293 \mathrm{~mL}, 10 \mathrm{eq}\right.$, prepared by the procedure described in the literature. $\left.{ }^{[6]}\right)$ at room temperature. After stirring for $1 \mathrm{~h}$ at room temperature, the reaction mixture was then treated with aqueous $1 \mathrm{~N} \mathrm{HCl}(1.5 \mathrm{~mL})$ at $0{ }^{\circ} \mathrm{C}$ to quench the excess $\mathrm{LiAlH}(t-$ $\mathrm{BuO}_{3}$. After stirring for $10 \mathrm{~min}$ at room temperature, $\mathrm{MeOH}(14.7 \mathrm{~mL})$ was added. The resulting heterogeneous solution was stirred for a further $30 \mathrm{~min}$ at room temperature, and the precipitate was filtered and washed with additional $\mathrm{MeOH}$ to recover benzyloxy methyl amine anchor molecule 3 as its hydrogen chloride salt form (28.8 mg, quant.). Aldehyde 2 was obtained from the filtrate by the same procedure described above $(13.7 \mathrm{mg}, 61 \%)$. The ${ }^{1} \mathrm{H}$ NMR spectrum of this recovered 3 showed clear match with the data of newly prepared 3 in $\mathrm{CDCl}_{3}+$ small amount of conc. $\mathrm{HCl}$ (see NMR spectra section below).

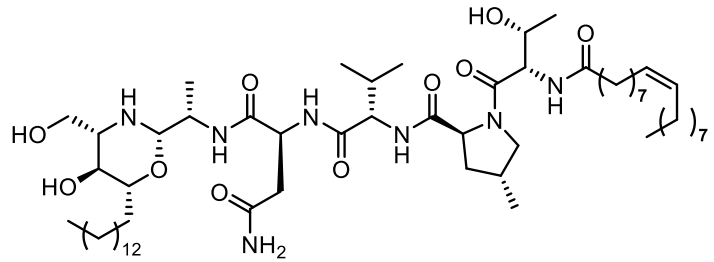
Kozupeptin A (1)

To a solution of $2(17.5 \mathrm{mg}, 0.023 \mathrm{mmol}, 1.0 \mathrm{eq})$ in $\mathrm{CHCl}_{3}(1.2 \mathrm{~mL})$ was added phytosphingosine $(8.7 \mathrm{mg}, 0.028 \mathrm{mmol}, 1.2 \mathrm{eq})$ at room temperature. After stirring for $6 \mathrm{~h}$ at room temperature, the reaction mixture was concentrated in vacuo. The residue was purified by flash column chromatography on silica gel $\left(\mathrm{CHCl}_{3} / \mathrm{MeOH}\right)$ to afford kozupeptin $\mathrm{A}(\mathbf{1})(23.1 \mathrm{mg}, 95 \%)$ as a white powder. All physical data for 1 obtained here matched with the data in our previous paper. ${ }^{[2]}$ In ${ }^{1} \mathrm{H}$ NMR spectrum, signals derived from the diastereomer of the a-position of alaninal moiety were not observed at all. The amide-H region was shown below (upper: derived from this method, lower: derived from the previous report method using HBTU as a condensation reagent to get Weinreb amide S2).

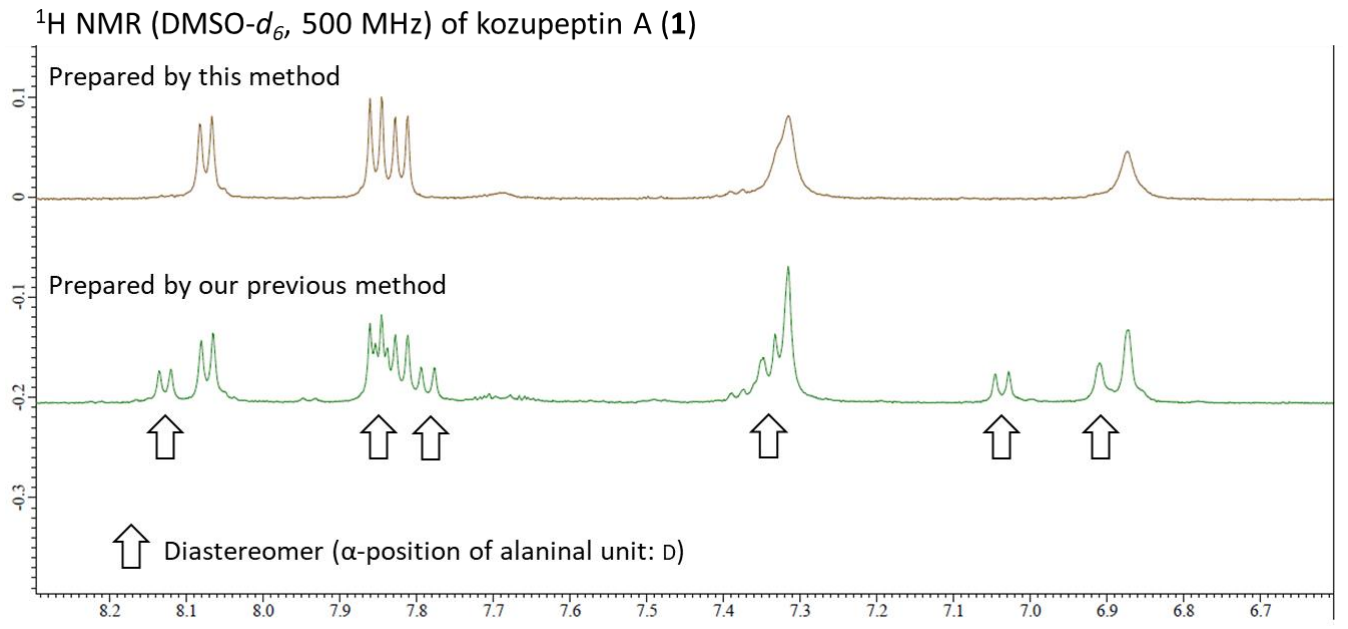




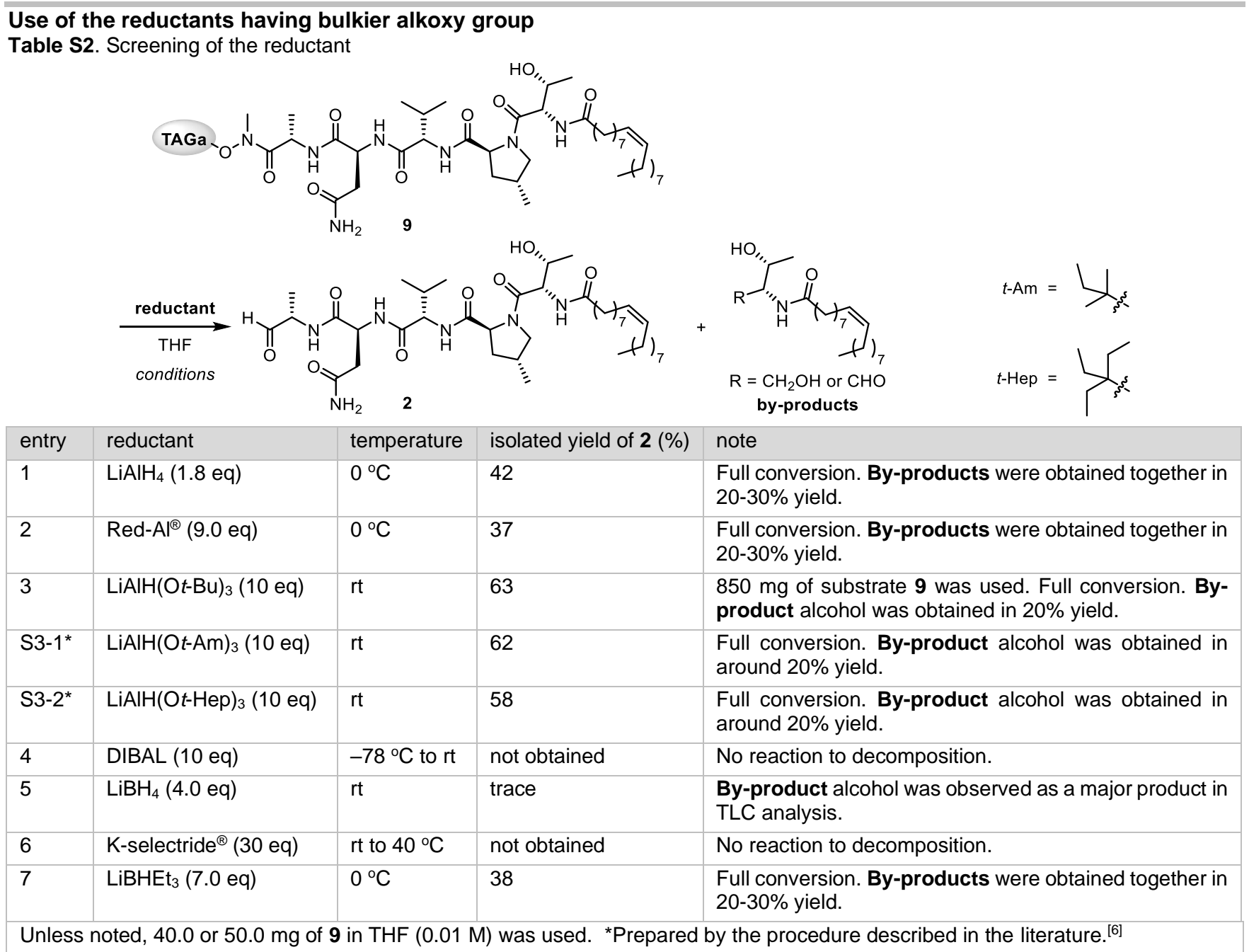

\section{Use of a model substrate}

Scheme S3. Use of a model substrate without Thr-Pro amide bond
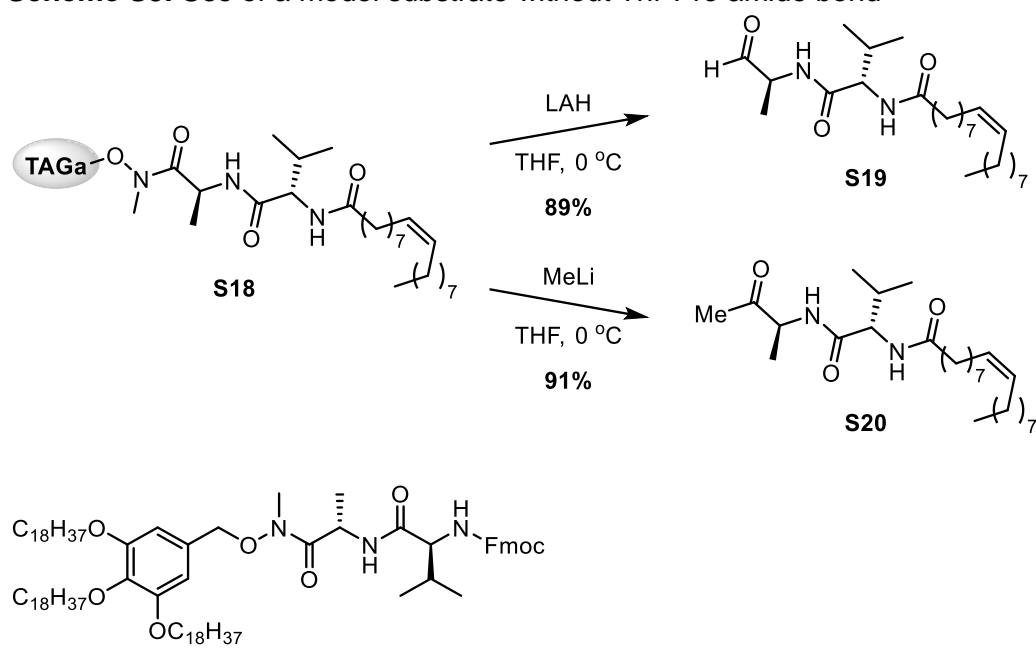

Fmoc-Val-Ala-(Me)N-O-TAGa (S21)

Following the general procedure described for condensation, Ala-(Me)N-O-TAGa (4) $(817 \mathrm{mg}, 0.81 \mathrm{mmol})$ was converted to Fmoc-Val-Ala-(Me)N-O-TAGa (S21) $(1.08 \mathrm{~g} \text {, quant.) as a white powder. [a }]_{\mathrm{D}}^{24.3}=-13.8\left(\mathrm{c} 0.1, \mathrm{CHCl}_{3}\right) ; \mathrm{mp} 65-69{ }^{\circ} \mathrm{C} ;{ }^{1} \mathrm{H} \mathrm{NMR}(500$ $\left.\mathrm{MHz}, \mathrm{CDCl}_{3}\right) \delta 7.76(2 \mathrm{H}, \mathrm{d}, J=7.5 \mathrm{~Hz}), 7.61(2 \mathrm{H}, \mathrm{br} \mathrm{dd}, J=7.5,4.0 \mathrm{~Hz}), 7.39(2 \mathrm{H}, \mathrm{br} \mathrm{td}, J=7.5,2.0 \mathrm{~Hz}), 7.31(2 \mathrm{H}, \mathrm{m}), 6.63(1 \mathrm{H}, \mathrm{br}$ s, overlapped), $6.60(2 \mathrm{H}, \mathrm{s}), 5.43(1 \mathrm{H}, \mathrm{br} \mathrm{d}, J=9.0 \mathrm{~Hz}), 5.05(1 \mathrm{H}, \mathrm{br} \mathrm{m}), 4.85(2 \mathrm{H}, \mathrm{br} \mathrm{s}), 4.45(1 \mathrm{H}, \mathrm{dd}, J=10.5,7.5 \mathrm{~Hz}), 4.36(1 \mathrm{H}, \mathrm{dd}$ $J=11.0,6.5 \mathrm{~Hz}), 4.23(1 \mathrm{H}, \mathrm{t}, J=7.0 \mathrm{~Hz}), 4.07(1 \mathrm{H}, \mathrm{br} \mathrm{dd}, J=8.5,6.5 \mathrm{~Hz}), 3.99-3.94(6 \mathrm{H}, \mathrm{m}$, overlapped), $3.21(3 \mathrm{H}, \mathrm{s}), 2.13(1 \mathrm{H}, \mathrm{m})$, 1.82-1.71 (6H, m, overlapped), 1.50-1.44 (6H, br m, overlapped), 1.36-1.22 (87H, br m, overlapped), $0.97(3 \mathrm{H}, \mathrm{d}, \mathrm{J}=6.5 \mathrm{~Hz}), 0.94$ $(3 \mathrm{H}, \mathrm{d}, J=7.0 \mathrm{~Hz}), 0.88(9 \mathrm{H}, \mathrm{t}, J=7.5 \mathrm{~Hz}) ;{ }^{13} \mathrm{C} \mathrm{NMR}\left(125 \mathrm{MHz}, \mathrm{CDCl}_{3}\right.$, Signals were complex due to the rotamers.) $\delta 173.4,170.8$, 156.4, 153.5, 144.1, 143.9, 141.4, 138.9, 129.1, 127.8, 127.2, 125.3, 125.2, 120.11, 120.09, 107.8, 77.6, 73.6, 69.3, 67.2, 60.3, 47.3, 46.0, 34.1, 32.1, 31.7, 30.5, 29.9-29.5 (many signals overlapped), 26.3, 22.8, 19.3, 18.3, 17.9, 14.3; IR $(\mathrm{KBr}) v\left(\mathrm{~cm}^{-1}\right) 3290,2916$, 


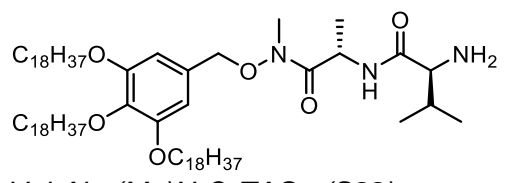

Val-Ala-(Me)N-O-TAGa (S22)

Following the general procedure described for Fmoc deprotection, Fmoc-Val-Ala-(Me)N-O-TAGa (S21) (965 mg, $0.72 \mathrm{mmol})$ was converted to Val-Ala-(Me)N-O-TAGa (S22) $(804 \mathrm{mg} \text {, quant.) as a white powder. [a] }]_{\mathrm{D}}^{24.4}=-10.0\left(\mathrm{c} 0.1, \mathrm{CHCl}_{3}\right) ; \mathrm{mp}^{58-59}{ }^{\circ} \mathrm{C} ;{ }^{1} \mathrm{H} \mathrm{NMR}$ $\left(500 \mathrm{MHz}, \mathrm{CDCl}_{3}\right) \delta 7.82(1 \mathrm{H}, \mathrm{br} \mathrm{d}, J=8.0 \mathrm{~Hz}), 6.62(2 \mathrm{H}, \mathrm{s}), 5.10(1 \mathrm{H}, \mathrm{br} \mathrm{m}), 4.89(1 \mathrm{H}, \mathrm{d}, J=10.0 \mathrm{~Hz}), 4.84(1 \mathrm{H}, \mathrm{d}, J=10.5 \mathrm{~Hz})$, 4.01-3.91 (6H, m, overlapped), $3.25(1 \mathrm{H}, \mathrm{d}, J=4.0 \mathrm{~Hz}), 3.20(3 \mathrm{H}, \mathrm{s}), 2.26(1 \mathrm{H}, \mathrm{m}), 1.82-1.70(6 \mathrm{H}, \mathrm{br} \mathrm{m}$, overlapped $), 1.49-1.43(6 \mathrm{H}$, br m, overlapped), $1.36-1.22$ (87H, br m, overlapped), $1.00(3 \mathrm{H}, \mathrm{d}, J=7.0 \mathrm{~Hz}), 0.89-0.85\left(12 \mathrm{H}, \mathrm{m}\right.$, overlapped); ${ }^{13} \mathrm{C} \mathrm{NMR}(125 \mathrm{MHz}$ $\mathrm{CDCl}_{3}$, Signals were complex due to the rotamers.) $\delta 173.94,173.87,153.5,138.8,129.3,107.9,77.7,73.6,69.3,60.3,45.3,34.0$, 32.1, 31.2, 30.5, 29.9-29.5 (many signals overlapped), 26.3, 22.8, 19.8, 18.5, 16.4, 14.3; IR (KBr) $v\left(\mathrm{~cm}^{-1}\right) 2916,2849,1659,1467$, 1236, 1119, 720; HRMS (FAB, NBA matrix) Calcd. for $\mathrm{C}_{70} \mathrm{H}_{134} \mathrm{O}_{6} \mathrm{~N}_{3}: 1113.0273\left([\mathrm{M}+\mathrm{H}]^{+}\right)$, Found: 1113.0282.<smiles>CCCCCCCCCCCCC(=O)NC(C(=O)N[C@@H](C)C(=O)N(C)OCc1cc(OCC)c(OCC)c(OCC)c1)C(C)C</smiles>

Oleic acid-Val-Ala-(Me)N-O-TAGa (S18)

Following the general procedure described for condensation using oleic acid instead of Fmoc protected amino acid, Val-Ala(Me)N-O-TAGa (S22) $(770 \mathrm{mg}, 0.72 \mathrm{mmol}$ ) was converted to oleic acid-Val-Ala-(Me)N-O-TAGa (S18) $(933 \mathrm{mg}, 98 \%)$ as a white powder. $[\alpha]_{D}^{24.4}=-11.6\left(c 0.1, \mathrm{CHCl}_{3}\right) ; \mathrm{mp} 54-55^{\circ} \mathrm{C} ;{ }^{1} \mathrm{H} \mathrm{NMR}\left(500 \mathrm{MHz}, \mathrm{CDCl}_{3}\right) \delta 6.70(1 \mathrm{H}, \mathrm{br} \mathrm{d}, J=7.0 \mathrm{~Hz}), 6.59(2 \mathrm{H}, \mathrm{s}), 6.11(1 \mathrm{H}$, br d, J = 7.5 Hz), 5.36-5.29 (2H, m, overlapped), $5.01(1 \mathrm{H}, \mathrm{br} \mathrm{m}), 4.83(2 \mathrm{H}, \mathrm{s}), 4.36(1 \mathrm{H}, \mathrm{dd}, J=8.5,6.5 \mathrm{~Hz}), 3.99-3.93(6 \mathrm{H}, \mathrm{m}$, overlapped), $3.20(3 \mathrm{H}, \mathrm{s}), 2.22(2 \mathrm{H}, \mathrm{m}), 2.07(1 \mathrm{H}, \mathrm{m}), 2.01-1.98(3 \mathrm{H}, \mathrm{br} \mathrm{m}$, overlapped), 1.82-1.70 (6H, m, overlapped), 1.68-1.59 (2H, br m, overlapped), 1.49-1.43 (6H, br m, overlapped), 1.36-1.22 (107H, br m, overlapped), $0.94(3 \mathrm{H}, \mathrm{d}, J=7.0 \mathrm{~Hz}), 0.92(3 \mathrm{H}, \mathrm{d}, \mathrm{J}$ $=6.5 \mathrm{~Hz}), 0.87(12 \mathrm{H}, \mathrm{t}, J=7.0 \mathrm{~Hz}) ;{ }^{13} \mathrm{C} \mathrm{NMR}\left(125 \mathrm{MHz}, \mathrm{CDCl}_{3}\right.$, Signals were complex because of the rotamers.) $\delta 173.4,173.2$, $170.8,153.5,138.9,130.1,129.9,129.2,107.8,77.6,73.6,69.3,58.0,45.9,36.9,34.1,32.1,32.0,31.7,30.5$, $29.9-29.3$ (many signals overlapped), 27.34, 27.30, 26.3, 25.9, 22.8, 19.3, 18.19, 18.16, 14.2; IR (KBr) v( $\left.\mathrm{cm}^{-1}\right) 3303,2917,2849,1638,1467,1235$, 1118, 721; HRMS (FAB, NBA + Nal matrix) Calcd. for $\mathrm{C}_{88} \mathrm{H}_{165} \mathrm{~N}_{3} \mathrm{O}_{7} \mathrm{Na}: 1399.2545\left([\mathrm{M}+\mathrm{Na}]^{+}\right)$, Found: 1399.2542.

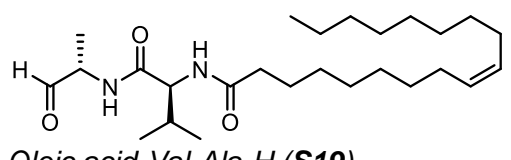

Oleic acid-Val-Ala-H (S19)

To a solution of $\mathbf{S} 18(20.0 \mathrm{mg}, 0.015 \mathrm{mmol}, 1.0 \mathrm{eq})$ in dehydrated THF $(1.8 \mathrm{~mL}, 0.008 \mathrm{M})$ was added dropwise $2.0 \mathrm{M} \mathrm{LiAlH}_{4}$ in dehydrated THF $(8.7 \mu \mathrm{L}, 1.2 \mathrm{eq})$ at $0{ }^{\circ} \mathrm{C}$. After stirring for $15 \mathrm{~min}$ at $0{ }^{\circ} \mathrm{C}$, the reaction mixture was then treated with aqueous $1 \mathrm{~N} \mathrm{HCl}$ $(0.9 \mathrm{~mL})$ at $0{ }^{\circ} \mathrm{C}$ to quench the excess $\mathrm{LiAlH}_{4}$. After stirring for $10 \mathrm{~min}$ at room temperature, $\mathrm{MeOH}(9.0 \mathrm{~mL})$ was added. The resulting heterogeneous solution was stirred for a further $30 \mathrm{~min}$ at room temperature, and the precipitate was filtered and washed with additional $\mathrm{MeOH}$. The filtrate was roughly concentrated in vacuo, poured into a separatory funnel containing aqueous $1 \mathrm{~N} \mathrm{HCl}(10$ $\mathrm{mL})$, and extracted with $\mathrm{CHCl}_{3}(3 \times 10 \mathrm{~mL})$. The combined organic extracts were dried with $\mathrm{Na}_{2} \mathrm{SO}_{4}$, filtered, and concentrated in vacuo. The residue was purified by flash column chromatography on silica gel $\left(\mathrm{CHCl}_{3} / \mathrm{MeOH}=200 / 1\right.$ to $20 / 1$ as eluent) to afford aldehyde $\mathbf{S} 19(5.6 \mathrm{mg}, 89 \%)$ as a white powder. [a $]_{\mathrm{D}}^{24.5}=-12.5\left(\mathrm{c} 0.1, \mathrm{CHCl}_{3}\right) ; \mathrm{mp} 96-99^{\circ} \mathrm{C} ;{ }^{1} \mathrm{H} \mathrm{NMR}(500 \mathrm{MHz}, 500 \mathrm{MHz}, \mathrm{DMSO}$ $d_{6}$, Signals were broad and complex due to the rotamers. Those derived from minor rotamers were not described here.) $\delta 9.37(1 \mathrm{H}$, s), $8.45(1 \mathrm{H}, \mathrm{d}, J=6.0 \mathrm{~Hz}), 7.83(1 \mathrm{H}, \mathrm{d}, J=7.5 \mathrm{~Hz}), 5.35-5.29(2 \mathrm{H}, \mathrm{m}$, overlapped $), 4.19(1 \mathrm{H}, \mathrm{m}), 4.07(1 \mathrm{H}, \mathrm{m}), 2.21-2.07(2 \mathrm{H}, \mathrm{m}$, overlapped), 1.99-1.91 (5H, m, overlapped), 1.53-1.42 (2H, m, overlapped), 1.30-1.22 (20H, br m, overlapped), $1.16(3 \mathrm{H}, \mathrm{d}, J=7.5$ $\mathrm{Hz}), 0.88-0.84\left(9 \mathrm{H}, \mathrm{m}\right.$, overlapped); ${ }^{13} \mathrm{C}$ NMR $\left(125 \mathrm{MHz}\right.$, DMSO- $d_{6}$, Signals were broad and complex due to the rotamers. Those derived from minor rotamers were not described here.) $\delta$ 201.9, 173.2, 172.5, 130.5, 58.2, 54.7, 36.1, 32.2, 31.3, $30.0-29.5$ (many signals overlapped), 27.53, 27.49, 26.3, 23.0, 20.1, 19.1, 14.9, 14.4; IR $(\mathrm{KBr}) v\left(\mathrm{~cm}^{-1}\right) 3284,2919,2850,1733,1633,1541,1466$, 1386, 693; HRMS (FAB, NBA matrix) Calcd. for $\mathrm{C}_{26} \mathrm{H}_{49} \mathrm{O}_{3} \mathrm{~N}_{2}: 437.3743\left([\mathrm{M}+\mathrm{H}]^{+}\right)$, Found: 437.3747.

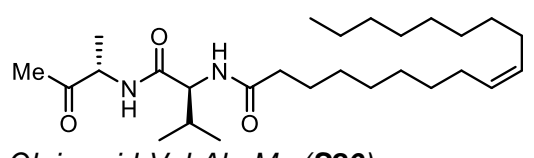

Oleic acid-Val-Ala-Me (S20)

To a solution of $\mathbf{S} 18(20.0 \mathrm{mg}, 0.015 \mathrm{mmol}, 1.0 \mathrm{eq})$ in dehydrated THF (1.8 mL, $0.008 \mathrm{M})$ was added dropwise $1.11 \mathrm{M} \mathrm{MeLi}$ in dehydrated diethyl ether $\left(\mathrm{Et}_{2} \mathrm{O}\right)(131 \mu \mathrm{L}, 10 \mathrm{eq})$ at $0{ }^{\circ} \mathrm{C}$. After stirring for $20 \mathrm{~min}$ at $0{ }^{\circ} \mathrm{C}$, the reaction mixture was then treated with aqueous $1 \mathrm{~N} \mathrm{HCl}(0.9 \mathrm{~mL})$ at $0{ }^{\circ} \mathrm{C}$ to quench the excess MeLi. After stirring for 10 min at room temperature, $\mathrm{MeOH}(9.0 \mathrm{~mL})$ was added. The resulting heterogeneous solution was stirred for a further $30 \mathrm{~min}$ at room temperature, and the precipitate was filtered and washed with additional $\mathrm{MeOH}$. The filtrate was roughly concentrated in vacuo, poured into a separatory funnel containing aqueous $1 \mathrm{~N} \mathrm{HCl}(10 \mathrm{~mL})$, and extracted with $\mathrm{CHCl}_{3}(3 \times 10 \mathrm{~mL})$. The combined organic extracts were dried with $\mathrm{Na}_{2} \mathrm{SO}_{4}$, filtered, and concentrated in vacuo. The residue was purified by PLC on silica gel $\left(\mathrm{CHCl}_{3} / \mathrm{MeOH}=10 / 1\right)$ to afford methyl ketone S20 (5.9 mg, $91 \%)$ as a white powder. $[\alpha]_{D}{ }_{D}^{24.5}=-9.0\left(c 0.1, \mathrm{CHCl}_{3}\right) ; \mathrm{mp} 93-97^{\circ} \mathrm{C} ;{ }^{1} \mathrm{H}$ NMR $\left(500 \mathrm{MHz}\right.$, DMSO- $d_{6}$, Signals were broad and complex due to the rotamers.) $\delta 8.34-8.30(1 \mathrm{H}, \mathrm{m}), 7.84-7.79(1 \mathrm{H}, \mathrm{m}), 5.35-5.29(2 \mathrm{H}, \mathrm{m}$, overlapped), 4.23-4.11 (2H, m, overlapped), 2.20$2.07(2 \mathrm{H}, \mathrm{m}$, overlapped), 2.06 \& $2.04(3 \mathrm{H}$, two s) 1.99-1.90 (5H, m, overlapped), 1.51-1.42 (2H, br m, overlapped), 1.30-1.22 (20H, br m, overlapped), $1.15(3 \mathrm{H}, \mathrm{t}, J=7.0 \mathrm{~Hz}), 0.87-0.83\left(9 \mathrm{H}, \mathrm{m}\right.$, overlapped); ${ }^{13} \mathrm{C}$ NMR $\left(125 \mathrm{MHz}\right.$, DMSO- $d_{6}$, Signals were complex due 
to the rotamers) $\delta 208.0,207.4,172.3,172.2,171.3,171.1,129.6,57.7,57.3,54.1,54.0,35.1,31.3,30.4,30.2,29.1-28.6$ (many signals overlapped), 26.61, 26.57, 26.1, 25.7, 25.41, 25.38, 22.1, 19.24, 19.19, 18.4, 18.1, 15.8, 15.6, 14.0; IR (KBr) $v\left(\mathrm{~cm}^{-1}\right) 3284$, 2921, 2851, 1720, 1633, 1540, 1467, 1386, 719; HRMS (ESI+) Calcd. for $\mathrm{C}_{27} \mathrm{H}_{50} \mathrm{~N}_{2} \mathrm{O}_{3} \mathrm{Na}: 473.3719$ ([M + Na] $]^{+}$), Found:473.3720.

\section{Using TAGb-type anchor molecule}<smiles>CCOc1ccc(CN2C(=O)[C@@H](NC(C)=O)[C@H]3CCCCC32)c(OCC)c1</smiles>

\section{Fmoc-Ala-(Me)N-O-TAGb (S23)}

Following the general procedure described for condensation, Me-HN-O-TAGb (S11) $(1.50 \mathrm{~g}, 1.9 \mathrm{mmol})$ was converted to FmocAla-(Me)N-O-TAGb (S23) $(2.03 \mathrm{~g}, 99 \%)$ as a white powder. $\left.[\alpha]_{D}{ }^{24.3}=23.6\left(c 0.1, \mathrm{CHCl}_{3}\right) ; \mathrm{mp} 53-54{ }^{\circ} \mathrm{C} ;{ }^{1} \mathrm{H} \mathrm{NMR}(500 \mathrm{MHz}, \mathrm{CDCl})\right) \delta$ $7.76(2 \mathrm{H}, \mathrm{d}, J=7.5 \mathrm{~Hz}), 7.62(2 \mathrm{H}, \mathrm{d}, J=7.0 \mathrm{~Hz}), 7.40(2 \mathrm{H}, \mathrm{t}, J=7.5 \mathrm{~Hz}), 7.32(2 \mathrm{H}, \mathrm{t}, J=7.5 \mathrm{~Hz}), 7.24(1 \mathrm{H}, \mathrm{br} \mathrm{d}, J=8.0 \mathrm{~Hz}), 6.45-$ $6.43(2 \mathrm{H}$, br m, overlapped), $5.59(1 \mathrm{H}$, br d, $J=8.5 \mathrm{~Hz}), 4.97(1 \mathrm{H}, \mathrm{d}, J=9.5 \mathrm{~Hz}), 4.88-4.84(2 \mathrm{H}$, br m, overlapped), 4.39-4.31 (2H, m, overlapped), $4.23(1 \mathrm{H}, \mathrm{t}, J=7.5 \mathrm{~Hz}), 4.04-3.95(2 \mathrm{H}, \mathrm{br} \mathrm{m}$, overlapped), $3.91(2 \mathrm{H}, \mathrm{t}, J=7.0 \mathrm{~Hz}), 3.27(3 \mathrm{H}, \mathrm{s}), 1.82(2 \mathrm{H}, \mathrm{m}), 1.73(2 \mathrm{H}$, $\mathrm{m}), 1.48-1.38\left(4 \mathrm{H}\right.$, br m, overlapped), 1.34-1.22 $\left(75 \mathrm{H}\right.$, br m, overlapped), $0.88(6 \mathrm{H}, \mathrm{t}, J=7.0 \mathrm{~Hz}) ;{ }^{13} \mathrm{C} \mathrm{NMR}\left(125 \mathrm{MHz}, \mathrm{CDCl}_{3}, \mathrm{Signals}\right.$ were complex due to the rotamers) $\delta 173.7,161.7,159.1,155.7,144.2,144.0,141.43,141.40,133.0,127.8,127.2,125.4,125.3$, 120.1, 114.6, 104.9, 99.9, 71.6, 68.33, 68.27, 67.0, 47.4, 47.3, 33.5, 32.1, 29.9-29.3 (many signals overlapped), 26.23, 26.17, 22.8, 18.8, 14.3; IR $(\mathrm{KBr}) \vee\left(\mathrm{cm}^{-1}\right) 3315,2916,2849,1717,1669,1468,1253,1178,1131,1034,739$; HRMS (FAB, NBA + Nal matrix) Calcd. for $\mathrm{C}_{70} \mathrm{H}_{114} \mathrm{~N}_{2} \mathrm{O}_{6} \mathrm{Na:} 1101.8575\left([\mathrm{M}+\mathrm{Na}]^{+}\right)$, Found: 1101.8588 .<smiles>COc1ccc(CON(C)C(=O)[C@H](C)N)c(OCCNCCOc2ccccc2)c1</smiles>

\section{Ala-(Me)N-O-TAGb (S24)}

Following the general procedure described for Fmoc deprotection, Fmoc-Ala-(Me)N-O-TAGb (S23) (1.67 g, $1.6 \mathrm{mmol})$ was

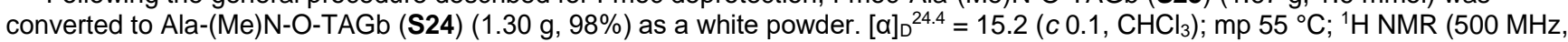
$\left.\mathrm{CDCl}_{3}\right) \delta 7.16(1 \mathrm{H}, \mathrm{d}, J=8.0 \mathrm{~Hz}), 6.45-6.42(2 \mathrm{H}, \mathrm{m}$, overlapped), $4.86(1 \mathrm{H}, \mathrm{d}, J=10.0 \mathrm{~Hz}), 4.77(1 \mathrm{H}, \mathrm{d}, J=9.5 \mathrm{~Hz}), 3.98-3.92(5 \mathrm{H}$, $\mathrm{m}$, overlapped), $3.25(3 \mathrm{H}, \mathrm{s}), 1.84-1.73(4 \mathrm{H}, \mathrm{m}$, overlapped), 1.48-1.41 (4H, br m, overlapped), 1.35-1.20 (75H, br m, overlapped), $0.87(6 \mathrm{H}, \mathrm{t}, J=7.0 \mathrm{~Hz}) ;{ }^{13} \mathrm{C}$ NMR $\left(125 \mathrm{MHz}, \mathrm{CDCl}_{3}\right) \delta 177.6,161.7,159.1,132.9,114.8,104.9,99.8,71.0,68.34,68.30,46.8,33.4$, 32.1, 29.9-29.3 (many signals overlapped), 26.24, 26.17, 22.8, 20.4, 14.3; IR $(\mathrm{KBr}) v\left(\mathrm{~cm}^{-1}\right) 2915,2849,1658,1614,1470,1181$, 1131, 718; HRMS (FAB, NBA + Nal matrix) Calcd. for $\mathrm{C}_{55} \mathrm{H}_{104} \mathrm{~N}_{2} \mathrm{O}_{4} \mathrm{Na}: 879.7894\left([\mathrm{M}+\mathrm{Na}]^{+}\right)$, Found: 879.7898.<smiles>CCCNC(CC(N)=O)C(=O)N[C@@H](C)C(=O)N(C)OCc1ccc(OCC)cc1OCC</smiles>

Fmoc-Asn-Ala-(Me)N-O-TAGb (S25)

Following the general procedure described for condensation, Ala-(Me)N-O-TAGb (S24) (1.25 g, $1.5 \mathrm{mmol})$ was converted to Fmoc-Asn-Ala-(Me)N-O-TAGb (S25) (1.74 g, quant.) as a white powder. [a] $]^{24.4}=20.5\left(\mathrm{c} 0.1, \mathrm{CHCl}_{3}\right) ; \mathrm{mp} 75-79^{\circ} \mathrm{C} ;{ }^{1} \mathrm{H} \mathrm{NMR}(500$ $\left.\mathrm{MHz}, \mathrm{CDCl}_{3}\right) \delta 7.75(2 \mathrm{H}, \mathrm{d}, J=7.5 \mathrm{~Hz}), 7.60(2 \mathrm{H}, \mathrm{br} \mathrm{dd}, J=7.0,4.5 \mathrm{~Hz}), 7.53(1 \mathrm{H}, \mathrm{br} \mathrm{d}, J=5.0 \mathrm{~Hz}), 7.39(2 \mathrm{H}, \mathrm{t}, J=7.5 \mathrm{~Hz}), 7.31$ $(2 \mathrm{H}, \mathrm{t}, J=7.5 \mathrm{~Hz}), 7.23(1 \mathrm{H}, \mathrm{br} \mathrm{d}, J=8.0 \mathrm{~Hz}), 6.44-6.42(2 \mathrm{H}$, overlapped), $6.38(1 \mathrm{H}, \mathrm{br} \mathrm{d}, J=7.5 \mathrm{~Hz}), 6.13(1 \mathrm{H}, \mathrm{br} \mathrm{s}), 5.65(1 \mathrm{H}, \mathrm{br} \mathrm{s})$, 5.01-4.96 $(2 \mathrm{H}, \mathrm{br} \mathrm{m}$, overlapped), $4.85(1 \mathrm{H}, \mathrm{d}, J=9.0 \mathrm{~Hz}), 4.61(1 \mathrm{H}, \mathrm{br} \mathrm{m}), 4.41-4.34(2 \mathrm{H}, \mathrm{br} \mathrm{m}$, overlapped $), 4.22(1 \mathrm{H}, \mathrm{t}, J=7.5 \mathrm{~Hz})$, 4.02-3.92 (4H, m, overlapped), $3.24(3 \mathrm{H}, \mathrm{s}), 2.92(1 \mathrm{H}, \mathrm{br} \mathrm{m}), 2.65(1 \mathrm{H}, \mathrm{br} \mathrm{m}), 1.81-1.74(4 \mathrm{H}, \mathrm{br} \mathrm{m}$, overlapped), 1.47-1.41 $(4 \mathrm{H}, \mathrm{br} \mathrm{m}$, overlapped), $1.35-1.22\left(75 \mathrm{H}\right.$, br m, overlapped), $0.88(6 \mathrm{H}, \mathrm{t}, J=7.5 \mathrm{~Hz}) ;{ }^{13} \mathrm{C} \mathrm{NMR}\left(125 \mathrm{MHz}, \mathrm{CDCl}_{3}\right.$, Signals were complex due to the rotamers) $\delta 173.4,173.3,170.3,161.7,159.1,156.2,144.0,143.9,141.4,133.0,127.8,127.2,125.3,120.1,114.7,104.9,99.9$, 71.6, 68.3, 67.4, 51.3, 47.2, 46.5, 37.8, 33.5, 32.1, 29.9-29.3 (many signals overlapped), 26.2, 22.8, 17.8, 14.3; IR (KBr) $v\left(\mathrm{~cm}^{-1}\right)$ $3421,2917,2849,1661,1469,1265,1178,1130,1040,737$; HRMS (FAB, NBA + Nal matrix) Calcd. for $\mathrm{C}_{74} \mathrm{H}_{120} \mathrm{~N}_{4} \mathrm{O}_{8} \mathrm{Na}_{12} 1215.9004$ $\left([\mathrm{M}+\mathrm{Na}]^{+}\right)$, Found: 1215.8998 .<smiles>CCOc1ccc(CON(C)C(=O)[C@H](C)NC(=O)C(N)CC(N)=O)c(OCC)c1</smiles>

Asn-Ala-(Me)N-O-TAGb (S26)

Following the general procedure described for Fmoc deprotection, Fmoc-Asn-Ala-(Me)N-O-TAGb (S25) (1.70 g, $1.4 \mathrm{mmol})$ was converted to Asn-Ala-(Me)N-O-TAGb (S26) $(1.38 \mathrm{~g} \text {, quant.) as a white powder. [a }]_{\mathrm{D}}^{24.5}=17.5\left(\mathrm{c} 0.1, \mathrm{CHCl}_{3}\right) ; \mathrm{mp} \mathrm{ca}^{\circ} 190{ }^{\circ} \mathrm{C}$ (decomp.); ${ }^{1} \mathrm{H}$ NMR $\left(500 \mathrm{MHz}, \mathrm{CDCl}_{3}\right) \delta 7.82(1 \mathrm{H}, \mathrm{br} \mathrm{d}, J=8.0 \mathrm{~Hz}), 7.24(1 \mathrm{H}, \mathrm{d}, J=8.0 \mathrm{~Hz}), 6.44-6.43(2 \mathrm{H}, \mathrm{m}$, overlapped), 6.18 $(1 \mathrm{H}$, br s), $5.35(1 \mathrm{H}, \mathrm{br} \mathrm{s}), 5.03-4.97(2 \mathrm{H}, \mathrm{br} \mathrm{m}$, overlapped), $4.83(1 \mathrm{H}, \mathrm{d}, \mathrm{J}=9.5 \mathrm{~Hz}), 4.04-3.93(4 \mathrm{H}, \mathrm{m}$, overlapped), $3.69(1 \mathrm{H}, \mathrm{br} \mathrm{m})$, $3.24(3 \mathrm{H}, \mathrm{s}), 2.68(1 \mathrm{H}, \mathrm{m}), 2.57(1 \mathrm{H}, \mathrm{m}), 1.83-1.74(4 \mathrm{H}, \mathrm{m}$, overlapped), 1.48-1.41 $(4 \mathrm{H}, \mathrm{br} \mathrm{m}$, overlapped), $1.35-1.22(75 \mathrm{H}, \mathrm{br} \mathrm{m}$, overlapped), $0.88(6 \mathrm{H}, \mathrm{t}, J=7.5 \mathrm{~Hz}) ;{ }^{13} \mathrm{C} \mathrm{NMR}\left(125 \mathrm{MHz}, \mathrm{CDCl}_{3} / \mathrm{CD}_{3} \mathrm{OD}=10 / 1\right.$, Signals were complex due to the rotamers.) $\delta 174.1$, 173.5 (two signals), 161.6, 160.5, 159.0, 158.5, 132.9, 131.2, 118.0, 114.6, 104.9, 104.7, 99.9, 99.8, 71.6, 70.2, 68.23, 68.18, 52.1, 45.7, 39.89, 39.87, 38.9, 33.4, 32.0, 29.8-29.2 (many signals overlapped), 26.15, 26.10, 22.7, 17.6, 14.2; IR (KBr) $v\left(\mathrm{~cm}^{-1}\right) 3360$, 2916, 2849, 1660, 1468, 1177, 1130, 719; HRMS (FAB, NBA + Nal matrix) Calcd. for $\mathrm{C}_{59} \mathrm{H}_{110} \mathrm{~N}_{4} \mathrm{O}_{6} \mathrm{Na}: 993.8323\left([\mathrm{M}+\mathrm{Na}]^{+}\right)$, Found: 993.8315. 


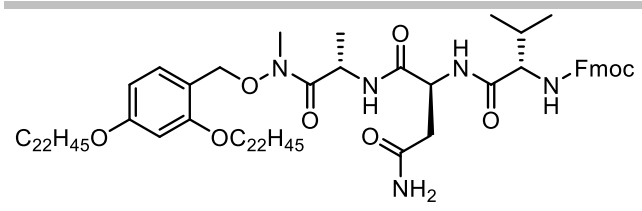

Fmoc-Val-Asn-Ala-(Me)N-O-TAGb (S27)

Following the general procedure described for condensation, Asn-Ala-(Me)N-O-TAGb (S26) (1.34 g, 1.4 mmol) was converted to Fmoc-Val-Asn-Ala-(Me)N-O-TAGb (S27) $(1.66 \mathrm{~g}, 93 \%)$ as a white powder. $[\alpha]_{\mathrm{D}}^{24.5}=9.6\left(\mathrm{c} 0.1, \mathrm{CHCl}_{3}\right) ; \mathrm{mp} 185-190{ }^{\circ} \mathrm{C} ;{ }^{1} \mathrm{H} \mathrm{NMR}(500$ $\mathrm{MHz}, \mathrm{CDCl}_{3}$, Signals were broad and complex due to the rotamers.) $\delta 7.76-7.72(3 \mathrm{H}, \mathrm{br} \mathrm{m}$, overlapped), 7.60-7.57 $(3 \mathrm{H}, \mathrm{br} \mathrm{m}$, overlapped), 7.38-7.35 (2H, br m), $7.28(2 \mathrm{H}, \mathrm{t}, J=7.5 \mathrm{~Hz}), 7.20(1 \mathrm{H}, \mathrm{d}, J=8.5 \mathrm{~Hz}), 6.43-6.40(2 \mathrm{H}$, overlapped), $6.30(1 \mathrm{H}$, br s), 5.86 $(1 \mathrm{H}, \mathrm{br} \mathrm{s}), 5.76(1 \mathrm{H}, \mathrm{br} \mathrm{d}, J=8.5 \mathrm{~Hz}), 5.00-4.92(2 \mathrm{H}, \mathrm{br} \mathrm{m}$, overlapped), 4.86-4.77 $(2 \mathrm{H}$, br m, overlapped), 4.41-4.29 $(2 \mathrm{H}$, br m, overlapped), 4.21-4.14 (2H, br m, overlapped), 4.00-3.91 (4H, br m, overlapped), $3.20(3 \mathrm{H}, \mathrm{br} \mathrm{s}), 2.83(1 \mathrm{H}, \mathrm{br} \mathrm{m}), 2.63(1 \mathrm{H}, \mathrm{br} \mathrm{m})$, $2.16(1 \mathrm{H}, \mathrm{br} \mathrm{m}), 1.81-1.73(4 \mathrm{H}, \mathrm{br} \mathrm{m}$, overlapped $), 1.47-1.40(4 \mathrm{H}, \mathrm{br} \mathrm{m}$, overlapped), 1.35-1.22 (75H, br m, overlapped), $0.99(3 \mathrm{H}, \mathrm{d}$, $J=6.5 \mathrm{~Hz}), 0.95(3 \mathrm{H}, \mathrm{d}, J=7.0 \mathrm{~Hz}), 0.88(6 \mathrm{H}, \mathrm{t}, J=7.5 \mathrm{~Hz}) ;{ }^{13} \mathrm{C} \mathrm{NMR}\left(125 \mathrm{MHz}, \mathrm{CDCl}_{3}\right.$, Signals were broad and complex due to the rotamers.) $\delta 173.5,173.3,171.6,170.1,161.6,159.0,156.7,144.1,143.9,141.4,132.9,127.8,127.22,127.18,125.32,125.29$, $120.1,114.7,104.8,99.8,71.6,68.3,67.2,60.3,49.8,47.3,46.4,37.2,33.5,32.1,31.5,29.8-29.2$ (many signals overlapped), 26.18, 26.16, 22.8, 19.4, 17.8, 17.7, 14.3; IR (KBr) $v\left(\mathrm{~cm}^{-1}\right)$ 3288, 2917, 2849, 1642, 1535, 1468, 1292, 1248, 1180, 1131, 1032, 740, 718; HRMS (FAB, NBA + Nal matrix) Calcd. for $\mathrm{C}_{79} \mathrm{H}_{129} \mathrm{~N}_{5} \mathrm{O}_{9} \mathrm{Na}: 1314.9688\left([\mathrm{M}+\mathrm{Na}]^{+}\right)$, Found: 1314.9697<smiles>CCOc1ccc(CON(C)C(=O)[C@H](C)NC(=O)C(CC(N)=O)NC(=O)C(N)C(C)C)c(OCC)c1</smiles>

Val-Asn-Ala-(Me)N-O-TAGb (S28)

Following the general procedure described for Fmoc deprotection, Fmoc-Val-Asn-Ala-(Me)N-O-TAGb (S27) (1.61 g, $1.2 \mathrm{mmol})$ was converted to Val-Asn-Ala-(Me)N-O-TAGb (S28) (1.33 g, quant.) as a white powder. [a $]_{\mathrm{D}}^{24.5}=7.7\left(\mathrm{c} 0.1, \mathrm{CHCl}_{3}\right) ; \mathrm{mp}^{101-102}{ }^{\circ} \mathrm{C}$; ${ }^{1} \mathrm{H}$ NMR $\left(500 \mathrm{MHz}, \mathrm{CDCl}_{3}\right) \delta 8.39(1 \mathrm{H}, \mathrm{br} \mathrm{d}, J=7.5 \mathrm{~Hz}), 7.61(1 \mathrm{H}, \mathrm{br} \mathrm{d}, J=7.5 \mathrm{~Hz}), 7.22(1 \mathrm{H}, \mathrm{d}, J=9.0 \mathrm{~Hz}), 6.50(1 \mathrm{H}$, br s$), 6.44-$ $6.42(2 \mathrm{H}, \mathrm{m}$, overlapped), $5.78(1 \mathrm{H}, \mathrm{br} \mathrm{s}), 4.97-4.91(2 \mathrm{H}, \mathrm{br} \mathrm{m}$, overlapped), 4.84-4.78 (2H, br m, overlapped), 4.01-3.92 $(4 \mathrm{H}, \mathrm{m}$, overlapped), $3.28(1 \mathrm{H}, \mathrm{d}, J=4.0 \mathrm{~Hz}), 3.22(3 \mathrm{H}, \mathrm{s}), 2.84(1 \mathrm{H}, \mathrm{m}), 2.64(1 \mathrm{H}, \mathrm{m}), 2.24(1 \mathrm{H}, \mathrm{m}), 1.82-1.73(4 \mathrm{H}, \mathrm{m}$, overlapped), $1.47-1.41$ (4H, br m, overlapped), 1.35-1.22 (75H, br m, overlapped), $0.98(3 \mathrm{H}, \mathrm{d}, J=7.0 \mathrm{~Hz}), 0.89-0.83\left(9 \mathrm{H}, \mathrm{m}\right.$, overlapped); ${ }^{13} \mathrm{C}$ NMR $(125$ $\left.\mathrm{MHz}, \mathrm{CDCl}_{3}\right) \delta 174.9,173.4,173.3,170.4,161.6,159.0,132.9,114.7,104.8,99.8,71.6,68.2,60.3,49.6,46.4,37.9,33.5,32.0$, 31.2, 29.8-29.2 (many signals overlapped), 26.1, 22.8, 19.7, 17.7, 16.4, 14.2; IR (KBr) v $\left(\mathrm{cm}^{-1}\right) 3286,2916,2849,1654,1469,1179$, 1131, 719; HRMS (FAB, NBA matrix) Calcd. for $\mathrm{C}_{64} \mathrm{H}_{120} \mathrm{~N}_{5} \mathrm{O}_{7}: 1070.9188\left([\mathrm{M}+\mathrm{Na}]^{+}\right)$, Found: 1070.9191.<smiles>CCCN1C[C@@H](C)CC1C(=O)N[C@@H](C(=O)NC(CC(N)=O)C(=O)N[C@@H](C)C(=O)N(C)OCc1ccc(OCC)cc1OCC)C(C)C</smiles>

Fmoc-4-MePro-Val-Asn-Ala-(Me)N-O-TAGb (S29)

Following the general procedure described for condensation, Val-Asn-Ala-(Me)N-O-TAGb (S28) (1.28 g, 1.2 mmol) was converted to Fmoc-4-MePro-Val-Asn-Ala-(Me)N-O-TAGb (S29) (1.68 g, quant.) as a white powder. [a] $]_{D}^{24.6}=-9.7$ (c $\left.0.1, \mathrm{CHCl}_{3}\right)$; mp 160$161^{\circ} \mathrm{C} ;{ }^{1} \mathrm{H} \mathrm{NMR}\left(500 \mathrm{MHz}, \mathrm{CDCl}_{3}\right.$, Signals were broad and complex due to the rotamers. Those derived from minor rotamers were not described here.) $\delta 7.76(2 \mathrm{H}, \mathrm{br} d, J=6.5 \mathrm{~Hz}), 7.63(1 \mathrm{H}, \mathrm{br} \mathrm{d}, J=7.0 \mathrm{~Hz}), 7.59(2 \mathrm{H}, \mathrm{br} \mathrm{d}, J=7.5 \mathrm{~Hz}), 7.49(1 \mathrm{H}, \mathrm{br} d, J=7.0 \mathrm{~Hz})$, $7.40(2 \mathrm{H}$, br t,$J=7.5 \mathrm{~Hz}), 7.32(2 \mathrm{H}, \mathrm{td}, J=7.5,1.0 \mathrm{~Hz}), 7.23(1 \mathrm{H}, \mathrm{br} \mathrm{d}, J=8.0 \mathrm{~Hz}), 6.43-6.42(2 \mathrm{H}, \mathrm{br} \mathrm{m}$, overlapped), $6.34(1 \mathrm{H}$, br s), $5.85(1 \mathrm{H}$, br s), $5.39(1 \mathrm{H}$, br s), 4.97-4.92 $(2 \mathrm{H}$, br m, overlapped), $4.81(2 \mathrm{H}, \mathrm{br} \mathrm{m}$, overlapped), 4.48-4.34 (4H, br m, overlapped), 4.25 $(1 \mathrm{H}$, br t, $J=6.5 \mathrm{~Hz}), 4.01-3.92(4 \mathrm{H}, \mathrm{br} \mathrm{m}$, overlapped), $3.66(1 \mathrm{H}, \mathrm{brt}, J=7.0 \mathrm{~Hz}), 3.21(3 \mathrm{H}, \mathrm{br} \mathrm{s}), 2.96(1 \mathrm{H}, \mathrm{br} \mathrm{t}, J=10.0 \mathrm{~Hz}), 2.78$ $(1 \mathrm{H}$, br m), $2.65(1 \mathrm{H}$, br m), 2.28-2.07 (3H, br m, overlapped), 1.80-1.73 $(4 \mathrm{H}, \mathrm{br} \mathrm{m}), 1.65(1 \mathrm{H}, \mathrm{br} \mathrm{m}), 1.47-1.41$ (4H, br m, overlapped) 1.35-1.22 (75H, br m, overlapped), $1.06(3 \mathrm{H}, \mathrm{br} d, J=6.0 \mathrm{~Hz}), 0.93(3 \mathrm{H}, \mathrm{d}, J=6.5 \mathrm{~Hz}), 0.90-0.86\left(9 \mathrm{H}, \mathrm{m}\right.$, overlapped); ${ }^{13} \mathrm{C} \mathrm{NMR}(125$ $\mathrm{MHz}, \mathrm{CDCl}_{3}$, Signals were broad and complex due to the rotamers.) $\delta 173.4,173.2,172.3,171.2,170.0,161.6,159.0,156.3,144.1$, 143.8, 141.4, 133.0, 127.9, 127.5, 127.3, 125.2, 120.1, 114.8, 104.8, 99.8, 71.6, 68.3, 68.0, 61.3, 58.7, 54.0, 49.9, 47.3, 46.3, 37.3, 36.7, 33.5, 32.7, 32.1, 30.9, 29.8-29.3 (many signals overlapped), 26.2, 22.8, 19.5, 17.9, 17.8, 17.3, 14.3; IR $(\mathrm{KBr}) v\left(\mathrm{~cm}^{-1}\right) 3286$, 2917, 2849, 1642, 1468, 1418, 1178, 1129, 739, 719; HRMS (FAB, NBA + Nal matrix) Calcd. for $\mathrm{C}_{85} \mathrm{H}_{138} \mathrm{~N}_{6} \mathrm{O}_{10} \mathrm{Na}: 1426.0372$ ([M + $\mathrm{Na}]^{+}$), Found: 1426.0363 .<smiles>CCOc1ccc(CON(C)C(=O)[C@H](C)NC(=O)C(CC(N)=O)NC(=O)[C@H](NC(=O)C2C[C@@H](C)CN2)C(C)C)c(OCC)c1</smiles>

4-MePro-Val-Asn-Ala-(Me)N-O-TAGb (S30)

Following the general procedure described for Fmoc deprotection, Fmoc-4-MePro-Val-Asn-Ala-(Me)N-O-TAGb (S29) (1.64 g, 1.2 mmol) was converted to 4-MePro-Val-Asn-Ala-(Me)N-O-TAGb (S30) (1.38 g, quant.) as a white powder. $[\mathrm{a}]_{\mathrm{D}}{ }^{24.6}=-2.3\left(\mathrm{c} 0.1, \mathrm{CHCl}_{3}\right)$; mp 93-95 ${ }^{\circ} \mathrm{C} ;{ }^{1} \mathrm{H}$ NMR $\left(500 \mathrm{MHz}, \mathrm{CDCl}_{3}\right) \delta 8.31(1 \mathrm{H}, \mathrm{br} \mathrm{d}, J=8.5 \mathrm{~Hz}), 7.78(1 \mathrm{H}, \mathrm{br} \mathrm{d}, J=7.5 \mathrm{~Hz}), 7.58(1 \mathrm{H}$, br d, $J=7.0 \mathrm{~Hz}), 7.23$ $(1 \mathrm{H}$, br m), $6.54(1 \mathrm{H}$, br s), 6.43-6.42 $(2 \mathrm{H}$, br m, overlapped), $5.94(1 \mathrm{H}, \mathrm{br} \mathrm{s}), 4.97-4.92(2 \mathrm{H}, \mathrm{br} \mathrm{m}$, overlapped $), 4.83-4.75(2 \mathrm{H}, \mathrm{m}$, overlapped), $4.28(1 \mathrm{H}, \mathrm{dd}, J=9.5,6.5 \mathrm{~Hz}), 4.01-3.92(4 \mathrm{H}, \mathrm{m}$, overlapped), $3.82(1 \mathrm{H}, \mathrm{m}), 3.21(3 \mathrm{H}, \mathrm{s}), 3.08(1 \mathrm{H}, \mathrm{dd}, J=10.0,6.5 \mathrm{~Hz})$, $2.80(1 \mathrm{H}, \mathrm{m}), 2.65-2.56(2 \mathrm{H}, \mathrm{m}$, overlapped), $2.21(1 \mathrm{H}, \mathrm{m}), 2.13-2.04(2 \mathrm{H}, \mathrm{m}$, overlapped), 1.82-1.68 (5H, m, overlapped), 1.46-1.41 (4H, br m, overlapped), 1.35-1.22 (75H, br m, overlapped), $0.99(3 \mathrm{H}, \mathrm{d}, J=6.5 \mathrm{~Hz}), 0.96(3 \mathrm{H}, \mathrm{d}, J=6.5 \mathrm{~Hz}), 0.91(3 \mathrm{H}, \mathrm{d}, J=7.0 \mathrm{~Hz})$, 
$0.87(6 \mathrm{H}, \mathrm{t}, J=7.0 \mathrm{~Hz}) ;{ }^{13} \mathrm{C}$ NMR $\left(125 \mathrm{MHz}, \mathrm{CDCl}_{3}\right) \delta 176.2,173.6,173.3,171.7,170.2,161.6,159.0,133.0,114.8,104.9,99.8$, $71.6,68.3,60.4,58.1,54.7,50.0,46.3,39.0,37.2,33.5,33.3,32.1,30.9,29.8-29.3$ (many signals overlapped), 26.19, 26.17, 22.8, 19.7, 17.9, 17.7, 17.6, 14.3; IR (KBr) $v\left(\mathrm{~cm}^{-1}\right) 3271,2916,2849,1643,1508,1468,1179,1131,719$; HRMS (FAB, NBA matrix) Calcd. for $\mathrm{C}_{70} \mathrm{H}_{128} \mathrm{~N}_{6} \mathrm{O}_{8}: 1181.9872\left([\mathrm{M}+\mathrm{H}]^{+}\right)$, Found: 1181.9851 .<smiles>CCCNC(=O)N1C[C@H](C)CC1C(=O)N[C@@H](C(=O)NC(CC(N)=O)C(=O)N[C@@H](C)C(=O)N(C)OCc1ccc(OCC)cc1OCC)C(C)C</smiles>

Fmoc-Thr-4-MePro-Val-Asn-Ala-(Me)N-O-TAGb (S31)

Following the general procedure described for condensation, 4-MePro-Val-Asn-Ala-(Me)N-O-TAGb (S30) (657 mg, $0.56 \mathrm{mmol})$ was converted to Fmoc-Thr-4-MePro-Val-Asn-Ala-(Me)N-O-TAGb (S31) $(818 \mathrm{mg}, 98 \%)$ as a white powder. [a] $]_{D}^{24.6}=-11.9(c 0.1$, $\left.\mathrm{CHCl}_{3}\right) ; \mathrm{mp} 115-125^{\circ} \mathrm{C} ;{ }^{1} \mathrm{H}$ NMR $\left(500 \mathrm{MHz}, \mathrm{CDCl}_{3}\right.$, Signals were broad and complex due to the rotamers. Those derived from minor rotamers were not described here.) $\delta 7.78(1 \mathrm{H}, \mathrm{br} d, J=8.0 \mathrm{~Hz}), 7.74(2 \mathrm{H}, \mathrm{br} \mathrm{d}, J=7.5 \mathrm{~Hz}), 7.59(2 \mathrm{H}, \mathrm{br} d, J=7.5 \mathrm{~Hz}), 7.38(2 \mathrm{H}, \mathrm{br}$ $\mathrm{t}, J=7.5 \mathrm{~Hz}), 7.30(2 \mathrm{H}, \mathrm{brtd}, J=7.5,2.0 \mathrm{~Hz}), 7.30(1 \mathrm{H}, \mathrm{br} \mathrm{d}, J=8.5 \mathrm{~Hz}), 7.15(1 \mathrm{H}, \mathrm{br} \mathrm{d}, J=9.0 \mathrm{~Hz}), 6.95(1 \mathrm{H}, \mathrm{br} \mathrm{s}), 6.44-6.40(2 \mathrm{H}$, br m, overlapped), $6.36(1 \mathrm{H}, \mathrm{br} \mathrm{s}), 6.23(1 \mathrm{H}, \mathrm{br} \mathrm{d}, J=8.0 \mathrm{~Hz}), 5.05-4.96(3 \mathrm{H}, \mathrm{br} \mathrm{m}$, overlapped), $4.80(1 \mathrm{H}, \mathrm{br} \mathrm{d}, \mathrm{J}=10.0 \mathrm{~Hz}), 4.71$ $(1 \mathrm{H}, \mathrm{br} d d, J=9.5,4.0 \mathrm{~Hz}), 4.66-4.49(3 \mathrm{H}, \mathrm{br} \mathrm{m}$, overlapped), $4.45(1 \mathrm{H}, \mathrm{dd}, J=11.0,7.5 \mathrm{~Hz}), 4.35(1 \mathrm{H}, \mathrm{dd}, J=10.5,7.0 \mathrm{~Hz}), 4.30$ $(1 \mathrm{H}, \mathrm{br} \mathrm{m}), 4.20(1 \mathrm{H}, \mathrm{t}, J=7.0 \mathrm{~Hz}), 4.05(1 \mathrm{H}, \mathrm{brt}, J=8.0 \mathrm{~Hz}), 4.01-3.90(4 \mathrm{H}, \mathrm{br} \mathrm{m}$, overlapped), $3.31(1 \mathrm{H}, \mathrm{br} \mathrm{t}, J=9.5 \mathrm{~Hz}), 3.21(3 \mathrm{H}$, s), $2.79(1 \mathrm{H}, \mathrm{br} \mathrm{m}), 2.70(1 \mathrm{H}, \mathrm{br} \mathrm{m}), 2.47(1 \mathrm{H}, \mathrm{br} \mathrm{m}), 2.25-2.17(2 \mathrm{H}, \mathrm{br} \mathrm{m}$, overlapped), $1.93(1 \mathrm{H}, \mathrm{br} \mathrm{m}), 1.82-1.73(4 \mathrm{H}, \mathrm{br} \mathrm{m}$, overlapped), 1.47-1.41 (4H, br m, overlapped), 1.35-1.22 (78H, br m, overlapped), $1.09(3 \mathrm{H}, \mathrm{d}, J=6.5 \mathrm{~Hz}), 0.95(3 \mathrm{H}, \mathrm{d}, J=7.0 \mathrm{~Hz})$, $0.88(9 \mathrm{H}, \mathrm{t}, J=7.0 \mathrm{~Hz}) ;{ }^{13} \mathrm{C} \mathrm{NMR}\left(125 \mathrm{MHz}, \mathrm{CDCl}_{3}\right.$, Signals were broad and complex due to the rotamers.) $\delta 173.8,173.1,172.2$, $171.6,171.2,169.8,161.6,159.0,156.4,143.9,143.8,141.4,133.0,127.9,127.2,125.23,125.19,120.10,120.08,114.8,104.8$, $99.8,71.6,68.3,68.1,67.1,61.6,58.0,56.8,54.6,50.5,47.3,46.1,37.8,37.2,33.5,32.9,32.1,31.2,29.8-29.3$ (many signals overlapped), 26.2, 22.8, 19.6, 18.6, 18.1, 18.0, 17.4, 14.3; IR (KBr) v( $\left.\mathrm{cm}^{-1}\right)$ 3302, 2917, 2849, 1644, 1508, 1467, 1264, 1179, 740, 721; HRMS (FAB, NBA + Nal matrix) Calcd. for $\mathrm{C}_{89} \mathrm{H}_{145} \mathrm{~N}_{7} \mathrm{O}_{12} \mathrm{Na:} 1527.0849\left([\mathrm{M}+\mathrm{Na}]^{+}\right)$, Found: 1527.0854.<smiles>CCOc1ccc(CON(C)C(=O)[C@H](C)NC(=O)C(CC(N)=O)NC(=O)[C@H](NC(=O)C2C[C@@H](C)CN2C(=O)[C@@H](N)[C@H](C)O)C(C)C)c(OCC)c1</smiles>

\section{Thr-4-MePro-Val-Asn-Ala-(Me)N-O-TAGb (S32)}

Following the general procedure described for Fmoc deprotection, Fmoc-Thr-4-MePro-Val-Asn-Ala-(Me)N-O-TAGb (S31) (768 $\mathrm{mg}, 0.51 \mathrm{mmol}$ ) was converted to Thr-4-MePro-Val-Asn-Ala-(Me)N-O-TAGb (S32) $\left(664 \mathrm{mg}\right.$, quant.) as a white powder. [a] ${ }_{\mathrm{D}}^{24.6}=-9.7$ (c $\left.0.1, \mathrm{CHCl}_{3}\right) ; \mathrm{mp} 70-78{ }^{\circ} \mathrm{C} ;{ }^{1} \mathrm{H} \mathrm{NMR}\left(500 \mathrm{MHz}, \mathrm{CDCl}_{3}\right.$, Signals were broad and complex due to the rotamers. Those derived from minor rotamers were not described here.) $\delta 7.97(1 \mathrm{H}, \mathrm{br} \mathrm{d}, J=8.0 \mathrm{~Hz}), 7.35(1 \mathrm{H}, \mathrm{br} \mathrm{d}, J=9.5 \mathrm{~Hz}), 7.25(1 \mathrm{H}, \mathrm{br} \mathrm{m}), 7.16(1 \mathrm{H}, \mathrm{br} \mathrm{s})$, $6.96(1 \mathrm{H}$, br s), 6.43-6.41 (2H, br m, overlapped), 5.04-4.91 (3H, br m, overlapped), $4.80(1 \mathrm{H}, \mathrm{br} \mathrm{m}), 4.69-4.60(2 \mathrm{H}$, br m, overlapped), 4.15-4.09 (2H, br m, overlapped), 4.01-3.92 $(4 \mathrm{H}$, br m, overlapped), $3.84(1 \mathrm{H}, \mathrm{br} \mathrm{d}, J=5.0 \mathrm{~Hz}), 3.21(3 \mathrm{H}, \mathrm{s}), 3.15(1 \mathrm{H}$, br t, $J=9.5 \mathrm{~Hz}), 2.75(1 \mathrm{H}, \mathrm{br} \mathrm{m}), 2.65(1 \mathrm{H}, \mathrm{br} \mathrm{m}), 2.47(1 \mathrm{H}, \mathrm{br} \mathrm{m}), 2.24-2.08(3 \mathrm{H}, \mathrm{br} \mathrm{m}$, overlapped), 1.87-1.73 $(5 \mathrm{H}, \mathrm{br} \mathrm{m}$, overlapped), 1.47-1.40 (4H, br m, overlapped), 1.35-1.22 (78H, br m, overlapped), $1.09(3 \mathrm{H}, \mathrm{d}, J=6.5 \mathrm{~Hz}), 0.95(3 \mathrm{H}, \mathrm{d}, J=6.0 \mathrm{~Hz})$, $0.87(9 \mathrm{H}, \mathrm{t}, J=7.5 \mathrm{~Hz}) ;{ }^{13} \mathrm{C}$ NMR $\left(125 \mathrm{MHz}, \mathrm{CDCl}_{3}\right) \delta 174.0,173.3,173.2,172.4,171.5,169.9,161.6,159.0,133.0,114.8,104.9$, $99.8,71.6,70.2,68.3,61.7,57.6,56.3,54.5,50.5,46.0,37.6,37.3,33.5,32.8,32.3,32.1,29.8-29.3$ (many signals overlapped), 26.2, 22.8, 19.5, 18.2, 17.9, 17.4, 14.3; IR (KBr) $v\left(\mathrm{~cm}^{-1}\right) 3297,2917,2849,1645,1508,1468,1178,1131,719$; HRMS (FAB, NBA matrix) Calcd. for $\mathrm{C}_{74} \mathrm{H}_{136} \mathrm{~N}_{7} \mathrm{O}_{10}: 1283.0349\left([\mathrm{M}+\mathrm{H}]^{+}\right)$, Found: 1283.0349 .<smiles>CCOc1ccc(CON(C)C(=O)[C@H](C)NC(=O)C(CC(N)=O)NC(=O)[C@H](NC(=O)[C@H]2C[C@H](C)CN2C(=O)[C@@H](NC(=O)NCC(C)=O)C(C)C)C(C)C)c(OCC)c1</smiles>

Oleic acid-Thr-4-MePro-Val-Asn-Ala-(Me)N-O-TAGb (12)

Following the general procedure described for condensation using oleic acid instead of Fmoc protected amino acid, Thr-4-MeProVal-Asn-Ala-(Me)N-O-TAGb (S32) $(610 \mathrm{mg}, 0.48 \mathrm{mmol})$ was converted to oleic acid-Thr-4-MePro-Val-Asn-Ala-(Me)N-O-TAGb (12) $(707 \mathrm{mg}, 96 \%)$ as a white powder. $[\alpha]_{\mathrm{D}}^{24.6}=-8.5\left(\mathrm{c} 0.1, \mathrm{CHCl}_{3}\right) ; \mathrm{mp} 155-163{ }^{\circ} \mathrm{C} ;{ }^{1} \mathrm{H} \mathrm{NMR}\left(500 \mathrm{MHz}, \mathrm{CDCl}_{3}\right) \delta 7.84(1 \mathrm{H}, \mathrm{br} \mathrm{d}, J=9.0$ $\mathrm{Hz}), 7.28(1 \mathrm{H}$, br d, $J=7.5 \mathrm{~Hz}), 7.24(1 \mathrm{H}, \mathrm{brd}, J=8.5 \mathrm{~Hz}), 7.16(1 \mathrm{H}$, br d, $J=9.0 \mathrm{~Hz}), 7.11(1 \mathrm{H}$, br s), $6.76(1 \mathrm{H}, b r \mathrm{~d}, J=8.0 \mathrm{~Hz})$, $6.58(1 \mathrm{H}$, br s), 6.44-6.42 $(2 \mathrm{H}$, br m, overlapped), 5.36-5.29 $(2 \mathrm{H}, \mathrm{m}$, overlapped $), 5.01-4.92(4 \mathrm{H}$, br m, overlapped $), 4.80(1 \mathrm{H}$, br d, $J$ $=9.5 \mathrm{~Hz}), 4.61-4.58(2 \mathrm{H}$, br m, overlapped $), 4.29(1 \mathrm{H}, \mathrm{br} \mathrm{m}), 4.06(1 \mathrm{H}, \mathrm{br} \mathrm{m}), 4.02-3.92(4 \mathrm{H}, \mathrm{br} \mathrm{m}$, overlapped $), 3.35(1 \mathrm{H}, \mathrm{br} \mathrm{t}, J=9.5$ $\mathrm{Hz}), 3.21(3 \mathrm{H}, \mathrm{s}), 2.81-2.76(1 \mathrm{H}, \mathrm{br} \mathrm{m}), 2.70-2.66(1 \mathrm{H}, \mathrm{br} \mathrm{m}), 2.46(1 \mathrm{H}, \mathrm{br} \mathrm{m}), 2.25-2.15(6 \mathrm{H}, \mathrm{br} \mathrm{m}$, overlapped $), 1.99(3 \mathrm{H}, \mathrm{br} \mathrm{m}), 1.89$ $(1 \mathrm{H}, \mathrm{br} \mathrm{m}), 1.82-1.73(4 \mathrm{H}, \mathrm{br} \mathrm{m}), 1.62(2 \mathrm{H}, \mathrm{br} \mathrm{m}), 1.44(4 \mathrm{H}, \mathrm{br} \mathrm{m}), 1.35-1.22(98 \mathrm{H}, \mathrm{br} \mathrm{m}$, overlapped), $1.09(3 \mathrm{H}, \mathrm{d}, \mathrm{J}=6.5 \mathrm{~Hz}), 0.94$ $(3 \mathrm{H}, \mathrm{d}, J=6.5 \mathrm{~Hz}), 0.88-0.86\left(12 \mathrm{H}, \mathrm{m}\right.$, overlapped); ${ }^{13} \mathrm{C} \mathrm{NMR}\left(125 \mathrm{MHz}, \mathrm{CDCl}_{3}\right) \delta 173.7,173.4,173.2,172.2,171.6,171.3,169.8$, 161.7, 159.0, 133.0, 130.1, 129.9, 114.7, 104.9, 99.8, 71.7, 68.3, 67.9, 61.6, 57.8, 54.6, 54.5, 50.3, 46.1, 37.8, 37.2, 36.6, 33.6, 32.9, 32.1, 31.6, 29.9-29.3 (many signals overlapped), 27.3, 26.2, 25.7, 22.8, 19.5, 18.8, 18.0, 17.9, 17.3, 14.3; IR (KBr) $v\left(\mathrm{~cm}^{-1}\right) 3284$, 


\section{Selective deprotection of TAG benzyl group under acid conditions and reduction to the aldehydes} Scheme 3c.

(n)

$11(55.1 \mathrm{mg}, 0.032 \mathrm{mmol}, 1.0 \mathrm{eq})$ and $12(50.0 \mathrm{mg}, 0.032 \mathrm{mmol}, 1.0 \mathrm{eq})$ was dissolved into $30 \% \mathrm{TFA} / \mathrm{CHCl}_{3}(3.2 \mathrm{~mL}, 0.01 \mathrm{M}$ for each substrate) at room temperature, and the solution was stirred for $10 \mathrm{~min}$. The reaction mixture was subsequently cooled to $0{ }^{\circ} \mathrm{C}$ and $\mathrm{MeOH}\left(19 \mathrm{~mL}, 6\right.$-fold excess of TFA/ $\mathrm{CHCl}_{3}$ ) was added. The resulting heterogeneous solution was stirred for a further $30 \mathrm{~min}$ at $0{ }^{\circ} \mathrm{C}$. The precipitate was filtered and washed with additional $\mathrm{MeOH}$ to afford the crude cake 11. On the other hand, the filtrate was concentrated in vacuo to afford the crude 10 . The crude products were purified by PLC on silica gel $\left(\mathrm{CHCl}_{3} / \mathrm{MeOH}=5 / 1\right.$ as eluent) to give 11 (53.5 mg, 97\%) as a white powder and hydroxamic acid 10 (22.2 mg, 85\%) as a white powder respectively.

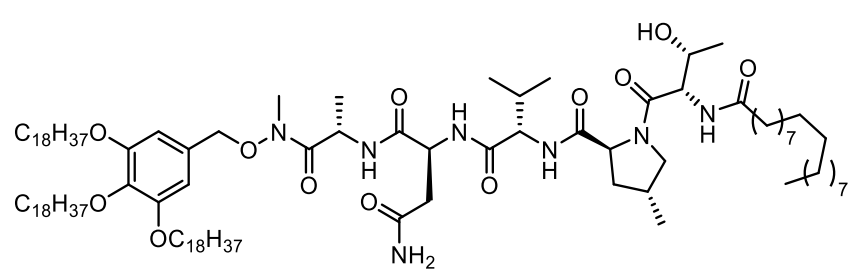

Stearic acid-Thr-4-MePro-Val-Asn-Ala-(Me)N-O-TAGa (11)

Following the general procedure described for condensation using stearic acid instead of Fmoc protected amino acid, Thr-4MePro-Val-Asn-Ala-(Me)N-O-TAGa (8) $(0.300 \mathrm{~g}, 0.21 \mathrm{mmol})$ was converted to stearic acid-Thr-4-MePro-Val-Asn-Ala-(Me)N-O-TAGa (11) $(0.356 \mathrm{~g} \text {, quant.) as a white powder. [a }]_{\mathrm{D}}^{24.6}=-15.5\left(\mathrm{c} 0.1, \mathrm{CHCl}_{3}\right) ; \mathrm{mp} 153-154{ }^{\circ} \mathrm{C} ;{ }^{1} \mathrm{H} \mathrm{NMR}(500 \mathrm{MHz}, \mathrm{CDCl} 3) \delta 7.80(1 \mathrm{H}, \mathrm{br} \mathrm{d}$, $J=8.5 \mathrm{~Hz}), 7.33(1 \mathrm{H}$, br d, $J=7.5 \mathrm{~Hz}), 7.11-7.08(2 \mathrm{H}$, br m, overlapped), $6.81(1 \mathrm{H}, \mathrm{br} \mathrm{d}, J=8.5 \mathrm{~Hz}), 6.57(2 \mathrm{H}, \mathrm{s}), 6.39(1 \mathrm{H}, \mathrm{br} \mathrm{s})$, $4.98(1 \mathrm{H}, \mathrm{dd}, J=8.5,4.0 \mathrm{~Hz}), 4.96-4.90(2 \mathrm{H}, \mathrm{m}$, overlapped), $4.85(1 \mathrm{H}, \mathrm{br} \mathrm{d}, J=10.0 \mathrm{~Hz}), 4.79(1 \mathrm{H}, \mathrm{br} \mathrm{d}, J=10.5 \mathrm{~Hz}), 4.59(1 \mathrm{H}, \mathrm{dd}$, $J=9.0,4.0 \mathrm{~Hz}), 4.55(1 \mathrm{H}, \mathrm{dd}, J=9.0,6.5 \mathrm{~Hz}), 4.49(1 \mathrm{H}, \mathrm{br} \mathrm{s}), 4.29(1 \mathrm{H}, \mathrm{br} \mathrm{m}), 4.03(1 \mathrm{H}, \mathrm{br} \mathrm{dd}, J=9.5,7.5 \mathrm{~Hz}), 3.98-3.92(6 \mathrm{H}, \mathrm{m}$, overlapped), $3.36(1 \mathrm{H}, \mathrm{t}, J=9.5 \mathrm{~Hz}), 3.18(3 \mathrm{H}, \mathrm{s}), 2.80(1 \mathrm{H}, \mathrm{m}), 2.70(1 \mathrm{H}, \mathrm{m}), 2.46(1 \mathrm{H}, \mathrm{m}), 2.25-2.15(4 \mathrm{H}, \mathrm{m}$, overlapped), $1.90(1 \mathrm{H}$, $\mathrm{m}), 1.82-1.70(6 \mathrm{H}, \mathrm{m}$, overlapped), 1.65-1.56 (2H, m), 1.49-1.43 (6H, br m, overlapped), 1.36-1.22 (89H, br m, overlapped), 1.09 (3H, $\mathrm{t}, J=7.0 \mathrm{~Hz}), 0.94(3 \mathrm{H}, \mathrm{t}, J=6.5 \mathrm{~Hz}), 0.89-0.86\left(15 \mathrm{H}, \mathrm{m}\right.$, overlapped); ${ }^{13} \mathrm{C}$ NMR $\left(125 \mathrm{MHz}, \mathrm{CDCl}_{3}\right) \delta 173.8,173.5,173.4,172.2$, 171.7, 171.5, 170.0, 153.5, 138.8, 129.4, 107.7, 73.6, 69.3, 67.8, 61.5, 58.0, 54.7, 54.6, 50.6, 46.2, 37.8, 37.1, 36.7, 34.1, 33.0, 32.1, 31.3, 30.5, 29.9-29.5 (many signals overlapped), 26.3, 25.8, 22.8, 19.5, 18.9, 17.9, 17.8, 17.3, 14.3; $\mathrm{IR}(\mathrm{KBr}) v\left(\mathrm{~cm}^{-1}\right) 3281,2916$ 2849, 1638, 1543, 1468, 1439, 1239, 1121, 719; HRMS (FAB, NBA + Nal matrix) Calcd. for $\mathrm{C}_{102} \mathrm{H}_{189} \mathrm{~N}_{7} \mathrm{O}_{12} \mathrm{Na}: 1727.4292\left([\mathrm{M}+\mathrm{Na}]^{+}\right)$, Found: 1727.4307.

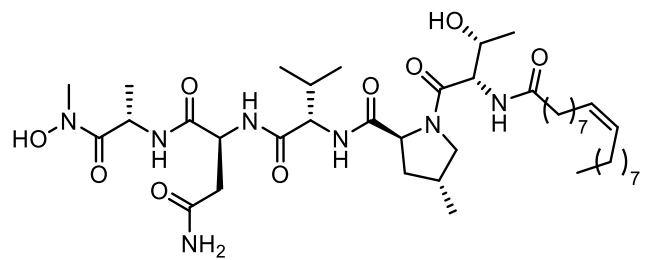

Oleic acid-Thr-4-MePro-Val-Asn-Ala-(Me)N-OH (10)

12 (350 mg, $0.23 \mathrm{mmol}$ ) was dissolved into $30 \%$ trifluoroacetic acid (TFA)/ $\mathrm{CHCl}_{3}(11 \mathrm{~mL}, 0.02 \mathrm{M}$ for substrate) at room temperature, and the solution was stirred for $20 \mathrm{~min}$. The reaction mixture was subsequently cooled to $0{ }^{\circ} \mathrm{C}$ and $\mathrm{MeOH}(66 \mathrm{~mL}, 6$-fold excess of TFA $/ \mathrm{CHCl}_{3}$ ) was added, and the resulting heterogeneous solution was stirred for a further 30 min at $0{ }^{\circ} \mathrm{C}$. The precipitate was filtered and washed with additional $\mathrm{MeOH}$, and the filtrate was concentrated in vacuo. The residue was purified by flash column chromatography on silica gel $\left(\mathrm{CHCl}_{3} / \mathrm{MeOH}=50 / 1\right.$ to $10 / 1$ as eluent) to afford hydroxamic acid $10(160 \mathrm{mg}, 88 \%)$ as a white powder. $[\alpha]_{D}^{24.6}=-32.1$ (c 0.1, DMSO); mp 193-196 ${ }^{\circ} \mathrm{C} ;{ }^{1} \mathrm{H}$ NMR $(500 \mathrm{MHz}$, DMSO-d $) \delta 10.05(1 \mathrm{H}, \mathrm{s}), 8.15(1 \mathrm{H}, \mathrm{d}, J=8.0 \mathrm{~Hz}), 7.86(1 \mathrm{H}, \mathrm{d}, J$ $=7.5 \mathrm{~Hz}), 7.82(1 \mathrm{H}, \mathrm{d}, J=8.5 \mathrm{~Hz}), 7.59(1 \mathrm{H}, \mathrm{br} \mathrm{d}, J=7.5 \mathrm{~Hz}), 7.33(1 \mathrm{H}, \mathrm{br} \mathrm{s}), 6.89(1 \mathrm{H}, \mathrm{br} \mathrm{s}), 5.35-5.29(2 \mathrm{H}, \mathrm{m}$, overlapped), 4.76 $(1 \mathrm{H}, \mathrm{br} \mathrm{m}), 4.65(1 \mathrm{H}, \mathrm{d}, J=6.0 \mathrm{~Hz}), 4.55-4.48(2 \mathrm{H}, \mathrm{m}$, overlapped), $4.42(1 \mathrm{H}, \mathrm{t}, J=7.5 \mathrm{~Hz}), 4.12(1 \mathrm{H}, \mathrm{dd}, J=8.0,6.0 \mathrm{~Hz}), 3.83-3.76$ (2H, m, overlapped), $3.25(1 \mathrm{H}, \mathrm{t}, J=9.0 \mathrm{~Hz}), 3.08(3 \mathrm{H}, \mathrm{s}), 2.56(1 \mathrm{H}, \mathrm{dd}, J=11.0,6.5 \mathrm{~Hz}), 2.39-2.30(2 \mathrm{H}, \mathrm{m}$, overlapped), 2.17-2.06 (2H, m, overlapped), 2.01-1.91 (6H, m, overlapped), $1.64(1 \mathrm{H}, \mathrm{m}), 1.45(2 \mathrm{H}, \mathrm{br} \mathrm{m}), 1.30-1.20(2 \mathrm{HH}$, br m, overlapped), $1.16(3 \mathrm{H}, \mathrm{d}, J$ $=7.0 \mathrm{~Hz}), 1.08(3 \mathrm{H}, \mathrm{d}, J=6.0 \mathrm{~Hz}), 0.97(3 \mathrm{H}, \mathrm{d}, J=6.5 \mathrm{~Hz}), 0.86-0.80\left(9 \mathrm{H}, \mathrm{m}\right.$, overlapped); ${ }^{13} \mathrm{C} \mathrm{NMR}\left(125 \mathrm{MHz}, \mathrm{DMSO}-d_{6}\right) \delta 172.2$, 171.7, 171.5, 171.4, 170.8, 170.1, 169.4, 129.7, 67.0, 59.2, 57.5, 56.1, 54.0, 49.3, 45.0, 36.7, 36.6, 35.9, 34.9, 32.0, 31.3, 30.7, 29.228.6 (many signals overlapped), 26.64, 26.60, 25.3, 22.1, 19.3, 19.2, 17.9, 17.3, 17.2, 14.0; IR (KBr) $v\left(\mathrm{~cm}^{-1}\right) 3283,2924,2854$, 1636, 1540, 1435, 1199; HRMS (ESI+) Calcd. for $\mathrm{C}_{41} \mathrm{H}_{73} \mathrm{~N}_{7} \mathrm{O}_{9} \mathrm{Na}: 830.5367\left([\mathrm{M}+\mathrm{Na}]^{+}\right)$, Found: 830.5358. 


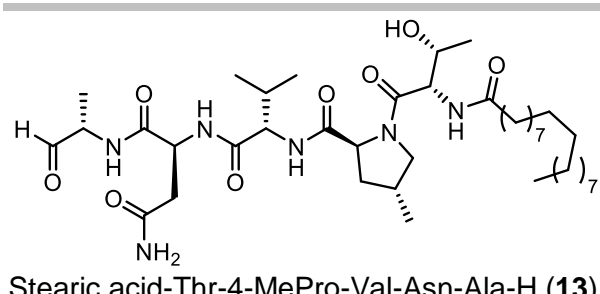

Stearic acid-Thr-4-MePro-Val-Asn-Ala-H (13)

To a solution of $11(50 \mathrm{mg}, 0.029 \mathrm{mmol}, 1.0 \mathrm{eq})$ in dehydrated THF $(2.9 \mathrm{~mL}, 0.01 \mathrm{M})$ was added dropwise $1.0 \mathrm{M} \mathrm{LiAlH}(t-\mathrm{BuO}))_{3}$ in dehydrated THF (293 mL, $10 \mathrm{eq})$ at room temperature. After stirring for $1 \mathrm{~h}$ at room temperature, the reaction mixture was then treated with aqueous $1 \mathrm{~N} \mathrm{HCl}(1.5 \mathrm{~mL})$ at $0{ }^{\circ} \mathrm{C}$ to quench the excess $\mathrm{LiAlH}(t-\mathrm{BuO})_{3}$. After stirring for 10 min at room temperature, $\mathrm{MeOH}(15 \mathrm{~mL})$ was added. The resulting heterogeneous solution was stirred for a further $30 \mathrm{~min}$ at room temperature, and the precipitate was filtered and washed with additional $\mathrm{MeOH}$. The filtrate was roughly concentrated in vacuo, poured into a separatory funnel containing aqueous $1 \mathrm{~N} \mathrm{HCl}(10 \mathrm{~mL})$, and extracted with $\mathrm{CHCl}_{3}(3 \times 10 \mathrm{~mL})$. The combined organic extracts were dried with $\mathrm{Na}_{2} \mathrm{SO}_{4}$, filtered, and concentrated in vacuo. The residue was purified by PLC on silica gel $\left(\mathrm{CHCl}_{3} / \mathrm{MeOH}=5 / 1\right.$ as eluent) to afford aldehyde $13(13.7 \mathrm{mg}, 61 \%)$ as a white powder. $[\alpha]_{\mathrm{D}}^{24.6}=-35.6\left(c 0.1, \mathrm{CHCl}_{3}\right) ; \mathrm{mp} 176-177{ }^{\circ} \mathrm{C} ;{ }^{1} \mathrm{H} \mathrm{NMR}\left(500 \mathrm{MHz}, \mathrm{DMSO}-d_{6}\right) \delta 9.34$ $(1 \mathrm{H}, \mathrm{s}), 8.13(1 \mathrm{H}, \mathrm{d}, J=6.5 \mathrm{~Hz}), 8.10(1 \mathrm{H}, \mathrm{d}, J=8.5 \mathrm{~Hz}), 7.87(2 \mathrm{H}$, app d, $J=8.5 \mathrm{~Hz}$, overlapped), $7.37(1 \mathrm{H}, \mathrm{br} \mathrm{s}), 6.92(1 \mathrm{H}, \mathrm{br} \mathrm{s})$, 4.67 $(1 \mathrm{H}, \mathrm{br} \mathrm{m}), 4.55-4.47(2 \mathrm{H}, \mathrm{m}$, overlapped), $4.41(1 \mathrm{H}, \mathrm{br} \mathrm{m}), 4.03(1 \mathrm{H}, \mathrm{m}), 3.83-3.77(2 \mathrm{H}, \mathrm{m}$, overlapped), $3.27(1 \mathrm{H}, \mathrm{br} \mathrm{m}), 2.52$ $(1 \mathrm{H}, \mathrm{m}$, overlapped with solvent residual signals), $2.34(1 \mathrm{H}, \mathrm{m}), 2.12(2 \mathrm{H}, \mathrm{m}), 2.01-1.92(2 \mathrm{H}$, m, overlapped), $1.65(1 \mathrm{H}, \mathrm{m}), 1.45(2 \mathrm{H}$, br m), 1.29-1.18 (30H, br m, overlapped), $1.14(3 \mathrm{H}, \mathrm{d}, J=7.5 \mathrm{~Hz}), 1.08(3 \mathrm{H}, \mathrm{d}, J=6.0 \mathrm{~Hz}), 0.97(3 \mathrm{H}, \mathrm{d}, J=6.5 \mathrm{~Hz}), 0.86-0.82(9 \mathrm{H}$, $\mathrm{m}$, overlapped); ${ }^{13} \mathrm{C}$ NMR $\left(125 \mathrm{MHz}\right.$, DMSO- $\left.d_{6}\right) \delta 201.3,172.2,171.8,171.4,171.3,170.6,169.4,66.9,59.2,56.2,53.9,49.5,36.7$, $36.5,34.8,32.0,31.3,30.3,29.0-28.7$ (many signals overlapped), 25.2, 22.1, 19.3, 19.1, 18.0, 17.2, 14.0, 13.5; IR $(\mathrm{KBr}) v\left(\mathrm{~cm}^{-1}\right)$ 3286, 2920, 2851, 1639, 1541, 1419, 1239, 1066; HRMS (ESI+) Calcd. for $\mathrm{C}_{40} \mathrm{H}_{72} \mathrm{~N}_{6} \mathrm{O}_{8} \mathrm{Na}: 787.5309\left([\mathrm{M}+\mathrm{Na}]^{+}\right)$, Found: 787.5315 .

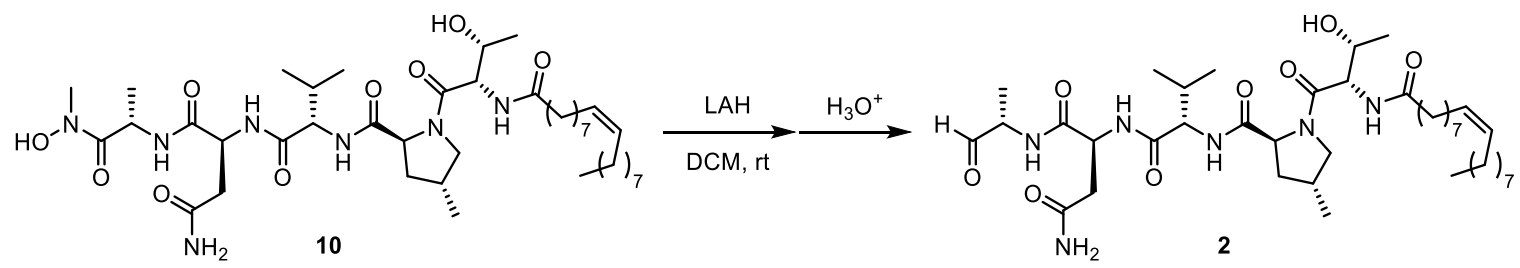

To a solution of $10(20.0 \mathrm{mg}, 0.025 \mathrm{mmol}, 1.0 \mathrm{eq})$ in dehydrated DCM $(2.5 \mathrm{~mL}, 0.01 \mathrm{M})$ was added dropwise $0.2 \mathrm{M} \mathrm{LiAlH}_{4}$ in dehydrated THF (pre-prepared, $0.272 \mathrm{~mL}, 2.2 \mathrm{eq}$ ) at $0{ }^{\circ} \mathrm{C}$. After stirring for $40 \mathrm{~min}$ at room temperature, the reaction mixture was then treated with aqueous $1 \mathrm{~N} \mathrm{HCl}(1.2 \mathrm{~mL})$ at $0{ }^{\circ} \mathrm{C}$ to quench the excess $\mathrm{LiAlH}_{4}$. After stirring for $10 \mathrm{~min}$ at room temperature, $\mathrm{MeOH}(13$ $\mathrm{mL}$ ) was added. The resulting heterogeneous solution was stirred for a further $30 \mathrm{~min}$ at room temperature, and the precipitate was filtered and washed with additional $\mathrm{MeOH}$. The filtrate was roughly concentrated in vacuo, poured into a separatory funnel containing aqueous $1 \mathrm{~N} \mathrm{HCl}(10 \mathrm{~mL})$, and extracted with $\mathrm{CHCl}_{3}(3 \times 10 \mathrm{~mL})$. The combined organic extracts were dried with $\mathrm{Na}_{2} \mathrm{SO}_{4}$, filtered, and concentrated in vacuo. The residue was purified by PLC on silica gel $\left(\mathrm{CHCl}_{3} / \mathrm{MeOH}=5 / 1\right.$ as eluent) to afford aldehyde 2 (8.1 $\mathrm{mg}, 43 \%)$ as a white powder.

\section{References}

[1] a) J. R. D. Valle, M. Goodman, J. Org. Chem. 2003, 68, 3923-3931; b) M. D. Shoulders, J. A. Hodges, R. T. Raines, J. Am. Chem. Soc. 2006, 128, 8112-8113.

[2] Y. Hayashi, W. Fukasawa, T. Hirose, M. Iwatsuki, R. Hokari, A. Ishiyama, M. Kanaida, K. Nonaka, A. Také, K. Otoguro, S. Ōmura, K. Shiomi, T. Sunazuka, Org. Lett. 2019, 21, 2180-2184.

[3] a) P. Marfey, Carisberg Res. Commun. 1984, 49, 591-596; b) K. Fujii, Y. Ikai, T. Mayumi, H. Oka, M. Suzuki, K. Harada, Anal. Chem. 1997, 69, 3346-3352.

[4] a) H. Tamiaki, T. Obata, Y. Azefu, K. Toma, Bull. Chem. Soc. Jpn. 2001, 74, 733-738; b) T. Hirose, T. Kasai, T. Akimoto, A. Endo, A. Sugawara, K. Nagasawa, K. Shiomi, S. Ōmura, T. Sunazuka, Tetrahedron 2011, 67, 6633-6643.

[5] Tana, G.; Kitada, S.; Fujita, S.; Okada, Y.; Kim, S.; Chiba, K. Chem. Commun. 2010, 46, 8219-8221.

[6] Brown, H. C.; McFarlin, R. F. J. Am. Chem. Soc. 1958, 80, 5372-5376. 


\section{NMR spectra of the products}

${ }^{1} \mathrm{H}$ NMR of HO-TAGa (S3) $\left(500 \mathrm{MHz}, \mathrm{CDCl}_{3}\right)$

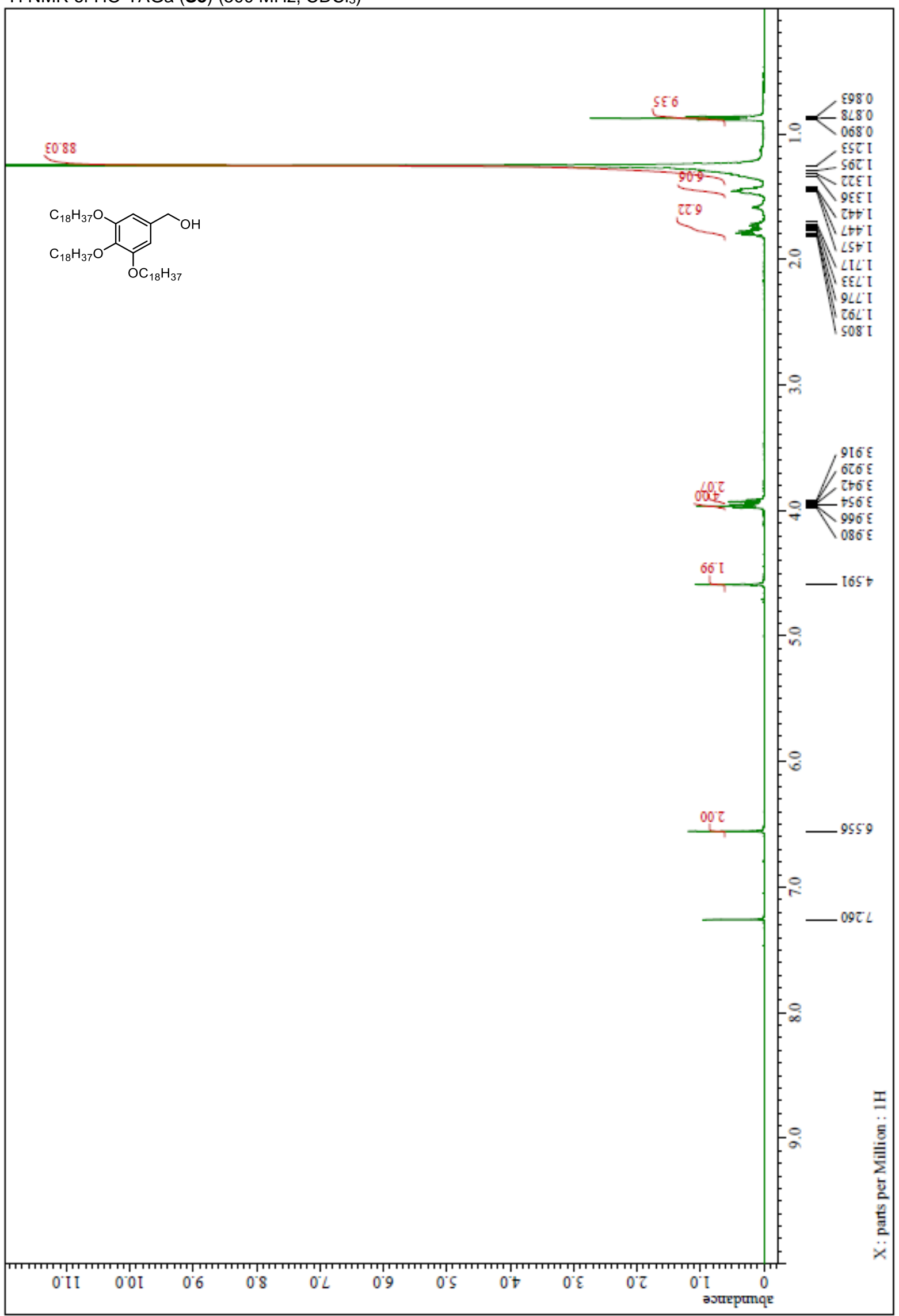




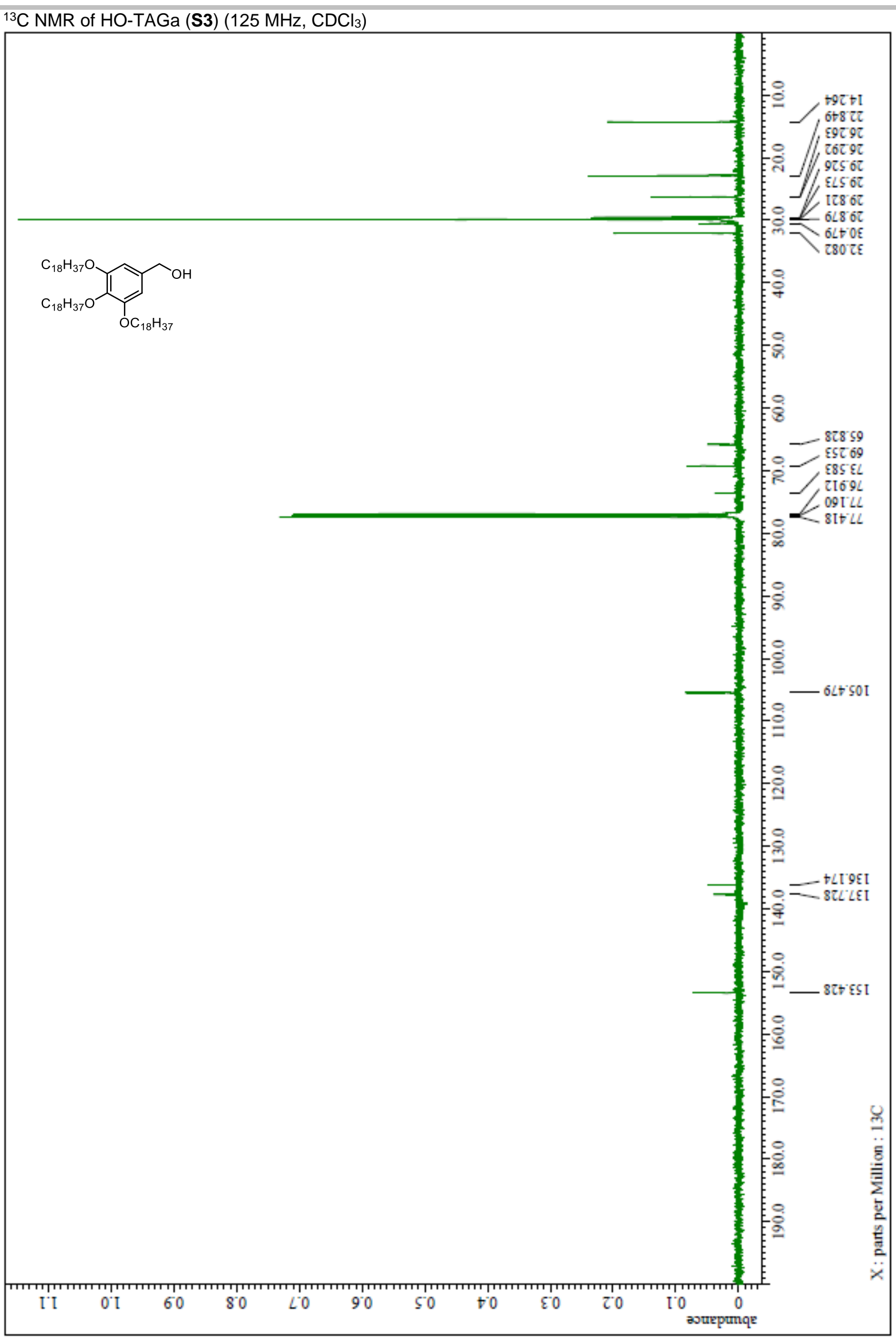




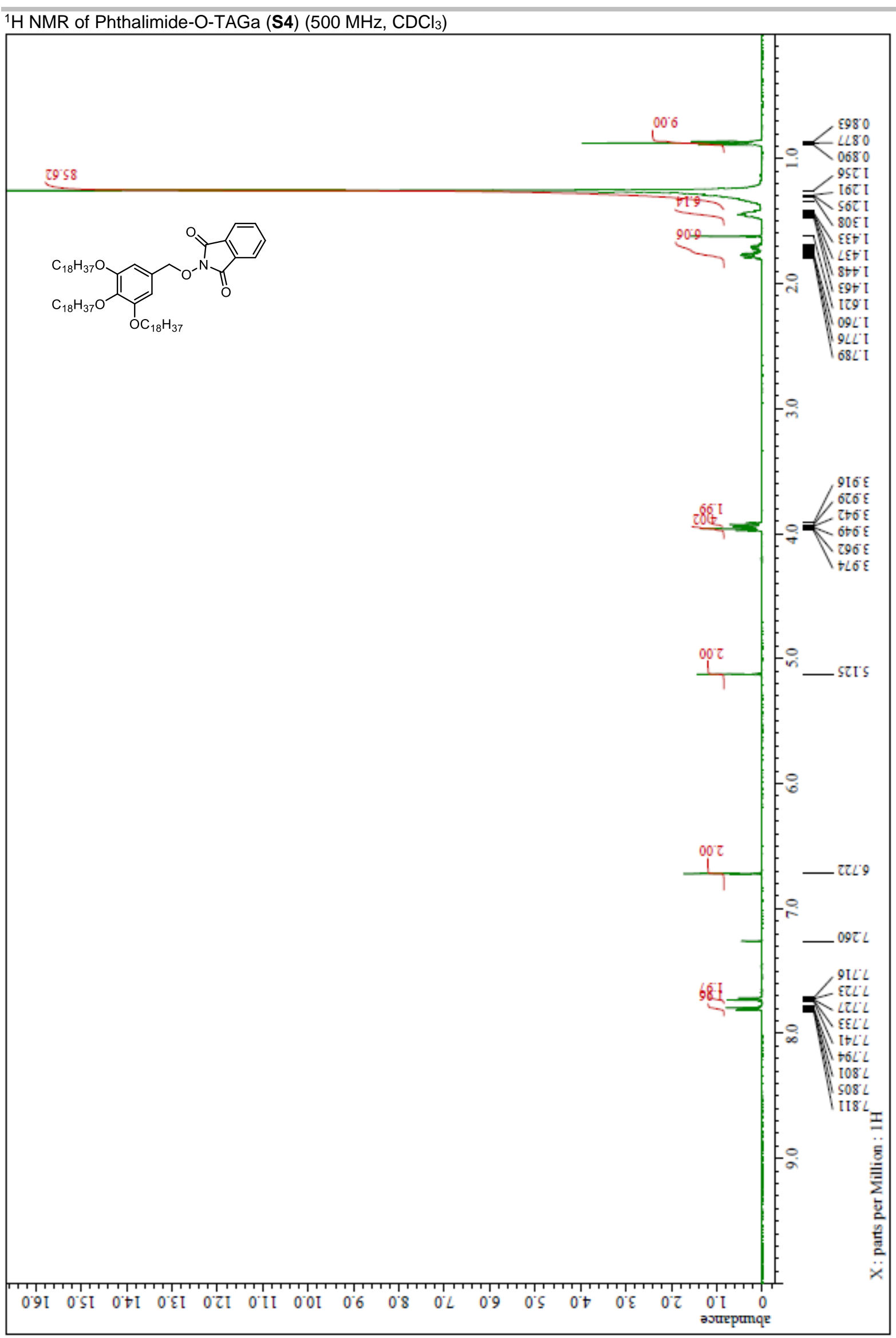




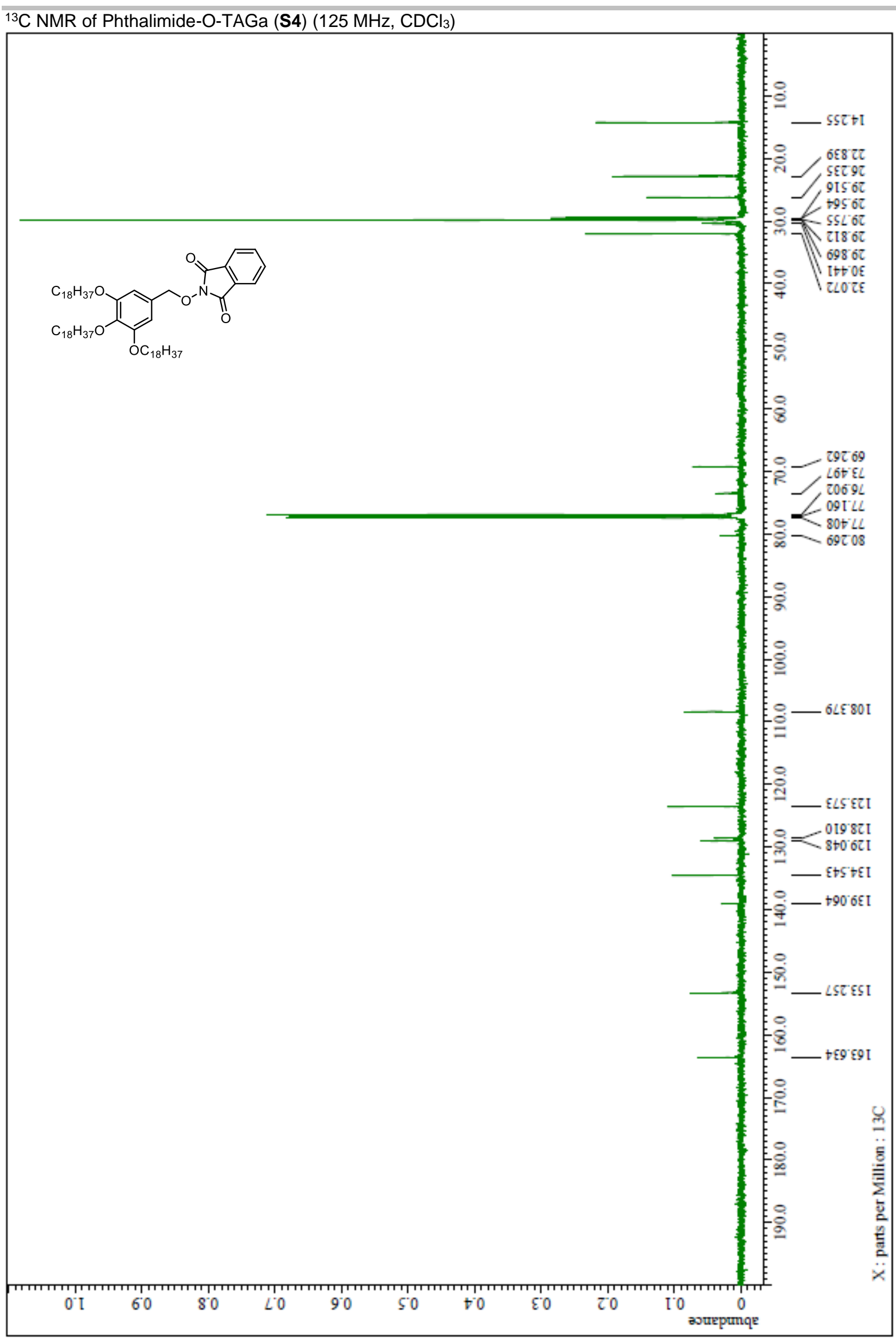




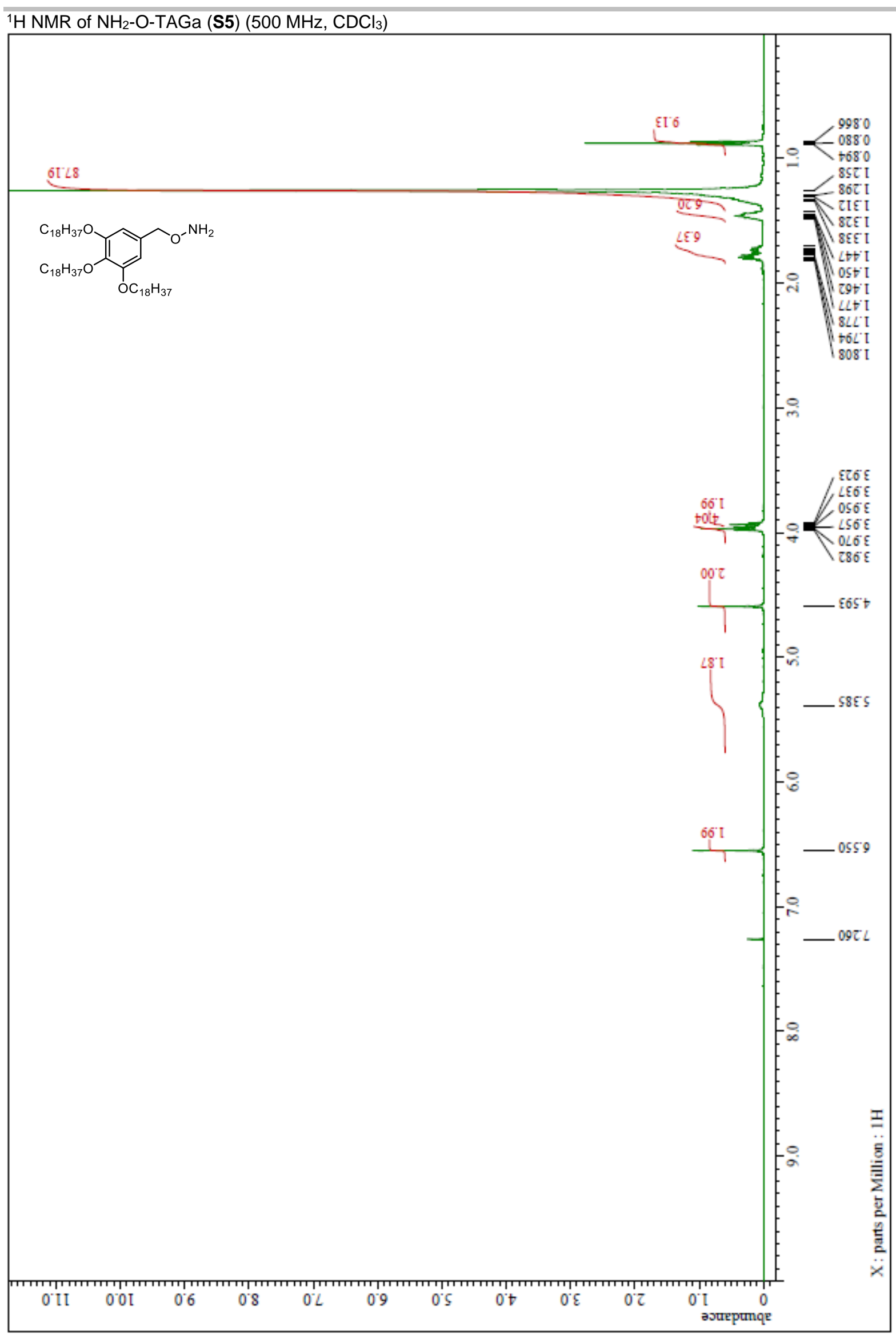




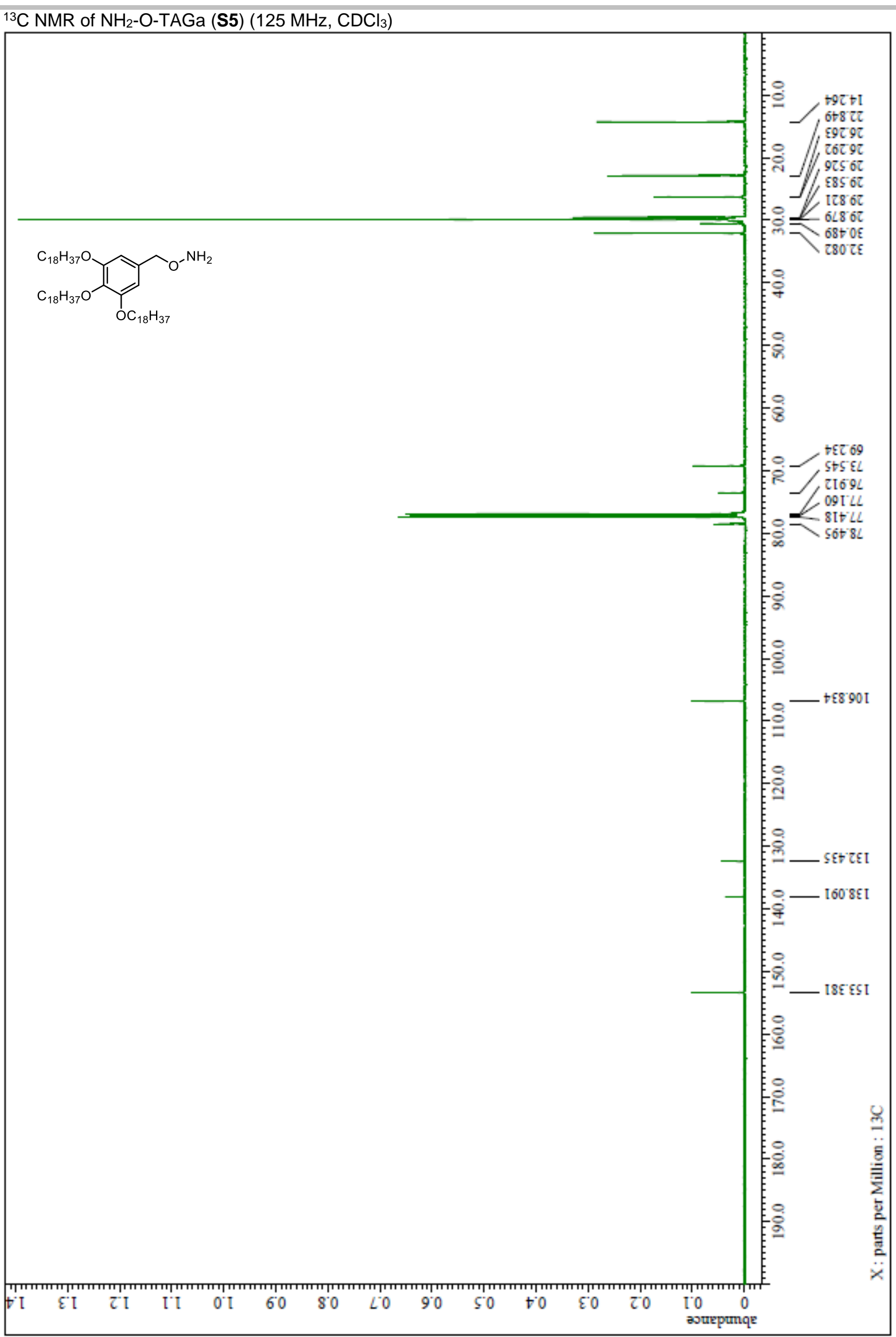




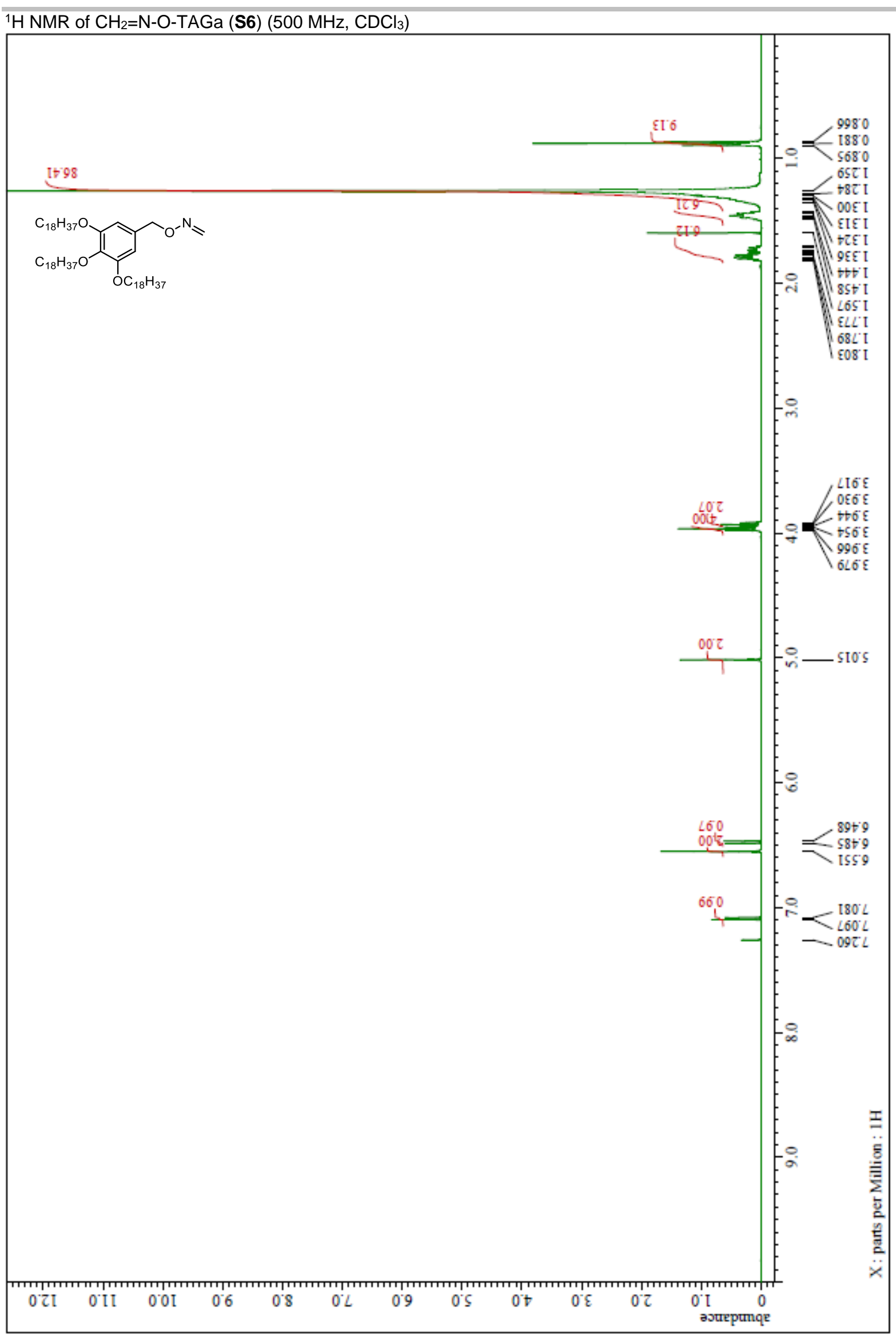




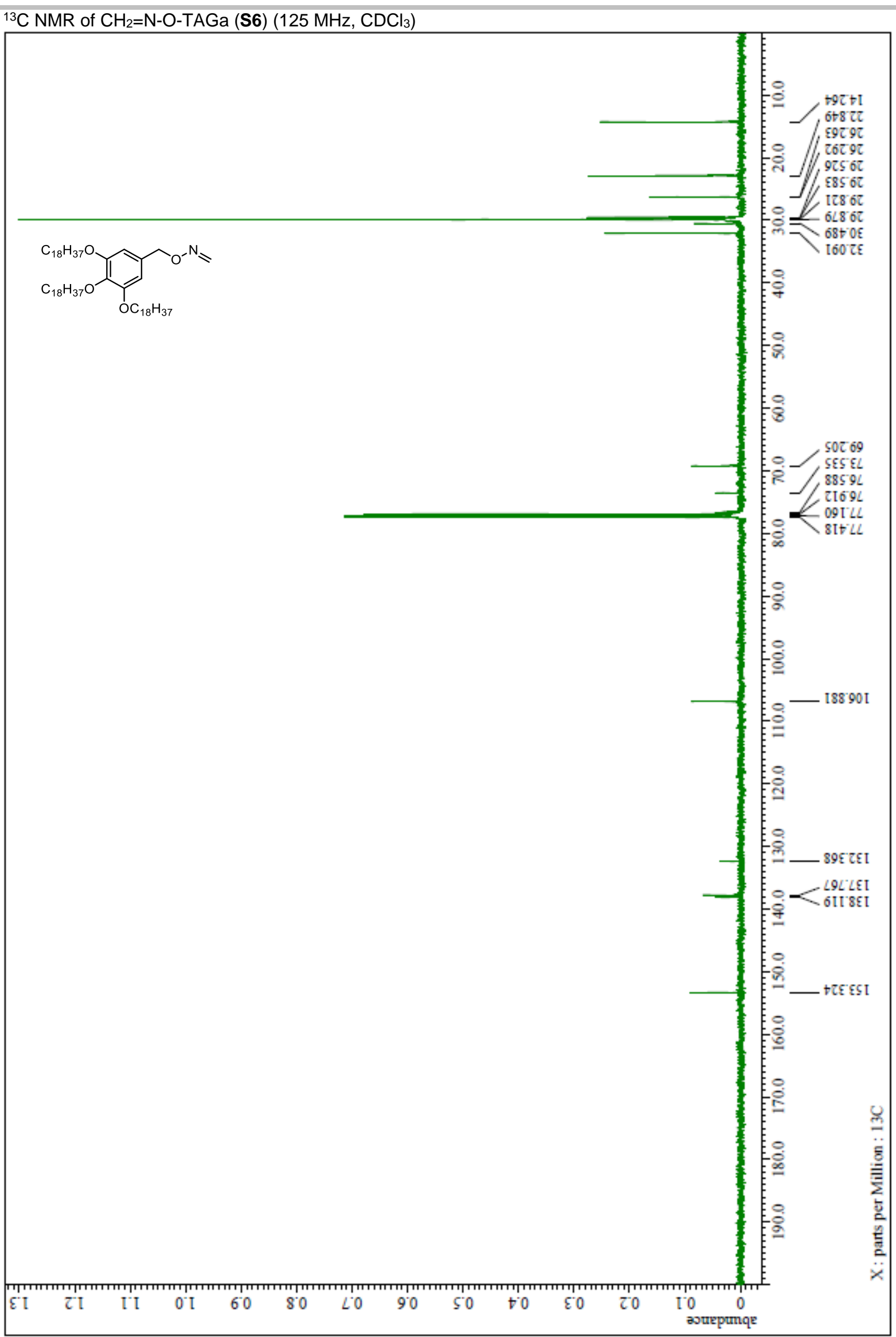




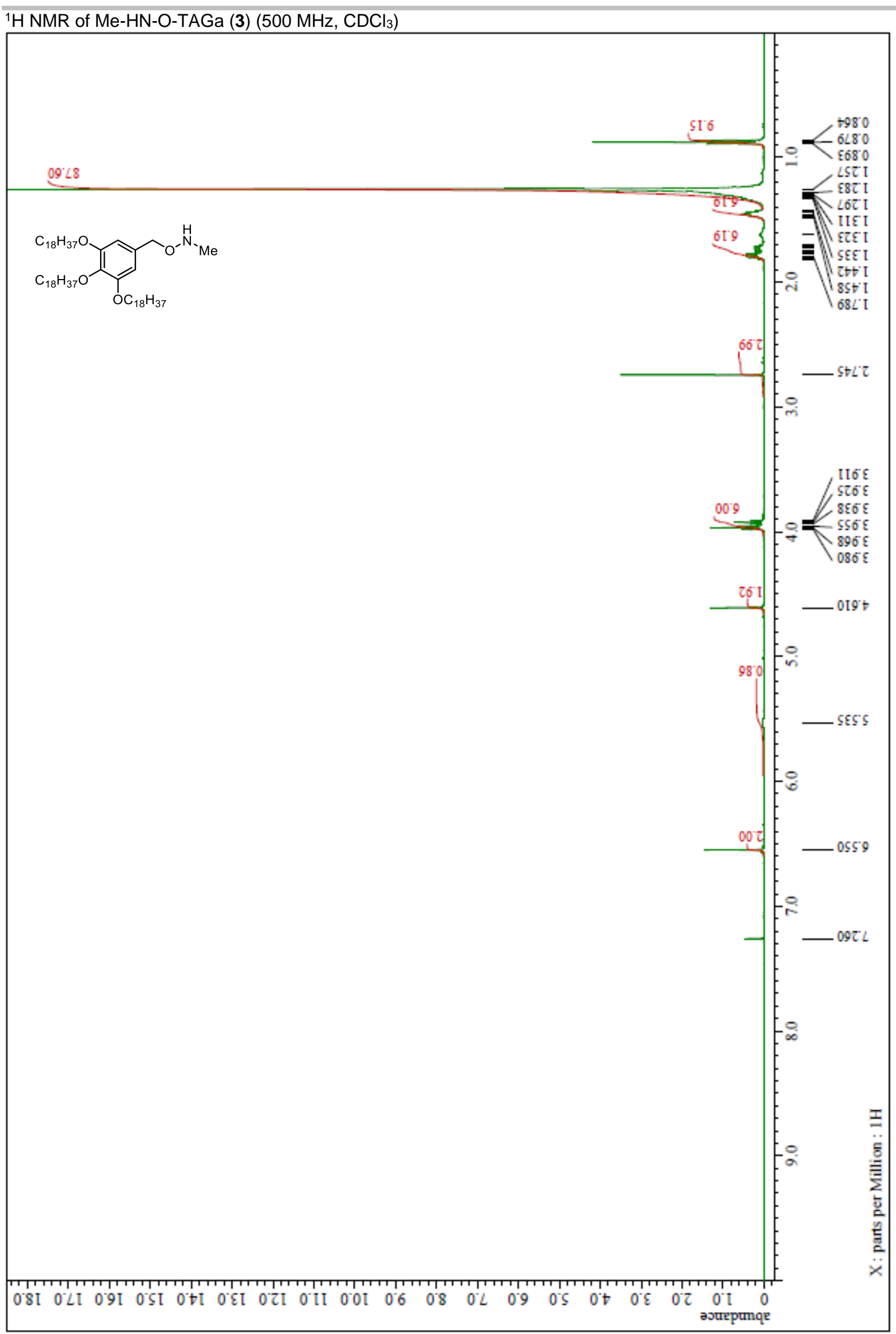




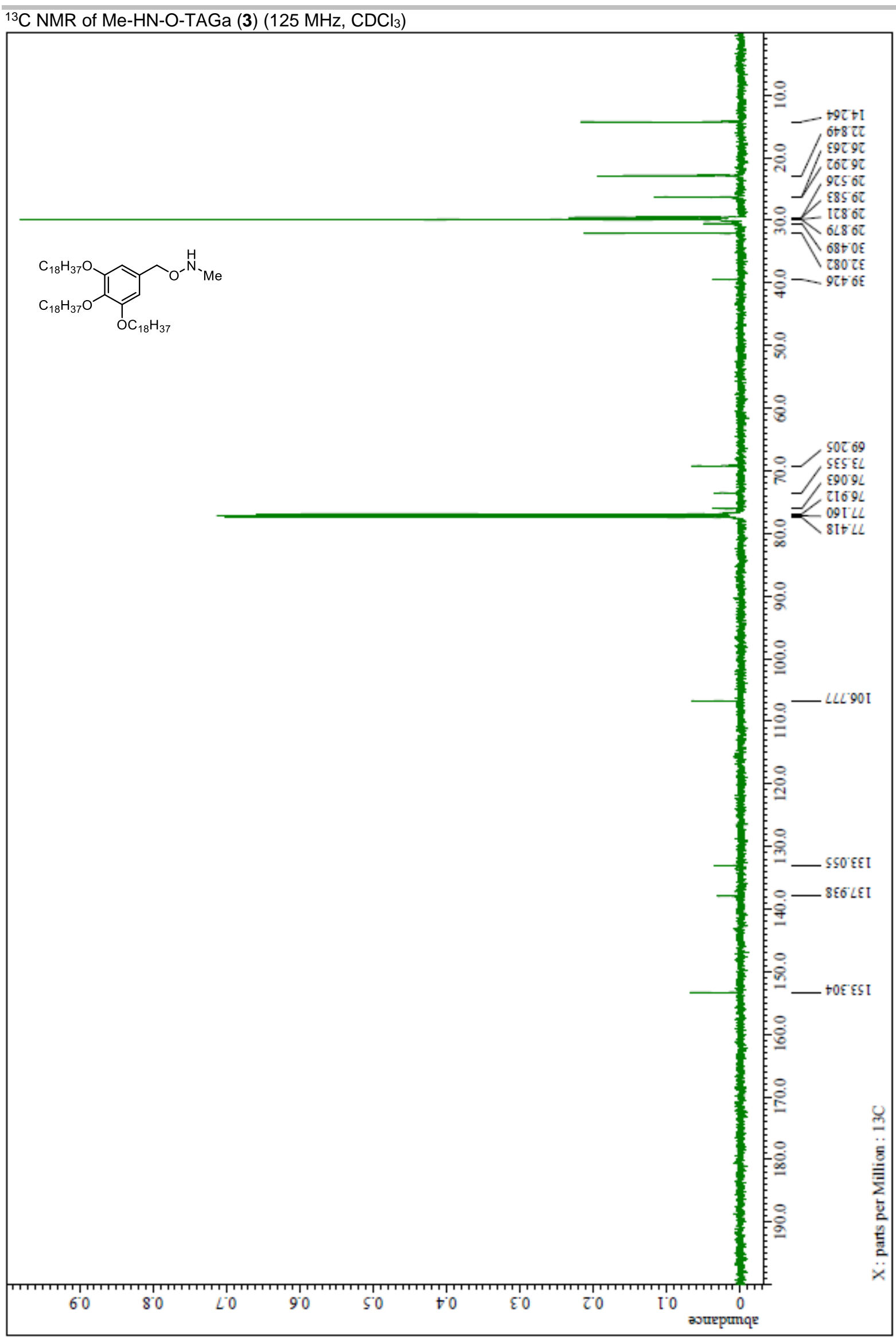




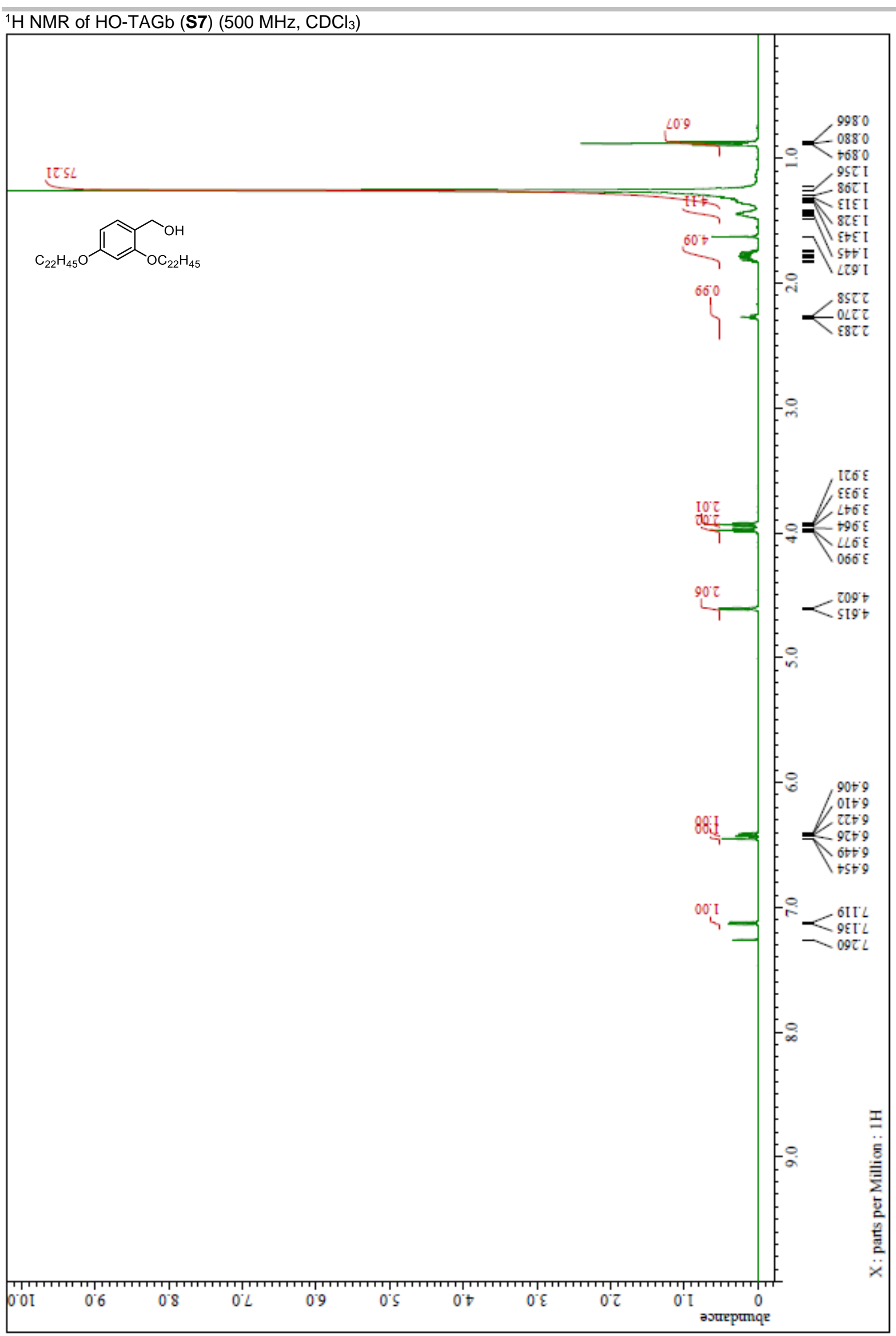




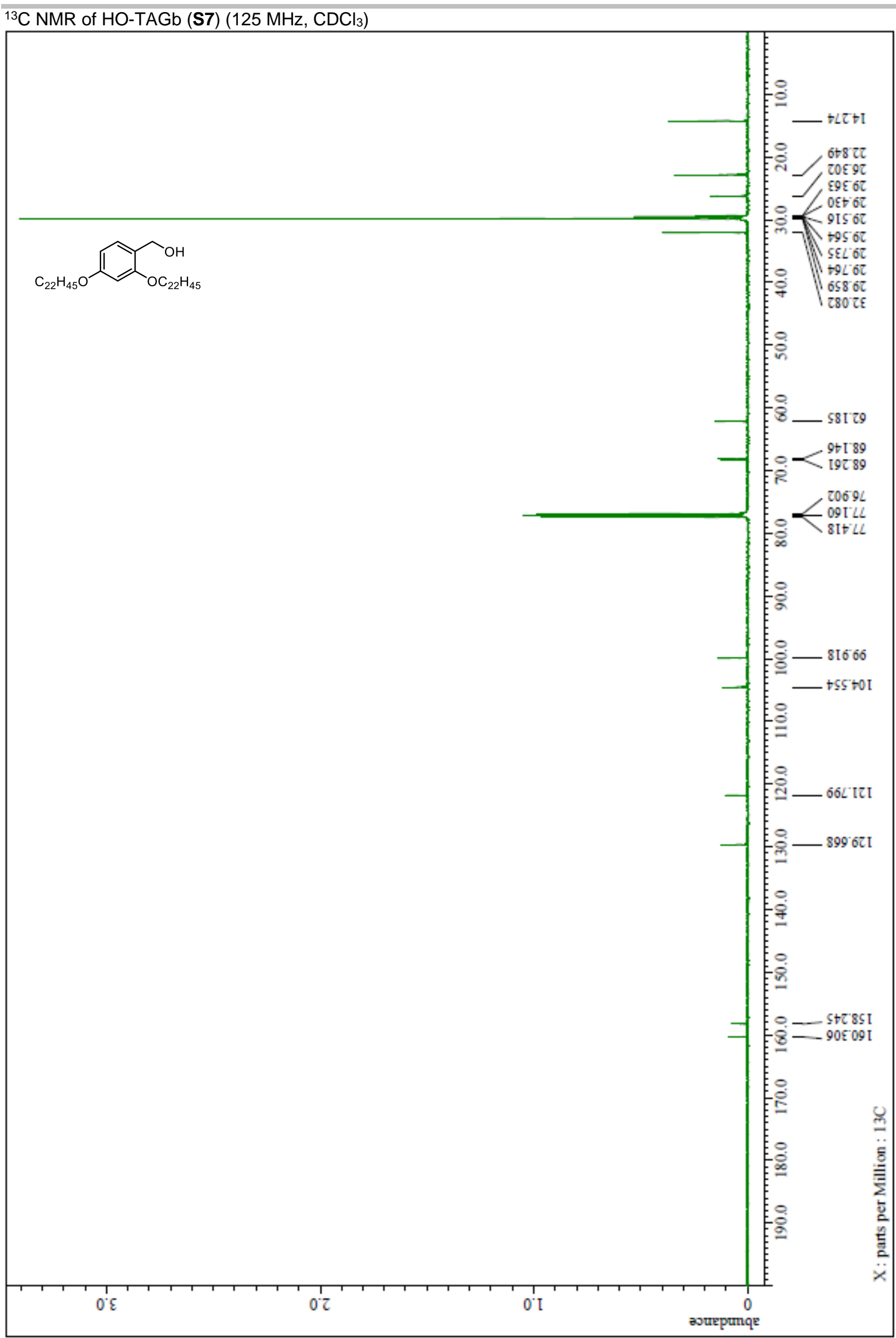




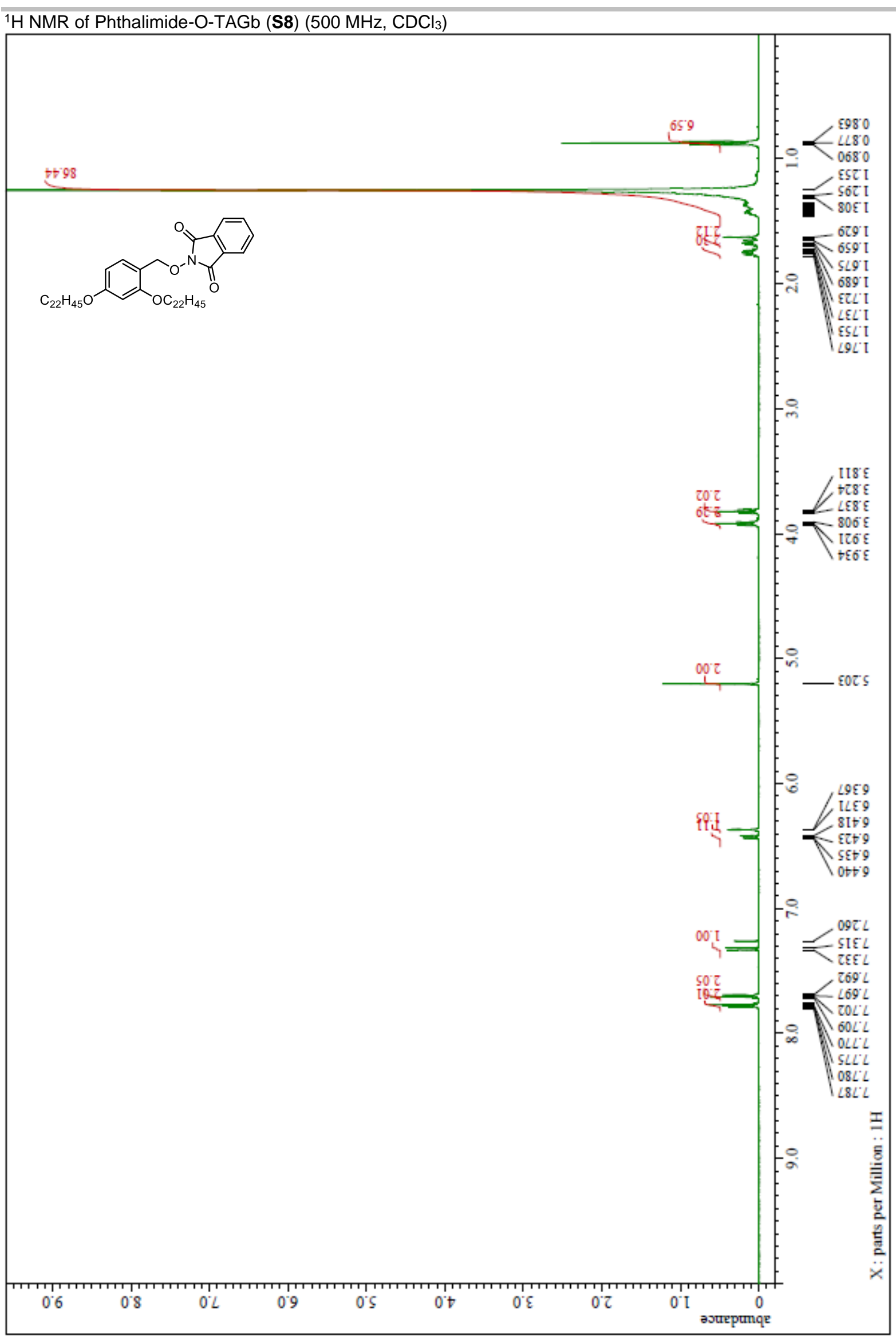




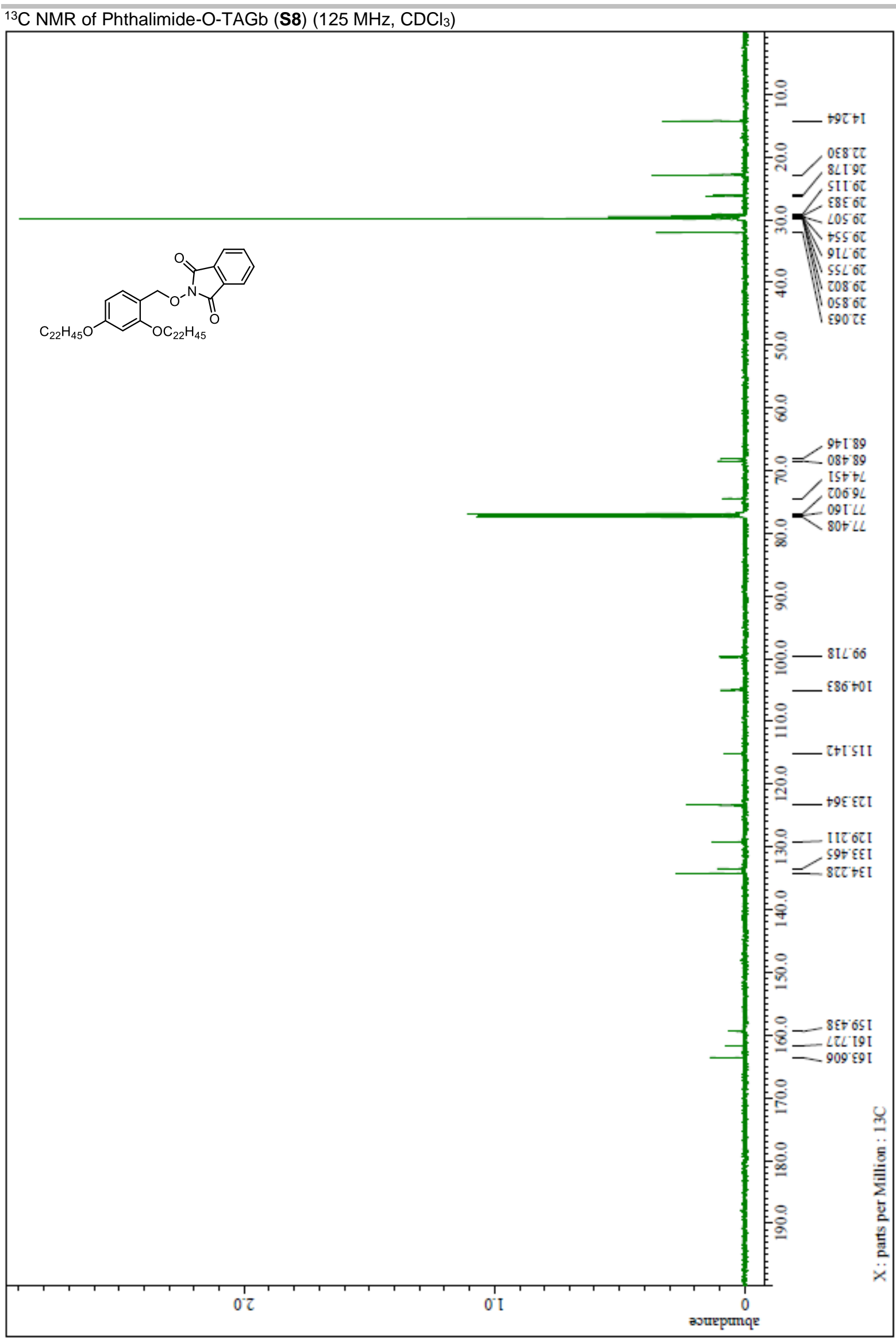




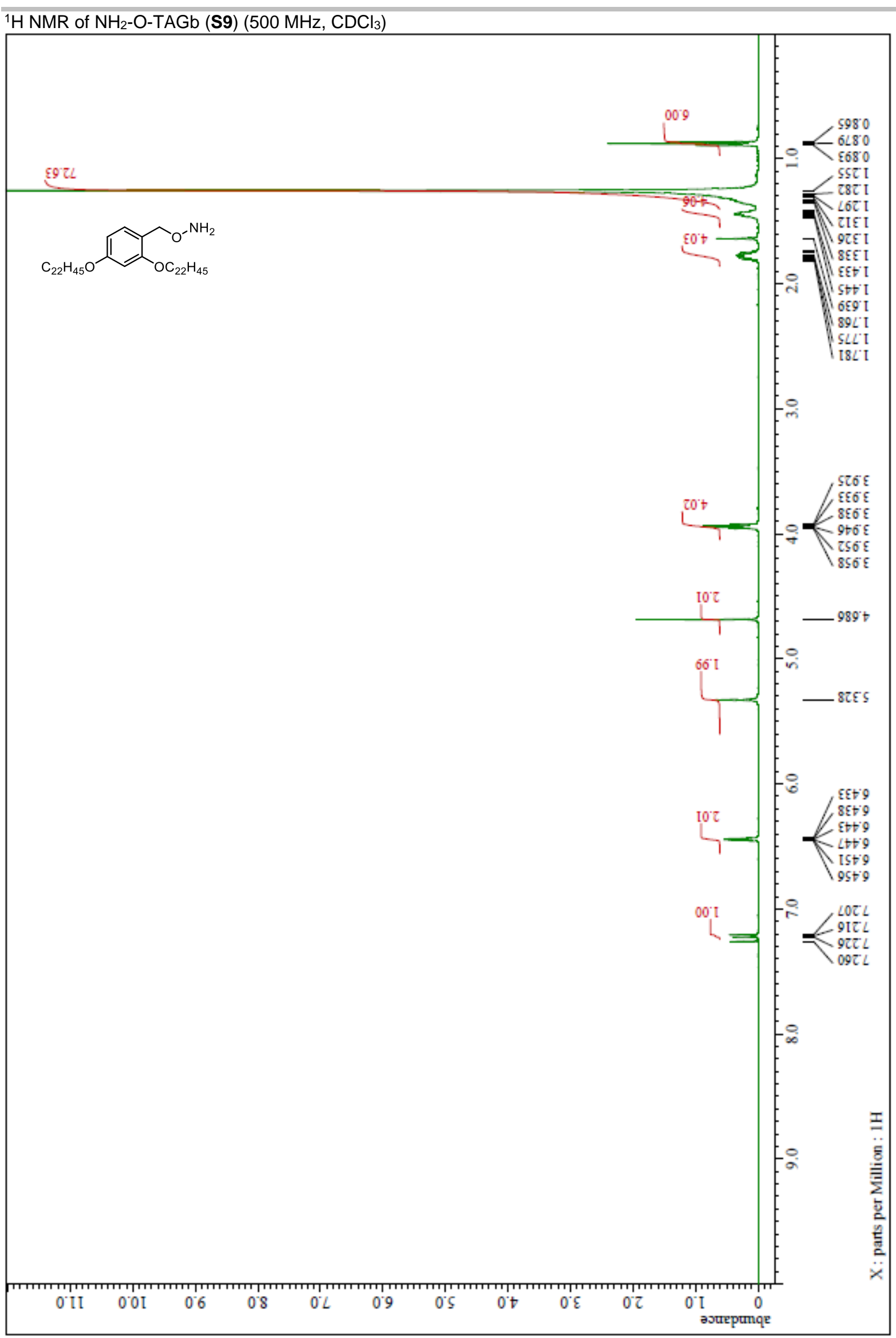




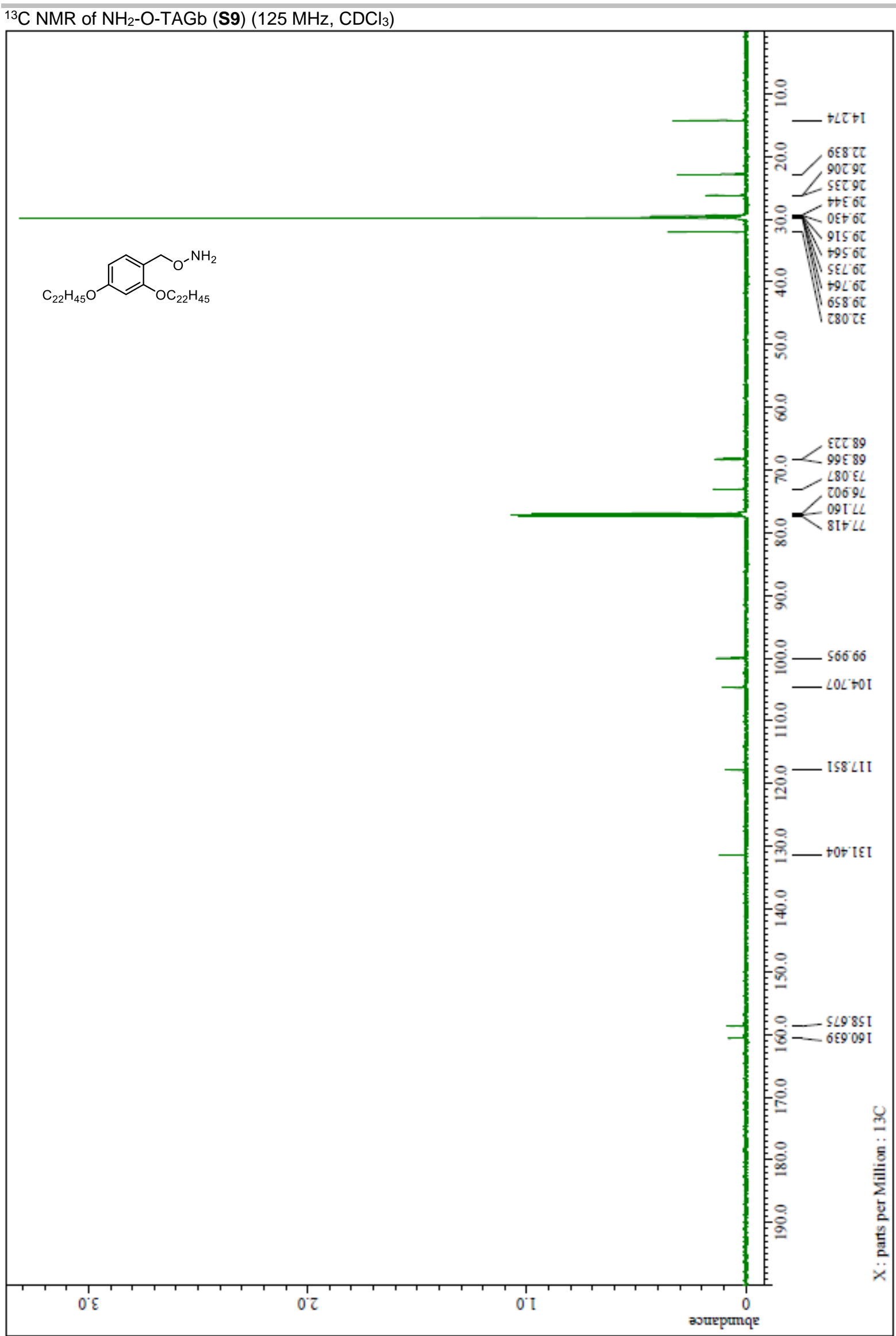




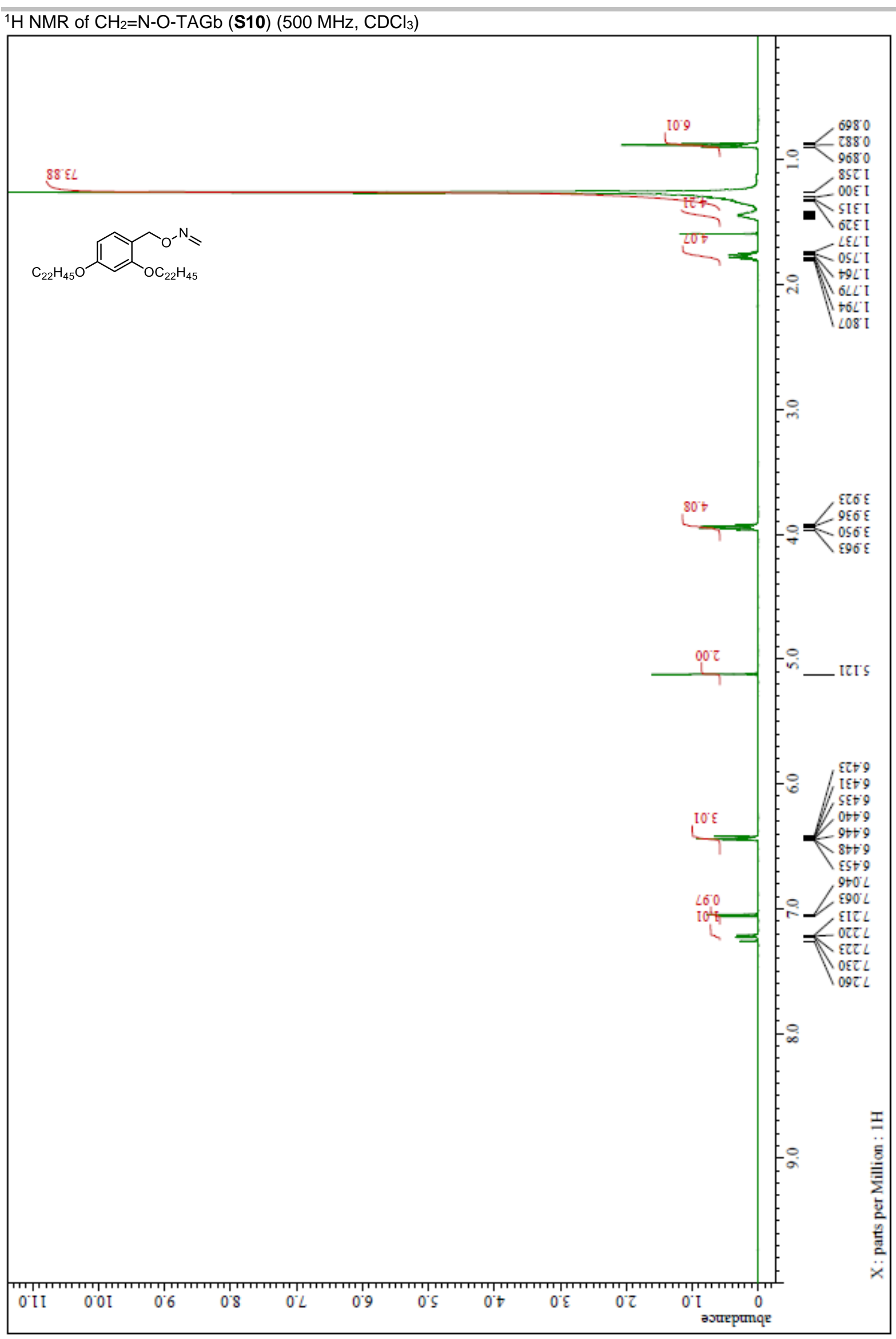




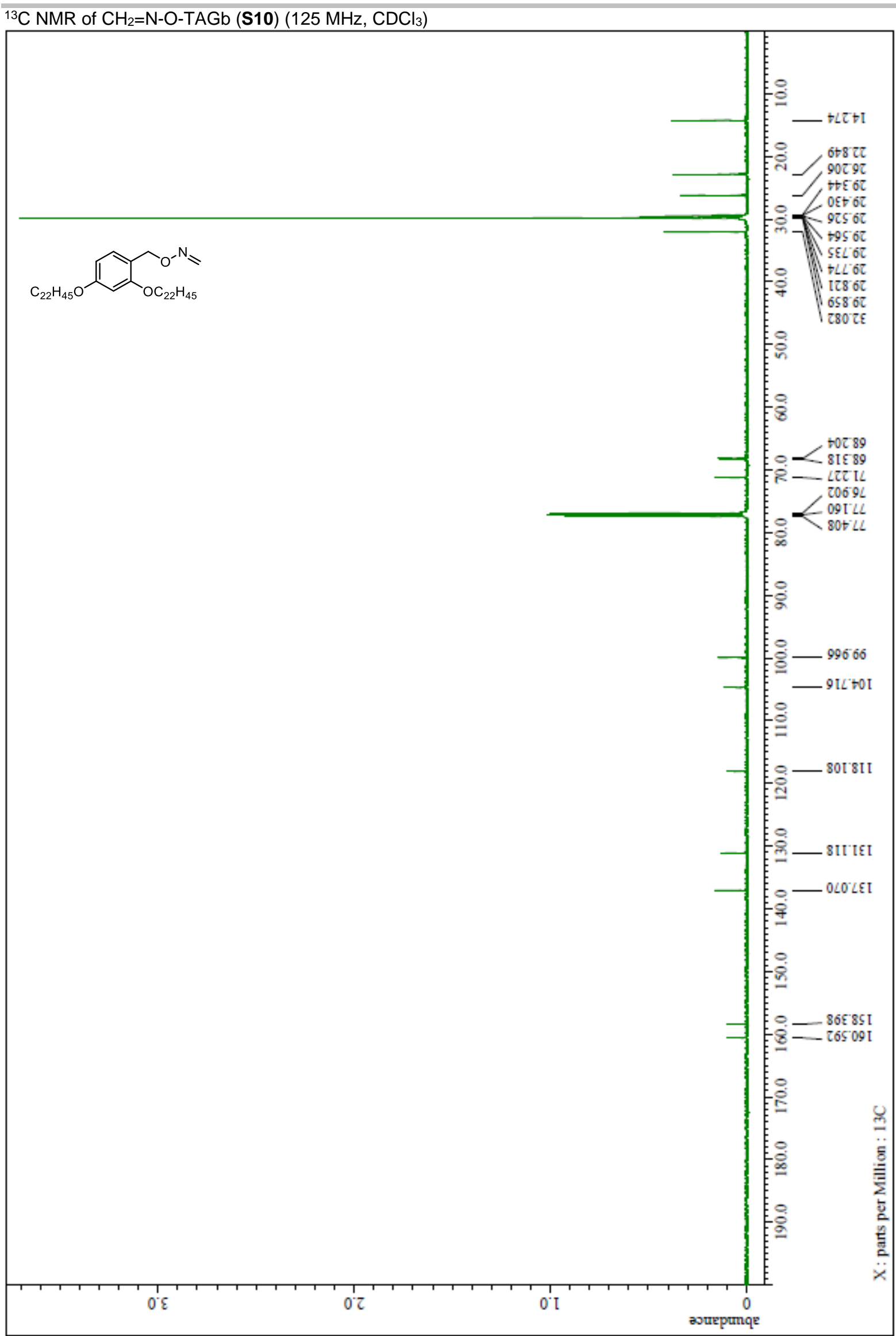




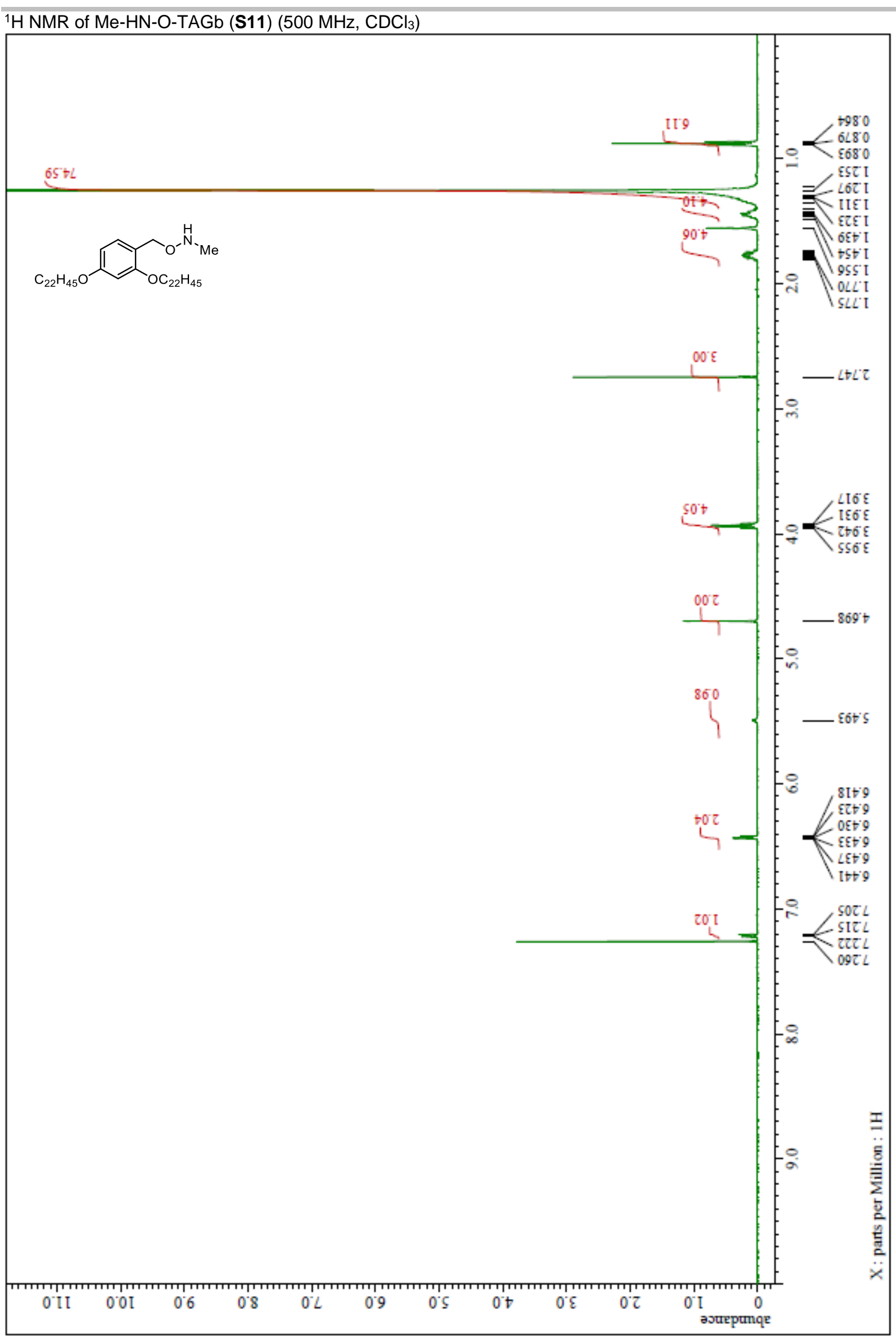




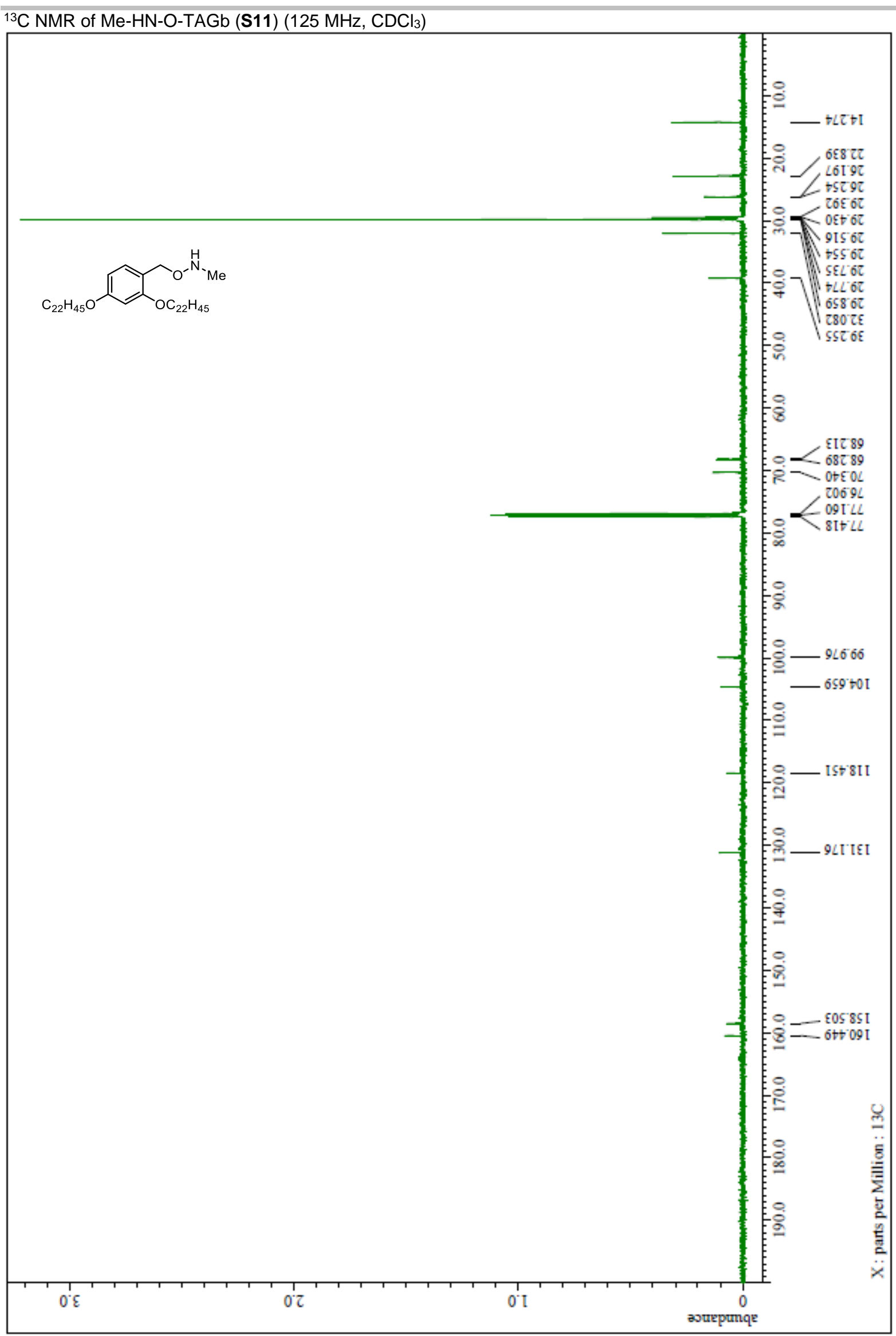




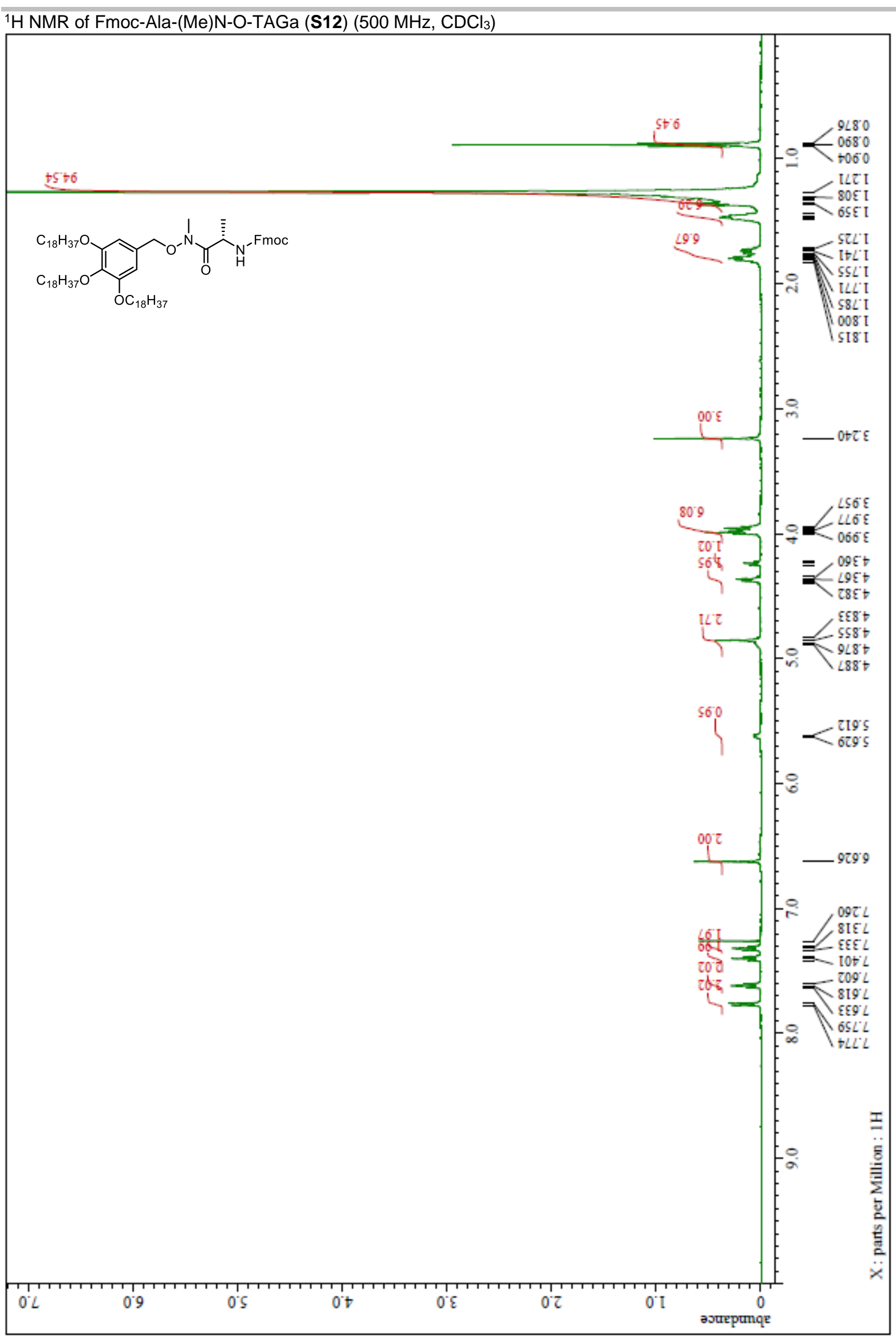




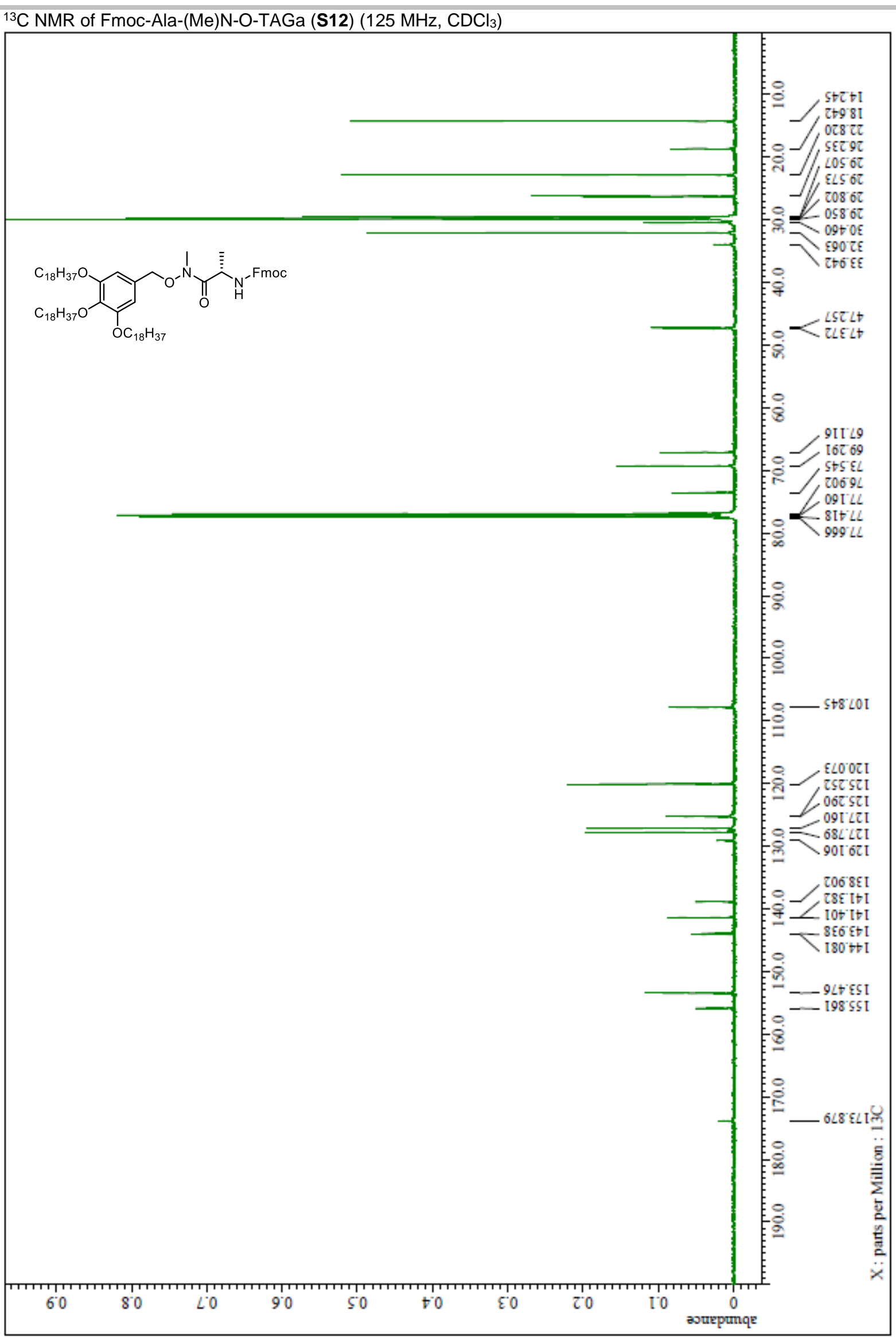




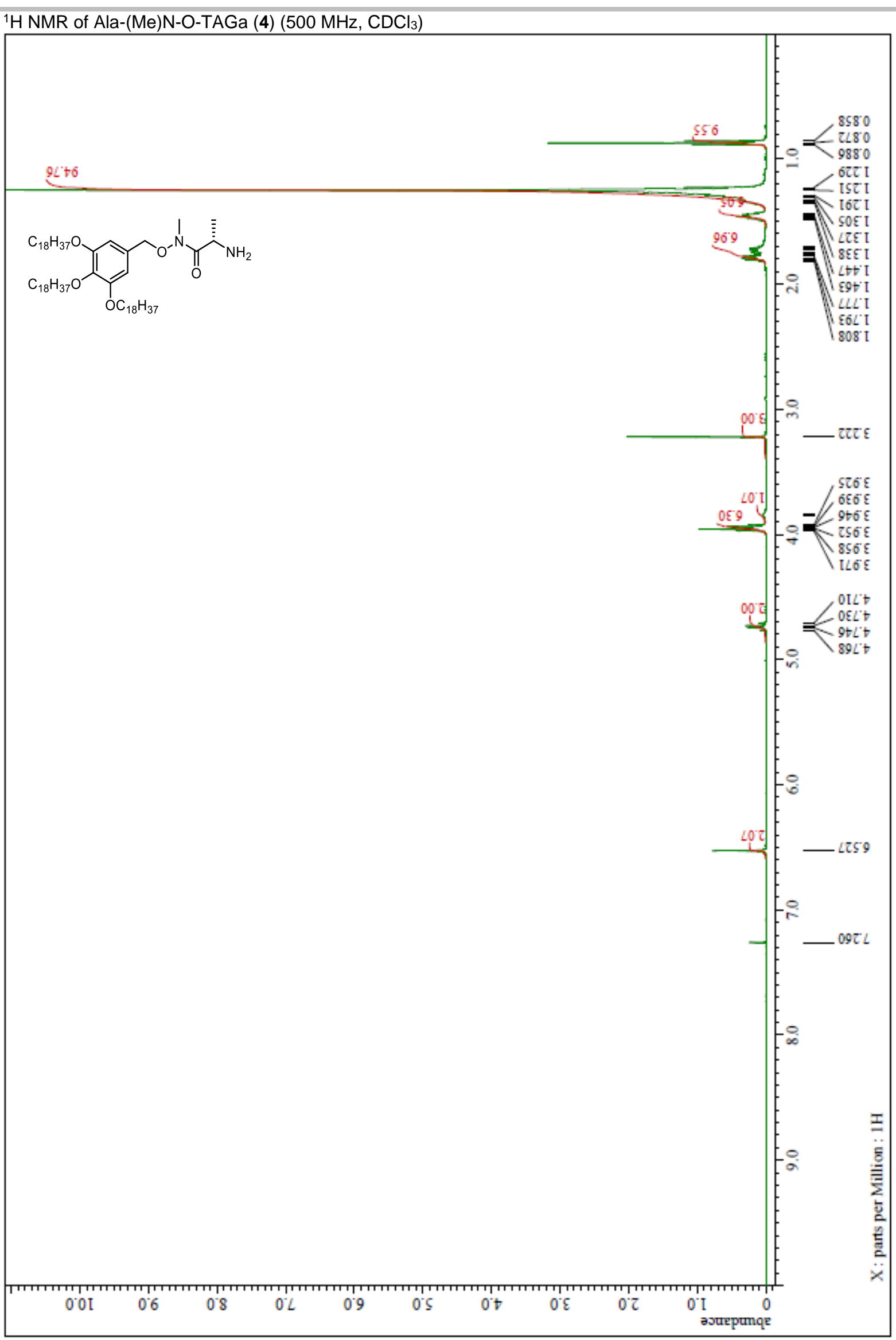




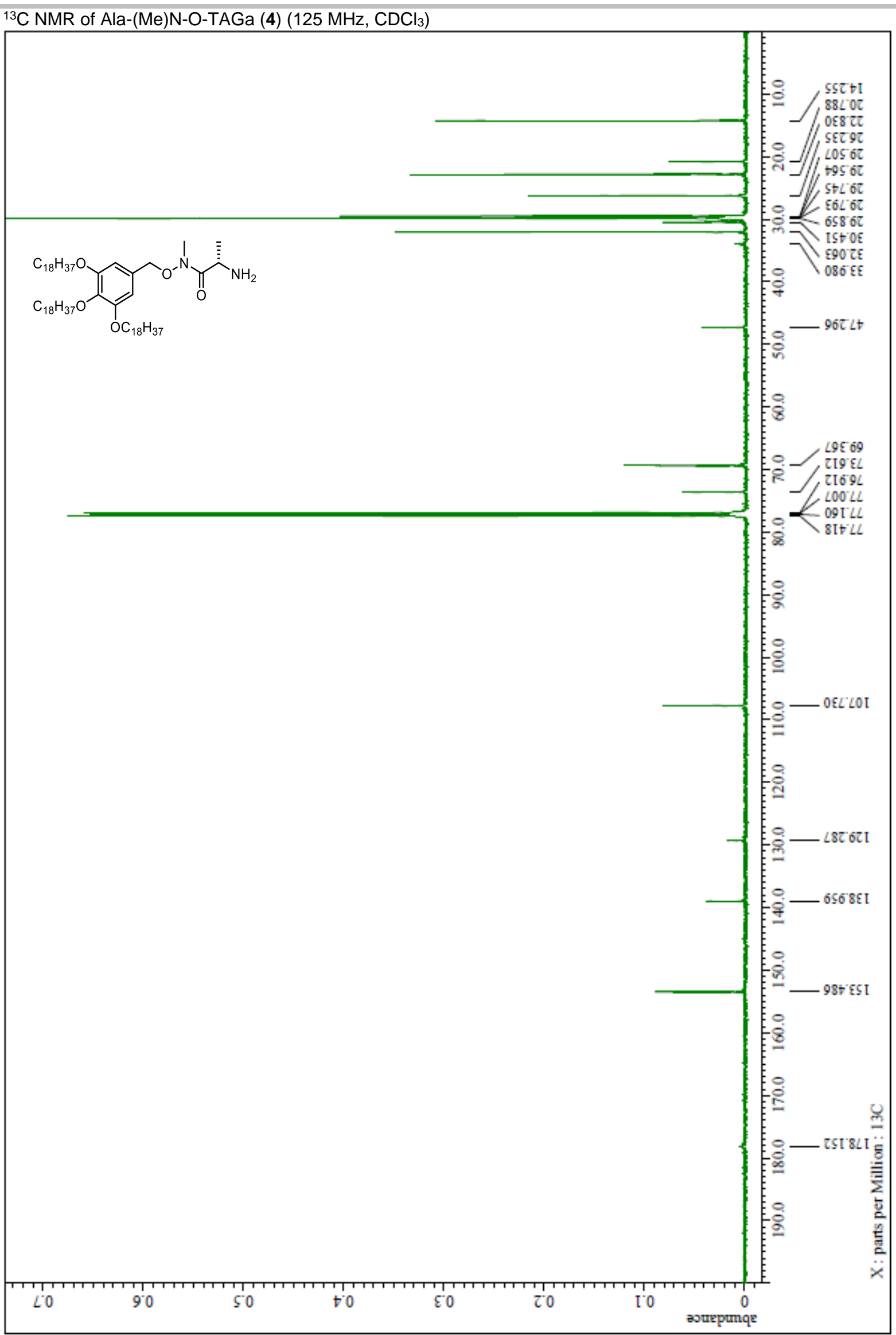




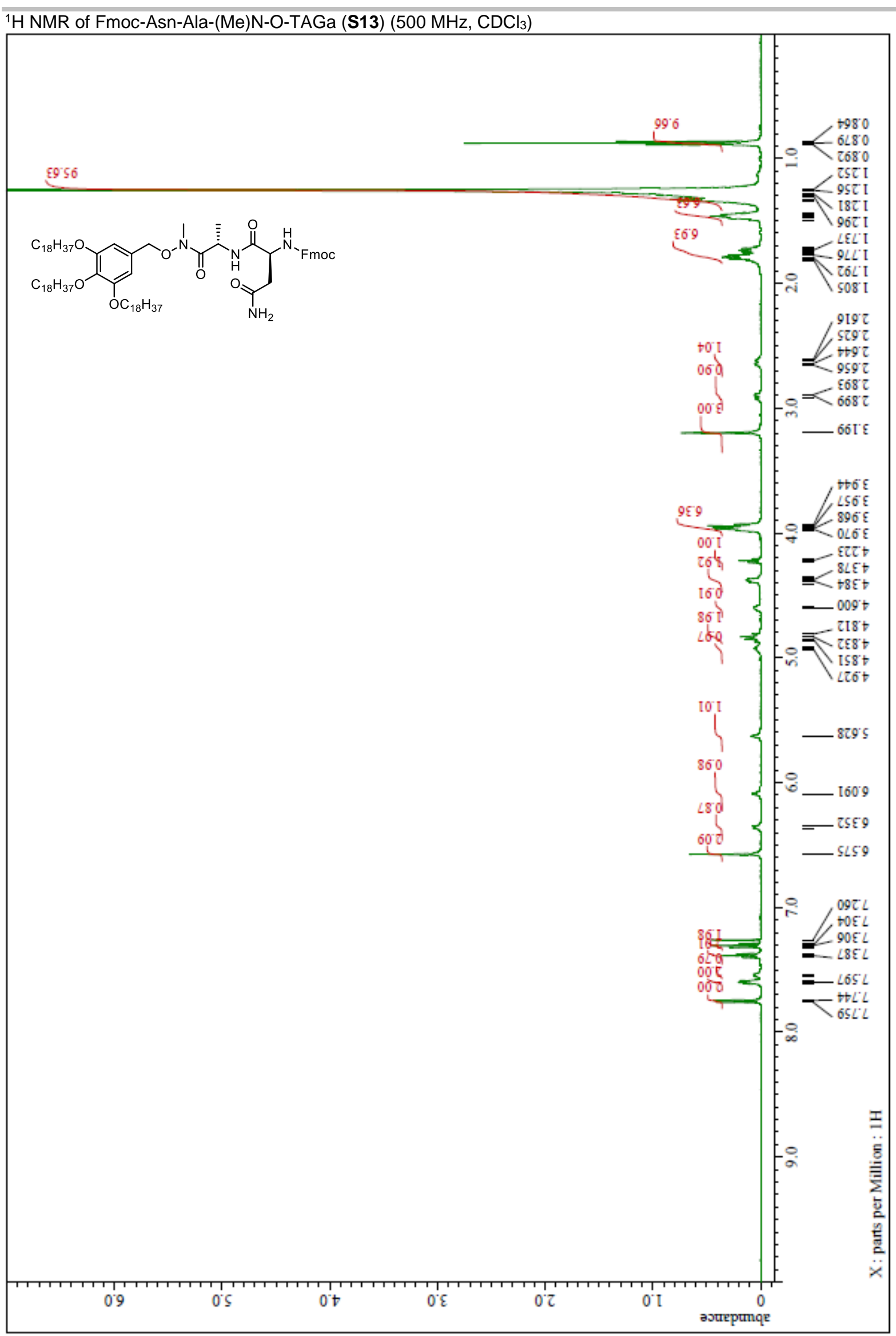




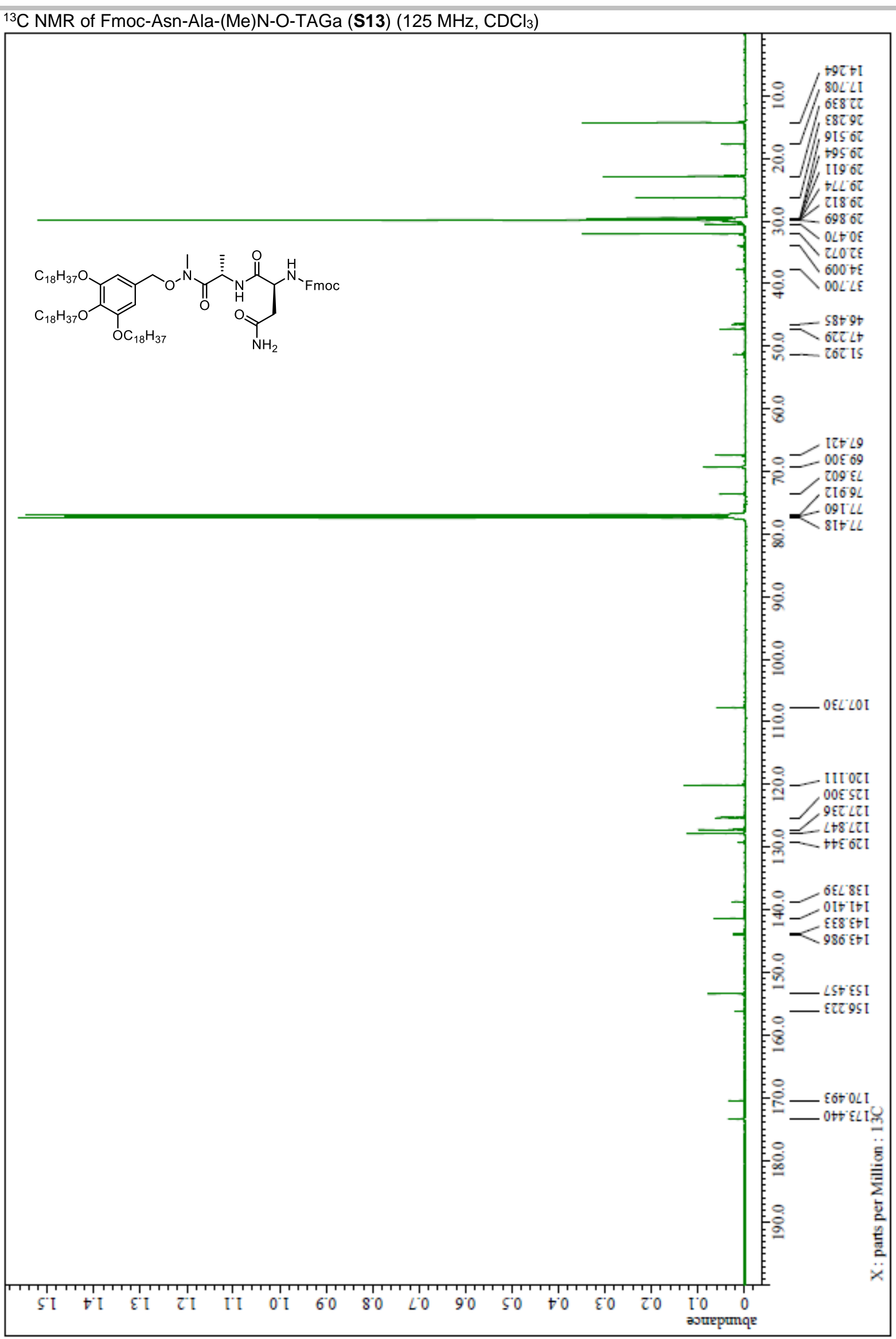




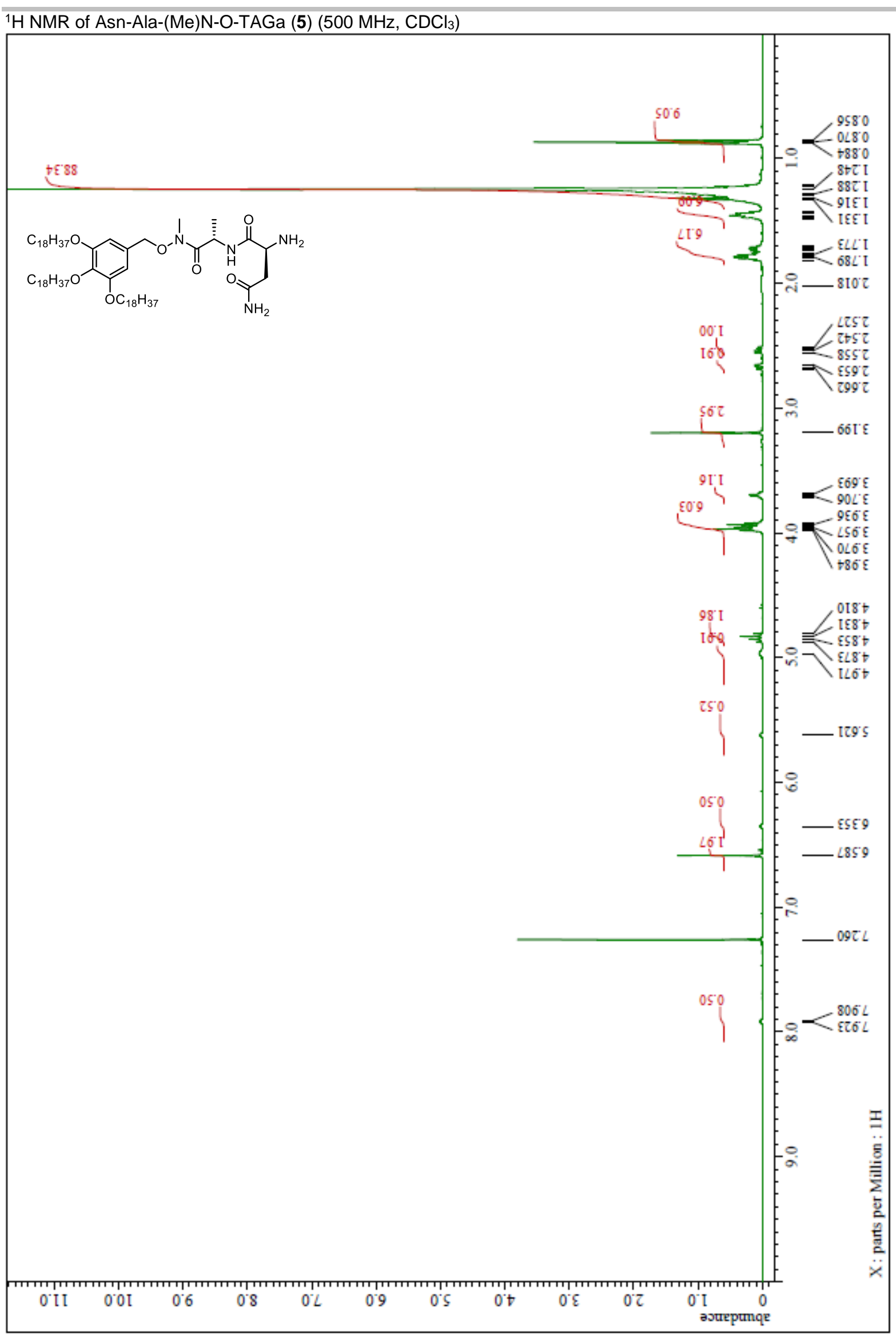




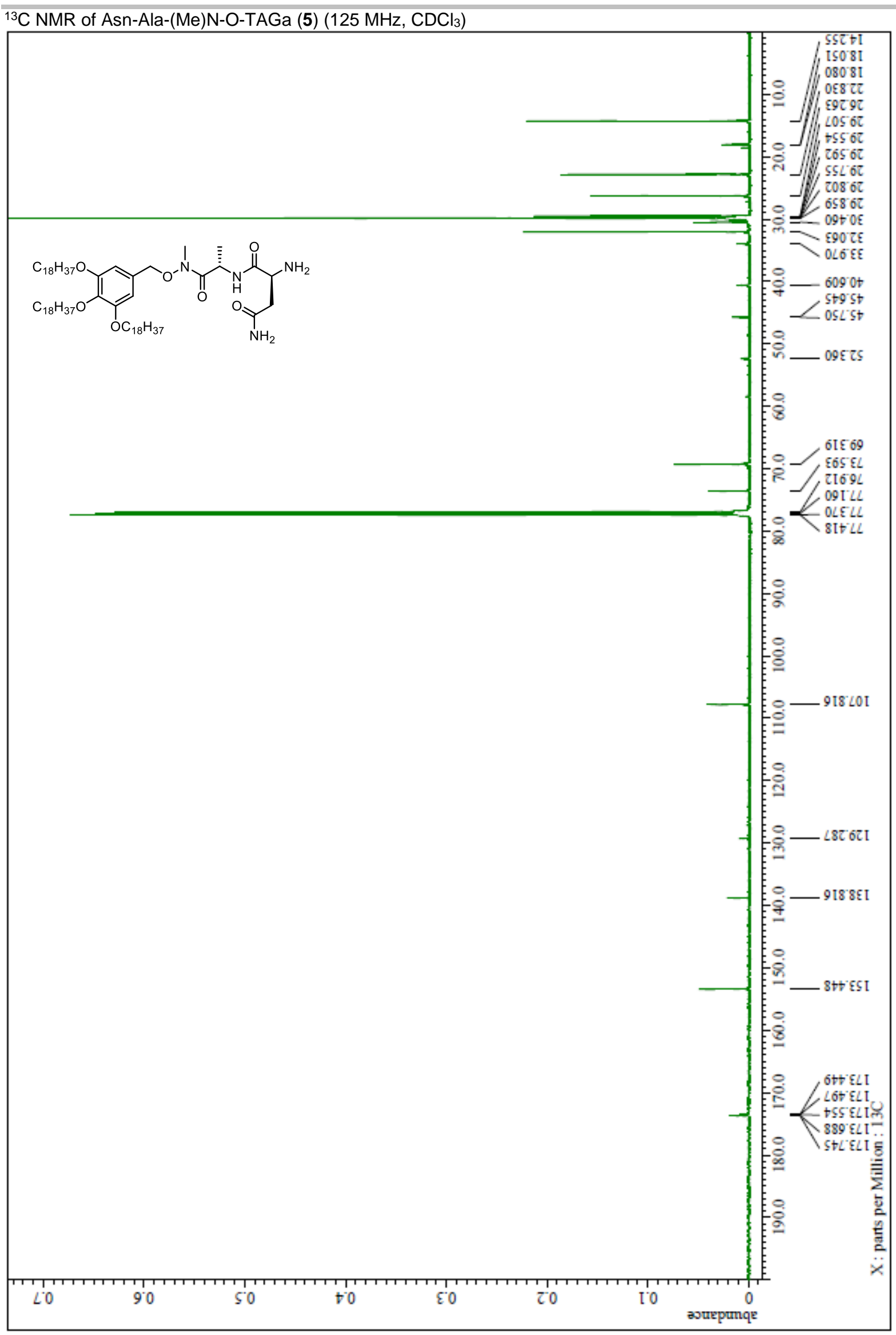




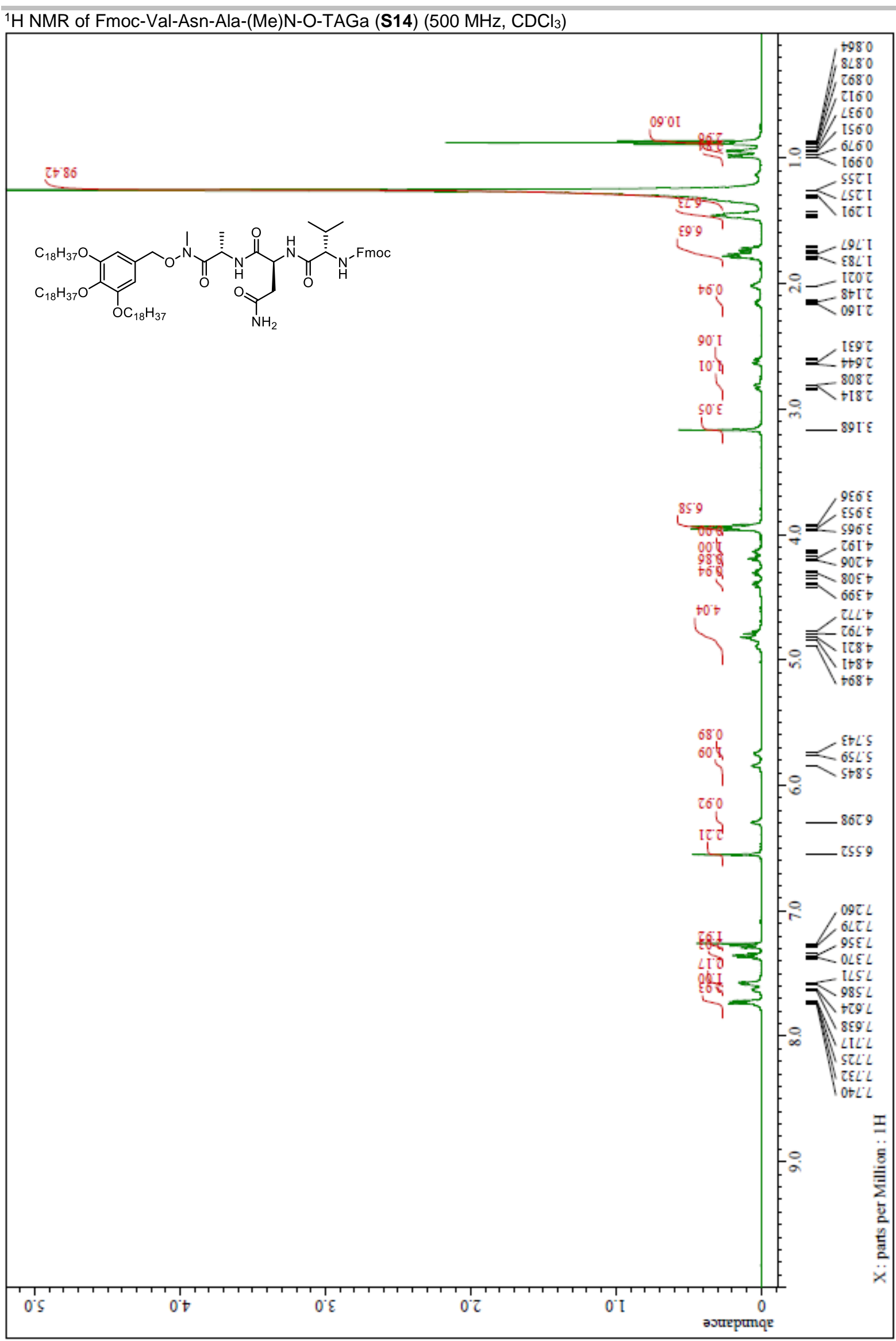




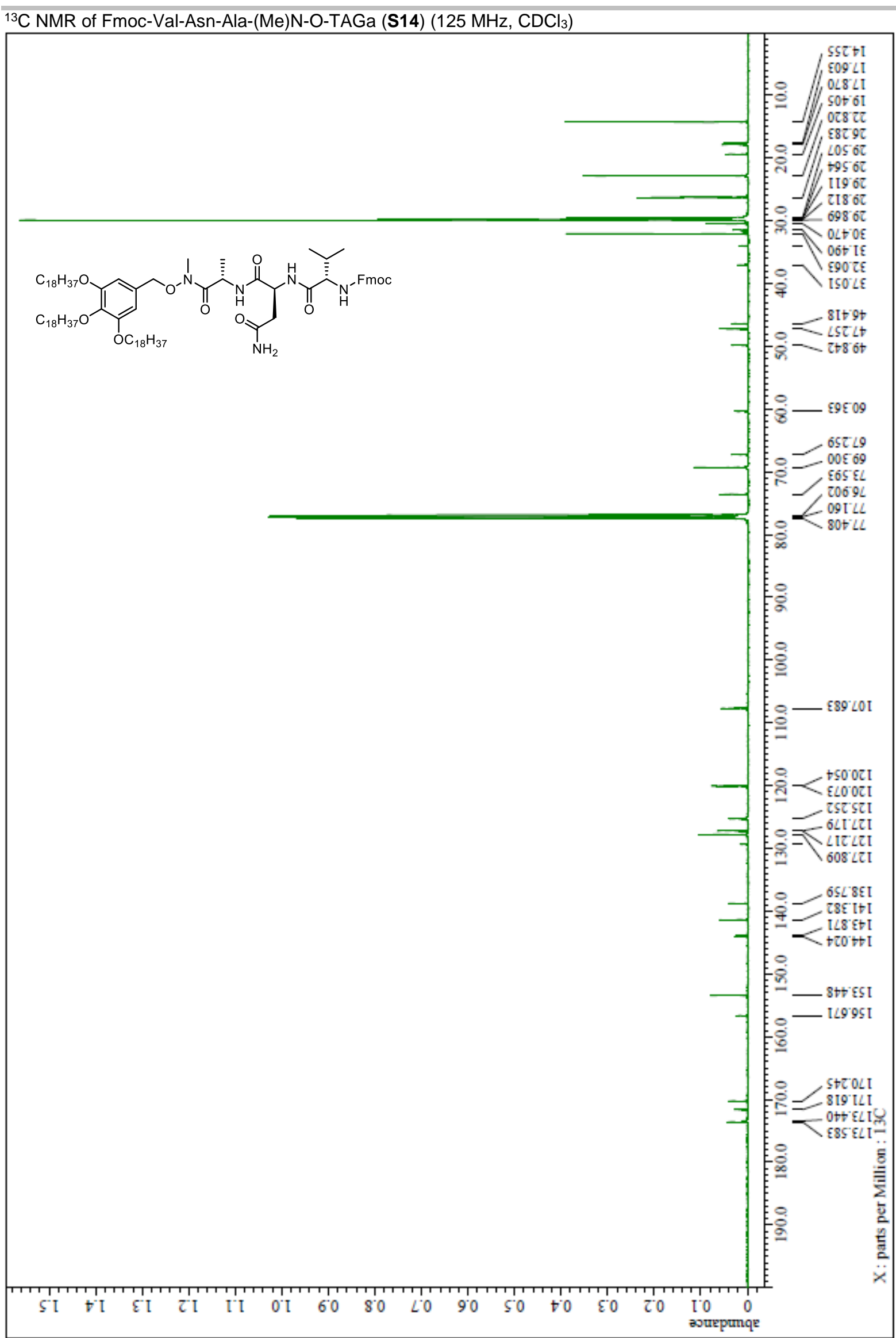




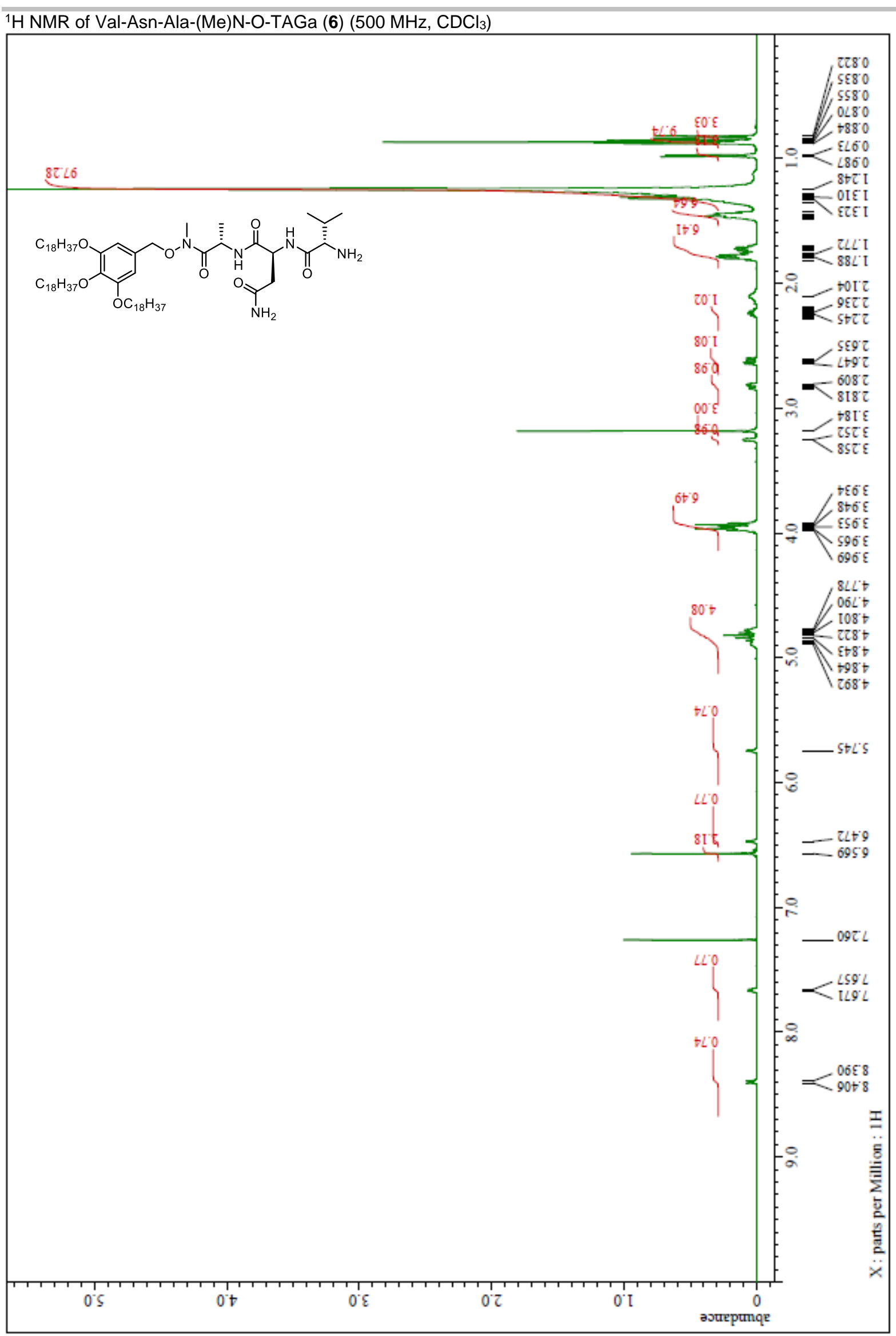




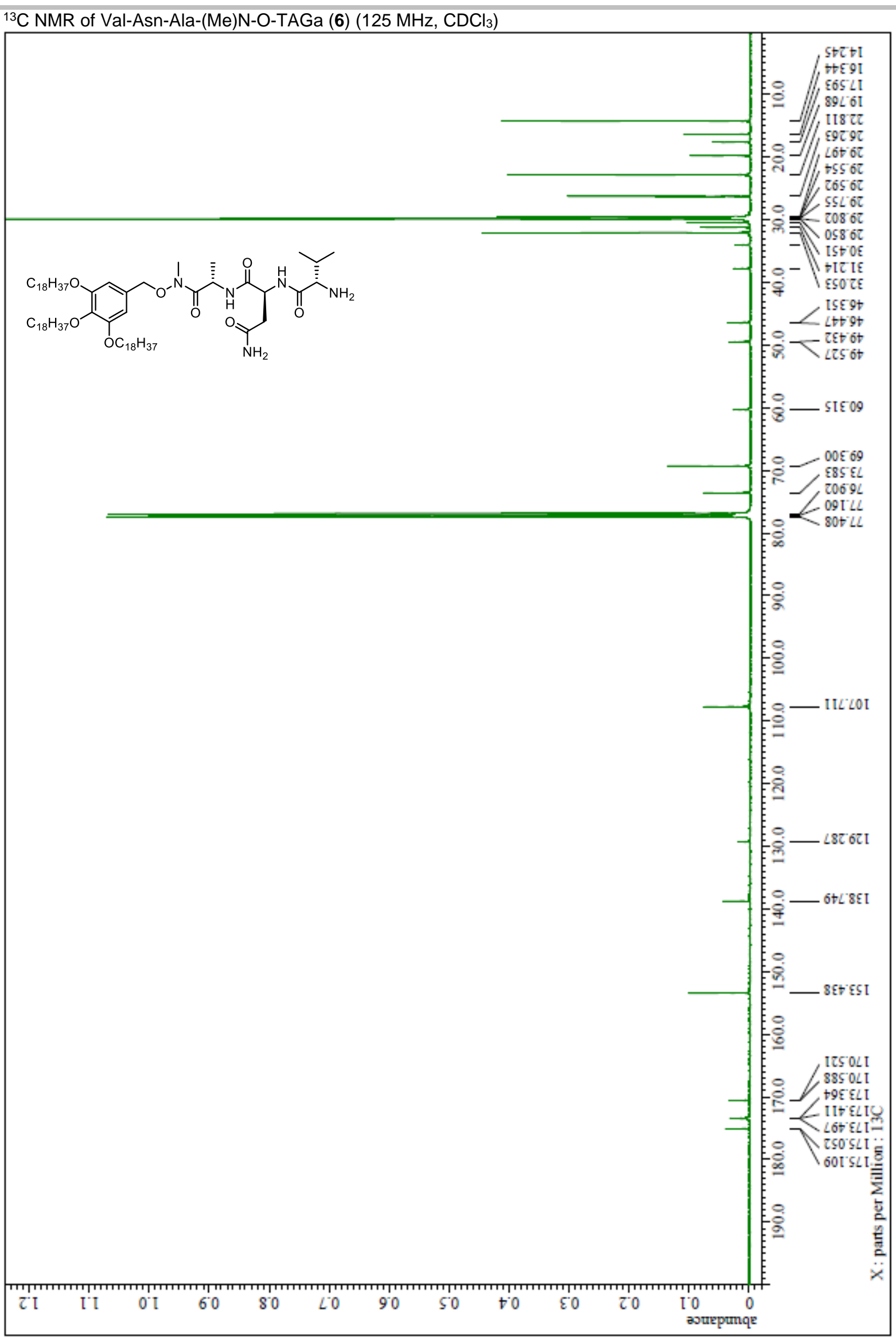




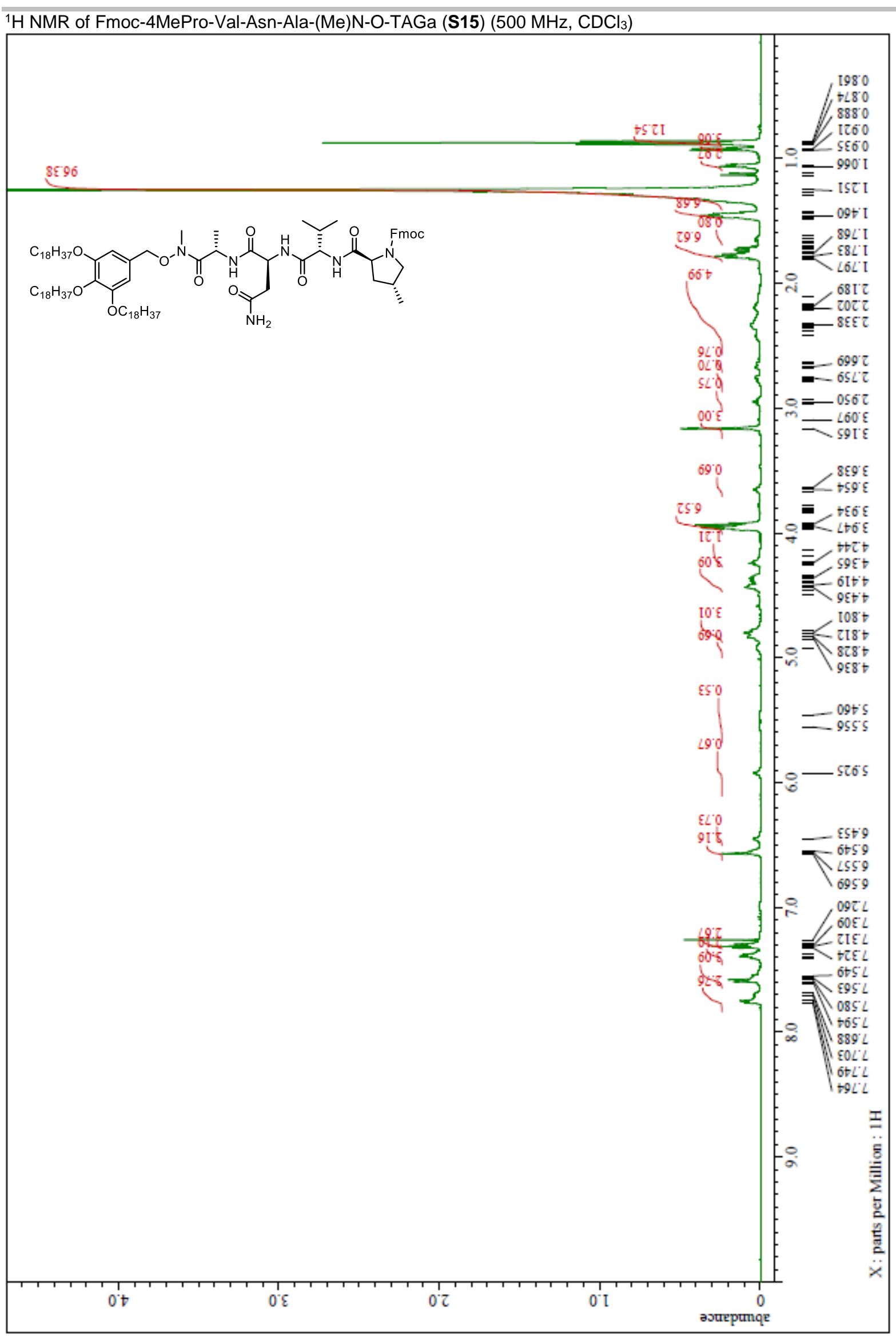




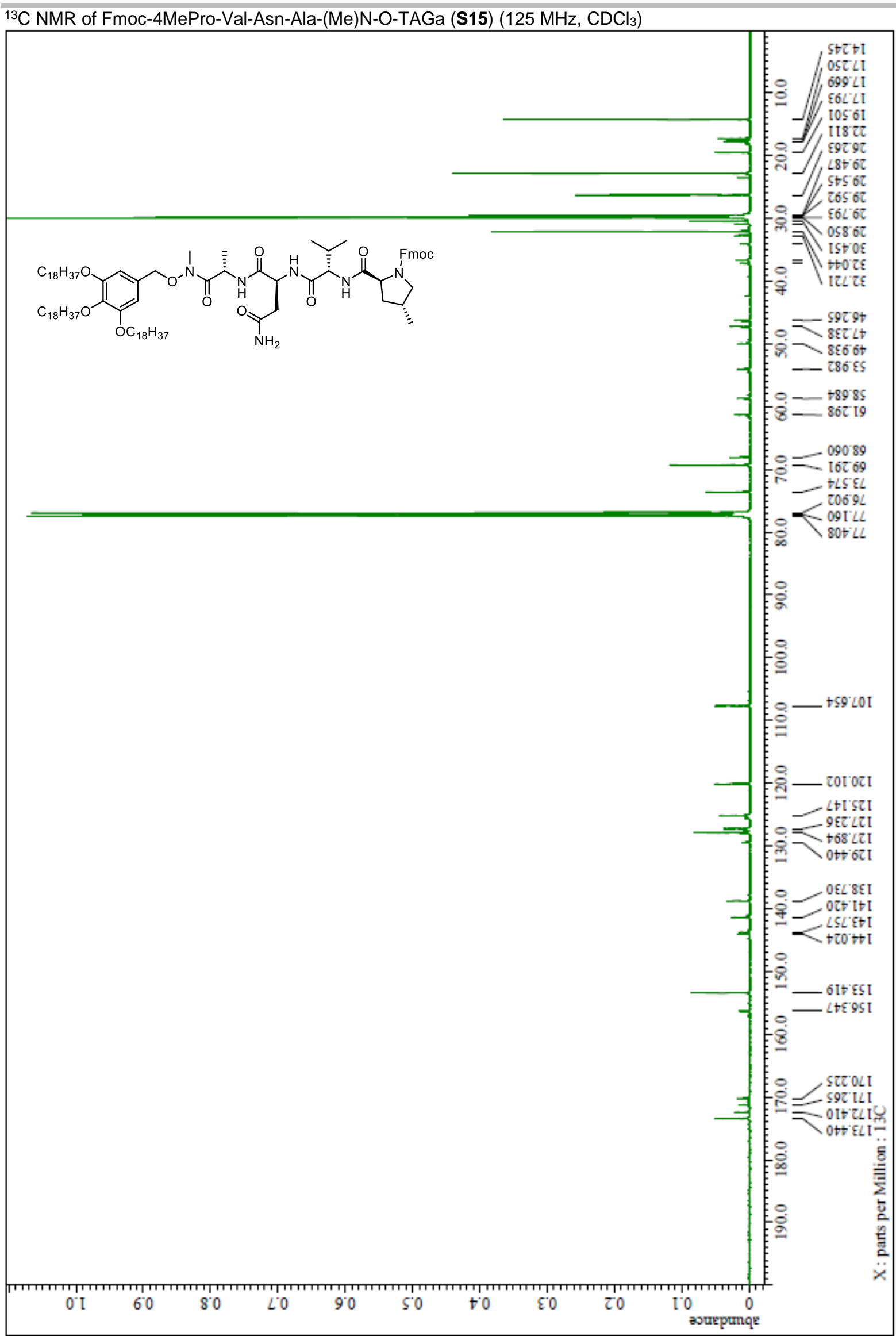




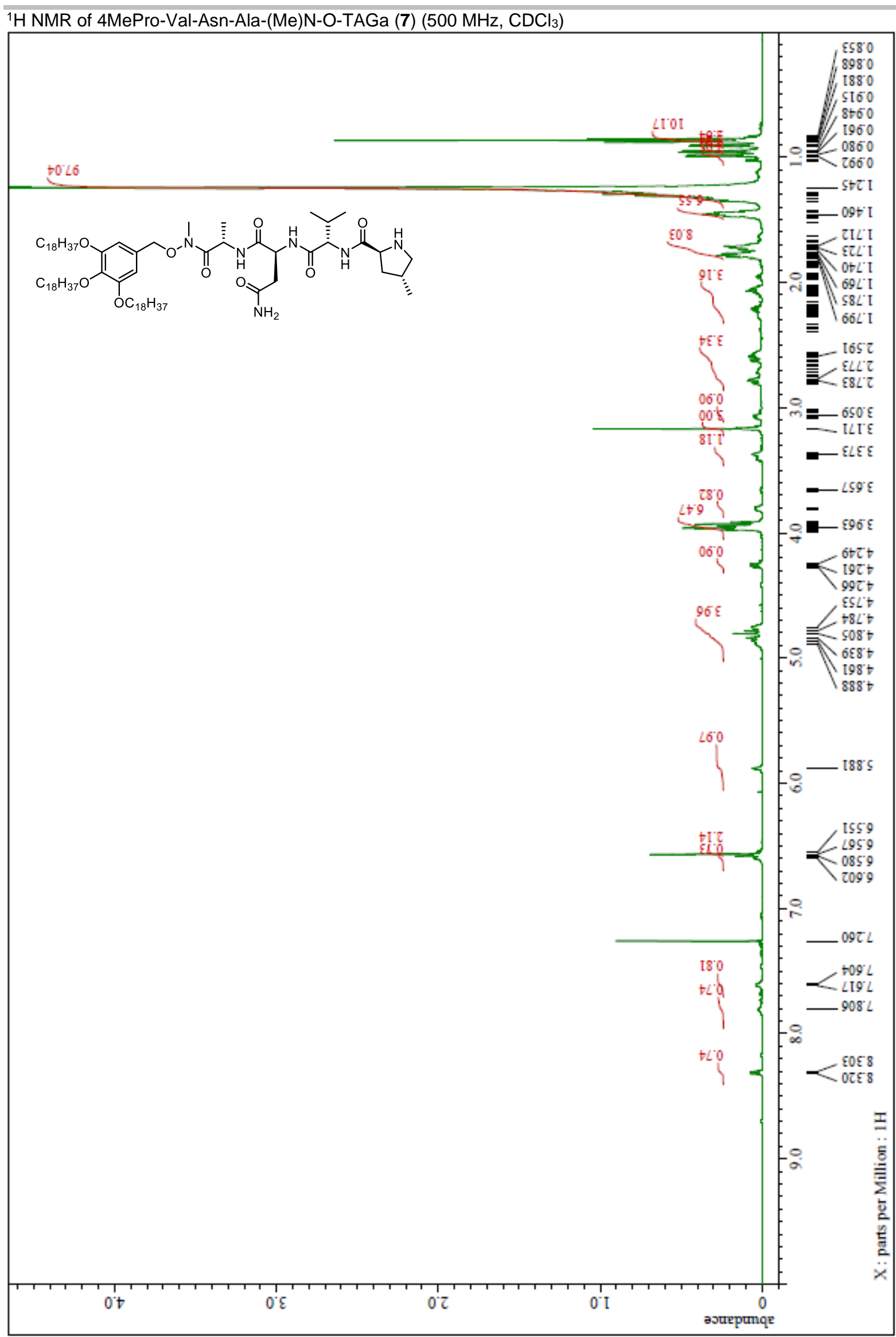




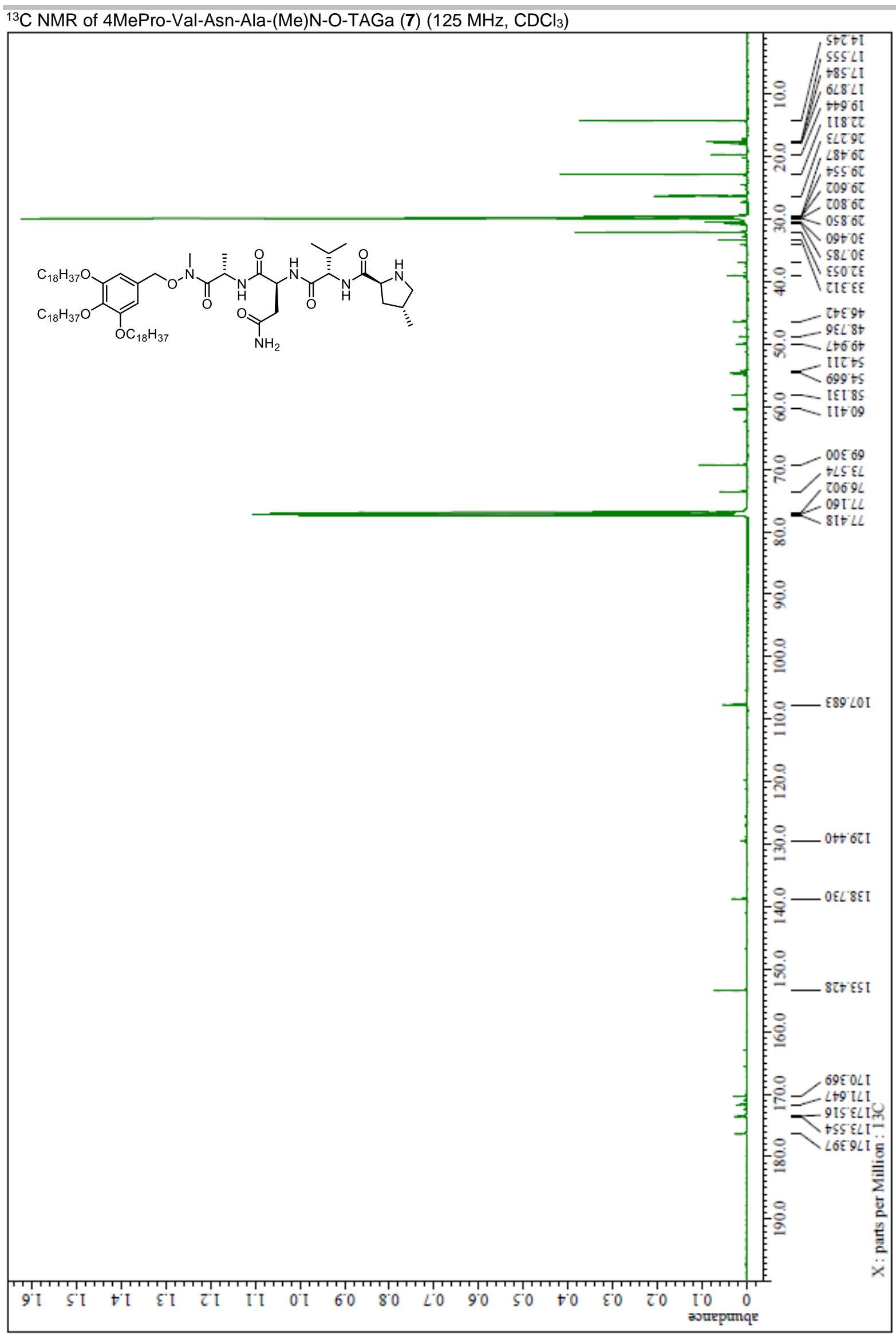




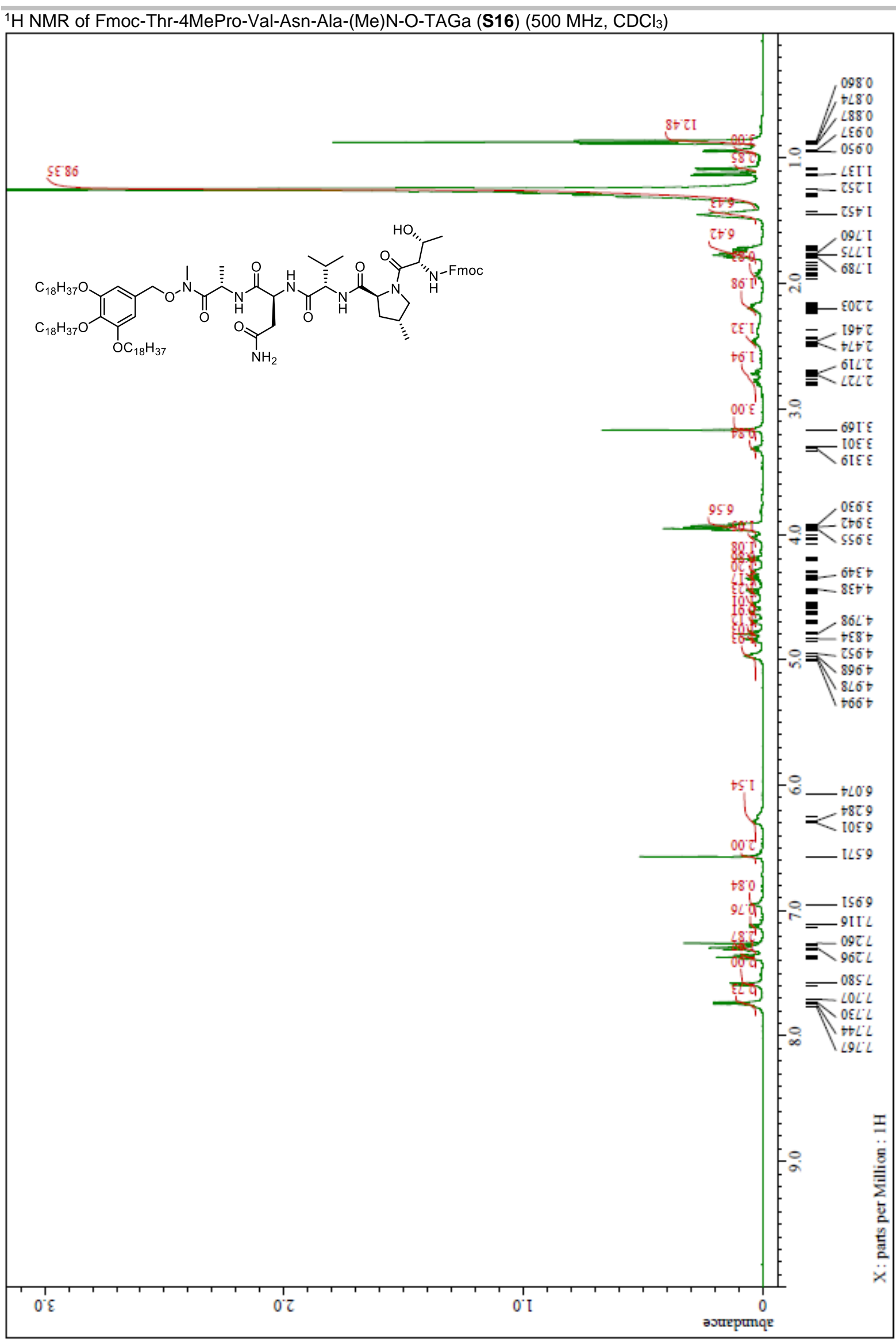




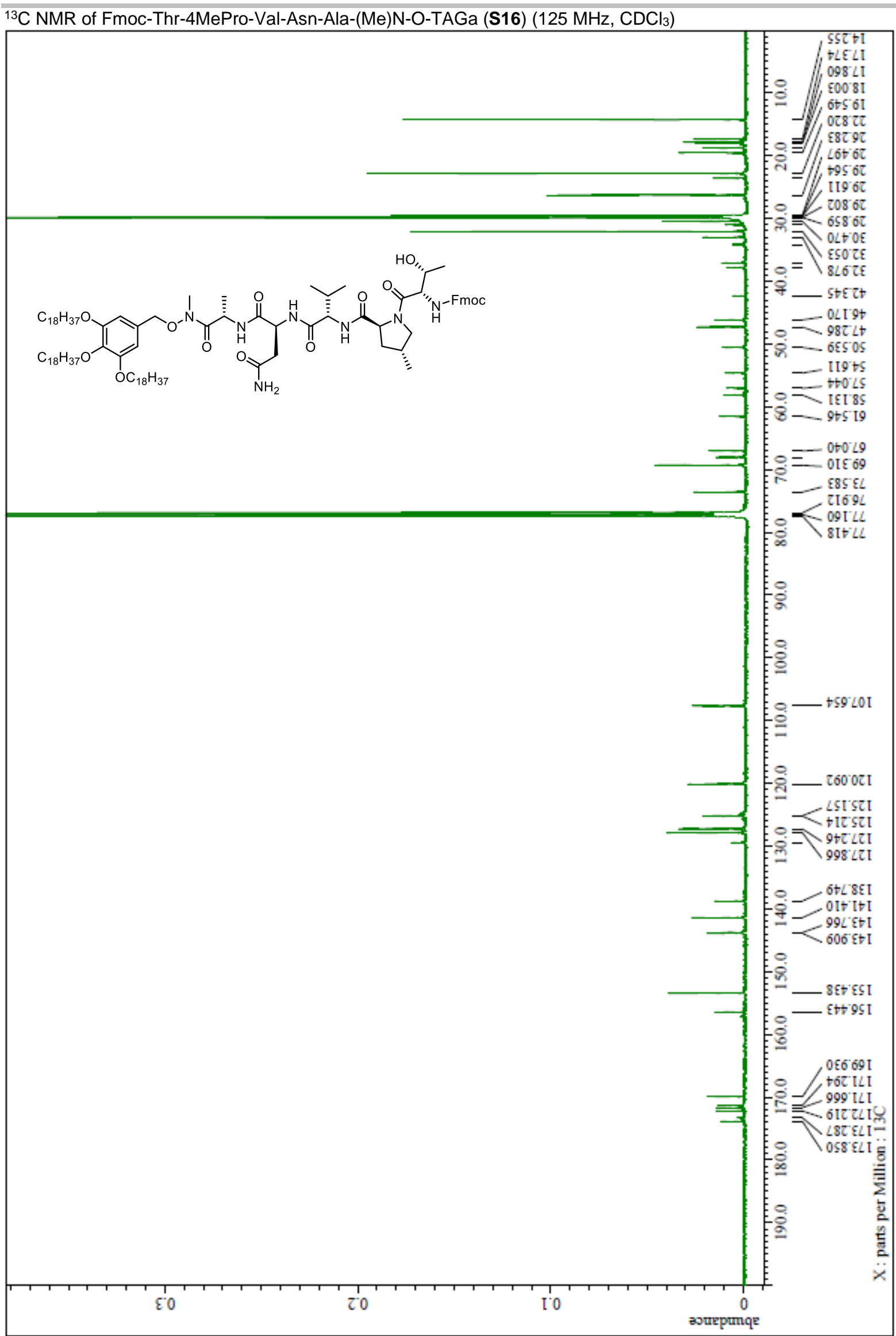




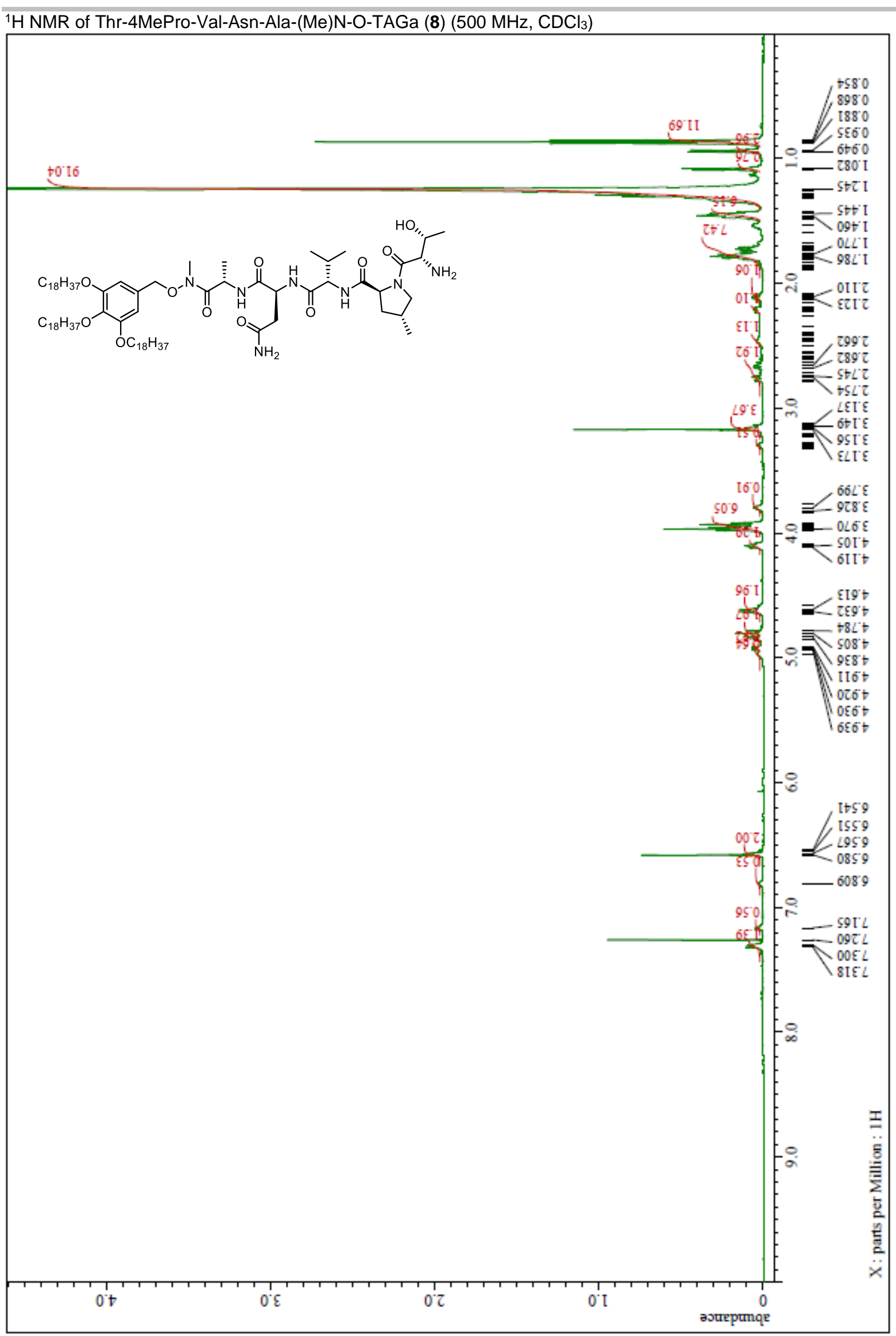




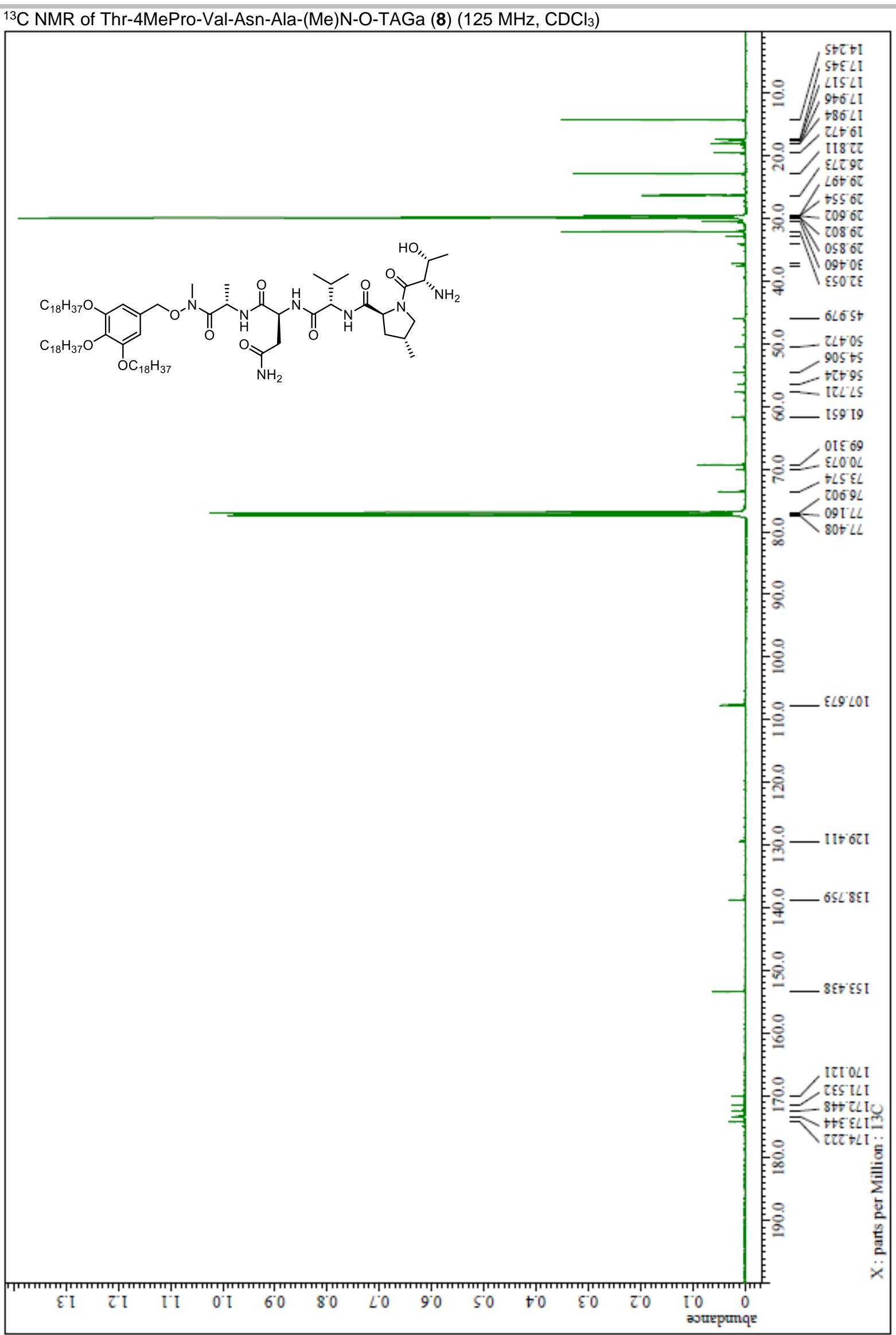




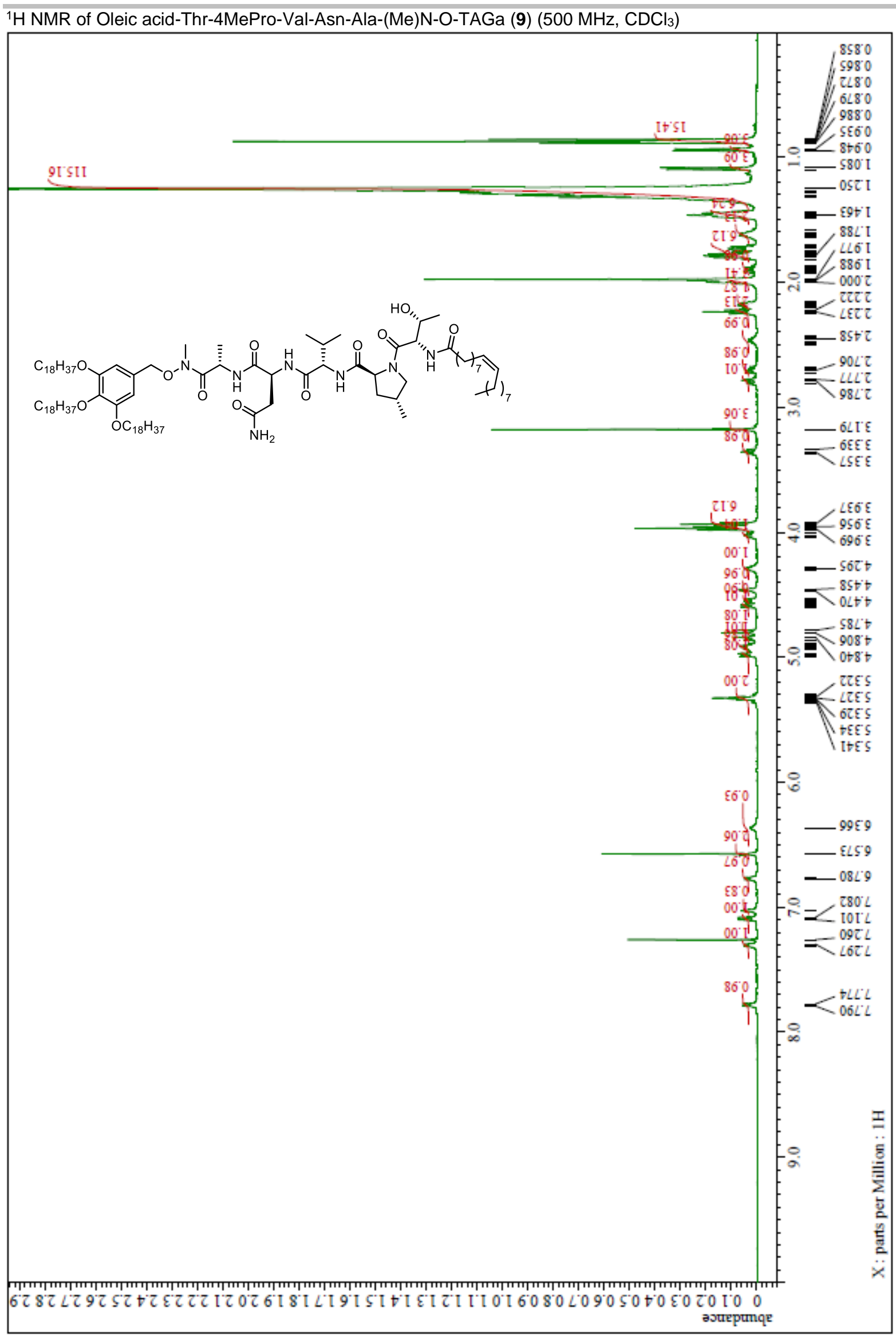




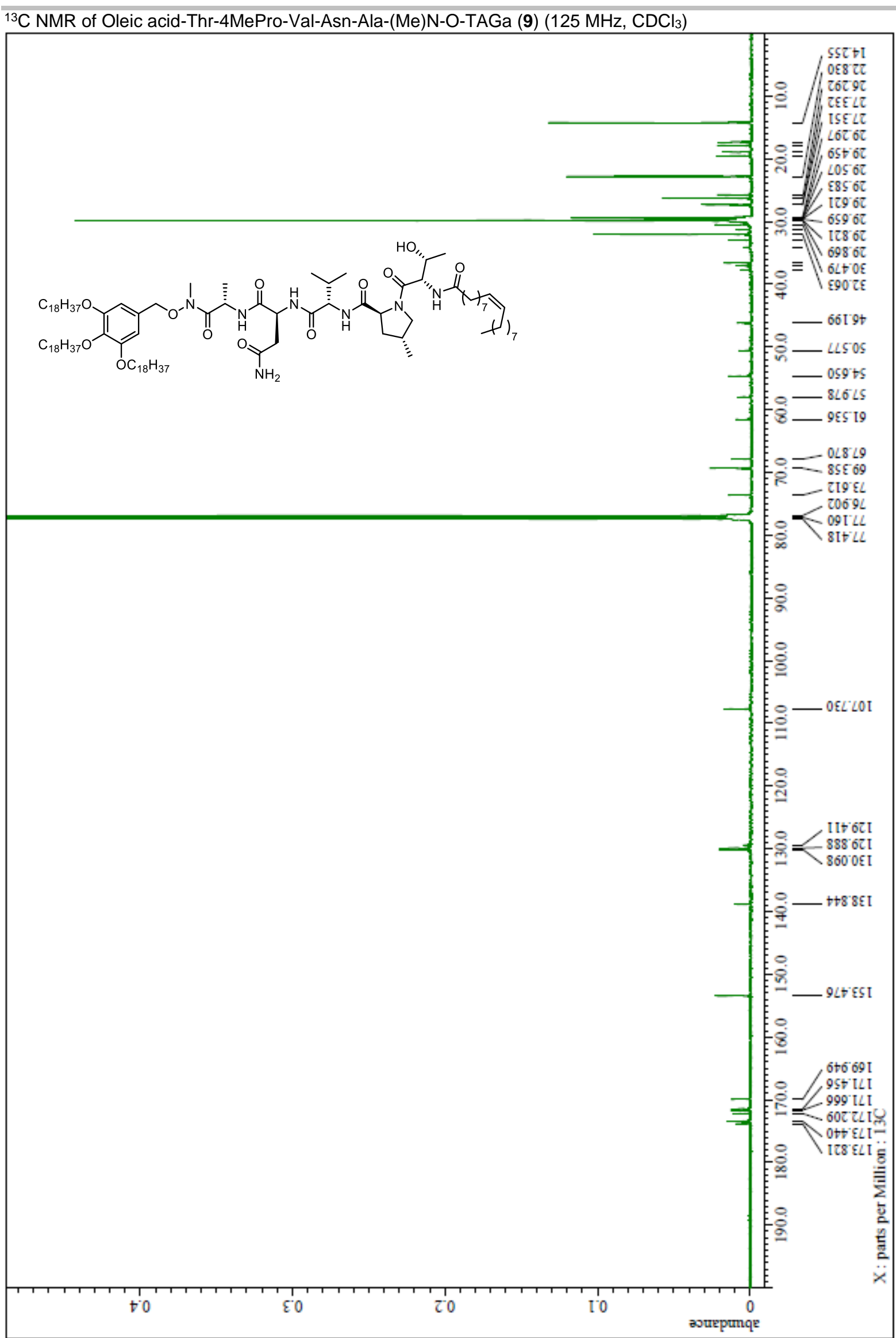




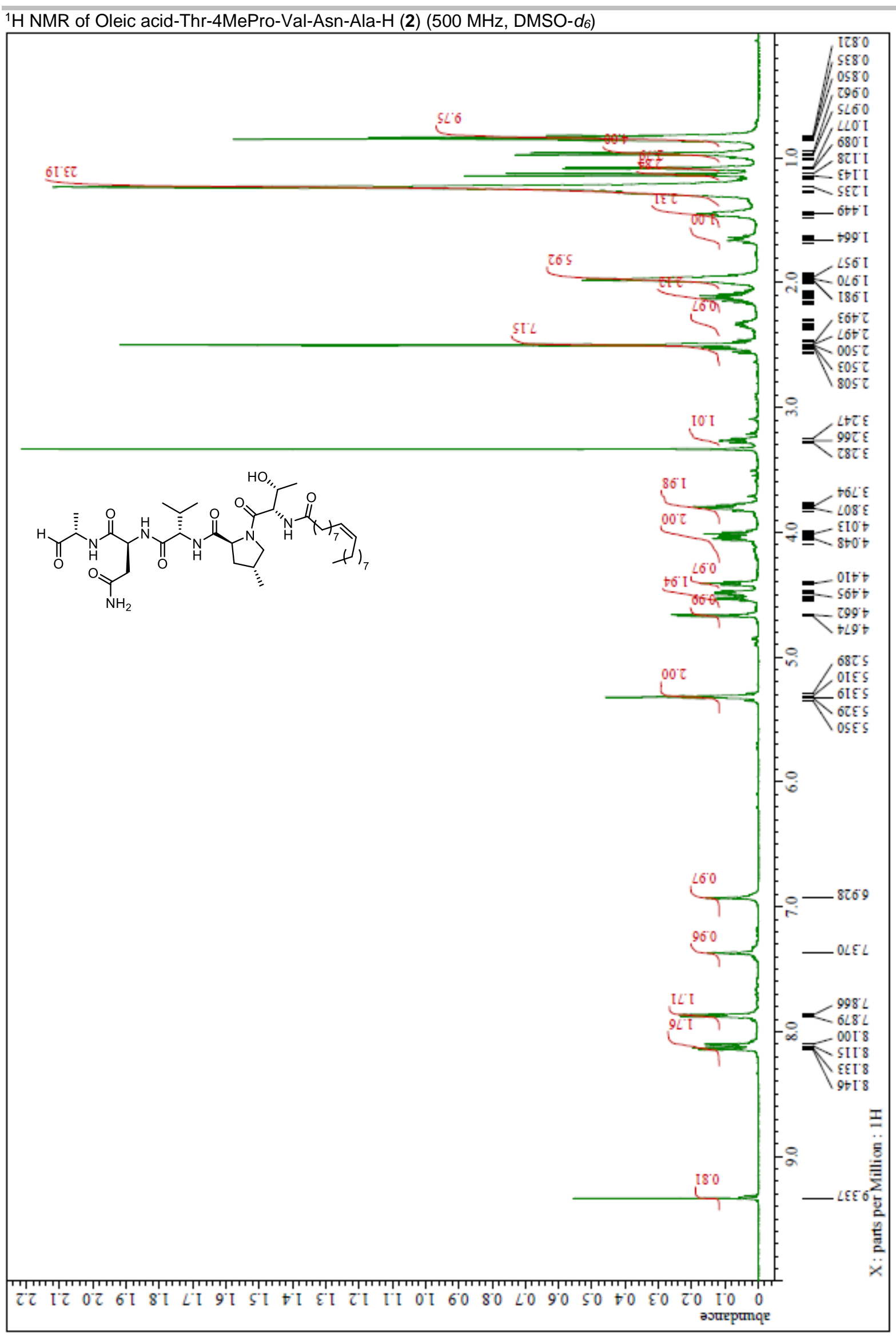


${ }^{13} \mathrm{C}$ NMR of Oleic acid-Thr-4MePro-Val-Asn-Ala-H (2) (125 MHz, DMSO-d6)

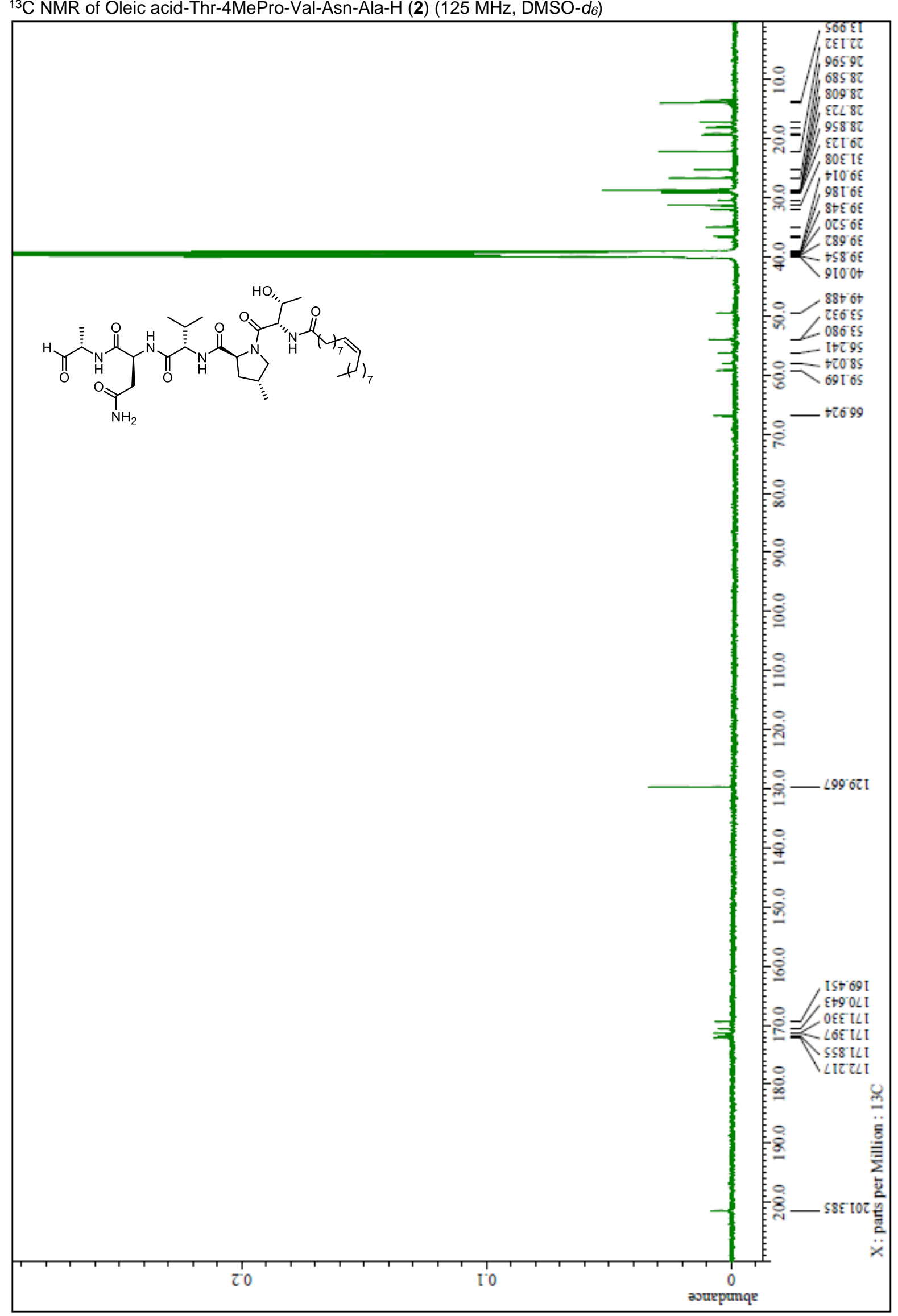

62 


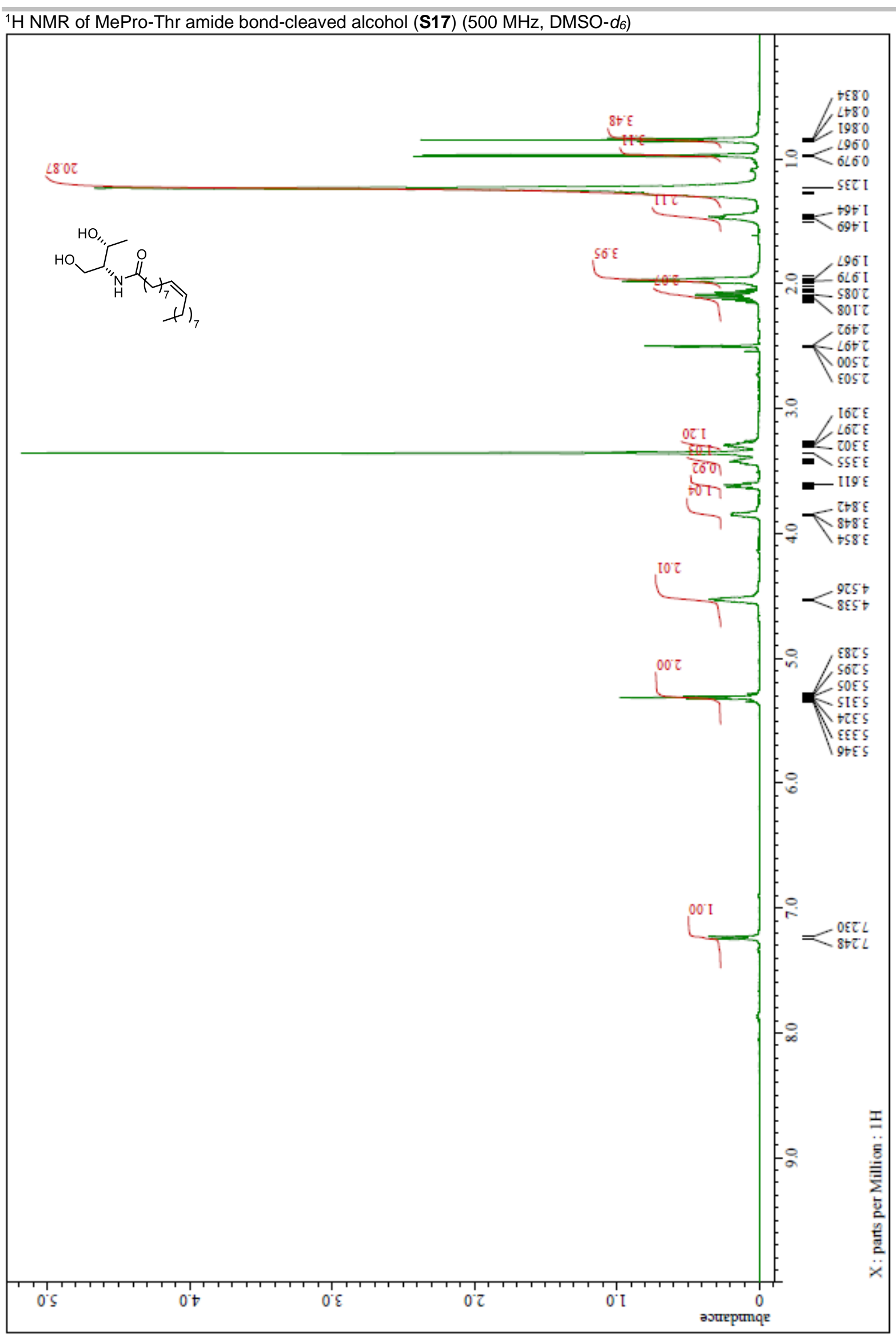




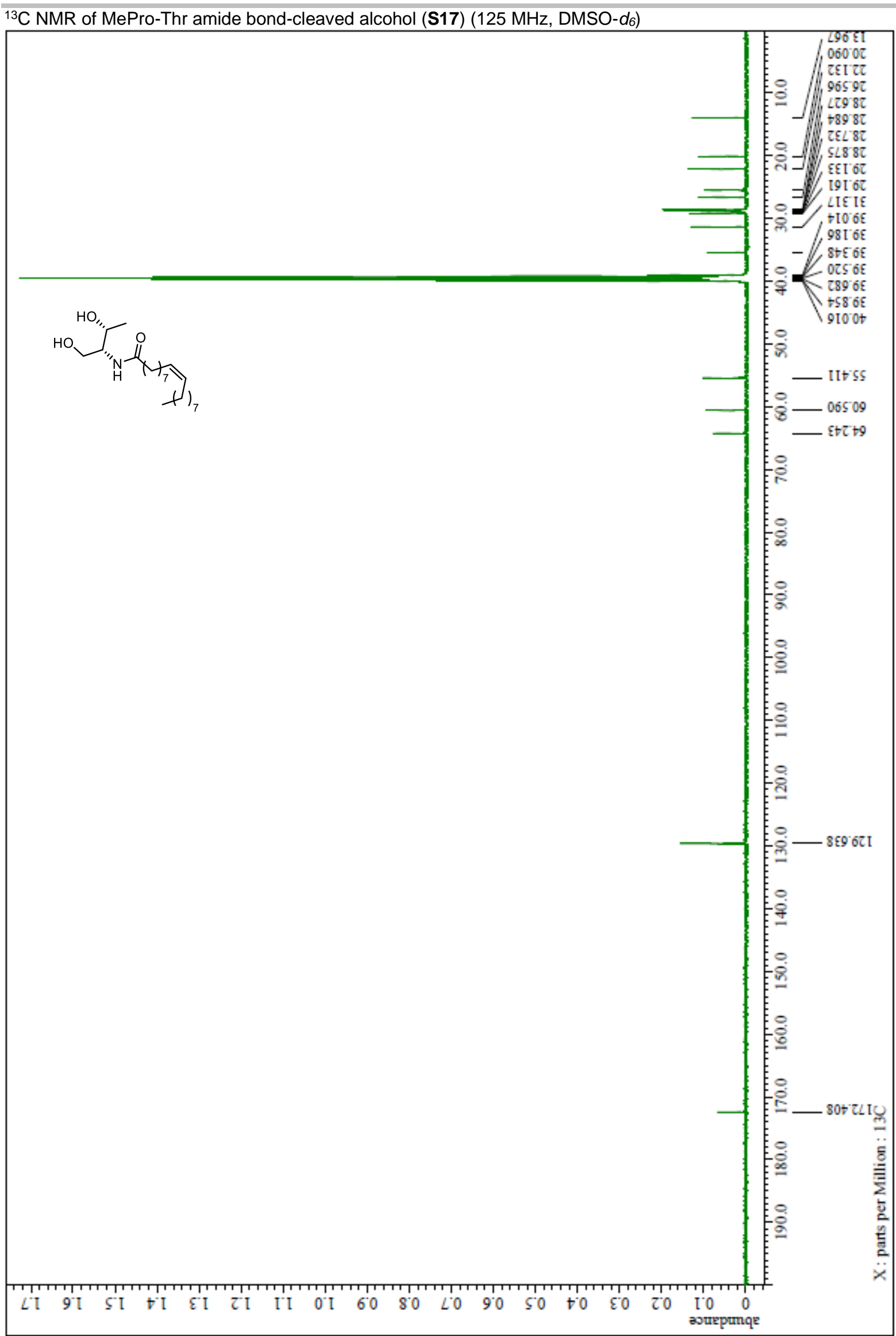




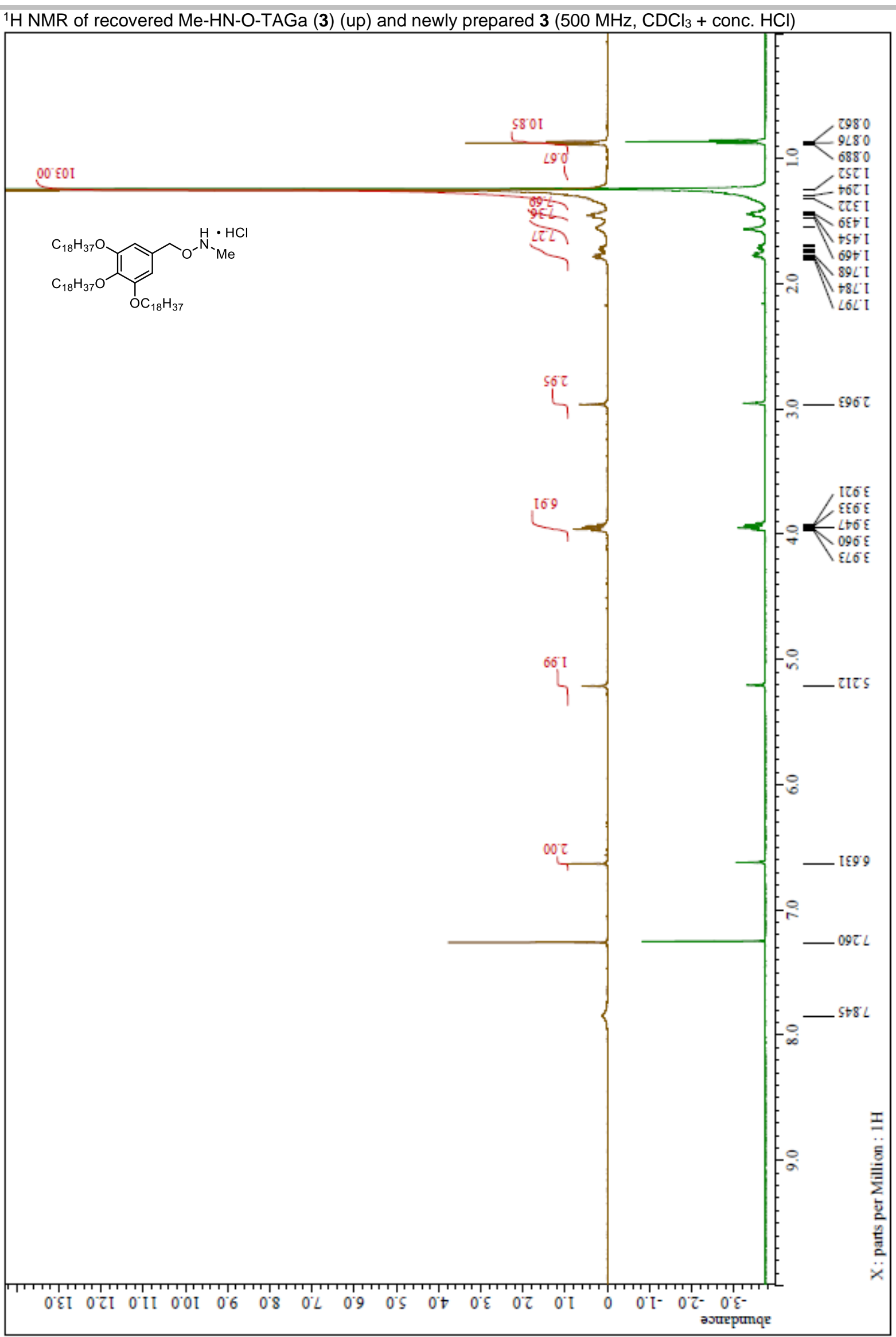




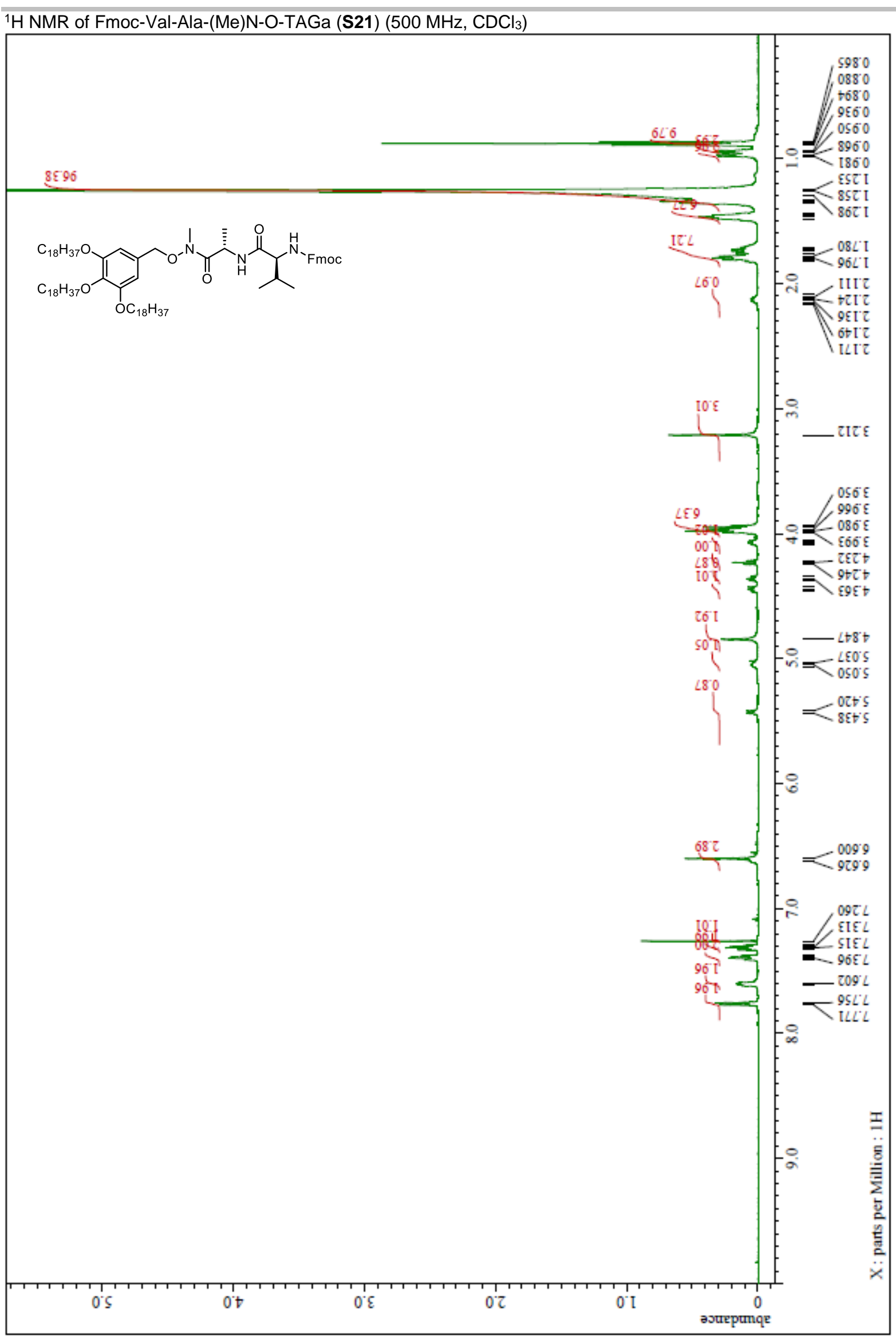




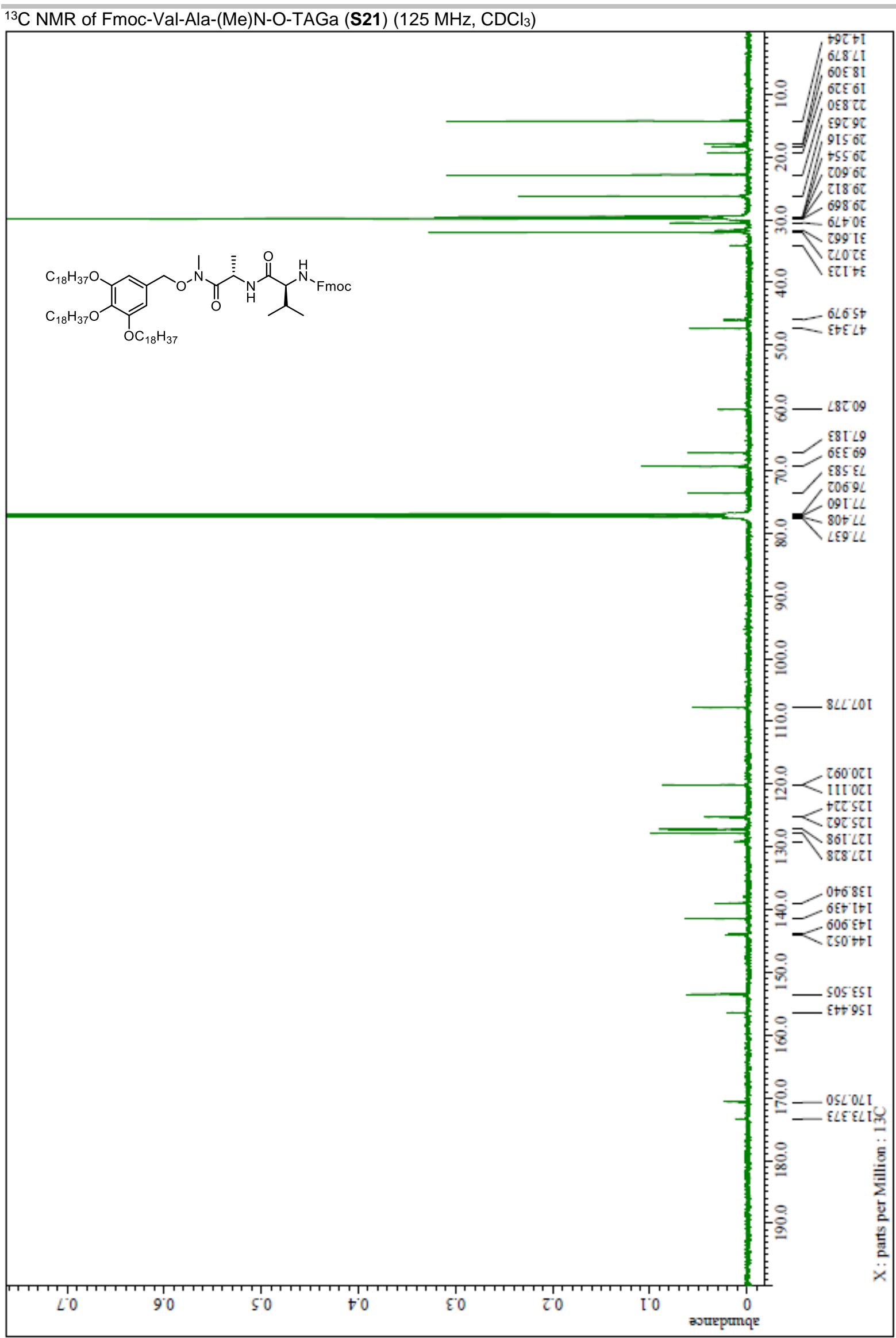




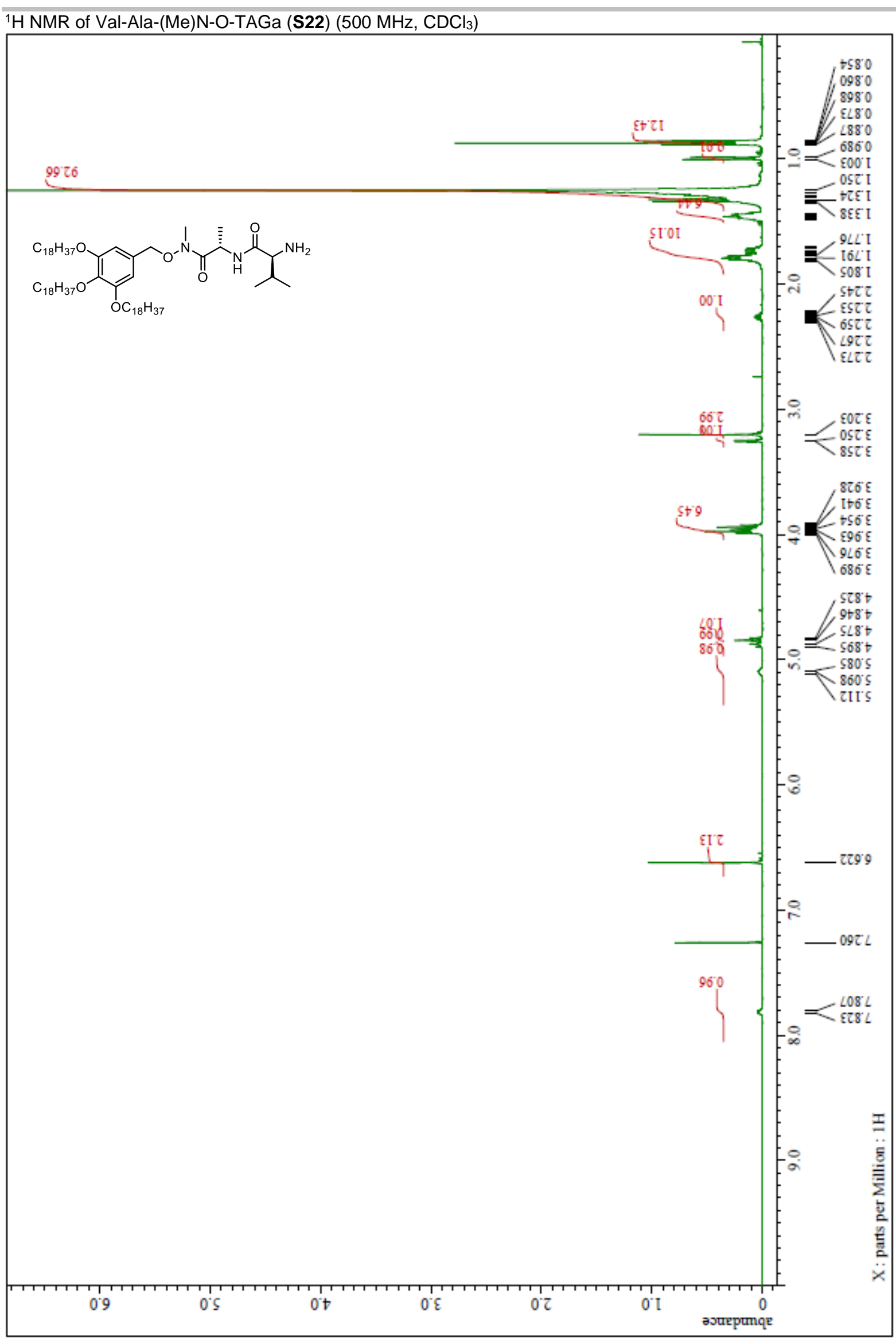




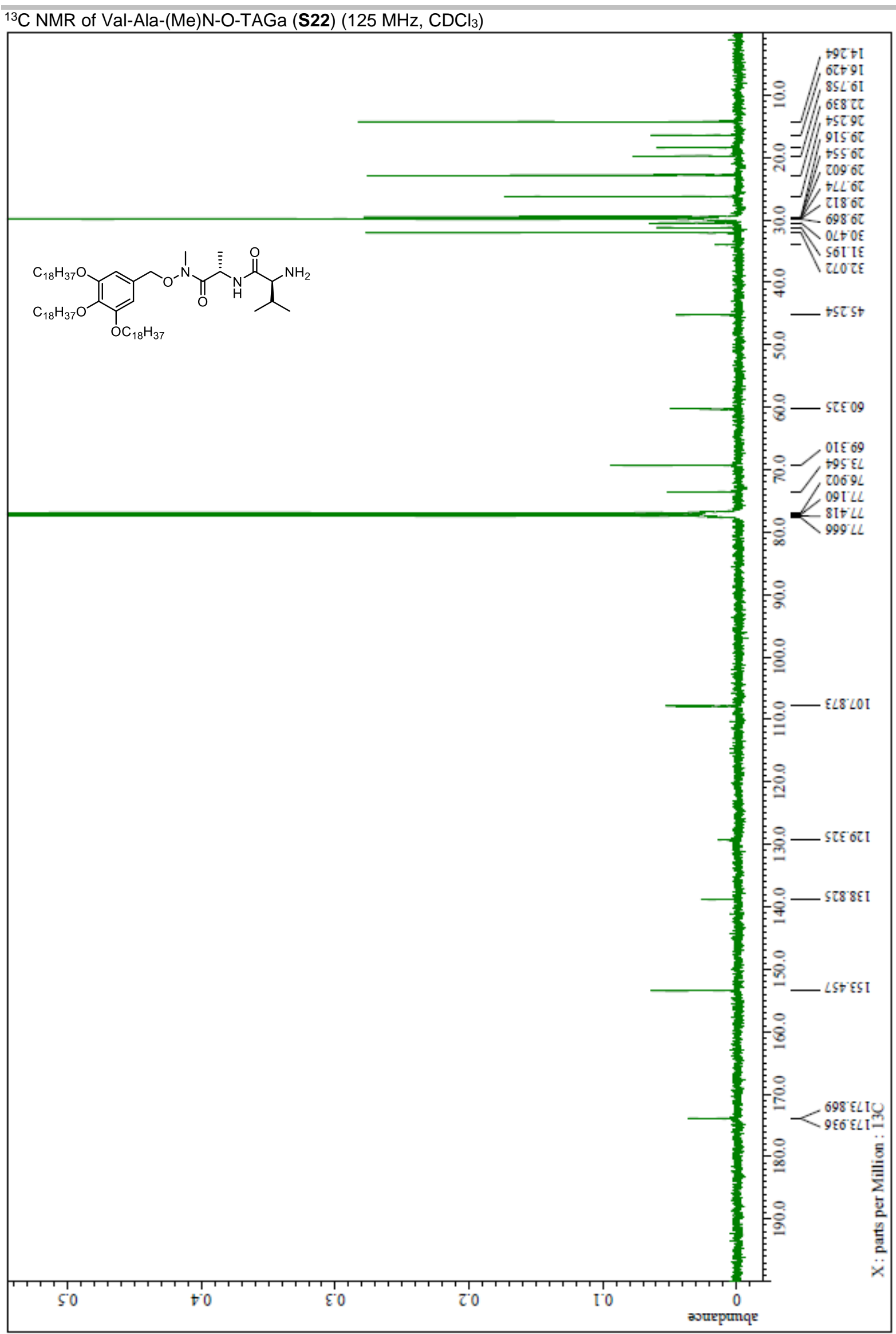




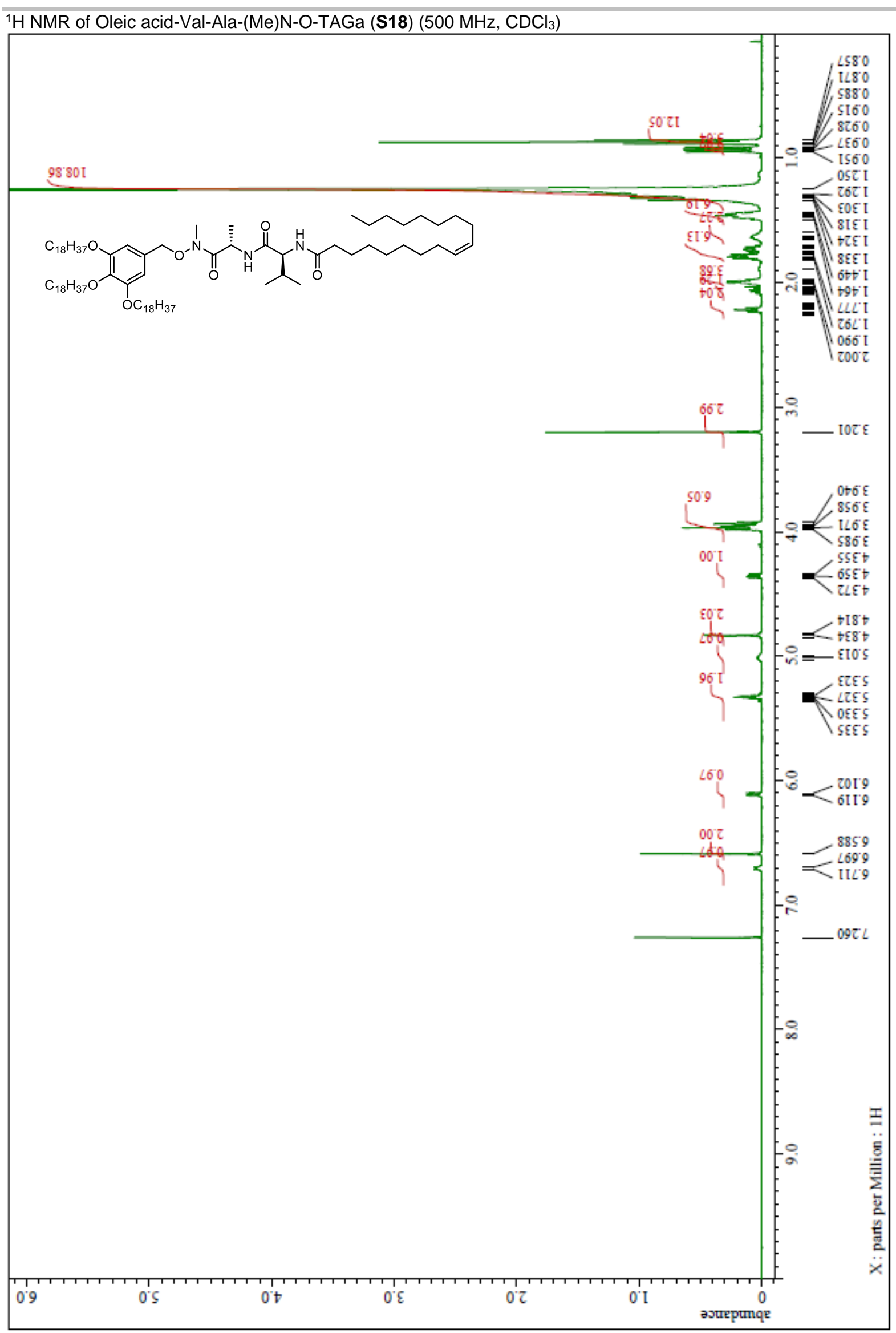




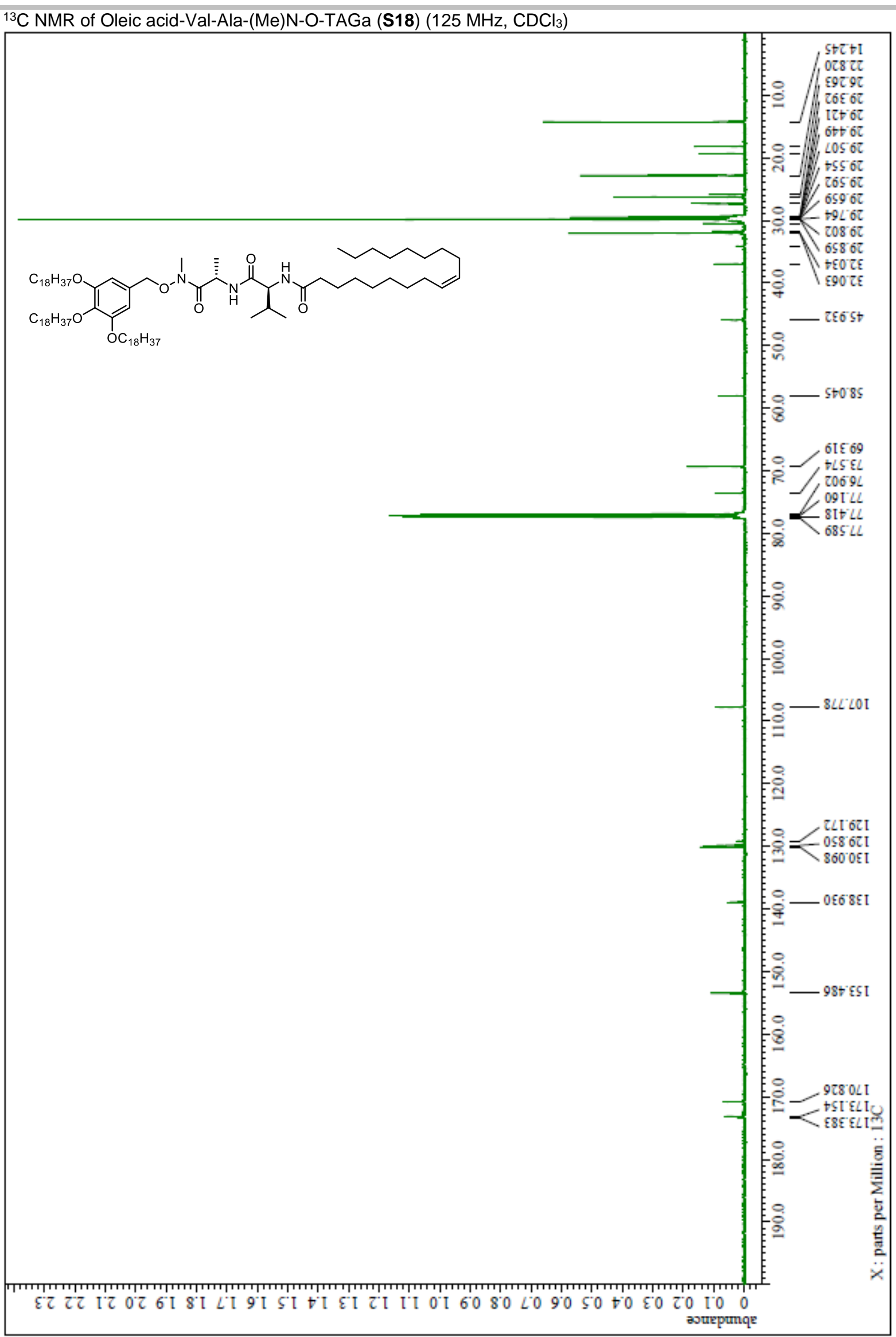




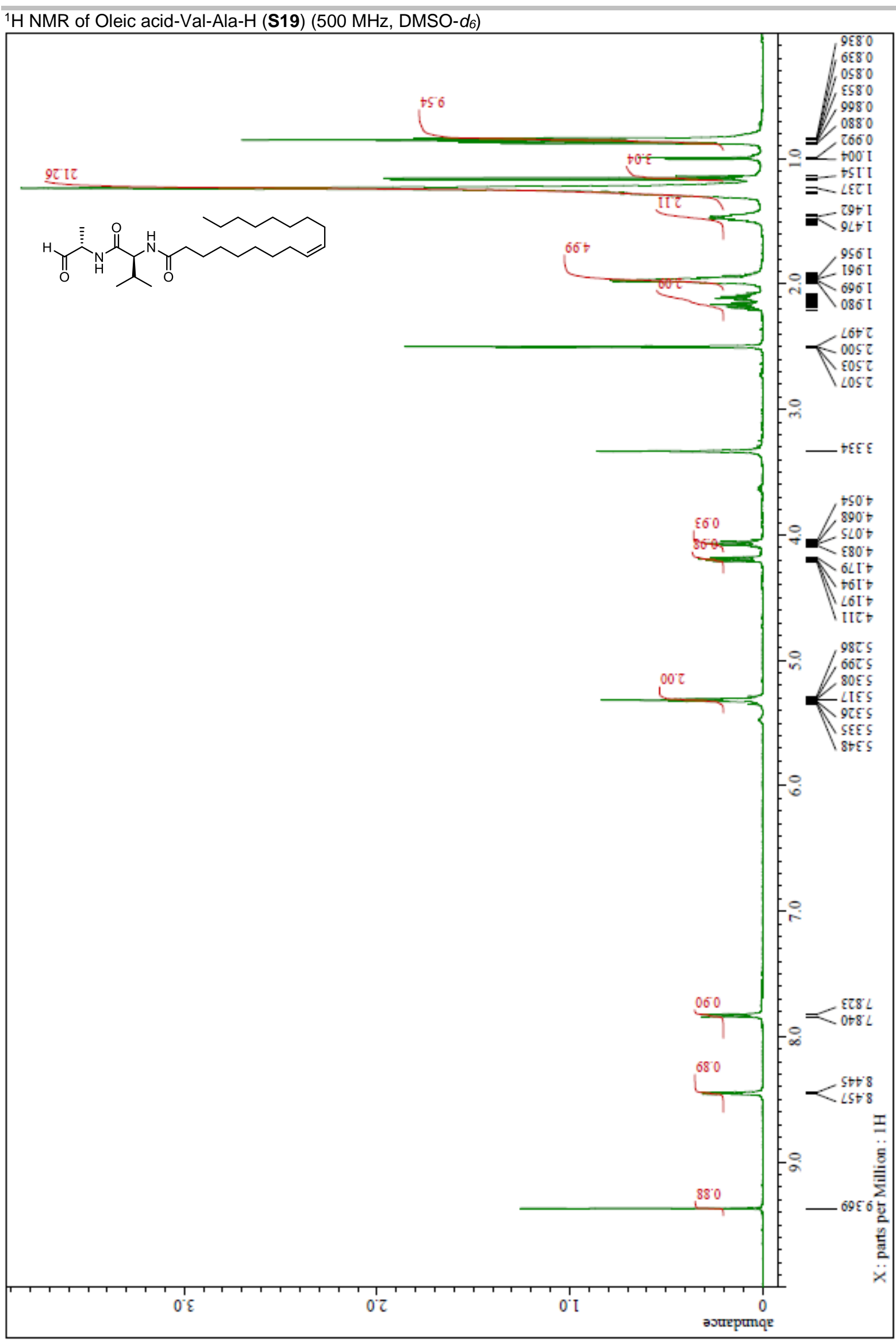




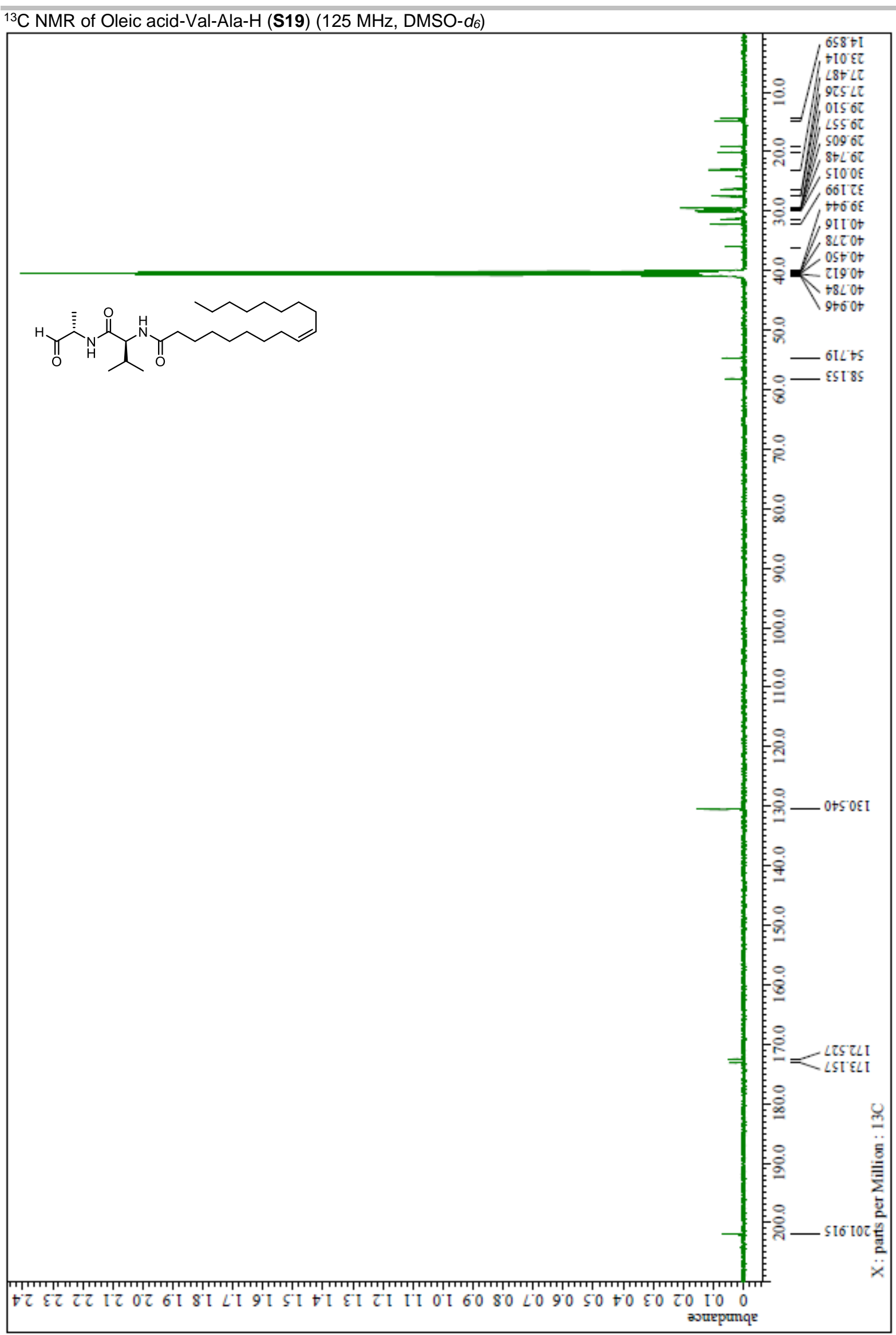




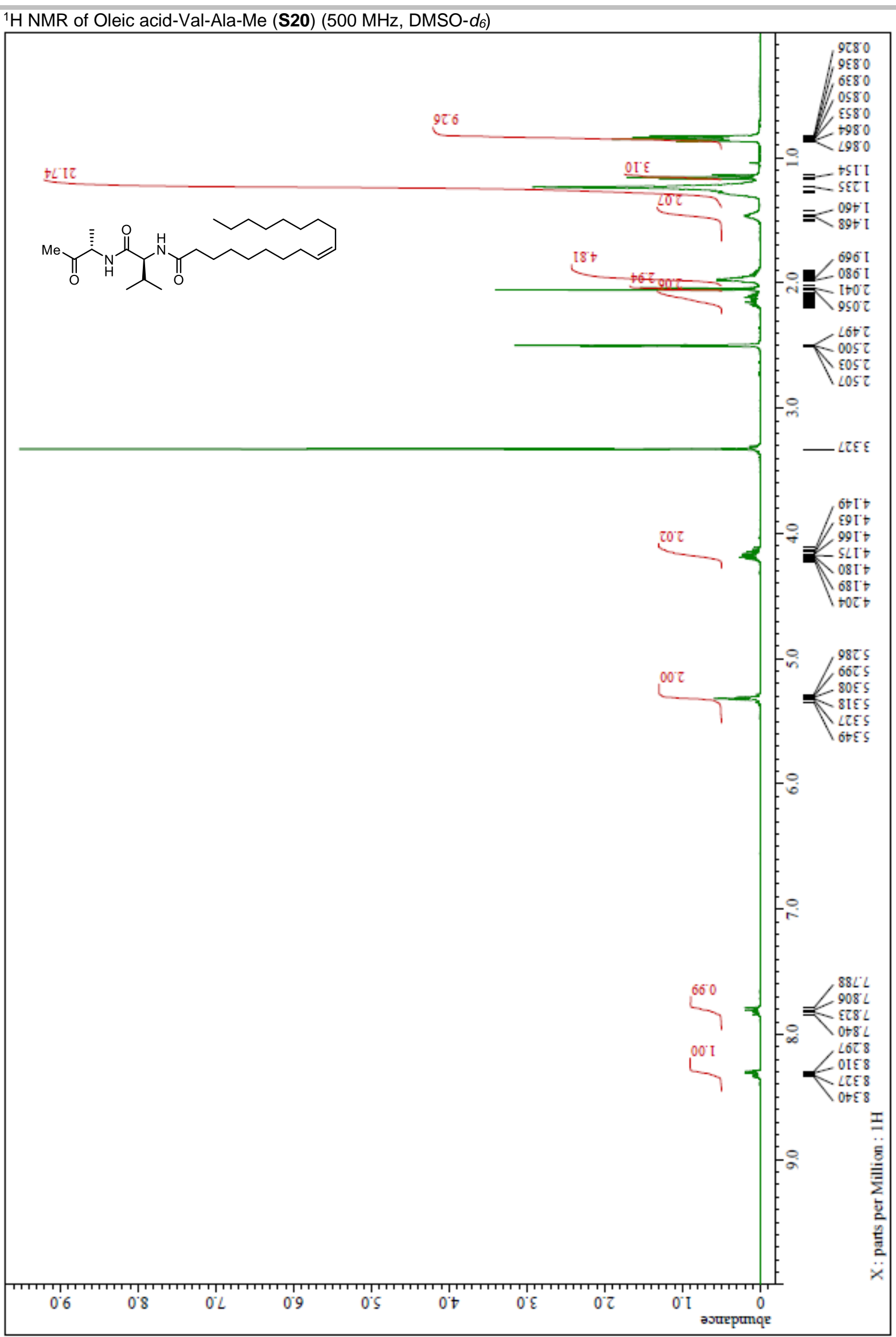




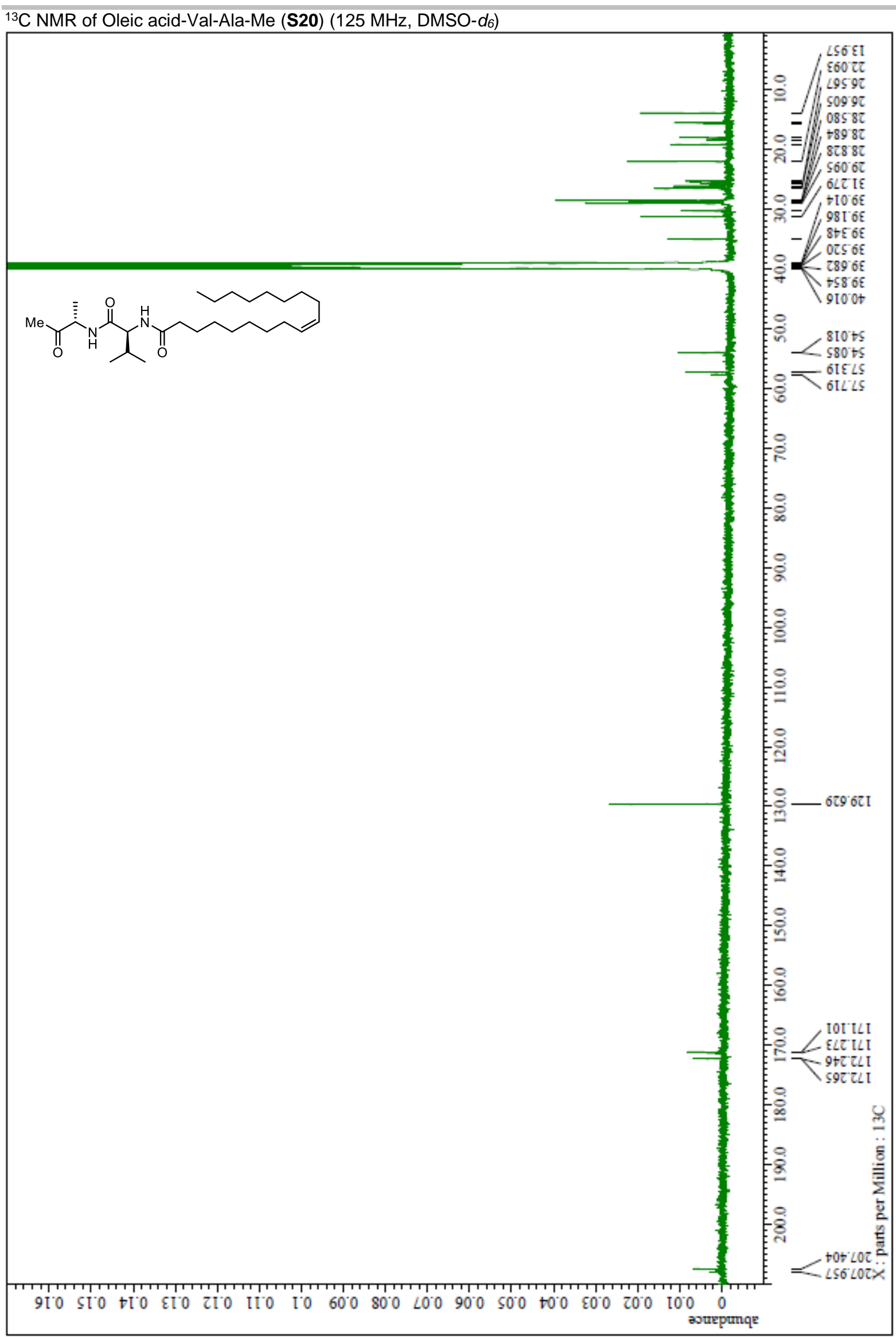




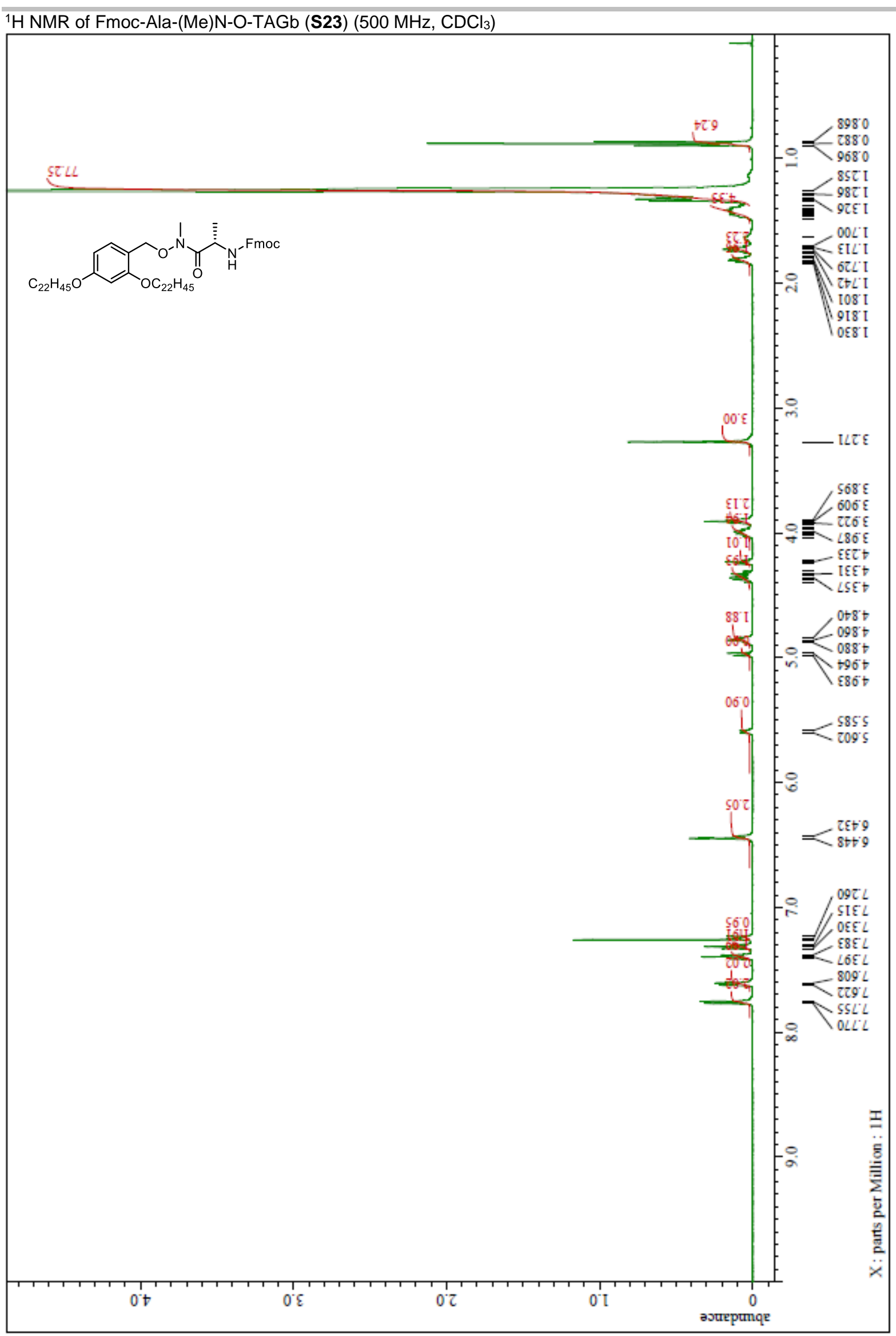




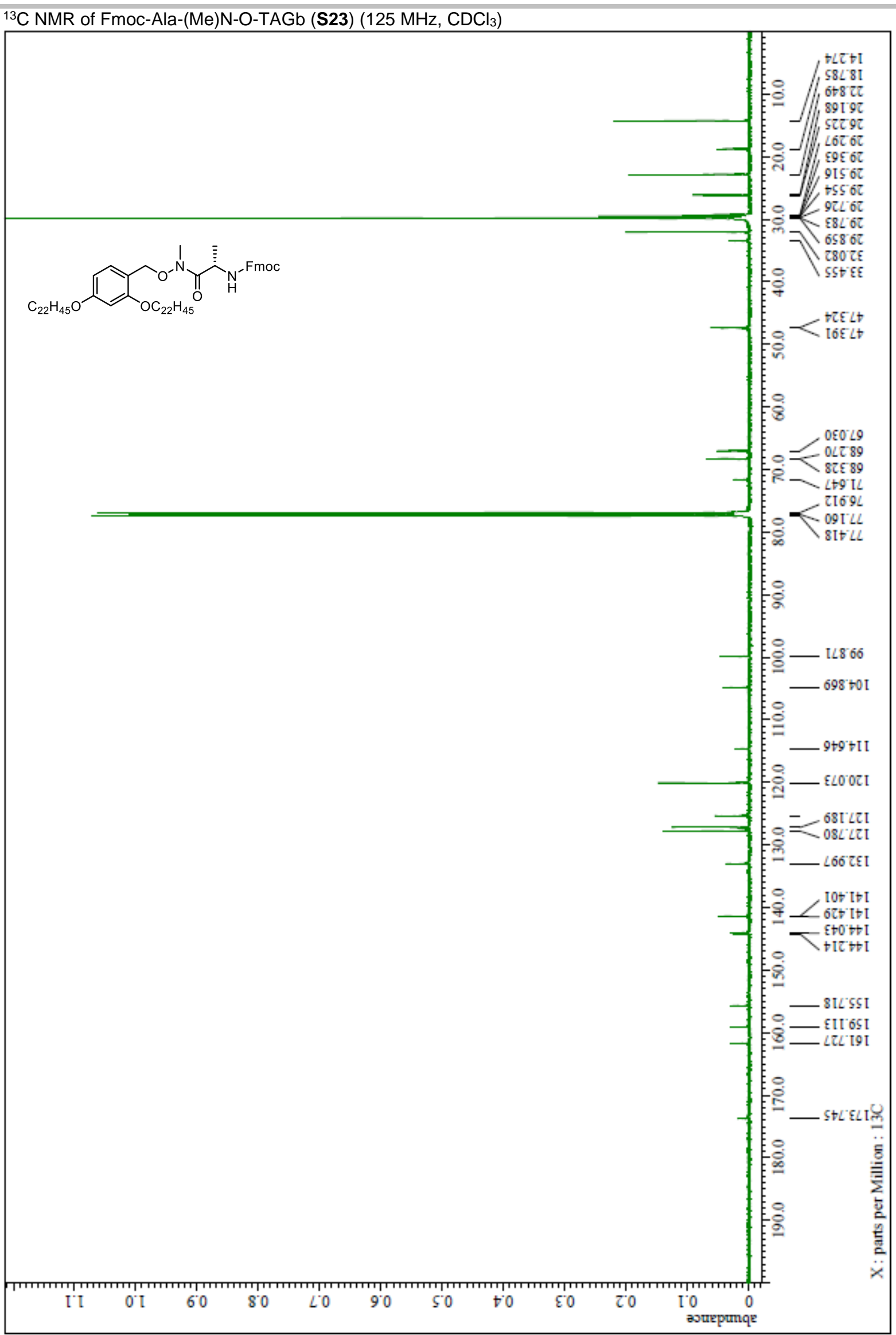




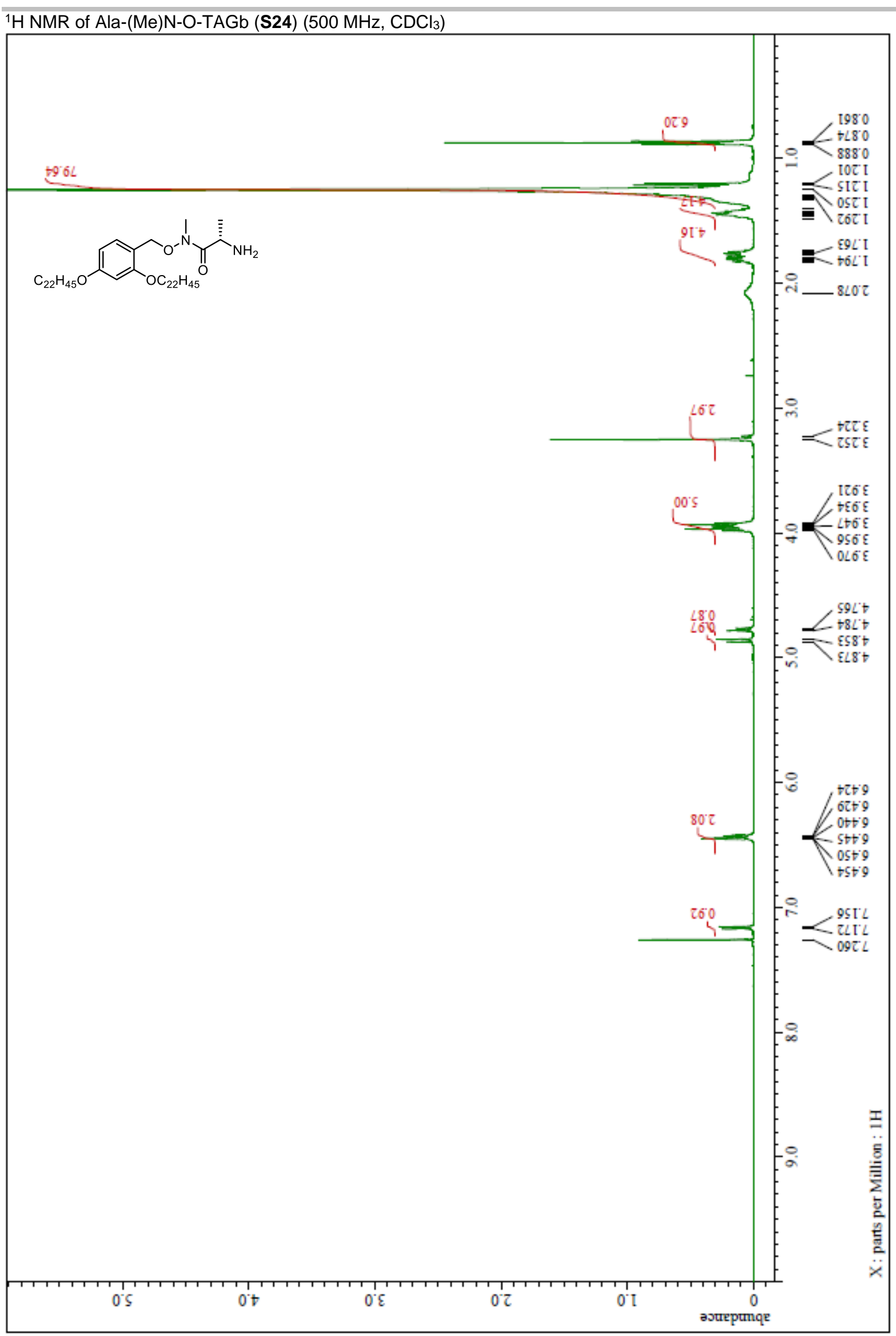




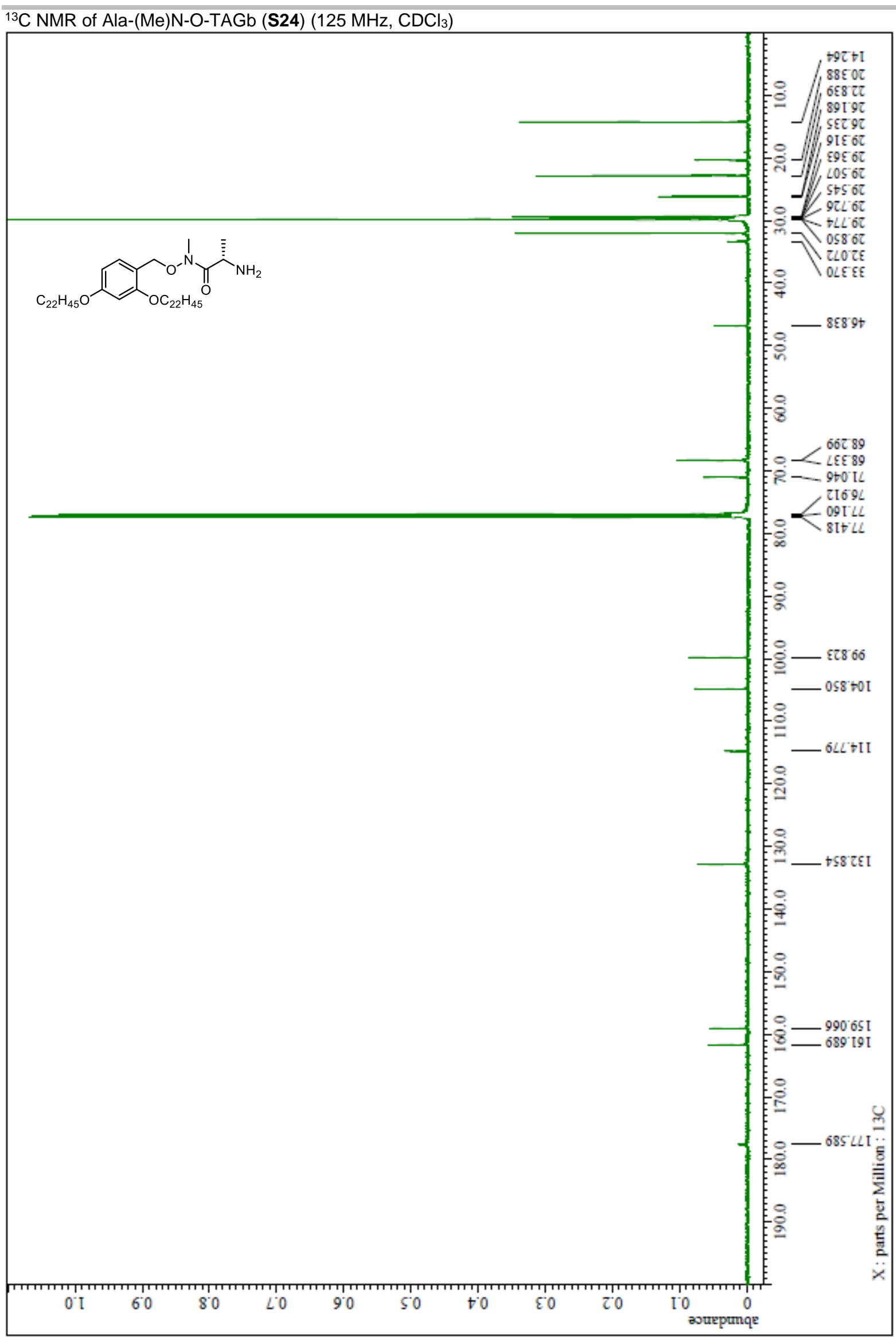




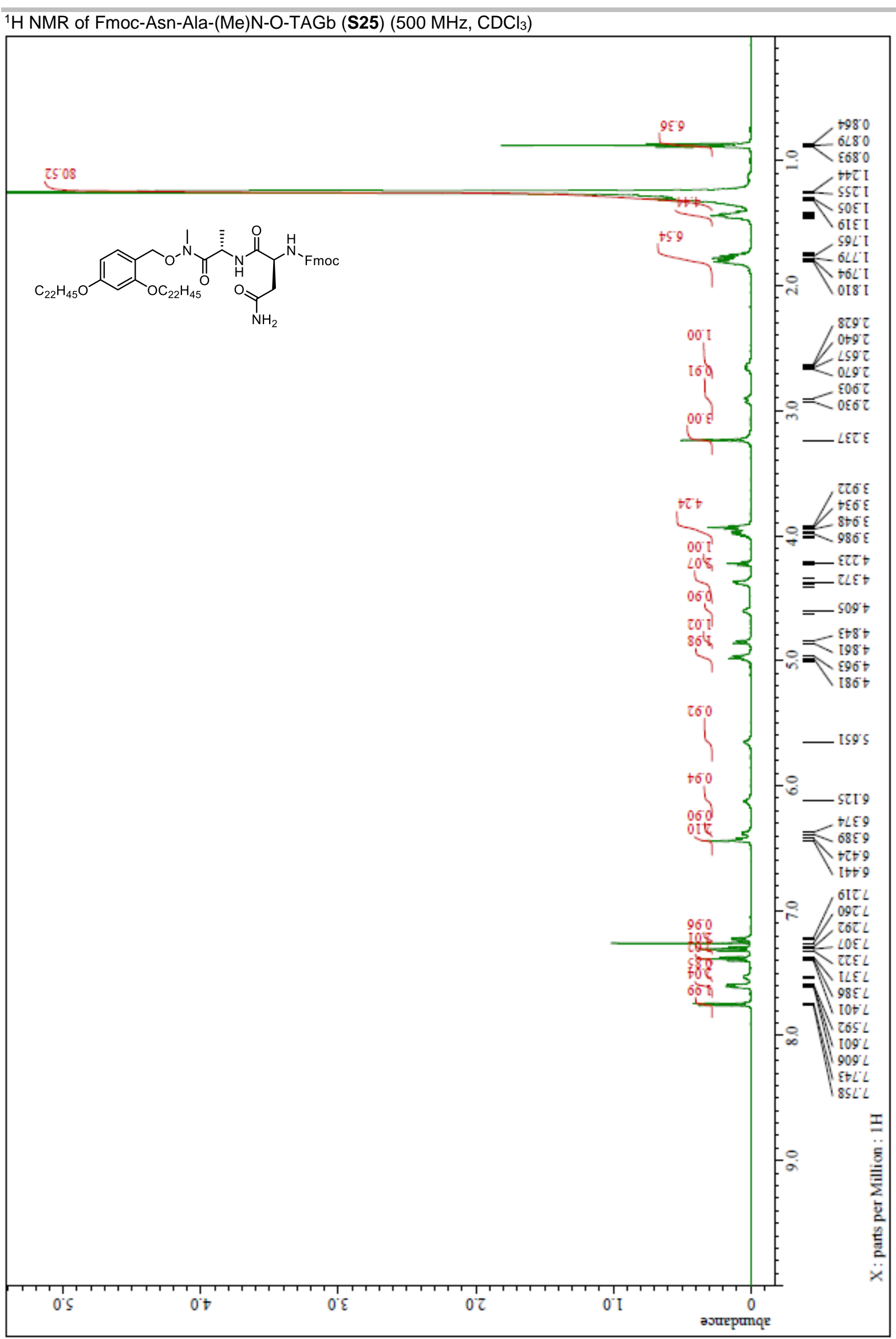




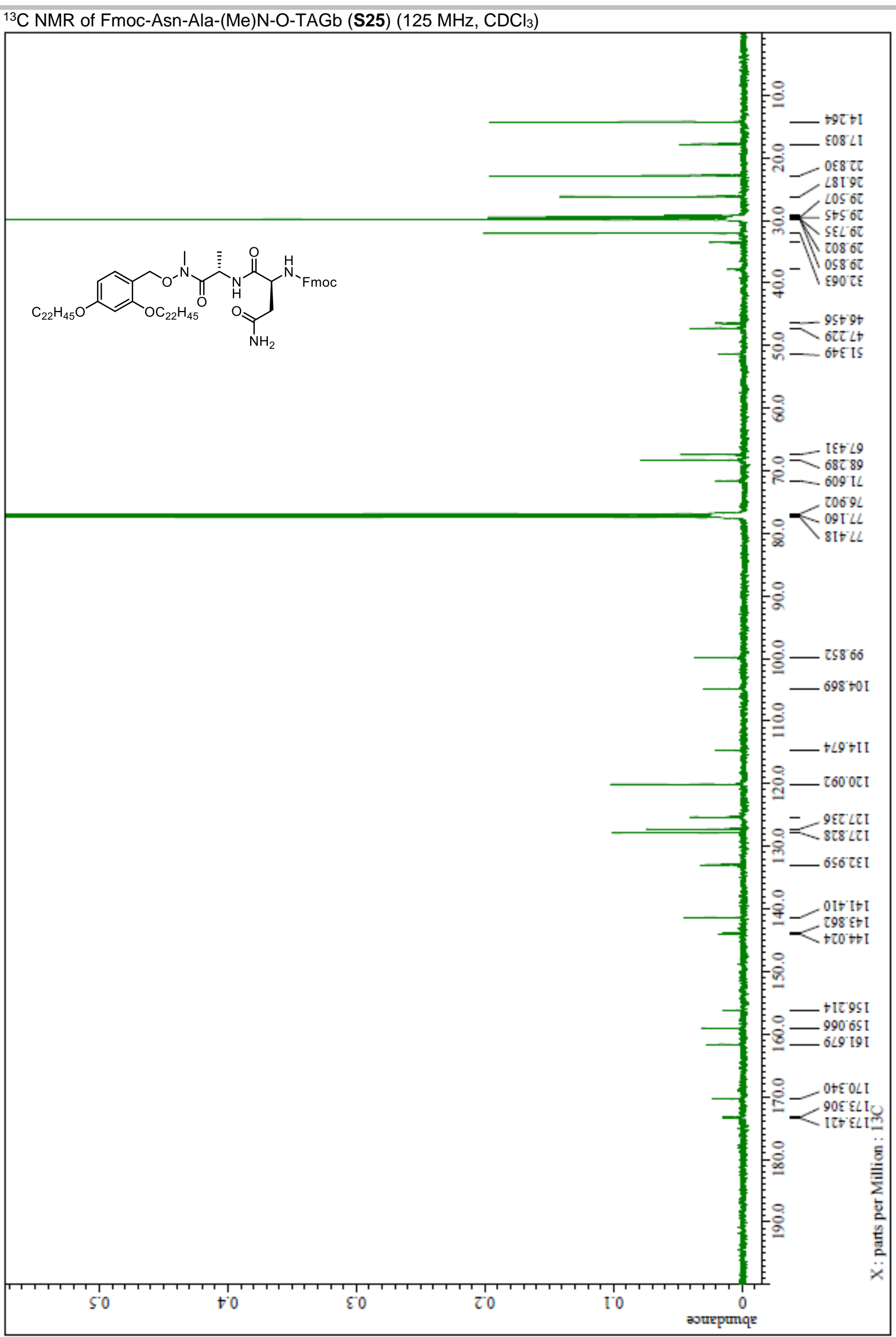




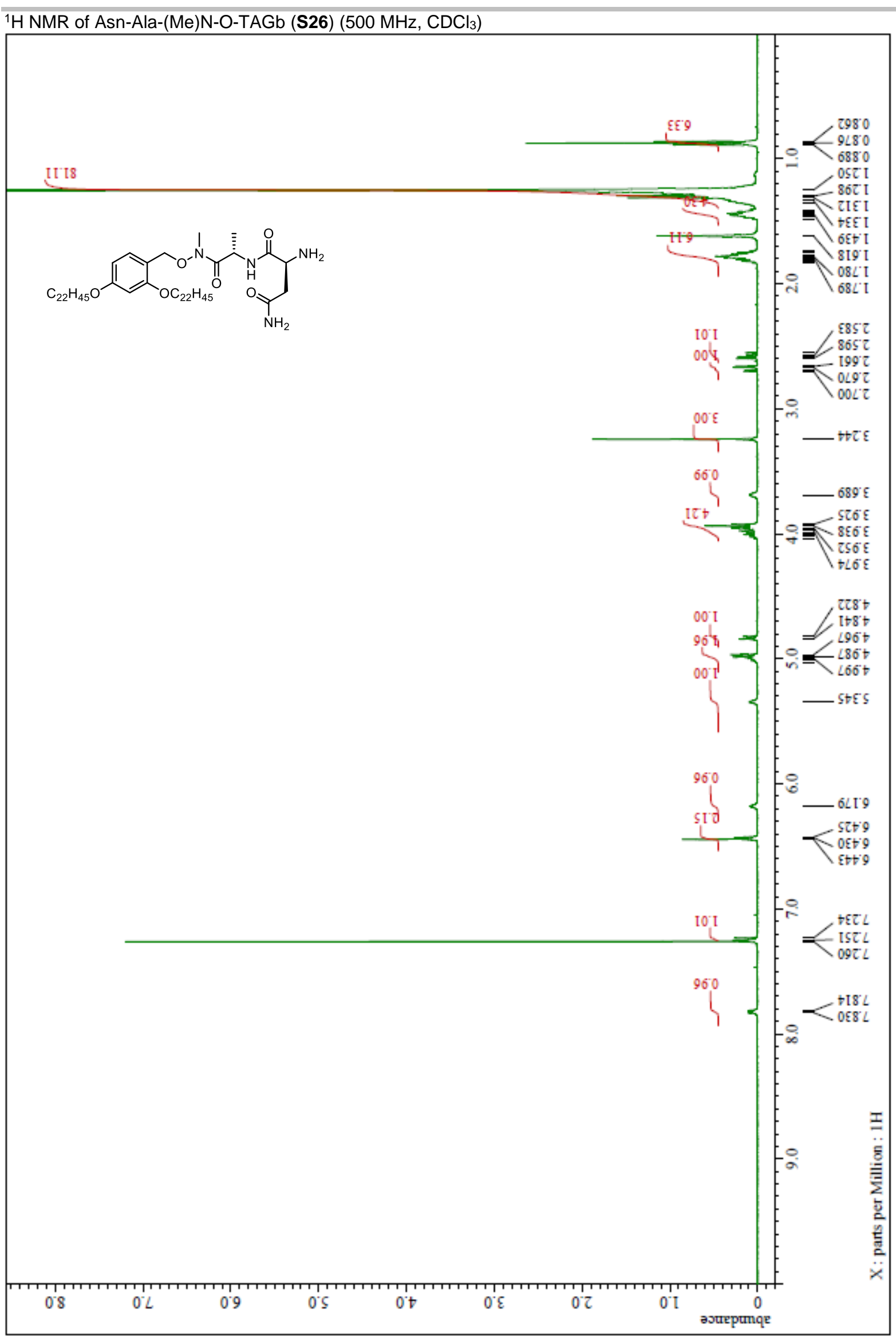




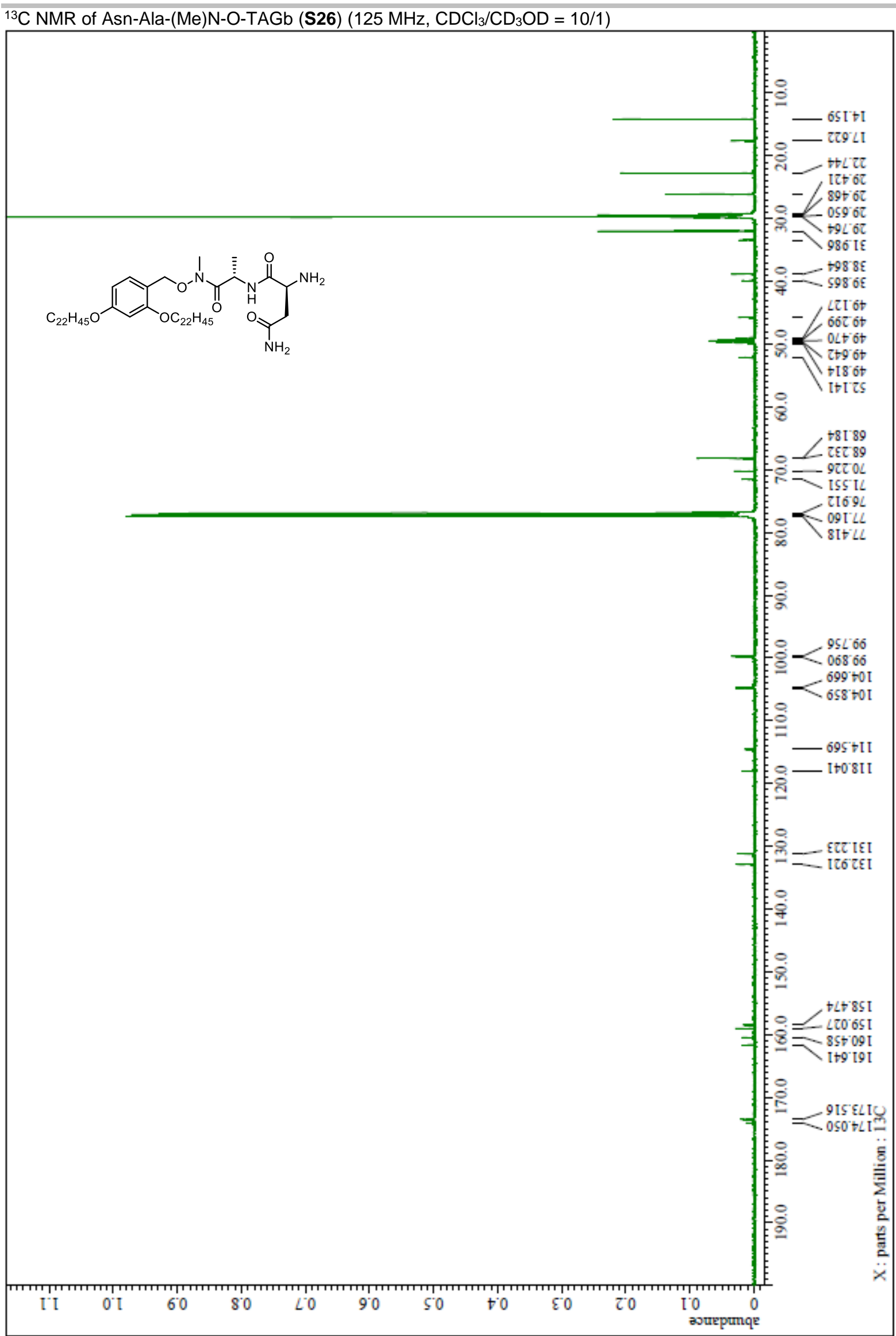




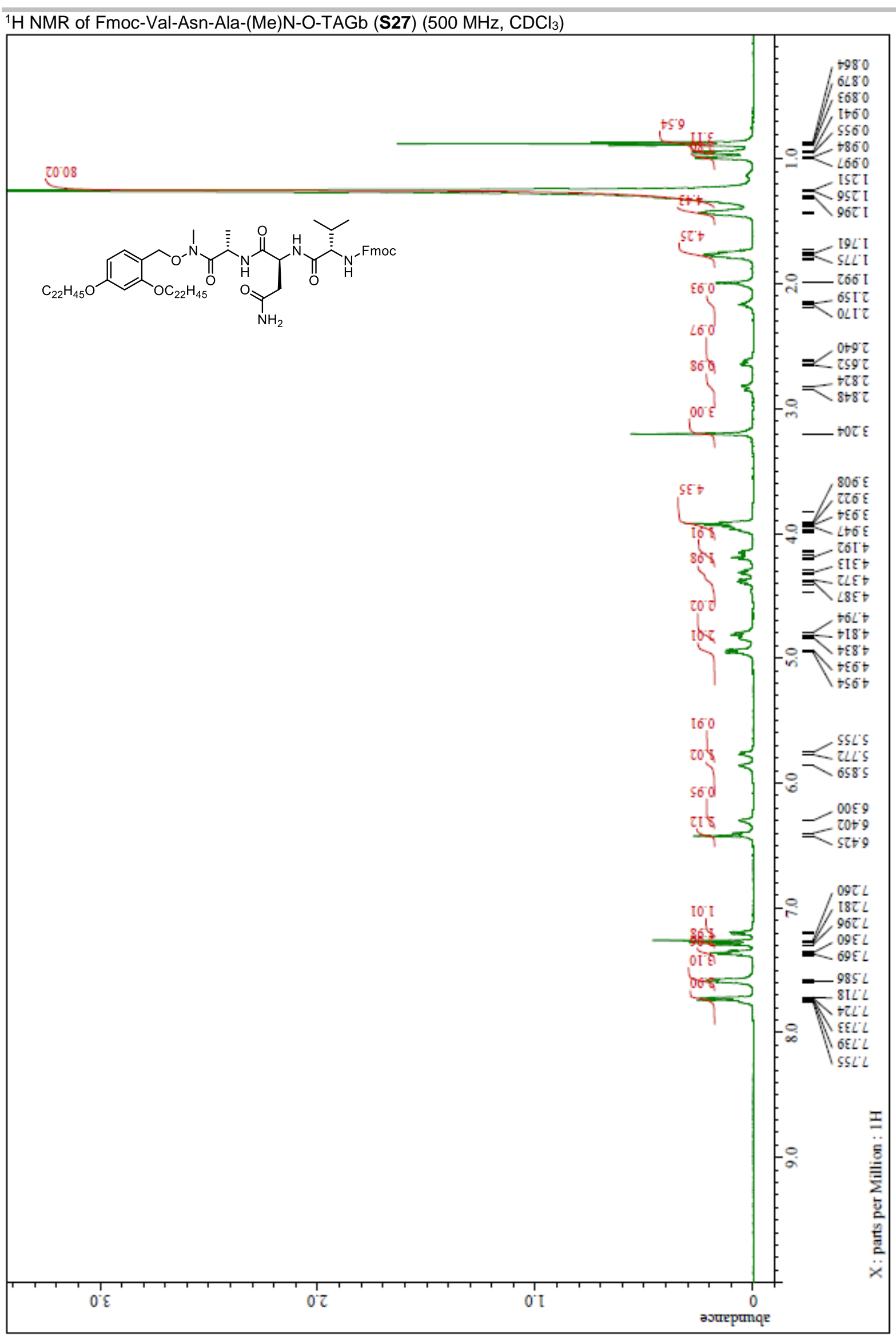




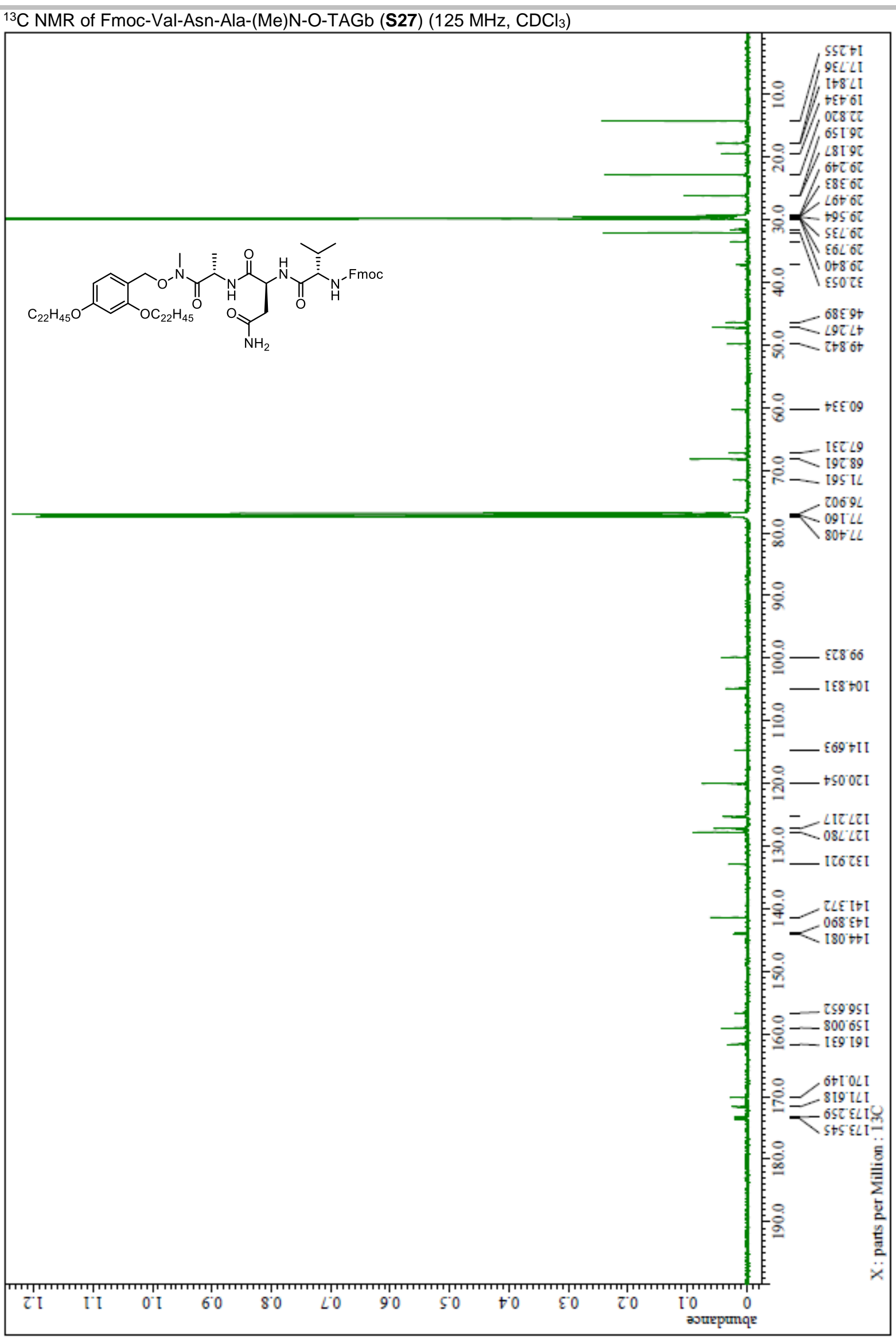




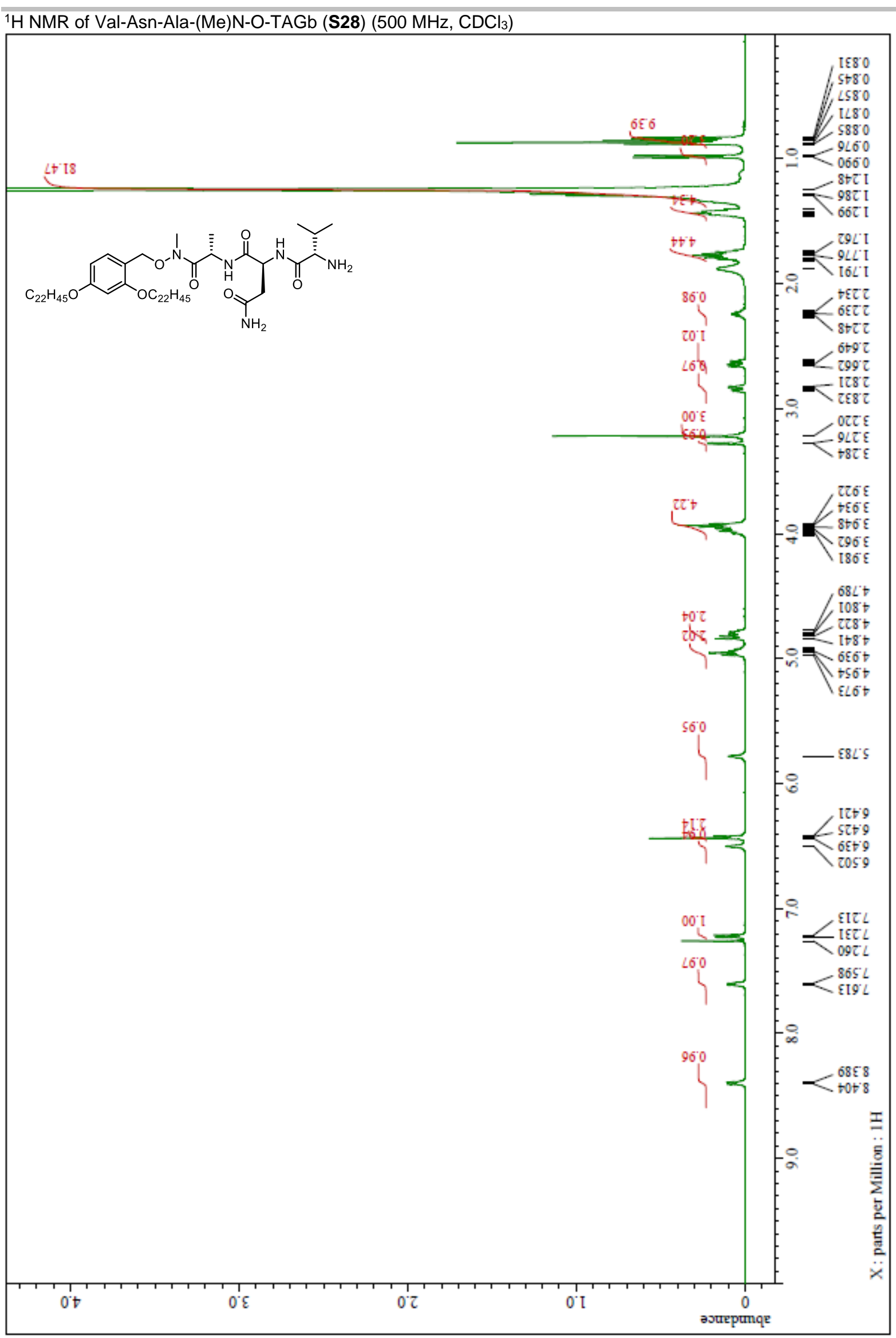




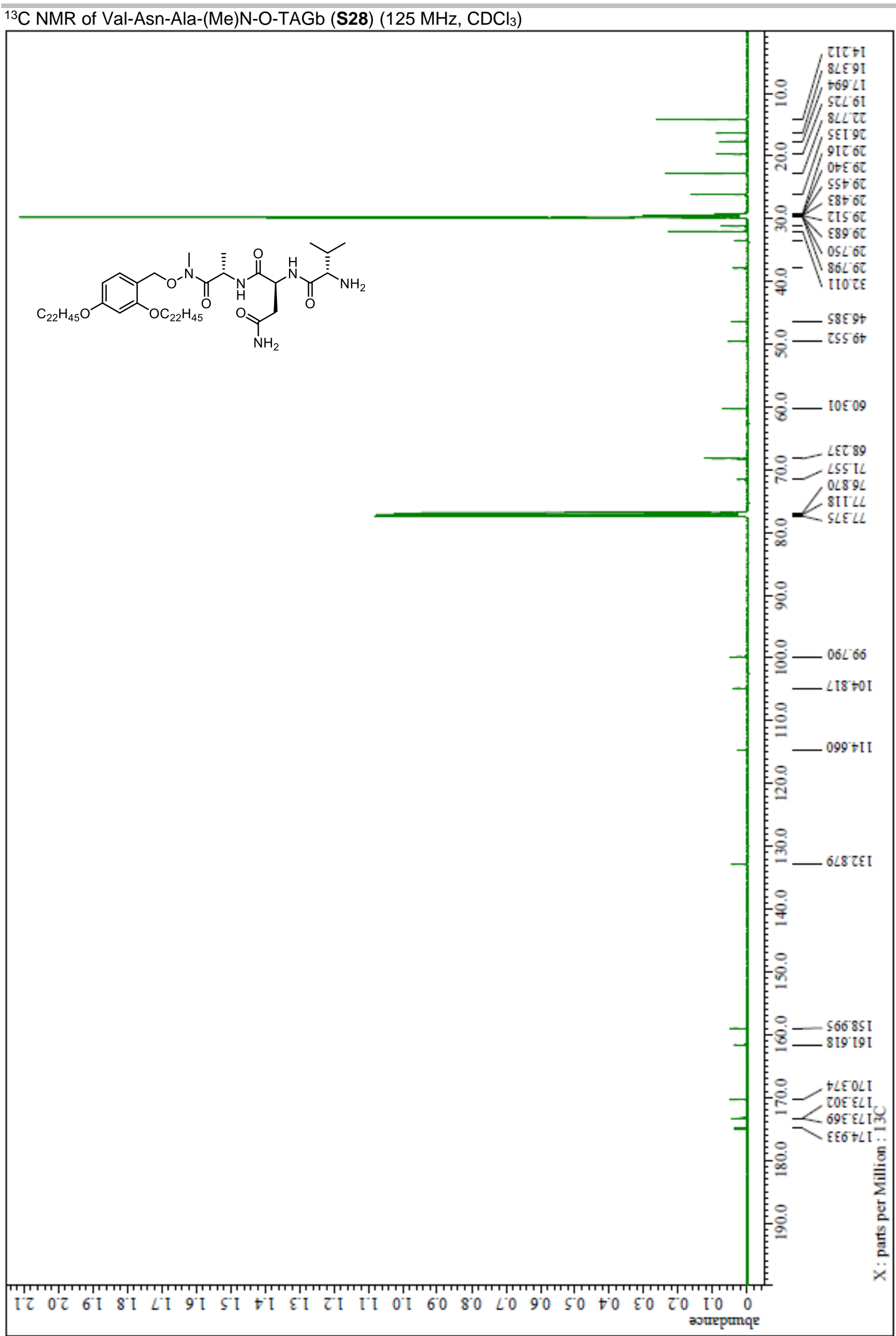




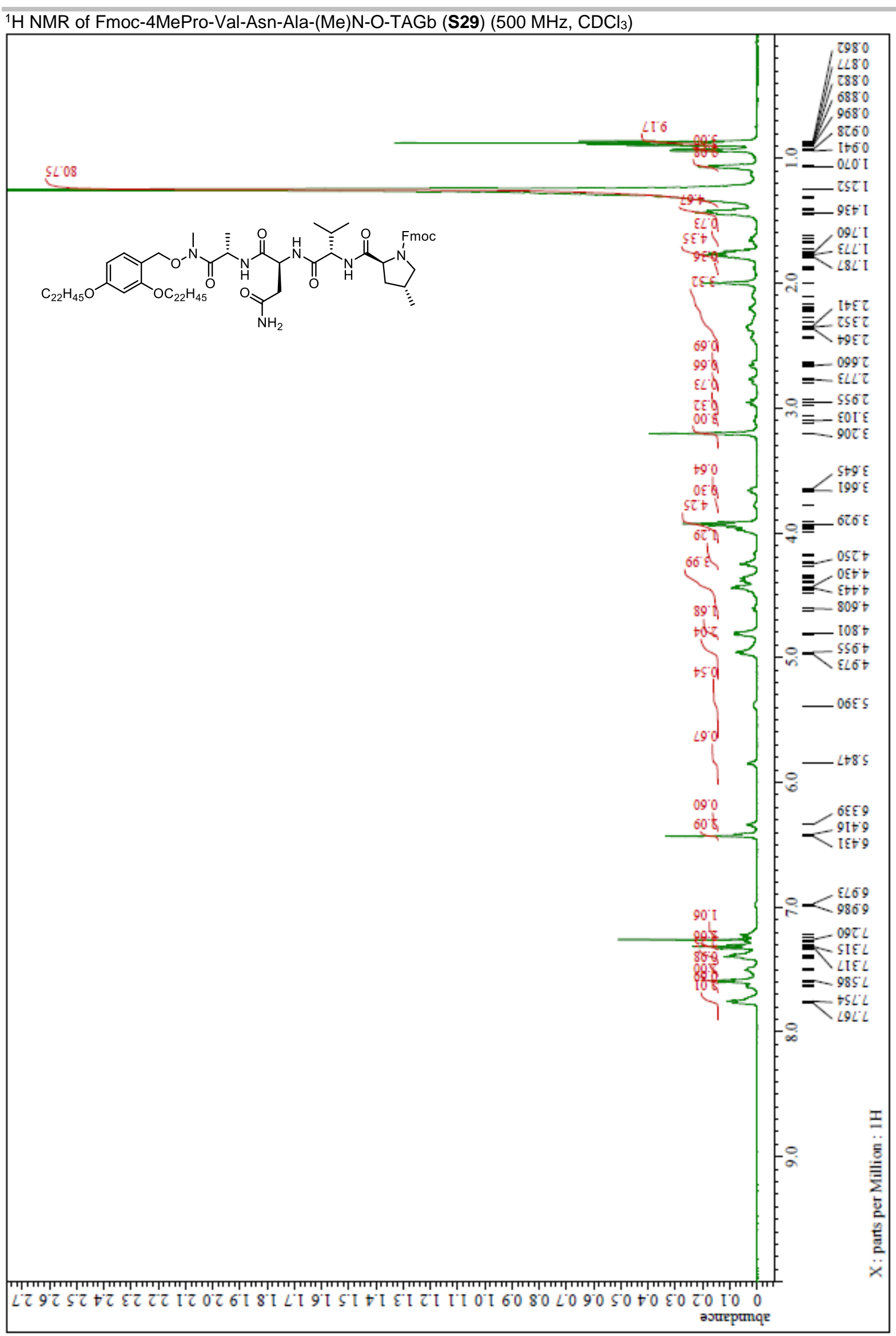




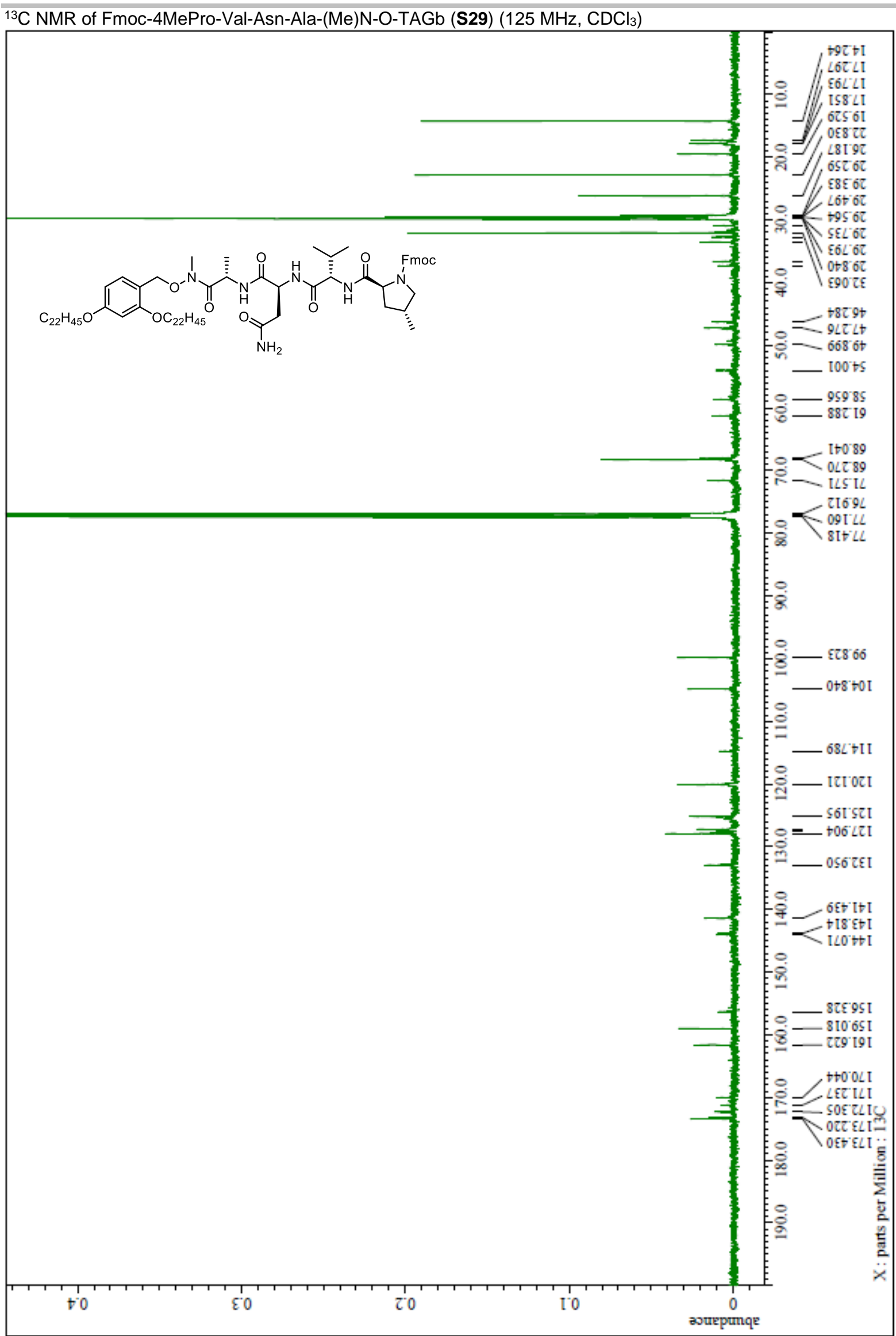




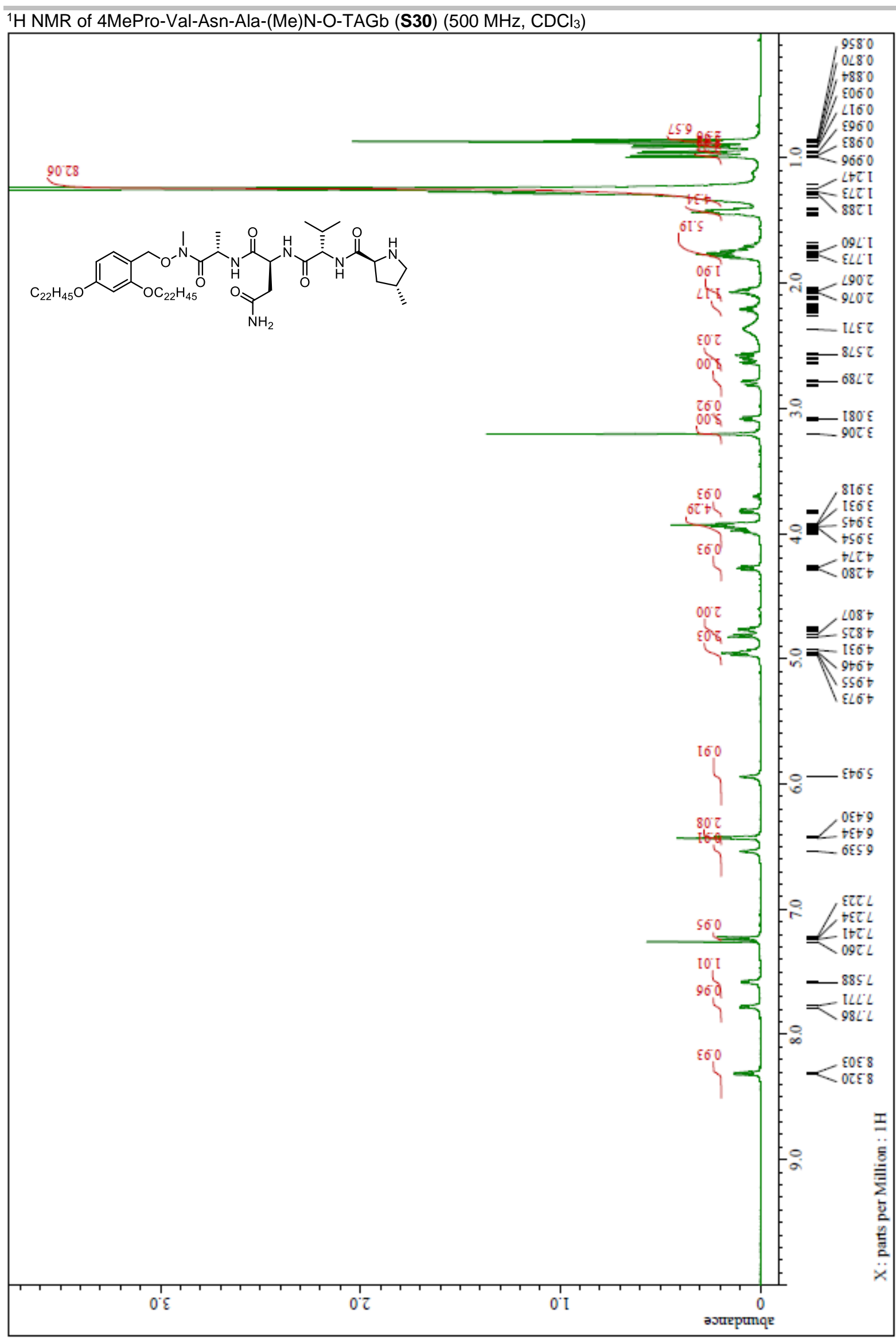




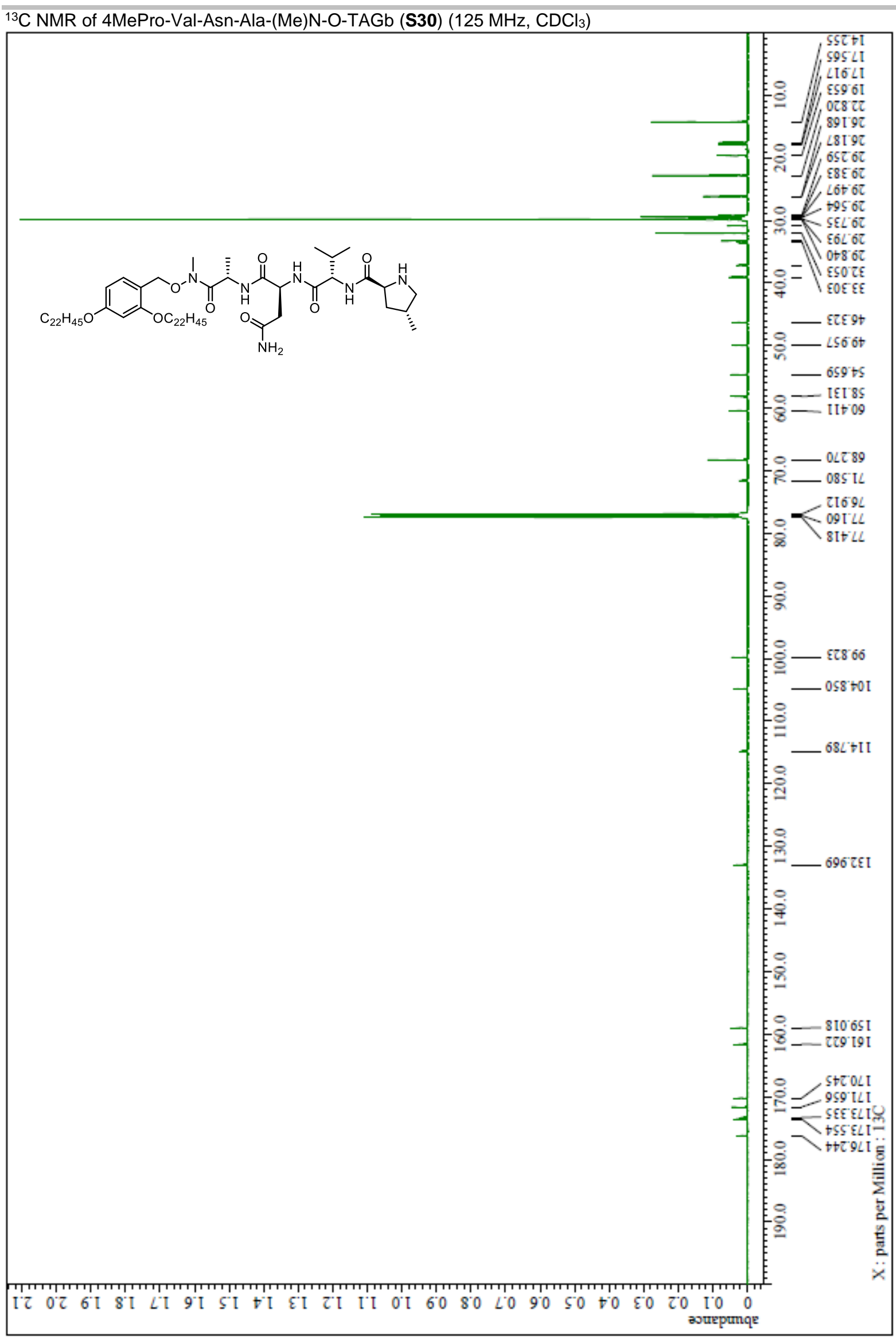




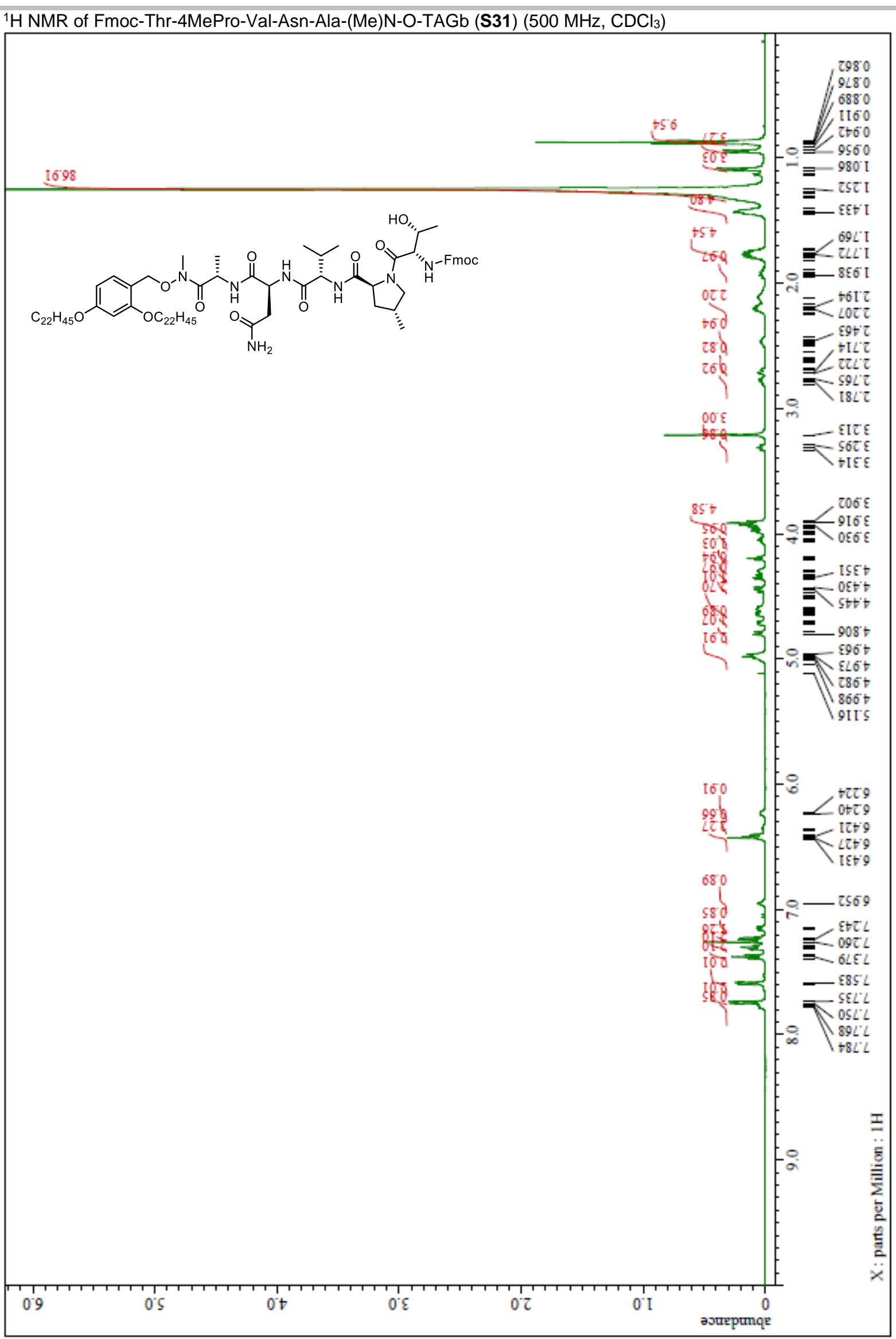




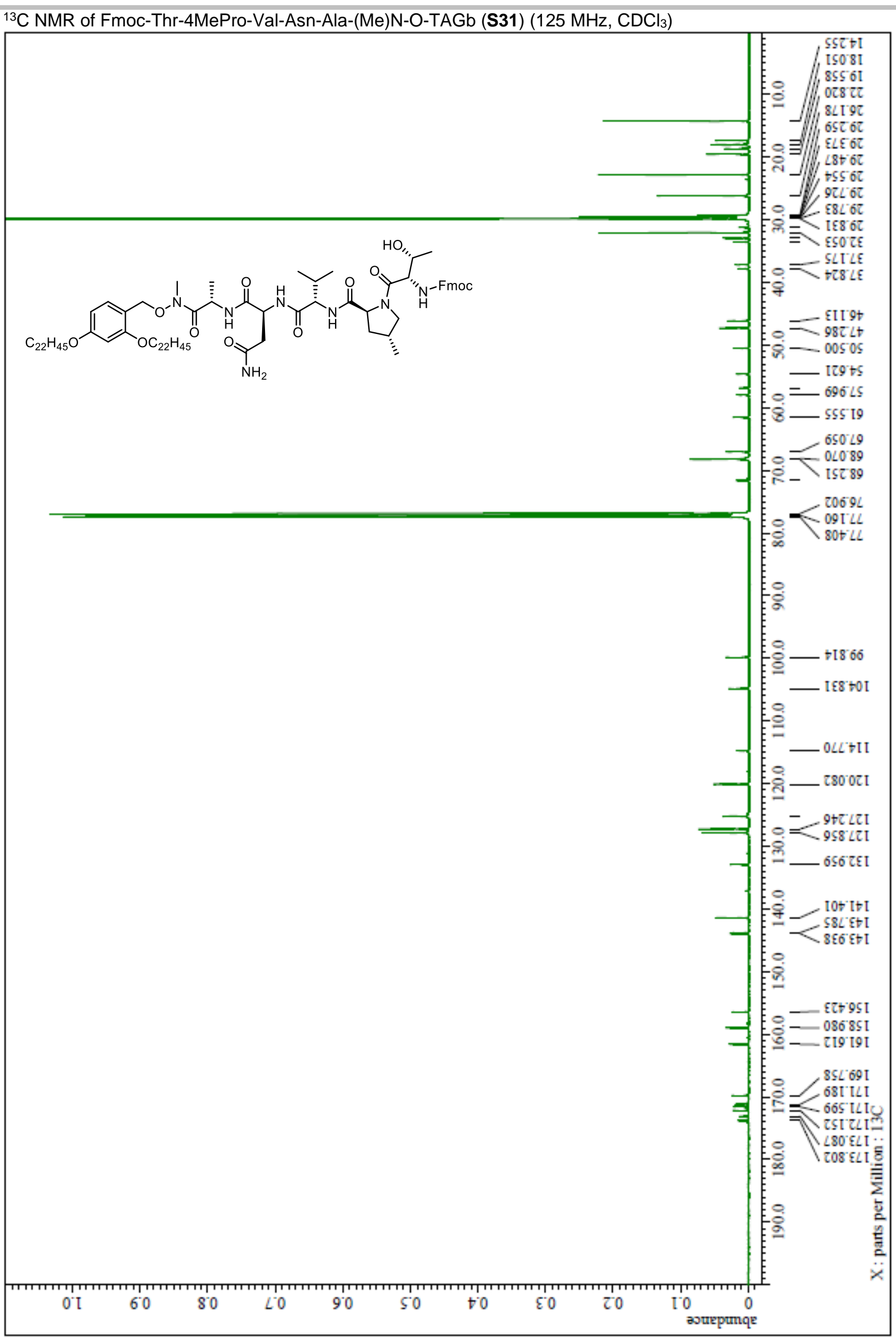




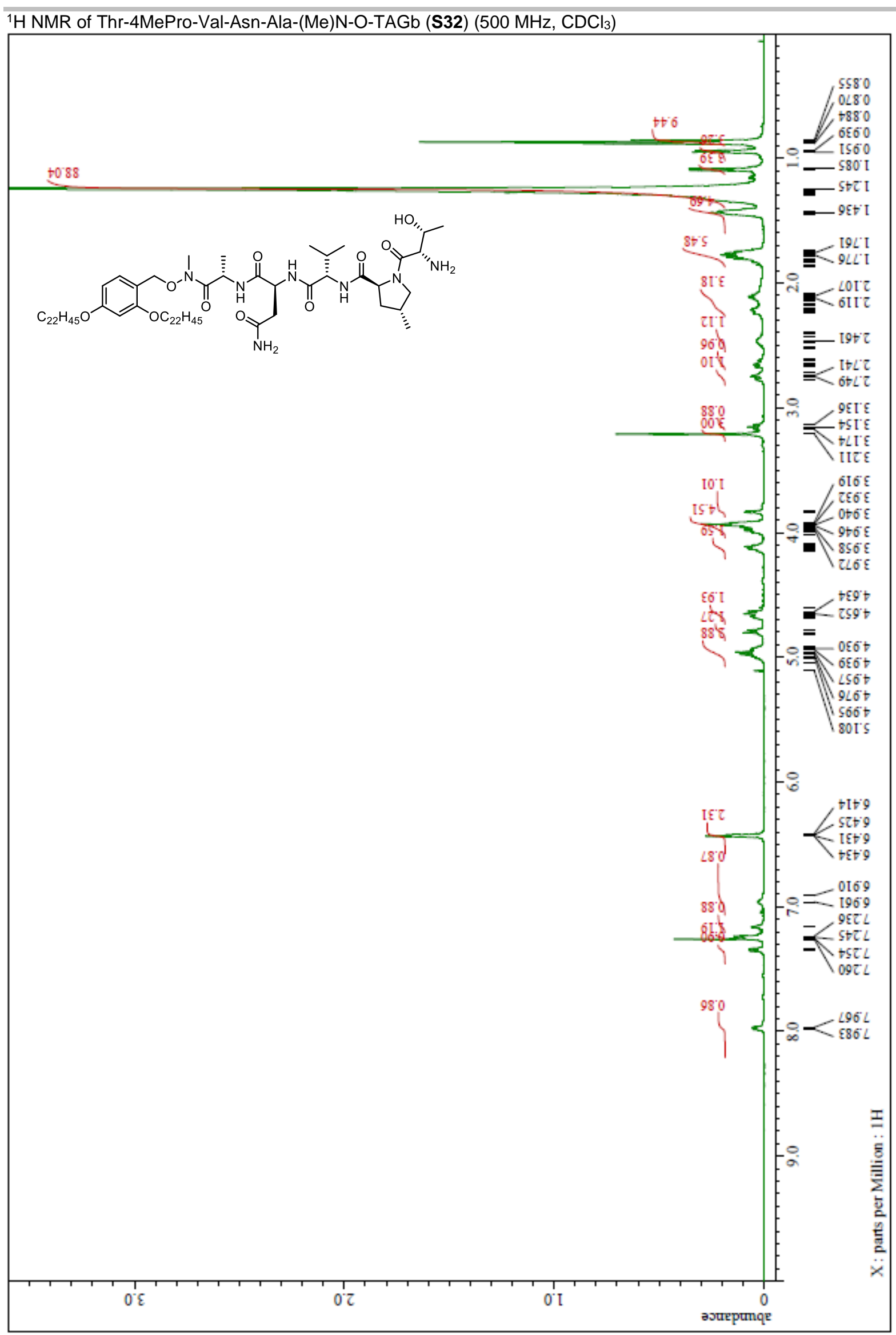




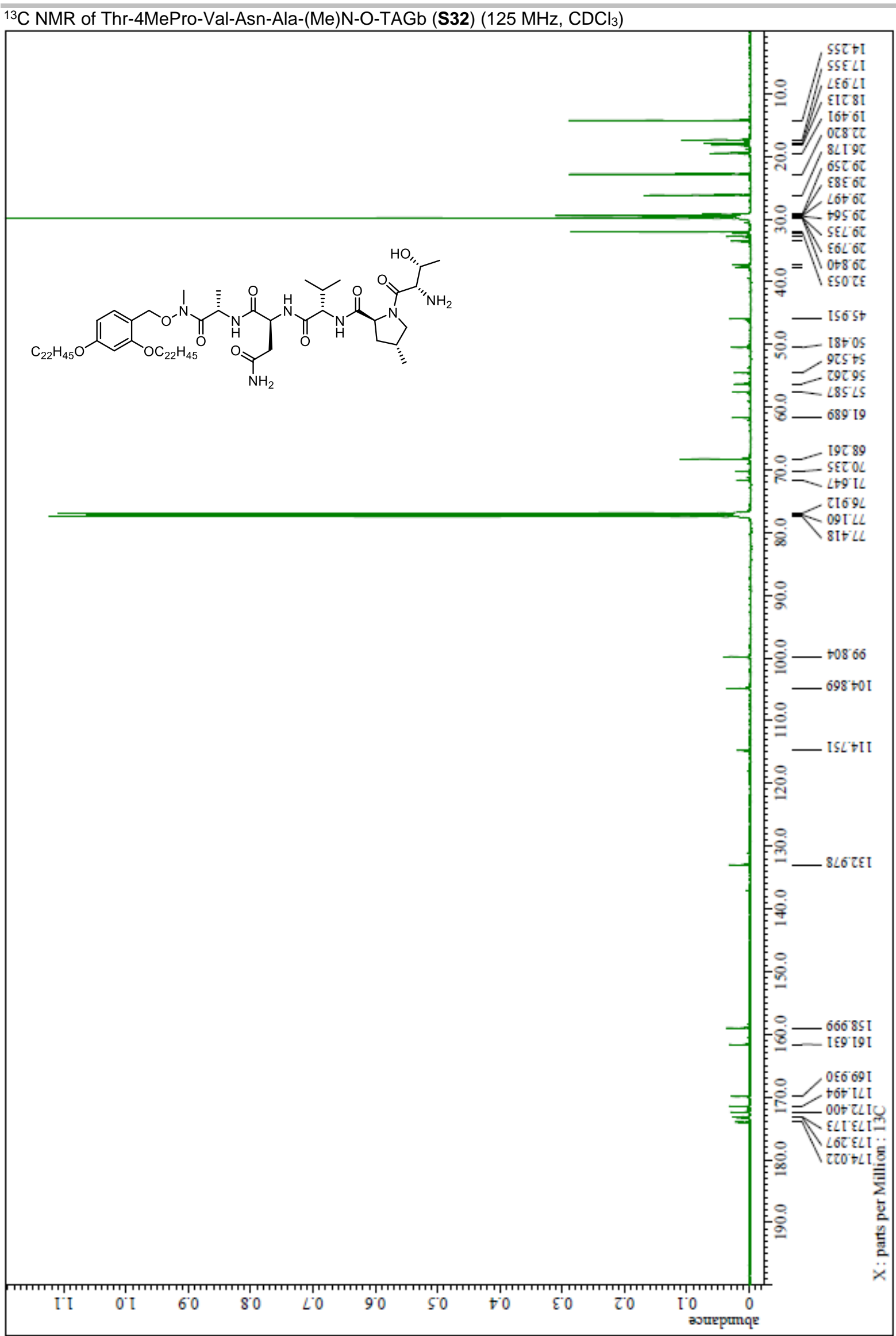




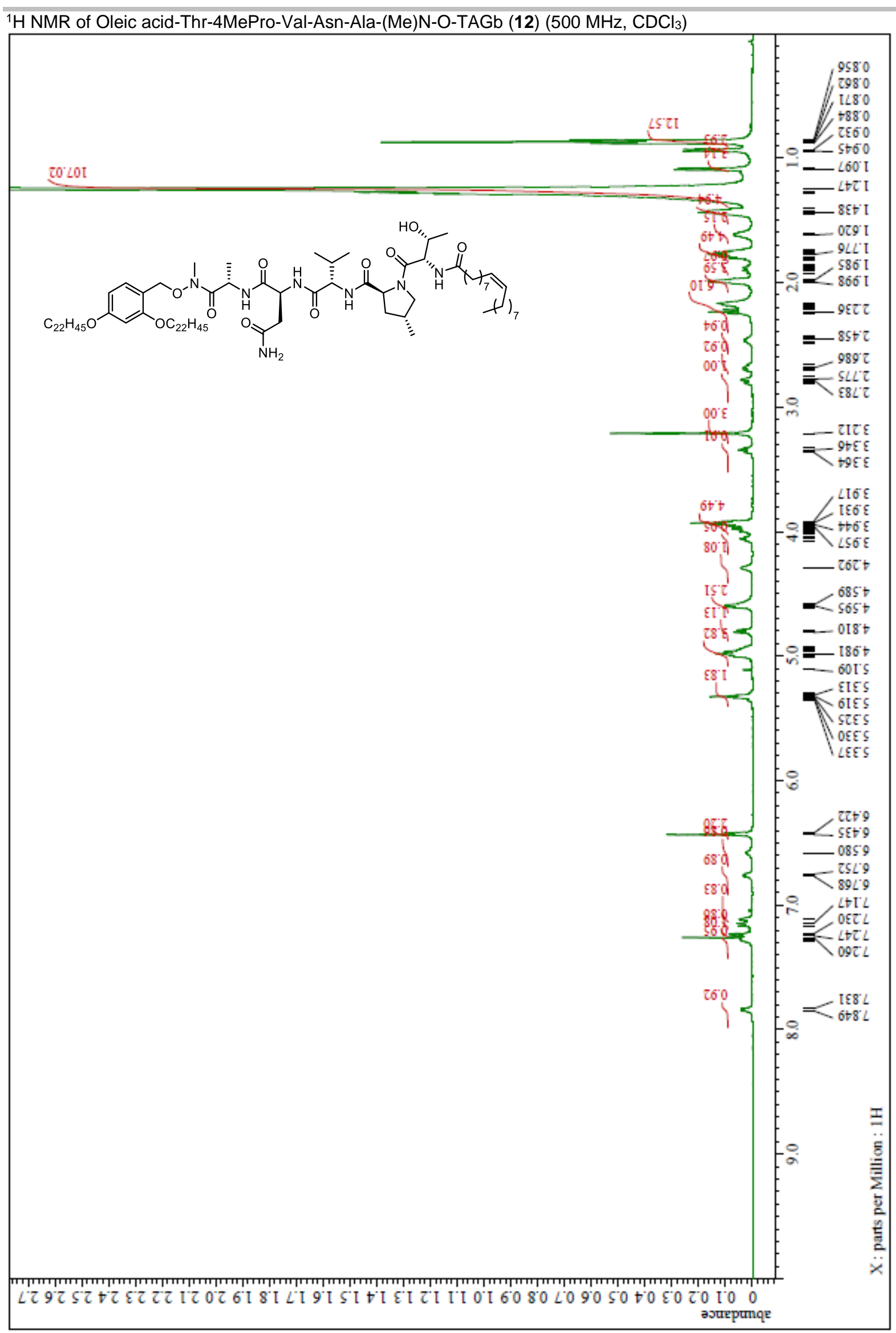




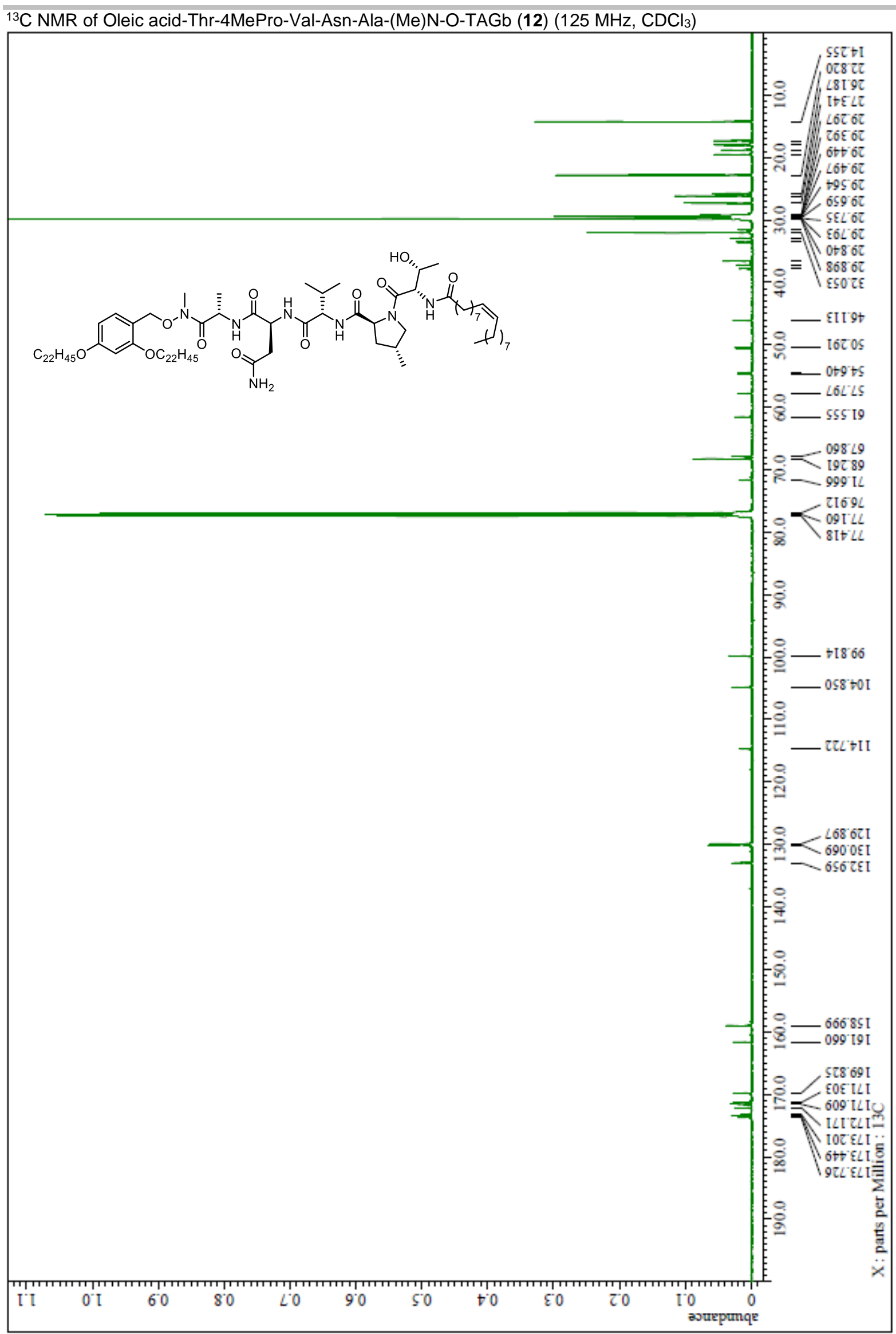




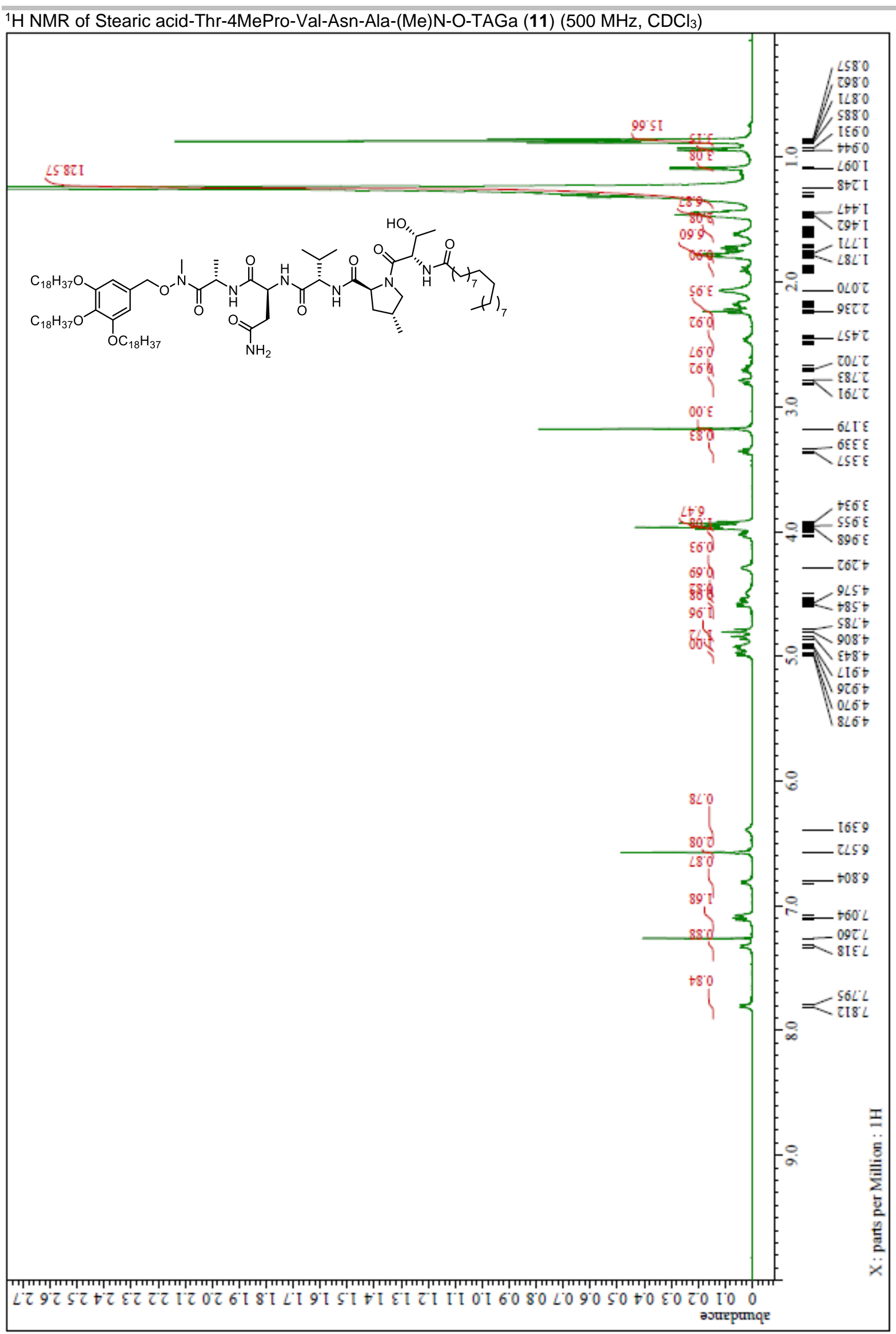




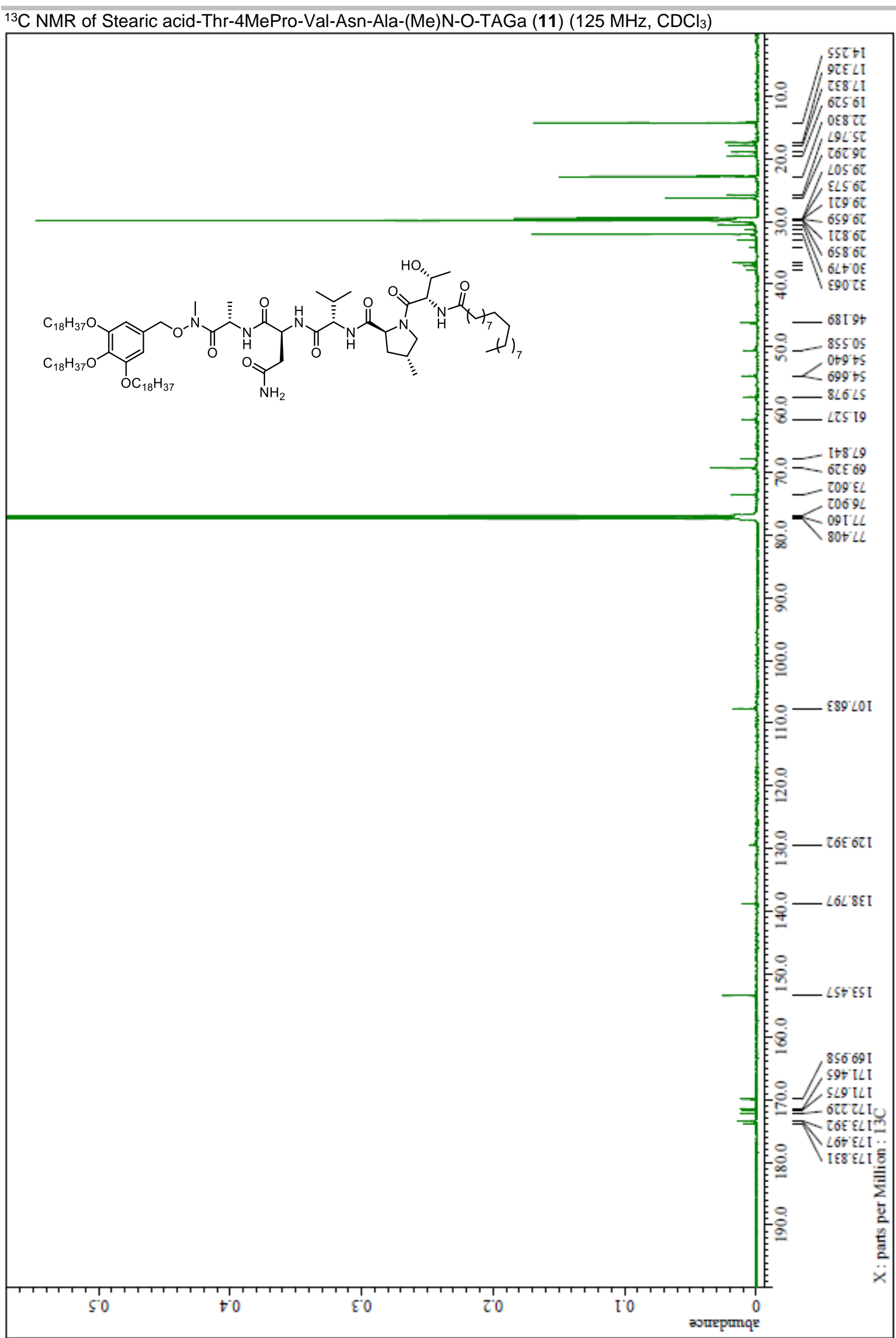




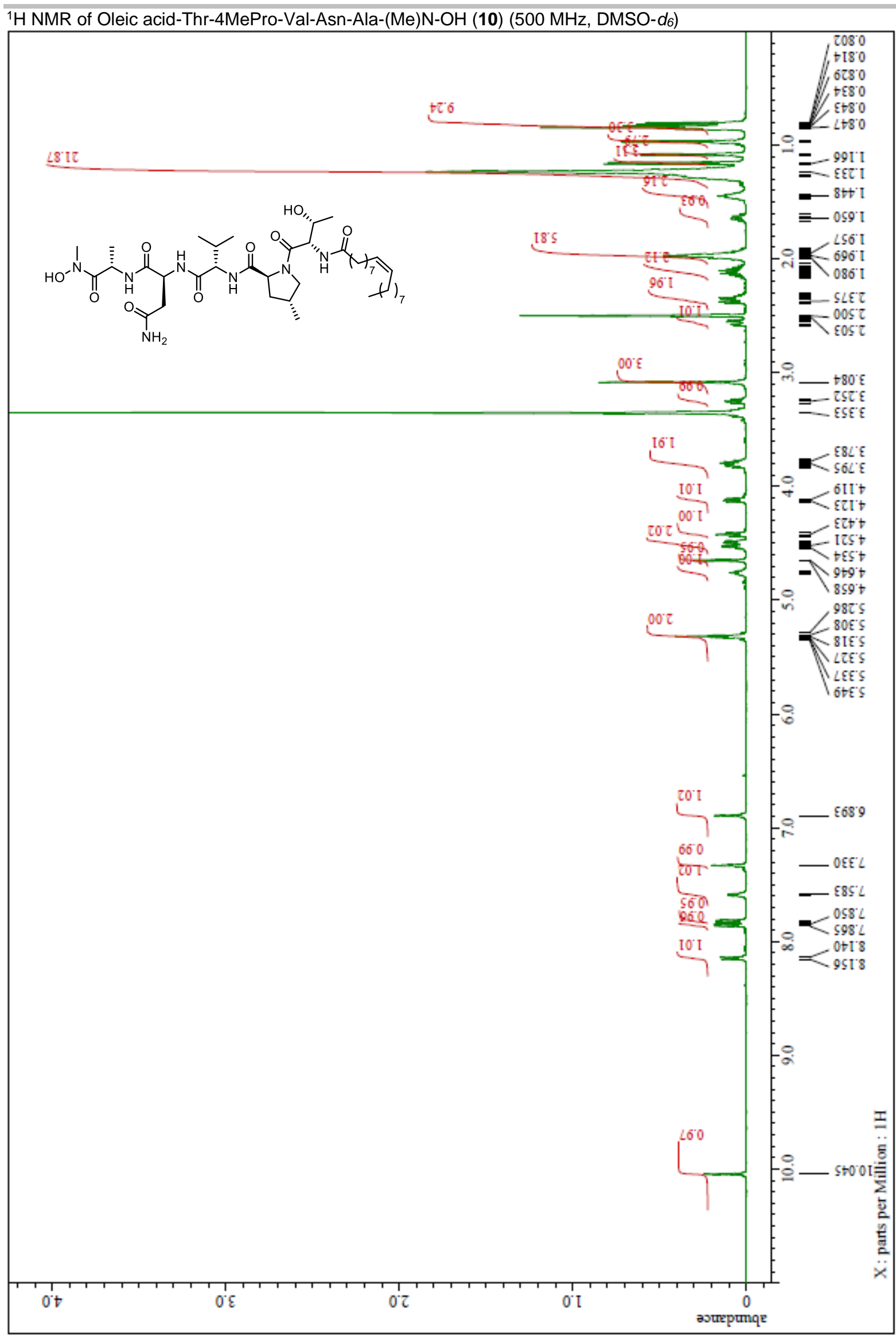




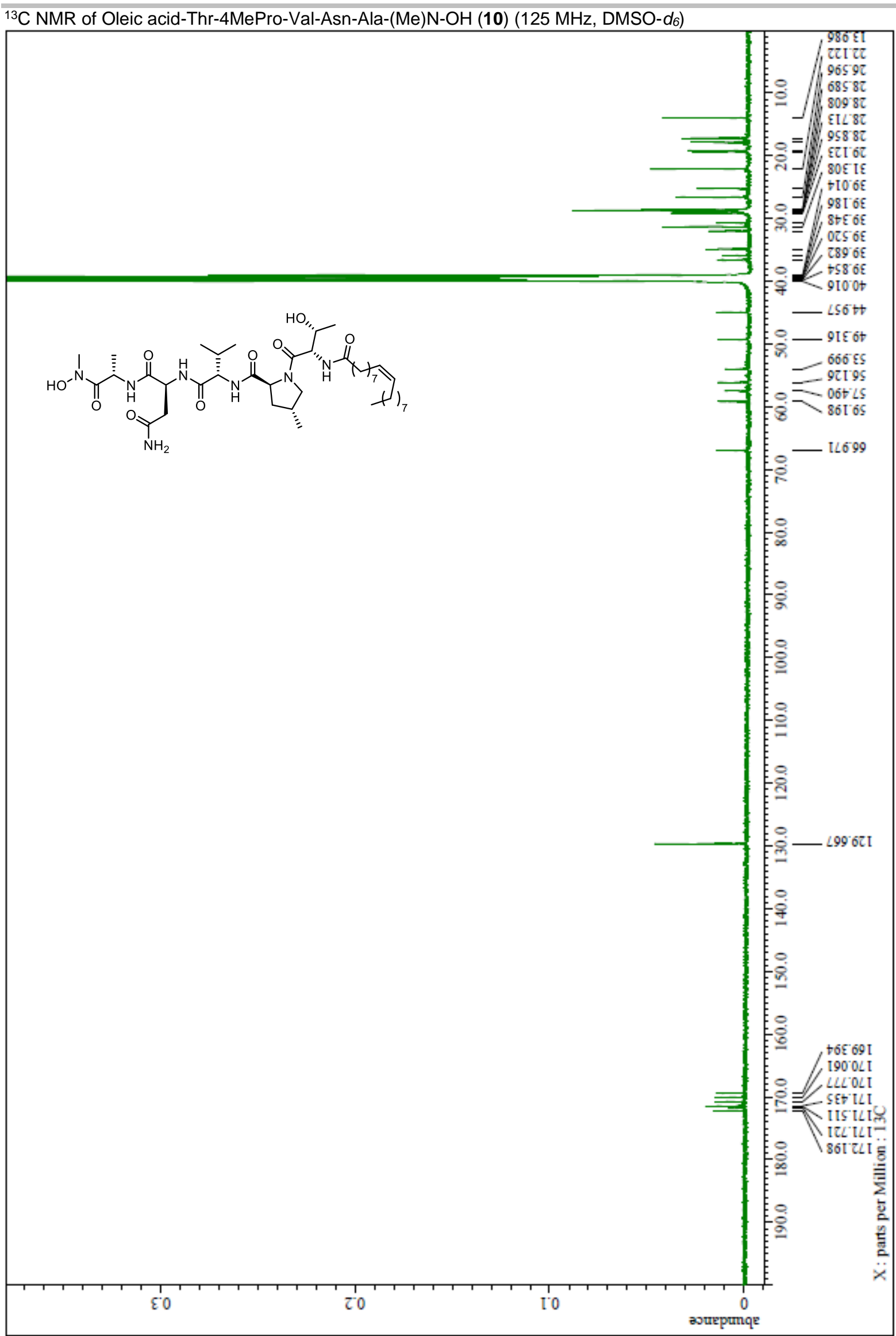




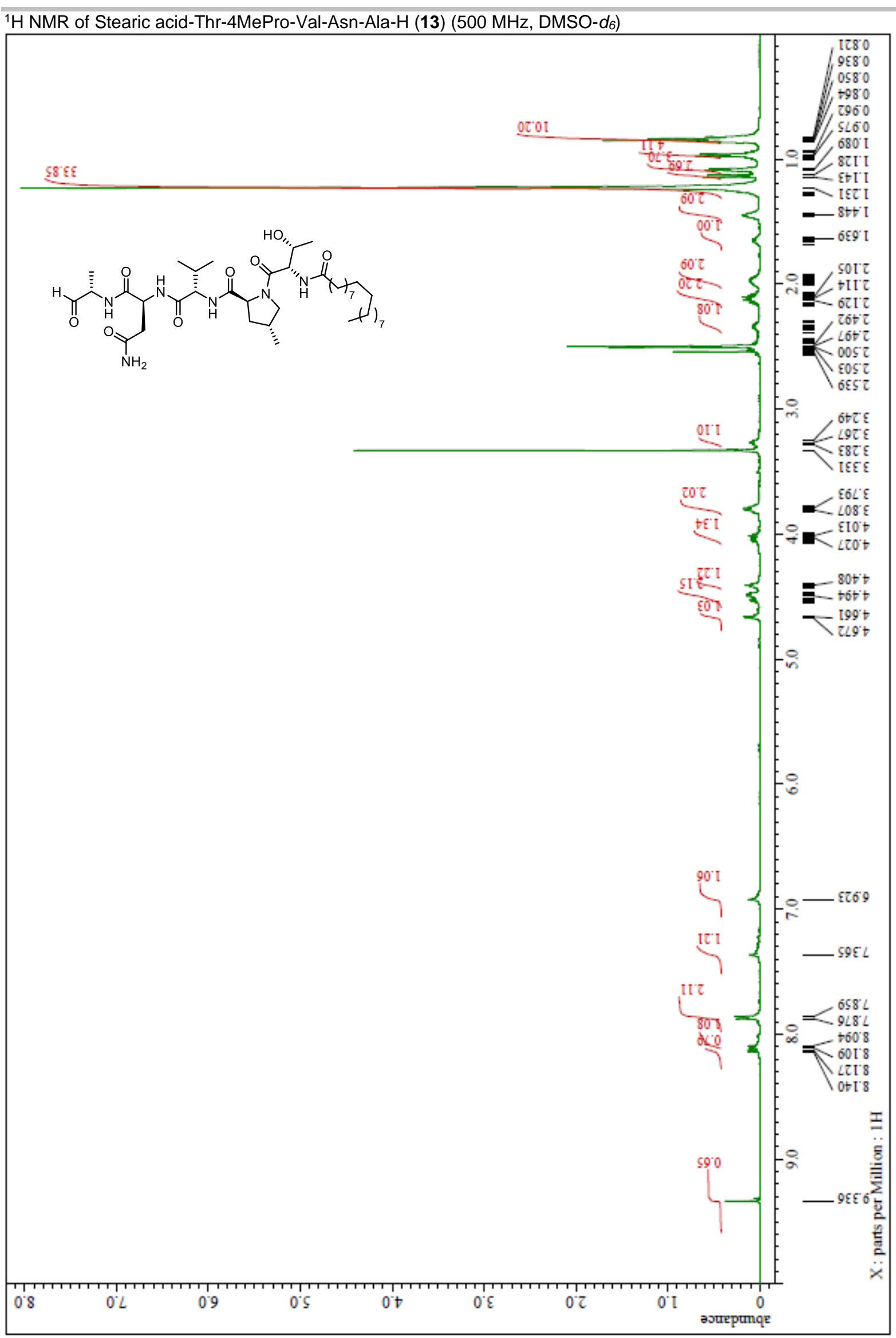




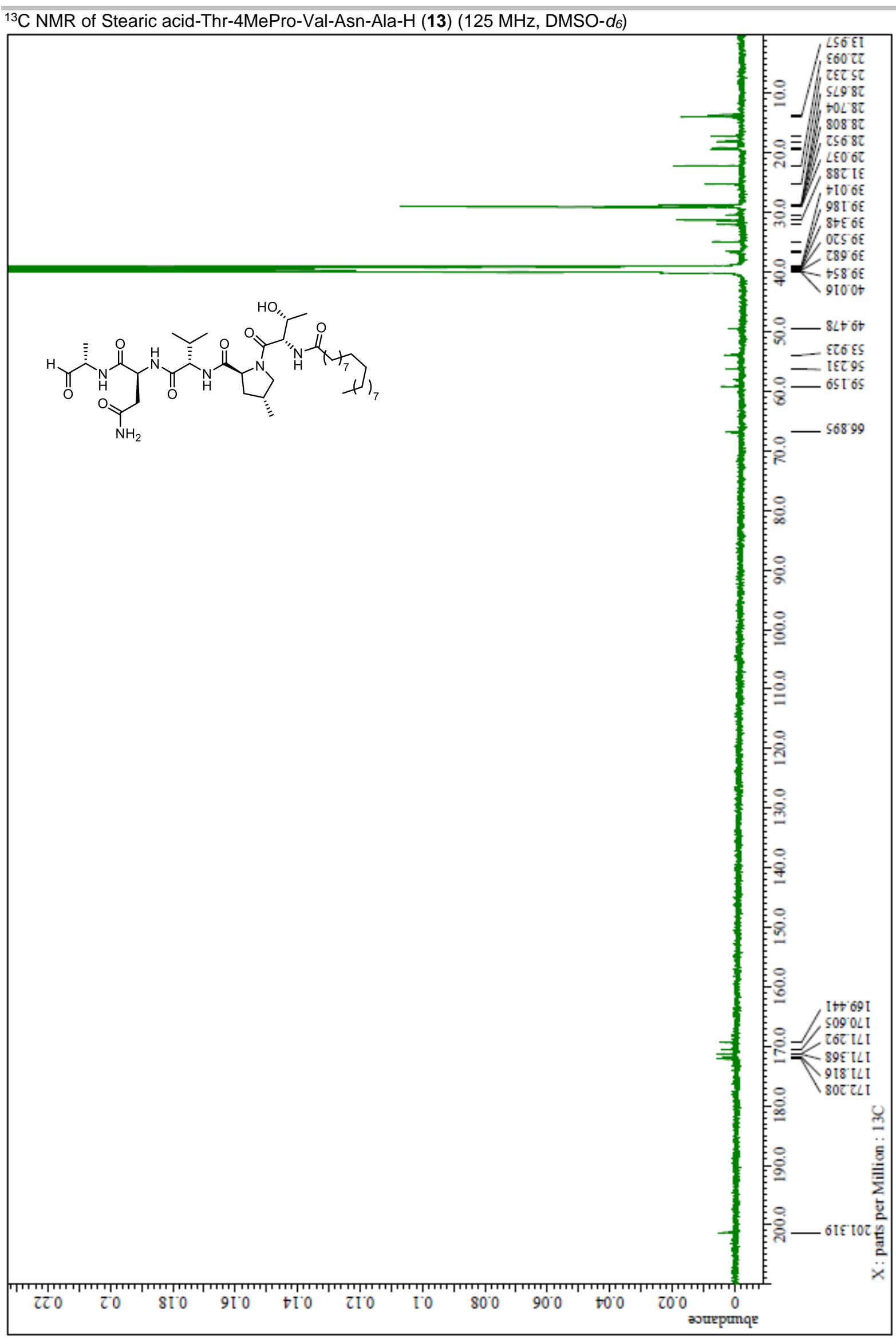

